\title{
Modeling human yolk sac hematopoiesis with pluripotent stem cells
}

by

Michael Harrison Atkins

A thesis submitted in conformity with the requirements

for the degree of Doctor of Philosophy

Medical Biophysics

University of Toronto

(C) Copyright by Michael H. Atkins 2020 


\title{
Modeling human yolk sac hematopoiesis with pluripotent stem cells
}

\author{
Michael H. Atkins \\ Doctor of Philosophy \\ Department of Medical Biophysics \\ University of Toronto
}

2020

\section{Abstract}

Embryonic hematopoiesis in the mouse consists of distinct programs that differ in their lineage potential and spatiotemporal organization. Prior to the generation of hematopoietic stem cells, the yolk sac initiates three programs, known as the primitive, erythro-myeloid progenitor (EMP) and lymphoid-primed multipotent progenitor (LMP) programs. While these programs do not contribute to long-term, multilineage hematopoiesis, their innate immune cell progeny seed and persist in the tissues throughout development and adult life. These populations execute organspecific functions to maintain homeostasis. Given the transient nature of their progenitors and their emergence at early stages of development, the identification and study of these programs has not been possible in the human embryo. To overcome this hurdle and access these progenitor populations, I established a developmental biology-guided protocol to generate the yolk sac hematopoietic programs from human pluripotent stem cells (hPSCs) in vitro. Using this strategy, I show that the human primitive hematopoietic program transitions through a NOTCH-dependent progenitor, known as the hemogenic endothelial cell (HEC), prior to the generation of the first hematopoietic cells. Coincident with the emergence of primitive hematopoietic cells, I demonstrate that a second population of HECs gives rise to the progenitors of the EMP program. This second 
HEC population also harbours $\mathrm{T}$ lymphoid potential indicative of the development of the LMP program. Clonal analyses revealed that the EMP and LMP programs derive from a common hematopoietic progenitor, defining in the human, a unified yolk sac program that develops from a multipotent cell. Together, the findings from my $\mathrm{PhD}$ project outline the architecture of the equivalent of the developing human yolk sac hematopoietic system and in doing so, offers a progenitor source for the study of the function of tissue-resident immune cells and their role in disease. 


\section{Acknowledgments}

I would like to thank my supervisor and mentor, Dr. Gordon Keller for his continued support throughout my scientific training. I am very appreciative of the time that he has invested in me, from our initial discussion of my research as a summer student and his research program at the Till and McCulloch Meeting in 2013 to our regular discussions during my time as a graduate student. Gordon has helped teach me how to think critically and perform rigorous science. The skills that I have developed in his lab will continue to be valuable, as I progress through the remaining stages of my training and in my career.

I would also like to thank Dr. Norman Iscove for his guidance. Norman's insightful questions led to productive discussions that benefited my work and growth as a scientist.

I have thoroughly enjoyed my time in Gordon's lab and I attribute a large part of that to my colleagues. I extend my thanks to present members of the lab: Marion Kennedy, Kelvin Chan Tung, Mark Gagliardi, Jamie Kwan, Brenda Cohen, Blair Gage, Xiaoning Sun, Vladimir Manchev, Alexander Mikryukov, Shunsuke Funakoshi, Nestor Fernandez, Donghe Yang, Ian Fernandes, Amine Mazine, Quynh Nguyen, Greg Kent and Mary White. I also thank former members of the lab: Analucia Garcia, Jee Hoon Lee, Adele Nikitina, Stephanie Protze, Shinichiro Ogawa, Mina Ogawa, Changi Cui and Marcela Hernandez.

I am fortunate to participate in research projects outside of the Keller lab through my work with Adriana Migliorini, Jelena Tomic and Simon Pascual-Gil.

I'm also appreciative of the past and present staff at the Sick Kids/UHN Flow Cytometry Facility for their assistance with cell sorting.

I am grateful for the support that I have received over the course of my training from the $\mathrm{MD} / \mathrm{PhD}$ program administration and my colleagues. I also acknowledge the sources of funding that have supported my training, including a Frederick Banting and Charles Best Canada Graduate Scholarship award.

I want to thank the scientists that first accepted me into their labs as an undergraduate student: Dr. Valerie Wallace and Dr. Jeff Smith. The opportunities that were afforded to me in their labs helped foster my interest in science. 
I am eternally grateful to the support that I have received from my parents, Harry Atkins and Suzanne MacMillan and my sister, Kathleen Atkins. They have always encouraged me to pursue my interests.

Finally, I want to thank my wife, Kelsey Atkins, who has been incredibly supportive throughout my training. Although the time commitments of grad school can be long and unpredictable, she always offered to help. I always look forward to seeing her and our cat, Kiton, at the end of the day. 


\section{Table of Contents}

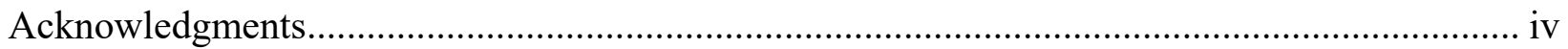

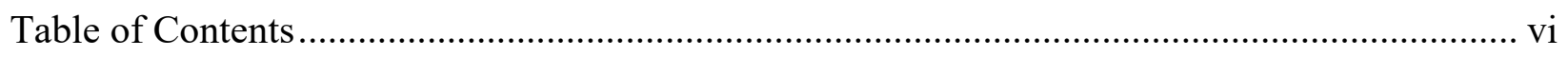

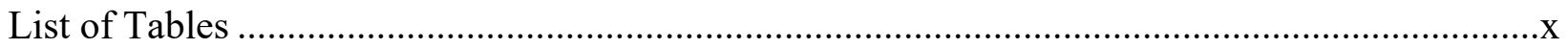

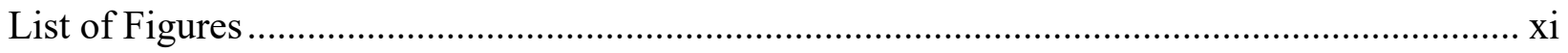

List of Abbreviations .................................................................................................... xiv

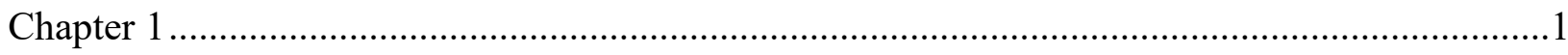

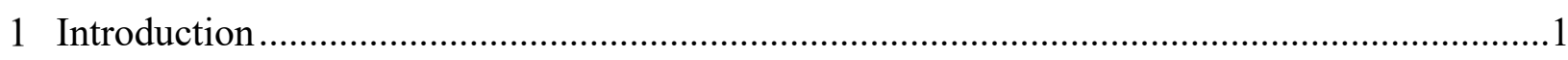

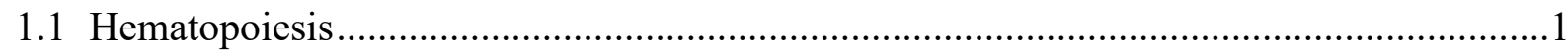

1.2 The embryonic hematopoietic programs ..................................................................

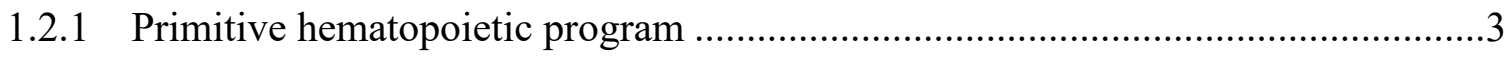

1.2.2 Erythro-myeloid progenitor hematopoietic program ...........................................

1.2.3 Lymphoid-primed multipotent progenitor hematopoietic program ......................6

1.2.4 Definitive hematopoietic program ............................................................

1.2.5 Contribution to the adult hematopoietic system ........................................ 11

1.3 Progenitors of embryonic hematopoietic programs .............................................. 15

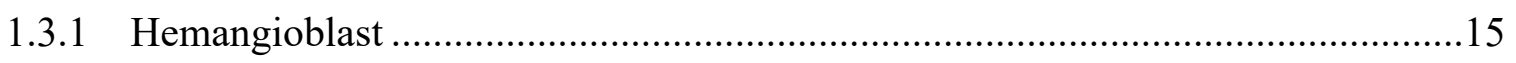

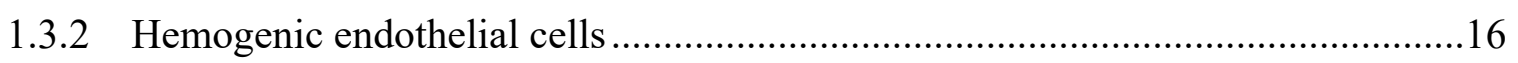

1.3.3 Relationship between the embryonic hematopoietic progenitors ........................18

1.4 Regulation of embryonic hematopoietic development ..........................................21

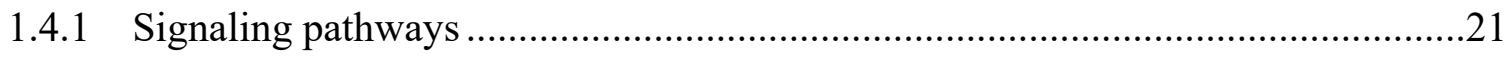

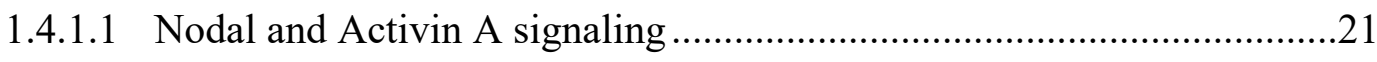

1.4.1.2 Bone morphogenic protein signaling...............................................22

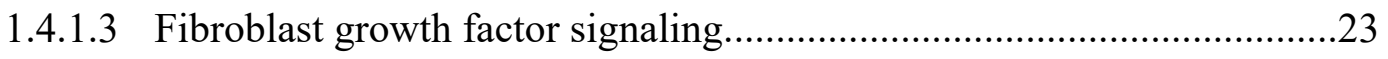

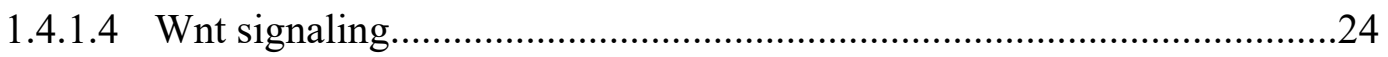

1.4.1.5 Vascular endothelial growth factor signaling ..................................25 
1.4.1.6 Notch signaling...................................................................................26

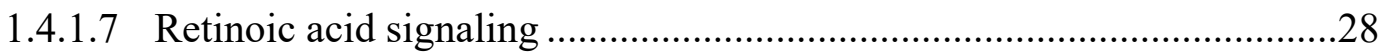

1.4.2 Transcription factor regulation of embryonic hematopoiesis ...............................29

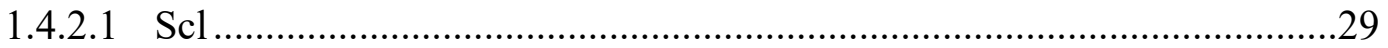

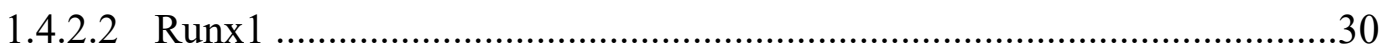

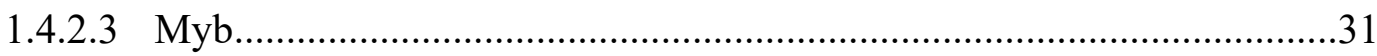

1.5 Modeling human embryonic hematopoietic development.................................................32

1.5.1 Hematopoietic development using human pluripotent stem cells ........................32

1.6 Applications of human pluripotent stem cell-derived hematopoietic cells.........................37

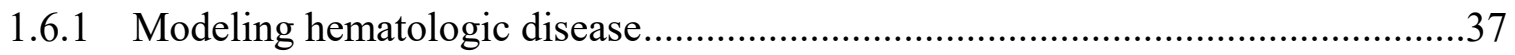

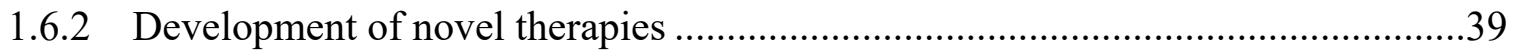

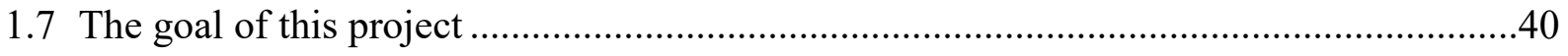

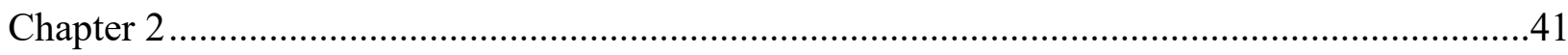

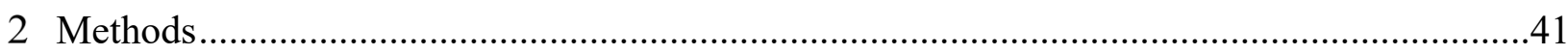

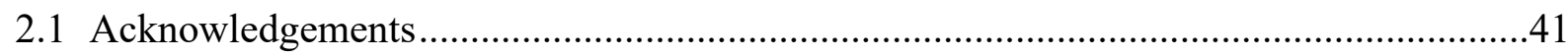

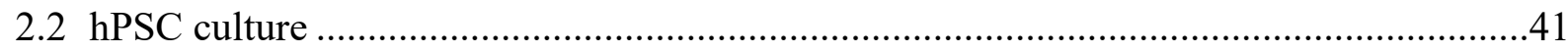

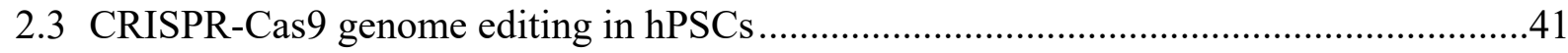

2.4 Hematopoietic differentiation from hPSCs....................................................................43

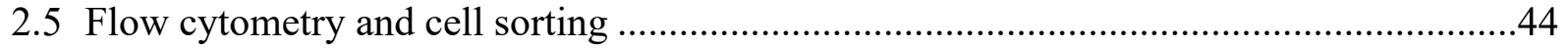

2.6 Quantitative real-time PCR ............................................................................................46

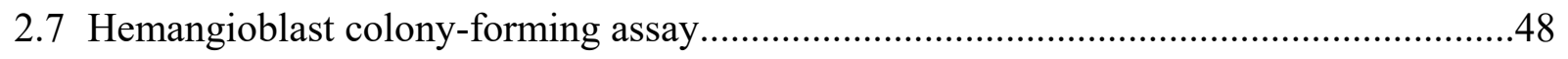

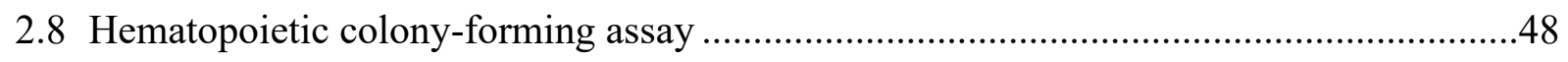

2.9 OP9-DL4 co-culture for T lymphoid differentiation ........................................................4

2.10HUVEC-E4ORF1 co-culture for hematopoietic cell expansion.........................................48

2.11 Hematopoietic multipotent progenitor cell assay .............................................................49

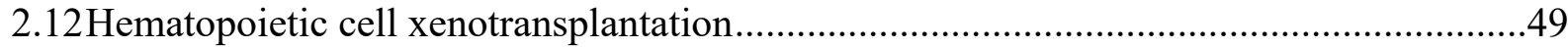


2.13Lentiviral transduction of hPSC-derived cells .............................................................50

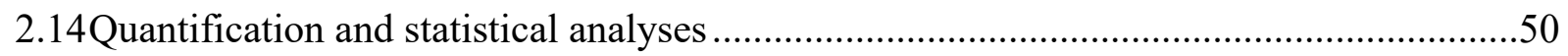

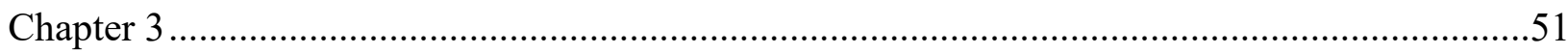

3 Architecture of human yolk sac hematopoietic development using hPSCs...............................51

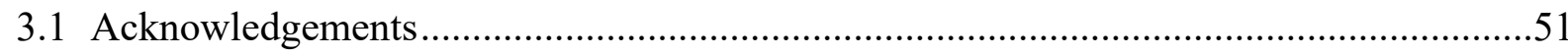

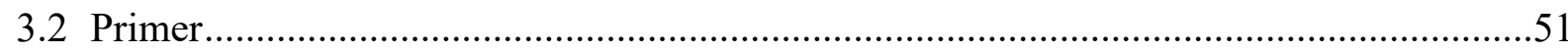

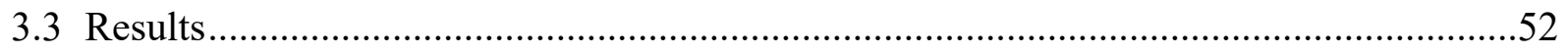

3.3.1 Specification of the primitive hematopoietic program .......................................52

3.3.2 The primitive hematopoietic program transitions through a hemogenic endothelial cell intermediate ...............................................................................59

3.3.3 The primitive hematopoietic program is dependent on NOTCH signaling ...........62

3.3.4 The primitive hematopoietic program requires MYB ……………………….....65

3.3.5 Identification of a second hemogenic endothelial cell population with broader hematopoietic lineage potential ..........................................................................71

3.3.6 The erythro-myeloid and T lymphoid progenitors are generated from multipotent hematopoietic progenitors ……………….......................................8

3.3.7 Engraftment potential of multipotent hematopoietic progenitors..........................88

3.3.8 Aldehyde dehydrogenase activity during hematopoietic development ..................88

3.3.9 Hematopoietic development from human pluripotent stem cell lines ....................91

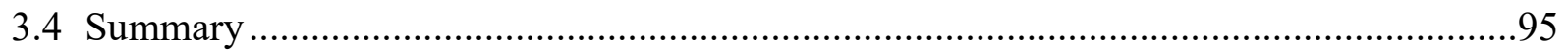

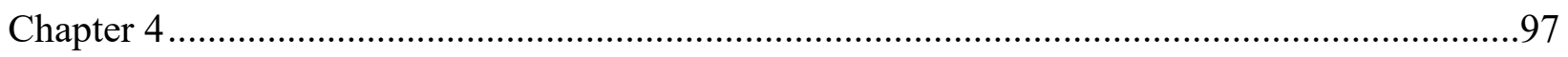

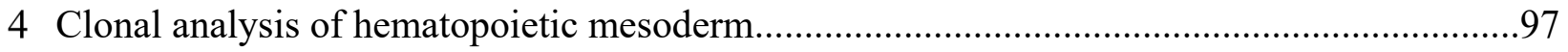

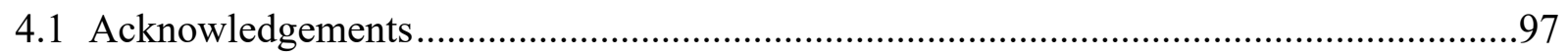

4.2 Primer

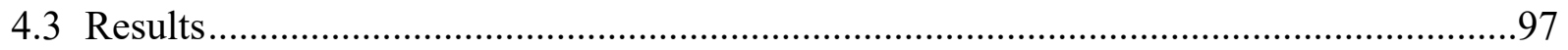

4.3.1 Development and potential of BL-CFC-derived blast colonies.............................97

4.3.2 Clonal analysis hematopoietic mesoderm.........................................................104 


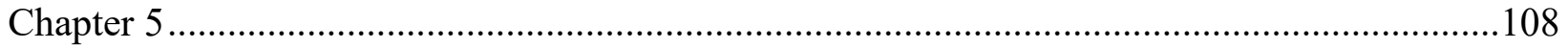

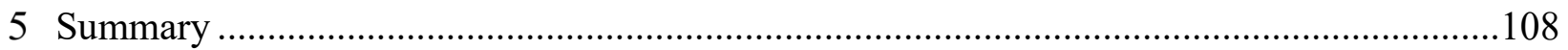

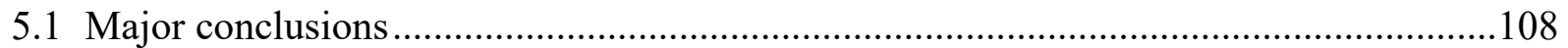

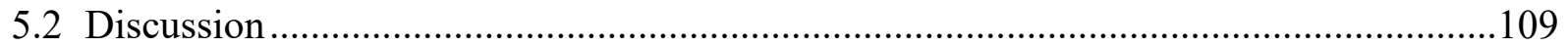

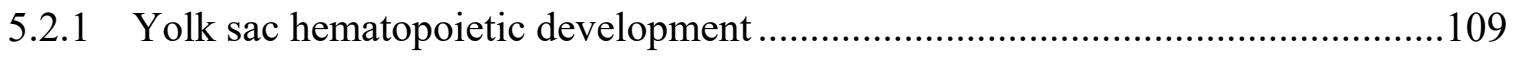

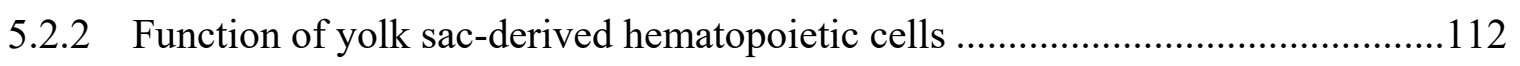

5.2.3 Regulation of yolk sac hematopoietic development .....................................115

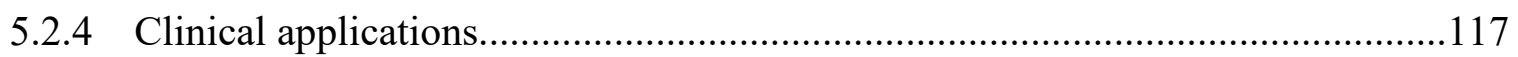

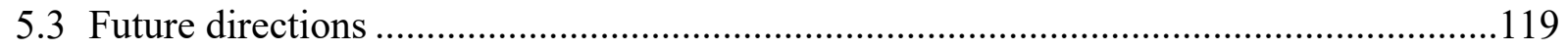

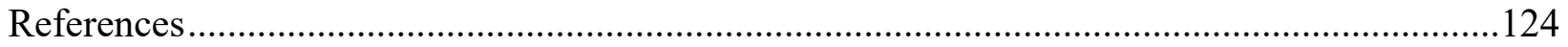




\section{List of Tables}

Table 2.1 Sequence information for CRISPR-Cas9 genome editing...................................... 42

Table 2.2 Primer sequences used to screen hESC clones.................................................... 43

Table 2.3 Primer sequences for RT-qPCR ............................................................. 47 


\section{List of Figures}

Figure 1.1 Mouse embryonic hematopoietic development. 14

Figure 1.2 The hemangioblast. 19

Figure 1.3 Hemogenic endothelial cells 20

Figure 1.4 Model of human hematopoietic development using pluripotent stem cells. 36

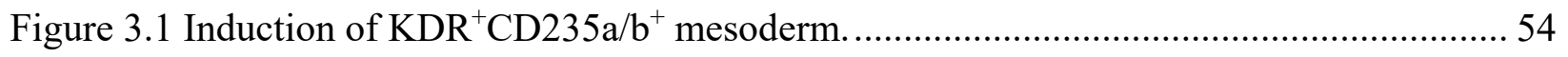

Figure 3.2 Emergence of the primitive hematopoietic program from hPSCs.......................... 57

Figure 3.3 Hematopoietic potential of $\mathrm{KDR}^{+}$mesoderm.................................................... 58

Figure 3.4 Characterization of $\mathrm{KDR}^{+} \mathrm{CD} 34^{+}$cells at day 5 of differentiation. .......................... 60

Figure 3.5 The primitive hematopoietic program transitions through a hemogenic endothelial cell intermediate. 61

Figure 3.6 NOTCH signaling during the development of the primitive hematopoietic program. 63

Figure 3.8 NOTCH signaling is required for the generation of the hematopoietic cells of the primitive hematopoietic program. 64

Figure 3.9 Generation and characterization of $\mathrm{MYB}^{-/-} \mathrm{H} 1 \mathrm{hESCs}$. 67

Figure $3.10 \mathrm{MYB}$ is not required for the generation of $\mathrm{KDR}^{+} \mathrm{CD} 235 \mathrm{a}^{+}$mesoderm. 68

Figure $3.11 \mathrm{MYB}$ is not required for the generation of $\mathrm{KDR}^{+} \mathrm{CD} 34^{+}$cells. 69

Figure 3.12 MYB is required for the generation of the hematopoietic cells of the primitive hematopoietic program. 70

Figure 3.13 Characterization of $\mathrm{CD} 34^{+} \mathrm{CD} 43^{-}$cells at day 6 of differentiation. 72

Figure 3.14 Separation of two hematopoietic programs at day 6 of differentiation based on CD34 and CD43 expression. 
Figure $3.15 \mathrm{CD}^{+} 4^{+} \mathrm{CD} 43^{-} \mathrm{HECs}$ give rise to $\mathrm{CD} 45^{+}$hematopoietic progenitors.

Figure 3.16 CD34 ${ }^{+} \mathrm{CD} 43^{-} \mathrm{HECs}$ have $\mathrm{T}$ lymphoid potential 79

Figure $3.17 \mathrm{KDR}^{+} \mathrm{CD} 235 \mathrm{a} / \mathrm{b}^{+}$mesoderm gives rise to the $\mathrm{T}$ lymphoid lineage. 80

Figure 3.18 Analyses of T lymphoid progenitors. 81

Figure 3.19 Characterization of the day $6 \mathrm{CD} 43^{+}$and $\mathrm{CD} 34^{+} \mathrm{CD} 43^{-} \mathrm{HEC}$-derived hematopoietic cells. 83

Figure 3.20 The expression of CD90 and absence of CD7 marks a CD34 ${ }^{+} \mathrm{CD} 45^{+}$hematopoietic progenitor population with multilineage potential

Figure 3.21 The $\mathrm{CD} 34^{+} \mathrm{CD} 45^{+} \mathrm{CD} 90^{+} \mathrm{CD}^{-}$population contains multipotent hematopoietic progenitors 87

Figure 3.22 Engraftment potential of hPSC-derived multipotent hematopoietic progenitors...... 89

Figure 3.23 The primitive and multipotent progenitor programs do not develop from $\mathrm{ALDH}^{+}$ progenitors. 90

Figure 3.24 Generation of the yolk sac hematopoietic lineage from CHOP10WT iPSCs. 93

Figure 3.25 Hematopoietic potential of $\mathrm{KDR}^{+} \mathrm{CD} 235 \mathrm{a} / \mathrm{b}^{+}$mesoderm generated from CHOP10WT iPSCs. 94

Figure 3.26 Model of human yolk sac hematopoietic development using pluripotent stem cells.96 Figure 4.1 BL-CFC-derived blast colony development 99

Figure 4.2 The addition of the TGF $\beta$ inhibitor, SB431542 enhances BL-CFC-derived blast colony development. 100

Figure 4.3 Hematopoietic potential of BL-CFC-derived cultures. 102

Figure 4.4 BL-CFC-derived blast colonies do not efficiently give rise to $\mathrm{CD} 34^{+} \mathrm{CD} 45^{+}$cells .. 103 
Figure 4.5 Lentivirus-based strategy to track the primitive erythroid and T lymphoid potential of

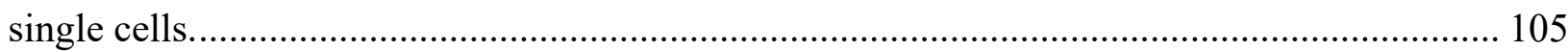

Figure 4.6 Lentiviral transduction of $\mathrm{KDR}^{+} \mathrm{CD} 235 \mathrm{a} / \mathrm{b}^{+}$mesoderm......................................... 106

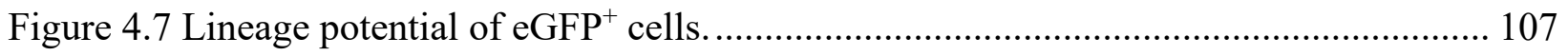




\section{List of Abbreviations}

\begin{tabular}{|c|c|}
\hline ACT A & Activin A \\
\hline ACT Ai & Activin A inhibitor \\
\hline AGM & aorta-gonad mesonephros \\
\hline ALDH & aldehyde dehydrogenase \\
\hline AML & acute myeloid leukemia \\
\hline ATRA & all-trans retinoic acid \\
\hline $\mathrm{BL}-\mathrm{CFC}$ & blast colony-forming cell \\
\hline BMP & bone morphogenic protein \\
\hline CAR & chimeric antigen receptor \\
\hline CFU-S & colony-forming unit, spleen \\
\hline CHIP & clonal hematopoiesis of indeterminant potential \\
\hline DETC & dendritic epidermal $\mathrm{T}$ cell \\
\hline $\mathrm{E}$ & embryonic day \\
\hline EB & embryoid body \\
\hline EHT & endothelial-to-hematopoietic transition \\
\hline EMP & erythro-myeloid progenitor \\
\hline FACS & fluorescence-activated cell sorting \\
\hline FGF & fibroblast growth factor \\
\hline
\end{tabular}




\begin{tabular}{|c|c|}
\hline FGFR & fibroblast growth factor receptor \\
\hline FTOC & fetal thymic organ culture \\
\hline GSI & gamma secretase inhibitor \\
\hline HEC & hemogenic endothelial cell \\
\hline hESC & human embryonic stem cell \\
\hline hPSC & human pluripotent stem cell \\
\hline HPSG & heparan sulphate glycoprotein \\
\hline HSC & hematopoietic stem cell \\
\hline iMEF & irradiated mouse embryonic fibroblast \\
\hline iPSC & induced pluripotent stem cell \\
\hline LMP & lymphoid-primed multipotent progenitor \\
\hline $\mathrm{mESC}$ & mouse embryonic stem cell \\
\hline MI & myocardial infarction \\
\hline MOI & multiplicity of infection \\
\hline MPP & multipotent progenitor \\
\hline NICD & Notch intracellular domain \\
\hline NK & Natural killer \\
\hline NSG & 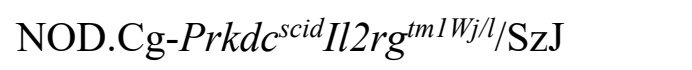 \\
\hline $\mathrm{P}-\mathrm{Sp}$ & para-aortic splanchopleura \\
\hline PCGDTL & primary cutaneous $\gamma \delta \mathrm{T}$ cell lymphoma \\
\hline
\end{tabular}




$\begin{array}{ll}\text { RA } & \text { retinoic acid } \\ \text { RAL } & \text { retinal } \\ \text { ROH } & \text { retinol } \\ \text { s.e.m. } & \text { standard error of the mean } \\ \text { scRNA-seq } & \text { single cell RNA sequencing } \\ \text { SsODN } & \text { single-stranded oligonucleotide } \\ \text { T-reg } & \text { T regulatory } \\ \text { TCR } & \text { T cell receptor } \\ \text { VEGF } & \text { vascular endothelial growth factor } \\ \text { VEGFR } & \text { vascular endothelial growth factor receptor } \\ \text { WNTi } & \text { Wnt inhibitor }\end{array}$




\section{Chapter 1}

\section{Introduction}

\subsection{Hematopoiesis}

The hematopoietic system constitutes a complex network of cells that are disseminated throughout the body to carry out multiple functions, including oxygen transport, elimination of infectious agents and maintenance of vascular integrity, among others. In the adult, the terminally differentiated, functional hematopoietic cells are continually replenished by progenitors, which in turn are generated from hematopoietic stem cells (HSCs) to maintain a constant supply of blood cells throughout life. The regenerative ability of the hematopoietic system was first documented over 70 years ago by studies that demonstrated that the transplantation of spleen or bone marrow cells from donor animals could rescue hematopoietic cell destruction in animals exposed to lethal doses of irradiation (Jacobson et al., 1951; Lorenz et al., 1951). These observations provided the basis for the first bone marrow transplantations in humans (Thomas et al., 1957), which now serves as a curative therapy for numerous hematologic diseases (reviewed in Balassa et al., 2019). Studies aimed at understanding the radiosensitivity of the blood-forming system demonstrated that progenitors contained within the bone marrow give rise to colonies of hematopoietic cells following transplantation into irradiated mice. Through irradiation-induced chromosomal marking, each splenic colony, consisting of cells belonging to the erythroid and myeloid lineages was shown to originate from a single hematopoietic progenitor, known as the colony-forming unit, spleen (CFU-S). The observation that the number of colonies was directly related to the number of cells that were injected established the spleen colony-forming assay as the first method to quantify hematopoietic progenitors (Becker et al., 1963; Siminovitch et al., 1963; Till and McCulloch, 1961). Although these analyses demonstrated that the erythroid and myeloid lineages develop from a common progenitor, their relationship to the lymphoid lineage remained unclear, as lymphocytes were not routinely detected in the splenic colonies. Subsequent studies that followed the fates of transplanted progenitor populations uniquely marked by irradiation-induced DNA damage or retroviral integration sites provided unequivocal evidence that the hematopoietic system develops from multipotent, self-renewing stem cells that display the potential to generate erythroid, myeloid and lymphoid progeny (Dick et al., 1985; Keller et al., 1985; Williams et al., 
1984; Wu et al., 1968). Additional support for the presence of multipotent HSCs has been provided by the use fluorescent-activated cell sorting (FACS) to resolve functionally distinct hematopoietic populations. The identification of several markers has enabled the isolation of single HSCs that provide long-term, multilineage reconstitution following transplantation (Benveniste et al., 2003; Kiel et al., 2005; Osawa et al., 1996). In the mouse, HSCs can be purified using different strategies, including Rhodamine ${ }^{\text {lo }} \mathrm{Ly}^{+} \mathrm{A}^{+} \mathrm{Kit}^{+} \mathrm{B} 220^{-} \mathrm{CD}^{-}$(Benveniste et al., 2003), lineage-negative (B220, CD4, CD8, Gr-1, Mac-1 and Ter119) $\mathrm{Sca}^{+} \mathrm{Kit}^{+} \mathrm{CD} 34^{\mathrm{lo} /-}$ (Osawa et al., 1996) and lineage-negative (B220, CD3, CD5, CD8, Gr-1 and Ter119) $\mathrm{Sca}^{+} \mathrm{Kit}^{+} \mathrm{CD} 150^{+} \mathrm{CD} 48^{-}$(Kiel et al., 2005).

One criticism of these studies is that they all involved transplantation-induced stress to demonstrate the presence of multipotent cells. To demonstrate that multipotent cells are also present and functioning in steady-state hematopoiesis, Sun et al. (2014) developed a doxycycline-inducible hyperactive Sleeping Beauty transposase to track clones by unique DNA transposon integration sites. Pei et al. (2017) developed a complimentary approach that followed the fate of single cells through unique "barcodes" that were generated from a DNA cassette of 10 loxP sites following the activation of Cre recombinase with tamoxifen. The findings from these studies showed that the hematopoietic system is maintained by self-renewing, multipotent cells in vivo.

The approaches pioneered in the mouse were successfully translated to the study of human hematopoiesis. Multilineage engraftment of human hematopoietic cells in irradiated, immunedeficient mice (Kamel-Reid and Dick, 1988) has provided an engraftment assay to trace the potential of different progenitor populations and interrogate the biology of human HSCs. Using FACS-based isolation strategies, Notta et al. (2011) identified a progenitor in human umbilical cord blood defined by $\mathrm{CD} 34^{+} \mathrm{CD} 38^{-} \mathrm{CD} 90^{+} \mathrm{CD} 45 \mathrm{RA}^{-}$Rhodamine ${ }^{\mathrm{lo}} \mathrm{CD} 49 \mathrm{f}^{+}$that could generate long-term multilineage grafts following transplantation. In addition to long-term repopulation assays, several in vitro assays have been also developed to assay hematopoietic progenitor potential, including colony-forming assays and stromal cell-based co-cultures. These different approaches have been used to establish a detailed lineage map of both the mouse and human adult hematopoietic systems with the HSC at the apex of the hierarchy (reviewed in Laurenti and Gottgens, 2018). 


\subsection{The embryonic hematopoietic programs}

Embryonic hematopoiesis consists of distinct programs, including primitive, erythro-myeloid progenitor (EMP), lymphoid-primed multipotent progenitor (LMP) and definitive that differ in their lineage potential and spatiotemporal organization. The first three programs initiate in the yolk sac, whereas definitive hematopoiesis is specified at different sites throughout the developing embryo. In contrast to the adult system, the yolk sac hematopoietic lineages are not derived from an HSC, as they develop prior to its specification. Our understanding of these programs has been informed by studies using model organisms, including the chick (Gallus gallus), quail (Coturnix coturnix), zebrafish (Danio rerio) and mouse (Mus musculus). Unless otherwise indicated, the following text refers to studies of mouse hematopoietic development.

\subsubsection{Primitive hematopoietic program}

The primitive hematopoietic program gives rise to the first hematopoietic cells in embryonic development that appear in the yolk sac of the mouse at embryonic day (E) 7.0 and at day 13 of gestation in the human (Bloom and Bartelmez, 1940; Palis et al., 1999). A hallmark of the primitive hematopoietic program is the emergence of distinct blood islands that consist of primitive erythroblasts surrounded by developing vascular endothelial cells at E7.5 in the mouse and 18.521 days of gestation in the human (Bloom and Bartelmez, 1940; Haar and Ackerman, 1971; Tavian et al., 1999). The primitive hematopoietic program predominantly generates primitive erythrocytes that are distinct from those found at later stages of gestation and in post-natal life. Primitive erythroblasts are large and enucleate only at the final stages of their lifespan, long after their entry into the circulation (Barker, 1968; Kingsley et al., 2004). Additionally, the primitive erythroid lineage displays a unique $\beta$ globin expression pattern characterized by the predominance of the embryonic $\varepsilon \gamma$ and $\beta \mathrm{H} 1$ globins in the mouse and HBE $(\varepsilon)$ in the human (Palis et al., 1999; Peschle et al., 1985; Wong et al., 1986b).

Following their emergence in the yolk sac, primitive erythrocytes appear in the embryo proper, rapidly increase in number between E8.5 and E10.5 and are the dominant contributor to the red blood cell population of the embryo up to E12.5 (Kingsley et al., 2004; McGrath et al., 2003). While the number of primitive erythrocytes remains stable between E12.5 and E16.5, their contribution to the total pool of erythrocytes quickly decreases from $90 \%$ to less than $10 \%$ in that time with a minority persisting until after birth (Kingsley et al., 2004). 
Although mature hematopoietic cells of the primitive hematopoietic program persist until birth, the progenitors of its lineages are only present over a narrow window of development (Palis et al., 1999; Wong et al., 1986a). The transient nature of the primitive erythroid progenitors has been demonstrated through the use of colony-forming progenitor assays that measure their ability to generate small colonies of erythroblasts. These analyses showed that primitive erythroid colonyforming potential was detected in the mouse yolk sac for only 48 hours, between E7.0 and E9.0. The lack of primitive erythroid progenitors in the embryo proper and developing fetal liver, and their presence in the yolk sac of circulation-deficient $N c x 1^{-/-}$mice provided strong evidence that the primitive hematopoietic program is specified in and restricted to the yolk sac (Lux et al., 2008; Palis et al., 1999).

While the primitive hematopoietic program predominantly gives rise to the erythroid lineage, macrophages have also been observed in the yolk sac at early developmental stages (Bloom and Bartelmez, 1940). In line with this, macrophage colony-forming progenitors were detected simultaneously with primitive erythroid colony-forming progenitors in the E7.0 yolk sac (Palis et al., 1999). The appearance of primitive macrophages preceded the detection of monocytes, suggesting that the primitive macrophage lineage does not transition through a monocytic intermediate (Takahashi et al., 1989). This was formally demonstrated through the culture of yolk sacs at different developmental stages with a bone marrow stromal cell line (ST2 cells) supportive of macrophage differentiation. While monocytes were observed in cultures derived from the E10.0 yolk sac, a developmental stage that follows the exhaustion of the primitive hematopoietic program, they were not observed in cultures initiated with the E8.0 yolk sac (Naito et al., 1989). The primitive hematopoietic program also gives rise to mast cells, albeit their progenitors are significantly less numerous than erythroid and macrophage progenitors (Palis et al., 1999). Although the primitive hematopoietic program generates several myeloid lineages, it does not give rise to granulocytes as granulocyte colony-forming progenitors are only observed at E8.5, coincident with the decline of the primitive erythroid lineage (Palis et al., 1999).

In addition to a unique erythroid lineage and restricted myeloid potential, the primitive hematopoietic program also gives rise to megakaryocytes. Clonogenic megakaryocyte progenitors and maturing megakaryocytes are observed in the E7.5 and E8.5 yolk sac, respectively. Consistent with the erythroid and macrophage lineages, the primitive megakaryocytic lineage also differs from its adult counterpart. Primitive megakaryocytes are smaller and their ploidy (DNA content) 
is lower than those of adult megakaryocytes (Xu et al., 2001). In addition, primitive megakaryocytes generate larger platelets than those produced from adult megakaryocytes and do so in a shorter period of time (Tober et al., 2007; Xu et al., 2001).

Primitive hematopoietic progenitors can be isolated from the yolk sac between E7.0 and E8.5 based on the low expression of CD41 and Kit (Ferkowicz et al., 2003; McGrath et al., 2015; Mikkola et al., 2003a). Although no demonstration that single CD4 ${ }^{10} \mathrm{Kit}^{\mathrm{lo}}$ cells give rise to the primitive erythroid, myeloid and megakaryocytic lineages has be made, the detection of progenitors in the E7.5 yolk sac that generate mixed lineage colonies (Tober et al., 2007; Xu et al., 2001 ) indicates that the primitive hematopoietic program is initiated multipotent progenitors.

\subsubsection{Erythro-myeloid progenitor hematopoietic program}

Prior to the loss of primitive erythroid progenitors at E9.0, a second erythroid lineage emerges in the yolk sac at E8.25 that generates erythroblast colonies that express $\beta$ major globin along with low levels of the $\beta$ H1 globin (McGrath et al., 2011; Palis et al., 1999; Wong et al., 1986a). Similar to the primitive erythroid lineage, the progenitors of this erythroid lineage also originate in the yolk sac, as demonstrated through analyses of circulation-deficient $N c x 1^{-/-}$embryos (Lux et al., 2008; McGrath et al., 2015). These progenitors peak in number at E9.5 in the yolk sac, prior to their progressive expansion in the liver and blood at later stages of development (Palis et al., 1999). The daughter erythrocytes are enucleated when they first appear in the blood at E11.5 and increase in frequency and number as the primitive erythroid content in the circulation declines (Kingsley et al., 2004; McGrath et al., 2011). These erythrocytes are significantly smaller than enucleated primitive erythrocytes, but larger than those found in the adult (McGrath et al., 2011).

Consistent with a second wave of hematopoiesis, the number of macrophage progenitors increases in the yolk sac and peaks at E9.5 before appearing in the embryo (Palis et al., 1999). In contrast to primitive macrophages, these macrophage progenitors transition through a monocytic intermediate

(Naito et al., 1989). Mast cell progenitors are also observed in the yolk sac following the attenuation of primitive erythroid progenitors and later appear in the embryo (Palis et al., 1999). While the erythroid, macrophage and mast cell lineages are also generated from the primitive hematopoietic program, granulocyte progenitors are first observed in the yolk sac at E8.5 and peak in number at E9.5 before appearing in the embryo (Palis et al., 1999). These findings, together with a more equitable balance in the number of erythroid and myeloid progenitors (Palis et al., 
1999) provided evidence that this represents a second yolk sac hematopoietic program. This wave has become known as the erythro-myeloid progenitor (EMP) program. EMP hematopoiesis also gives rise to megakaryocytes that generate platelets that are smaller than those derived from the primitive hematopoietic program, but larger than HSC-derived platelets (Tober et al., 2007).

Beyond temporal differences in their emergence, EMPs can be distinguished from primitive hematopoietic progenitors based their expression of several surface markers, including CD41, Kit, CD16/32 (FcyII/III), CD34 and CD45 (Ferkowicz et al., 2003; McGrath et al., 2015; Mikkola et al., 2003a). They also differ from HSCs and lymphoid progenitors, as they do not express Sca1, $\mathrm{CD} 150, \mathrm{Il7r}$ or Flt3. $\mathrm{CD} 41^{+} \mathrm{Kit}^{+} \mathrm{CD} 16 / 32^{+}$EMPs are first observed in the yolk sac at E8.5 and are later found in the circulation and liver at E10.5. Analyses of single $\mathrm{CD} 41^{+} \mathrm{Kit}^{+} \mathrm{CD} 16 / 32^{+}$cells revealed that the EMP program is initiated by multipotent hematopoietic progenitors that generate granulocytic, macrophage, megakaryocytic and erythroid progeny. Despite their multilineage and extensive proliferative potential, EMPs are unable to engraft irradiated adult recipients (McGrath et al., 2015) indicating that they are distinct from HSCs.

Although relatively little is understood about the EMP program in the human, EMP-like erythroid and granulocyte colony-forming progenitors have been observed in the yolk sac at 4 weeks of gestation (Migliaccio et al., 1986). These progenitors appear in the liver by week 5, along with enucleated erythroblasts that express a combination of the embryonic (HBE; $\varepsilon$ ) and fetal (HBG1/2; A $\gamma$ and G $\gamma$ ) $\beta$ globin (Migliaccio et al., 1986; Peschle et al., 1985; Peschle et al., 1984). These findings suggest that an EMP program, analogous to the one present in mouse, may also exist in the human.

\subsubsection{Lymphoid-primed multipotent progenitor hematopoietic program}

The thymic anlage is first colonized by hematopoietic cells at E11.0 (Luis et al., 2016; Moore and Owen, 1967). The scarcity of HSCs $(<3)$ in the embryo prior to E11.0, as determined through limiting dilution repopulation analyses (Gekas et al., 2005; Kumaravelu et al., 2002) suggested that the earliest lymphoid progenitors emerge from the yolk sac independent of HSCs. The detection of $\mathrm{T}$ lymphoid progenitors in the E9.0-E9.5 yolk sac through the use of fetal thymic organ culture (FTOC) or culture with OP9 stromal cells engineered to express the Notch ligand, DL1 provided additional evidence for the presence of an HSC-independent lymphoid program (Huang and Auerbach, 1993; Yoshimoto et al., 2012). Similar to the primitive progenitors and 
EMPs, these T lymphoid progenitors appear to originate in the yolk sac, as they are detectable in this tissue of circulation-deficient $N c x 1^{-/}$embryos. These $\mathrm{T}$ lymphoid progenitors can be distinguished from EMPs by the fact that they express CD144 (vascular endothelial cadherin; VEcad) and lack the expression of CD41 (Yoshimoto et al., 2012).

The first $\mathrm{CD}^{+}$cells are detected in the thymus by E14.0 and their number increases thereafter (Havran and Allison, 1988). The firstborn T lymphocytes show a restricted T cell repertoire (TCR) expressing a single $\mathrm{V}$ gene, $\gamma 3$ and are later found in the skin of adult mice (Havran and Allison, 1988; Kuziel et al., 1987; Stingl et al., 1987). These cells emerge prior to the development of other $\gamma \delta$ and $\alpha \beta$ T lymphocytes (Havran and Allison, 1988) indicating that T lymphoid development is temporally regulated. The early development of the $\mathrm{V} \gamma 3^{+}$population suggested that it may be derived from yolk sac progenitors. Studies by Yoshimoto et al. (2012) confirmed this hypothesis through the demonstration that E9.5 yolk sac progenitors give rise to $\mathrm{V} \gamma 3^{+} \mathrm{T}$ lymphocytes following culture with OP9-DL1 cells. When transplanted into the peritoneal cavity of immunodeficient neonatal mice, the E9.5 yolk sac progenitors generated grafts consisting of $\gamma \delta$ and $\alpha \beta \mathrm{T}$ lymphocytes indicating that this tissue has broad T lymphoid potential.

T lymphoid progenitors and T lymphocytes are present the human thymic rudiment by week 7 of gestation (Farley et al., 2013; Haynes and Heinly, 1995; Haynes et al., 1988a; Haynes et al., 1988b; Park et al., 2020). However, the expression of the V $\gamma 9$ and $V \delta 2$ genes in the liver at week 5 of gestation (McVay and Carding, 1996) highlights that this lineage is present even earlier in development. While $\mathrm{V} \gamma 3^{+} \mathrm{T}$ lymphocytes are the dominate $\mathrm{T}$ lymphocyte population in early mouse development (Havran and Allison, 1988), $\mathrm{V} \gamma 9^{+} \mathrm{V} \delta 2^{+} \mathrm{T}$ lymphocytes are the most abundant $\gamma \delta \mathrm{T}$ lymphocyte population in the liver and peripheral blood of the human fetus (Dimova et al., 2015; McVay and Carding, 1996). This population is detected prior to the appearance of $\alpha \beta \mathrm{T}$ lymphocytes in the thymus (Haynes et al., 1988b) recapitulating the temporal allocation of $\mathrm{T}$ lymphoid fates observed in the mouse.

In addition to $\mathrm{T}$ lymphoid potential, progenitors in the E9.0 yolk sac can give rise to $\mathrm{B}$ cells following culture with OP9 stromal cells suggesting an HSC-independent source of this lineage, as well. Like the yolk sac T lymphoid progenitors, B cell potential is contained within the CD144 ${ }^{+} \mathrm{CD}^{-} 1^{-}$population (Yoshimoto et al., 2011). These progenitors are the presumptive source of B cells that are found in the liver at E11.0 (de Andres et al., 2002; Montecino-Rodriguez et al., 
2006). Transplantation of E9.0 yolk sac cells into the peritoneal cavity of immunodeficient neonatal mice gave rise to grafts of B1 B cells in the peritoneal cavity and spleen, whereas those from the E15.0 fetal liver and HSCs generated both B1 and B2 B cells. (Hadland et al., 2017; Yoshimoto et al., 2011). These different B cell subsets are functionally distinct. B1 cells secrete IgM antibodies independent of $\mathrm{T}$ lymphocyte stimulation, reside in the pleural and peritoneal cavities, marginal zones of the spleen and lymph nodes, and express CD5 and CD11b (reviewed in Baumgarth, 2017). B1 cells constitute a minor fraction of the B cell pool and are outnumbered by $\mathrm{B} 2$ cells. These B2 cells do not express IgM and generate antigen-specific antibodies following stimulation by $\mathrm{T}$ lymphocytes (reviewed in Vale et al., 2015). In the human, $\mathrm{CD} 20^{+} \mathrm{CD} 27^{+} \mathrm{CD} 43^{+} \mathrm{CD} 70^{-}$cells isolated from the peripheral blood are thought to represent $\mathrm{B} 1$ cells, as they spontaneously secrete IgM (Griffin et al., 2011b). However, differences in sample preparation, FACS gating strategies and interpretation of molecular analyses have led to questions about whether this population truly contains B1 cells (Covens et al., 2013a; Covens et al., 2013b; Descatoire et al., 2011; Griffin et al., 2011a; Li et al., 2013; Perez-Andres et al., 2011).

Natural killer (NK) cell progenitors, detected by their ability to generate NK cells following culture with OP9 stromal cells are present in the yolk sac as early as E8.5 (Dege et al., 2020). Unlike the $\mathrm{B}$ and $\mathrm{T}$ lymphoid progenitors that were restricted to the $\mathrm{CD} 41^{-}$population (Yoshimoto et al., 2012), NK cell potential is contained within the $\mathrm{CD} 41^{+} \mathrm{Kit}^{+} \mathrm{CD} 16 / 32^{+}$EMP population. NK cells later appear in the liver at E13.5 and increase in number thereafter. The fetal NK cells are more granular than those found in the adult and express higher levels of CD107a following stimulation. As CD107 is associated with degranulation, these observations suggest that fetal NK cells are more cytotoxic than adult NK cells (Dege et al., 2020).

NK cells are detected in human livers at 6 weeks of gestation (Phillips et al., 1992). However, in contrast to those in the mouse, human fetal NK cells appear to be less cytotoxic than those found in the newborn and adult (Ivarsson et al., 2013; Phillips et al., 1992).

The spatial and temporal overlap between the emergence of EMPs and B, T and NK lymphoid progenitors raises the possibility that these lineages are generated from a common multipotent hematopoietic progenitor. The identification of $\mathrm{CD} 5^{+} \mathrm{Kit}^{+} \mathrm{Il} 7 \mathrm{ra}^{+} \mathrm{Flt} 3^{+}$progenitors in the E11.5 liver that can generate the granulocytic, macrophage, B and T lymphoid lineages supports this

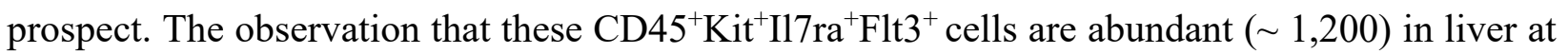


E11.5 (Boiers et al., 2013), a stage where exceptionally few HSCs $(\sim 5)$ are present in the whole embryo (Gekas et al., 2005; Kumaravelu et al., 2002) suggests that these progenitors are derived from progenitors specified in the yolk sac. Although the $\mathrm{CD} 45^{+} \mathrm{Kit}^{+} \mathrm{Il} 7 \mathrm{ra}^{+} \mathrm{Flt} 3^{+}$progenitors do not efficiently give rise to the erythroid or megakaryocytic lineages, erythroid and megakaryocytic progenitors are present in the $\mathrm{CD} 45^{+} \mathrm{Kit}^{+} \mathrm{Il} 7 \mathrm{ra}^{-}$population found in the liver at this time (Boiers et al., 2013). One interpretation of these findings is that the $\mathrm{CD}^{+} 5^{+} \mathrm{Kit}^{+} \mathrm{Il} 7 \mathrm{ra}^{+} \mathrm{Flt} 3^{+}$and $\mathrm{CD} 5^{+} \mathrm{Kit}^{+} \mathrm{Il} 7 \mathrm{ra}^{-}$populations are derived from multipotent progenitors present at earlier stages of development. The identification of the $\mathrm{CD} 45^{+} \mathrm{Kit}^{+} \mathrm{IL} \mathrm{ra}^{+} \mathrm{Flt}{ }^{+}$progenitors with lymphoid and myeloid, but not erythroid or megakaryocytic potential has provided the basis the concept of a third yolk sac hematopoietic program, which has become known as the lymphoid-primed multipotent progenitor (LMP) program.

\subsubsection{Definitive hematopoietic program}

The definitive hematopoietic program generates the HSCs that go on to support multilineage hematopoiesis throughout life. The early observations that the yolk sac is the first site of hematopoietic development led to the assumption that HSCs are also specified in this extraembryonic site. However, this view was challenged by studies of Dieterlen-Lievre (1975) who tracked the hematopoietic contribution of the yolk sac and embryo proper over time in chimeric embryos generated by grafting developing quail embryos onto chick yolk sacs prior to vascularization. Soon after grafting, chick erythroid cells predominated and were detected in the yolk sac, as well as the spleen of the embryo. With time, the contribution of the yolk sac (chick) declined and that of the embryo (quail) increased. Notably, the majority of the lymphoid cells in the developing thymus were of quail origin. The findings from this landmark study provided strong evidence for an intra-embryonic source of definitive hematopoiesis. Following these observations, numerous studies have investigated the site of HSC development and have demonstrated that HSCs are specified at multiple vascular sites within the embryo, including the head, placenta, umbilical arteries and the region that includes the aorta-gonad mesonephros (AGM) (Gekas et al., 2005; Li et al., 2012; Muller et al., 1994; Ottersbach and Dzierzak, 2005). The AGM is by far the best characterized site and within this region of the embryo, detailed analyses have shown that HSCs emerge from the ventral wall of the dorsal aorta (Bertrand et al., 2010a; Boisset et al., 2010; Kissa and Herbomel, 2010). Limiting dilution repopulation analyses have shown that a small number of HSCs ( $<3$ per embryo) are detected as early as E10.5. Their number dramatically increases to 
more than 250 over the next 48 hours. By E12.0 HSCs are present in the circulation and developing liver (Gekas et al., 2005; Kumaravelu et al., 2002). Although newly formed HSCs share the expression of CD41, CD45 and Kit with EMPs and LMPs, they differ in that they also express Ly6a (Sca1) (de Bruijn et al., 2002; Ferkowicz et al., 2003; Sanchez et al., 1996). While transplantation studies that used adult recipient mice only detected HSCs in the embryo proper (Cumano et al., 2001; Medvinsky and Dzierzak, 1996; Müller et al., 1994), it remained a possibility that the yolk sac also contains a less mature version of these cells that are unable to function in the adult environment. This question was addressed by the studies of Yoder and colleagues (1997; 1997a; 1997b), who transplanted cells from the E9.0 yolk sac or embryo proper into the livers of neonatal mice, reasoning that this site would provide a more "fetal" environment to support the development of any HSCs. The strategy worked, as both the yolk sac and embryo proper provided long-term multilineage engraftment in the recipients that grew from the transplanted neonates. When transplanted into secondary adult recipients, marrow cells from the primary engrafted mice provided long-term, multilineage reconstitution (Yoder and Hiatt, 1997; Yoder et al., 1997a) indicating that the yolk sac and embryo proper-derived HSCs had matured to the stage at which they could function in an adult environment. The findings from these studies provided the basis for the notion of a pre-HSC, a progenitor that requires maturation to be able to engraft an adult animal. This concept has been studied by several investigators who have developed OP9 stromal cell or AGM-derived endothelial cell-based co-culture systems to promote the HSC maturation. With these strategies, it has been possible to demonstrate that the cells in the E8.5-9.5 para-aortic splanchnopleura (P-Sp), the precursor of the AGM, which are unable to directly engraft adult animals, acquire this potential following 6 to 7 days of culture with the appropriate cellular support (Hadland et al., 2015; Matsuoka et al., 2001; Rybtsov et al., 2014). These platforms allowed for the identification of the following stages of HSC maturation, known as pro-HSCs, type I and type II pre-HSCs. These stages are distinguished by the expression of CD41, CD43 and CD45. proHSCs: $\mathrm{CD} 144^{+} \mathrm{CD} 41^{+} \mathrm{CD} 43^{-\mathrm{CD}} 45^{-}$, type I pre-HSCs: CD $144^{+} \mathrm{CD} 41^{+} \mathrm{CD} 43^{+} \mathrm{CD} 45^{-}$and type II preHSCs: $\mathrm{CD} 144^{+} \mathrm{CD} 41^{+} \mathrm{CD} 43^{+} \mathrm{CD} 45^{+}$. While allowing for the identification of these developmental stages, culture with OP9 stromal cells does not fully recapitulate the neonatal environment, as this format is unable to support the development of HSCs capable of adult repopulation from the E9.5 yolk sac (Rybtsov et al., 2014). 
Limiting dilution repopulation analyses of human embryo tissues suggest that the AGM contains a single HSC between days 32 and 41 of gestation (Ivanovs et al., 2011). As in the mouse, the placenta also contains HSCs at early stages of gestation (Robin et al., 2009). Using in vitro colonyforming analyses to approximate HSC potential, the first hematopoietic progenitors detected in the human AGM were found to express CD144, CD34, KIT and CD90, but not CD38 and CD45RA (Ivanovs et al., 2014). If the in vitro colony-forming progenitor assay is indicative of HSCs, these observations would suggest that the surface marker profile of the first-born HSCs is similar to those found in fetal liver and cord blood.

\subsubsection{Contribution to the adult hematopoietic system}

Although HSCs sustain multilineage hematopoiesis throughout life, the other embryonic programs do contribute to the post-natal hematopoietic system. The contribution of the primitive and EMP programs to populations of tissue-resident macrophages, specialized immune cells that regulate tissue homeostasis (reviewed in Varol et al., 2015) was first recognized following the demonstration that microglia, the tissue-resident macrophage of the brain, appear this tissue rudiment as early as E8.5 (Alliot et al., 1999). The origin of the microglia was further characterized by lineage tracing studies that showed that the majority these cells are generated from progenitors that expressed Runxl in the yolk sac between E7.0 and E8.0 (Ginhoux et al., 2010). The contribution of the yolk sac hematopoietic programs to microglia, together with the observation that macrophages are broadly disseminated across multiple tissues in the E10.5 embryo (Schulz et al., 2012) prompted further exploration to determine whether other tissue-resident macrophages develop from early embryonic progenitors. Lineage tracing of Csflr-expressing cells, which labels hematopoietic progenitors and myeloid cells, formally demonstrated that tissue-resident macrophages in the skin, liver, spleen, pancreas, lung and kidney are specified from progenitors present in the yolk sac at E8.5 (Gomez Perdiguero et al., 2015; Schulz et al., 2012). In contrast, lineage tracing of Flt3-expressing cells, which represent the progeny of HSCs (Boyer et al., 2011), labelled few tissue-resident macrophages in these organs (Gomez Perdiguero et al., 2015). The HSC-independent origin of tissue-resident macrophages was supported by studies that showed that HSC transplantation in adult mice reconstituted the HSC, myeloid progenitor and monocyte populations, but not tissue-resident macrophages, which remained of recipient origin in several organs (Gomez Perdiguero et al., 2015). This work was complemented by parabiosis experiments that demonstrated that tissue-resident macrophages are minimally replaced by non-host cells, even 
when a common circulation is shared by two animals (Epelman et al., 2014; Hashimoto et al., 2013). Interestingly, the extent of non-host contribution to the tissue-resident macrophage populations varied between organs (Bain et al., 2014; Gomez Perdiguero et al., 2015; Hashimoto et al., 2013) suggesting that the contribution of yolk sac-derived progenitors to tissue-resident macrophage populations in the adult is organ-dependent.

In addition to tissue-resident macrophages, evidence exists that subpopulations of adult mast cells are also derived from yolk sac progenitors. Lineage tracing of Cdh5 (CD144; Ve-cadherin)expressing cells at E7.5, which labels primitive and EMP-derived progenitors, but not the HSC lineage, revealed that a large population of skin mast cells in the newborn are derived from early embryonic progenitors. While the proportion of E7.5 Cdh5-traced mast cells in the skin decreased with age, it increased in the peritoneum increased over time (Gentek et al., 2018a). Lineage tracing of Runx 1-expressing cells has also uncovered the contribution of E8.5 progenitors to mast cells in connective tissues (tongue, pleural and peritoneal cavities) of the E18.5 mouse (Li et al., 2018).

Although the majority of studies have focused on the analysis of subsets of myeloid cells that are generated from the primitive and EMP programs, evidence exists that the LMP program also gives rise to lymphocytes that function in the adult. This concept was supported by the findings of Yoshimoto et al. (2012) that showed that T lymphoid progenitors generated from cells in the E9.5 yolk sac provide long-term thymic and peripheral $\mathrm{T}$ lymphocyte engraftment in immunodeficient recipient mice. Analyses of the T lymphoid populations in these engrafted mice showed that these yolk sac progenitors give rise to $\mathrm{FoxP}^{+} \mathrm{T}$ regulatory (T-reg) cells in the spleen. Taken together with the observation that the fetus contains large numbers of T-reg cells (Michaelsson et al., 2006), these findings suggest that the yolk sac may be a potent source of T-reg cells. The transplantation of yolk sac-derived T lymphoid progenitors to immunodeficient mice also provided robust $\gamma \delta \mathrm{T}$ lymphocyte reconstitution in the liver, unlike those transplanted with P-Sp-derived T lymphoid progenitors (Yoshimoto et al., 2012). The rescue of a specialized population of $\mathrm{V} \gamma 3^{+} \mathrm{T}$ lymphocytes (dendritic epidermal T cells; DETCs) in the skin of athymic nude mice following the transplantation of E14.0 thymocytes (Havran and Allison, 1990) raised the possibility that DETCs are also generated by the LMP program. This concept was supported by the demonstration that the E9.5 yolk sac generates $\mathrm{V} \gamma 3^{+} \mathrm{T}$ lymphocytes following culture with OP9-DL1 cells and lineage tracing studies that showed that progenitors expressing Cdh5 and Runxl at E7.5 contribute a subpopulation of DETCs in the adult mouse (Gentek et al., 2018b; Yoshimoto et al., 2012). 
An embryonic origin for the B cell lineage was supported by the demonstration that adult HSCs do not efficiency give rise to B1 cells (Barber et al., 2011). E9.5 yolk sac progenitors, on the other hand, were able to efficiently reconstitute the peritoneal cavity B1 cell population in recipient animals (Yoshimoto et al., 2011). The origin of the B1 cell lineage has been further investigated by Hadland et al. (2017) who showed that single E9.5 P-Sp progenitors could generate both B1 cells and HSCs following 7 days of culture with AGM-derived endothelial cells. While these findings seem to suggest two origins of B1 cells, it is possible that the B1 lineage and definitive hematopoiesis segregate early from a common pro-HSC. Beyond this point, B1 cells would persist and function independently of definitive hematopoiesis. Collectively, these findings provide strong evidence that several immune cell populations found in adult tissues develop from the progenitors of the primitive, EMP and LMP programs. 


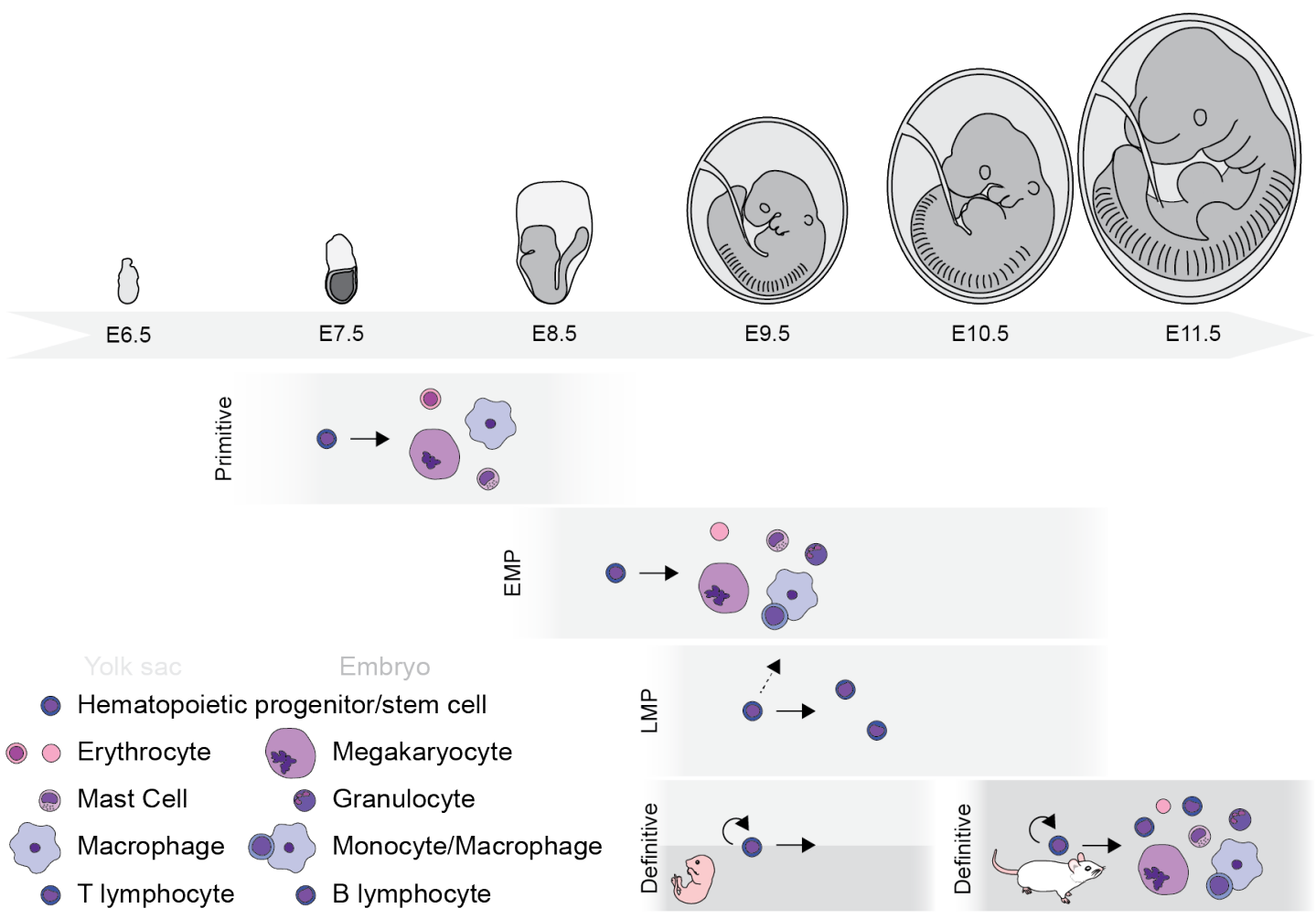

Figure 1.1 Mouse embryonic hematopoietic development.

Mouse embryonic hematopoietic development consists of distinct programs that differ in their spatiotemporal organization and lineage potential. The primitive hematopoietic program is the first to emerge at E7.0 and originates in the yolk sac (light grey shading). The primitive hematopoietic program is transient in nature and gives rise to the primitive erythroid, macrophage, megakaryocytic and mast cell lineages. Prior to the exhaustion of the primitive hematopoietic program, the erythro-myeloid progenitor (EMP) program emerges in the yolk sac at E8.25. It generates erythroid, monocyte/macrophage, megakaryocytic, mast cell and granulocytic progeny. The macrophages generated from the primitive and EMP programs give rise to tissue-resident macrophages. Progenitors of the lymphoid-primed multipotent progenitor (LMP) program are also detected in the yolk sac at E9.0 and generate B and T lymphocytes. Clonal analyses that showed that single cells generate lymphocytes and myeloid cells indicates that the EMP and LMP programs may be linked (dashed arrow). The first HSCs are observed at E9.0 in the yolk sac and embryo (dark grey shading). While the HSCs at E9.0 only reconstitute the hematopoietic system of neonatal recipients following transplantation, HSCs at E10.5 are also able to reconstitute adult recipients, similar to HSCs isolated from fetal liver, cord blood and bone marrow. 


\subsection{Progenitors of embryonic hematopoietic programs}

\subsubsection{Hemangioblast}

Early analyses of blood island formation in the chick and mouse described the close association between the hematopoietic and endothelial fates raising the possibility that these lineages develop from a common precursor, known as the hemangioblast (Haar and Ackerman, 1971; Murray, 1932; Sabin, 1920). Formal demonstration of the existence of the hemangioblast came through the analyses of populations differentiated from mouse embryonic stem cells (mESCs). These studies identified a mESC-derived progenitor that generated colonies of immature blast cells, when cultured in methylcellulose supplemented with appropriate hematopoietic cytokines. When expanded in liquid culture, single blast colonies that were shown to represent clones, gave rise to both hematopoietic and endothelial progeny (Choi et al., 1998). Further characterization of their hematopoietic potential revealed that individual blast colonies generated both primitive erythroid cells characterized by the predominant expression of the $\beta \mathrm{H} 1$ globin and a second erythroid lineage that expressed the $\beta$ major globin and low levels of the $\beta \mathrm{H} 1$ globin. This second erythroid lineage was considered to represent definitive erythroid cells at the time (Kennedy et al., 1997). However, with the subsequent identification and characterization of the EMP program, it is likely that these "definitive" erythroid cells represent the EMP erythroid lineage, given the persistent expression of the $\beta \mathrm{H} 1$ globin. These findings provided evidence that the yolk sac hematopoietic programs develop from a common progenitor, known as the blast colony-forming cell (BL-CFC), the in vitro equivalent of the hemangioblast.

BL-CFCs represent a transient progenitor that emerges prior to the onset of hematopoiesis and persists over a narrow window (days 3.0 to 4.25) in mESC-derived cultures (Kennedy et al., 1997). Early in development, Flk1 (Kdr) is expressed on lateral plate mesoderm, as it egresses from the primitive streak (Yamaguchi et al., 1993). Analyses of Flk1 expression at early stages of mESC differentiation showed that Flk1 is present on a small fraction of cells, including BL-CFCs (Faloon et al., 2000; Fehling et al., 2003). To further track the developmental origin of the BL-CFC, Fehling et al. (2003) engineered a mESC to express GFP from the Brachyury (T) locus, a gene expressed in the primitive streak and early mesoderm of the mouse embryo (Wilkinson et al., 1990). These studies showed that BL-CFCs co-express Flk1 and Brachyury, indicating that these progenitors represent a developmental stage at the transition from the primitive streak to mesoderm. Consistent 
with this interpretation, BL-CFCs were found to lack the expression of hematopoietic and endothelial lineage markers, including CD34, CD31 and Kit (Fehling et al., 2003; Kabrun et al., 1997). Analyses of E7.5 Brachyury-GFP mouse embryos identified the presence of $\mathrm{Flk1}^{+}$Brachyury-GFP ${ }^{+} \mathrm{BL}-\mathrm{CFCs}$ with hematopoietic and endothelial potential, similar to those generated from mESCs (Huber et al., 2004). These embryonic BL-CFCs were largely restricted to the posterior primitive streak region of the embryo (Huber et al., 2004), an observation consistent with the interpretation that they represent a progenitor in transition from the primitive streak to mesoderm stage of development. These findings also provided evidence that yolk sac hematovascular commitment occurs at an early stage of development. Although BL-CFCs display both primitive and EMP potential, lineage fates associated with the LMP and definitive hematopoietic programs have not been associated with this progenitor.

\subsubsection{Hemogenic endothelial cells}

In addition to the close association of the hematopoietic and endothelial lineages in the blood islands of the yolk sac, analyses of the AGM revealed the presence of clusters of hematopoietic cells positioned next to the endothelial cell lining of the developing aorta. These observations led to speculation that these hematopoietic cells were generated from a subpopulation of endothelial cells known as hemogenic endothelial cells (HECs) through a process known as endothelial-tohematopoietic transition (EHT) (Jordan, 1917). Support for this concept was provided by studies that showed that populations isolated from the E8.5 to E10.5 yolk sac and embryo proper based on the expression of CD144 (CD144 ${ }^{+} \mathrm{CD} 45^{-}$Ter119- cells) generated erythroid, myeloid and lymphoid progeny following 7 days of culture on type IV collagen or with OP9 stromal cells (Nishikawa et al., 1998a; Nishikawa et al., 1998b). Jaffredo et al. (1998) provided evidence that EHT occurs in situ by injecting acetylated low-density lipoprotein into the chick heart and showing that the labeling of aortic endothelial cells precedes its detection in the hematopoietic clusters. Live imaging of the aorta of the developing zebrafish afforded better resolution of the mechanism of EHT and showed that it is a dynamic process whereby a subset of $k d r l$ (the zebrafish orthologue of Flk1)-expressing cells integrated in the aorta wall progressively egress into the aortic lumen to form hematopoietic clusters, in the absence of cell division. This transition was associated with the upregulation of the hematopoietic markers $c d 41, c d 45$ and $c m y b$, and the downregulation of endothelial markers $k d r l$ and $c d h 5$ (Bertrand et al., 2010a; Kissa and Herbomel, 2010). Imagingbased analyses of the mESC-derived populations and the E10.0 mouse AGM showed similar 
dynamic transitions from HECs to hematopoietic cells indicating that EHT is conserved across species (Boisset et al., 2010; Eilken et al., 2009).

Although the focus of the majority of the HEC and EHT studies are on the AGM and the definitive hematopoietic program, HECs that give rise to erythroid, myeloid and lymphoid progeny have also been identified in the E9.5 yolk sac (Nishikawa et al., 1998a; Nishikawa et al., 1998b; Yoshimoto et al., 2011; Yoshimoto et al., 2012) indicating that the EMP and LMP programs also undergo EHT. To determine if these HEC populations are different, Chen et al. (2011) used Cbf $\beta$-deficient embryos, which lack EMP and definitive hematopoiesis (Wang et al., 1996b). Their study provided evidence that EMP and definitive HECs are distinct based on the findings that the EMP, but not definitive hematopoietic program could be restored in $\mathrm{Cbf}^{-/-}$embryos through its expression in cells expressing Tek (Tie2), which is a receptor tyrosine kinase found in HECs and endothelial cells (Dumont et al., 1992; Tang et al., 2010). In contrast, only the definitive hematopoietic program was rescued through $\mathrm{Cbf} \beta$ expression in Ly6a (Sca1)-expressing cells (Chen et al., 2011). Additional differences were identified by molecular analyses, which showed that $L y 6 a, T c f 7, M s x 2$ and Tfap $2 a$ were expressed at higher levels in the E10.5 embryonic CD $31^{+}$Runxl-GFP ${ }^{+}$population than that in yolk sac, which contain definitive and EMP HECs, respectively (Gao et al., 2018). One concern with this approach is that embryonic population contained 6-fold more HECs than the yolk sac allowing for the possibility that the differences in gene expression are solely due to differences in the purity of the populations between the two samples. The identification of molecular differences between these HEC populations will depend on the identification of markers that enable the isolation and purification of them from related endothelial cells.

While it is clear that HECs are progenitors of the EMP, LMP and definitive hematopoietic programs, there is emerging evidence that suggests the primitive hematopoietic program also transitions through a HEC intermediate. This was provided by Stefanska et al. (2017), who studied the hematopoietic differentiation of mESCs engineered to express eGFP under the control of the $\beta H 1$ promoter and found that cells expressing Kit and Tie2 give rise to $\beta H 1-\mathrm{eGFP}^{+}$cells and primitive erythroid colony-forming progenitors. However, these cells also expressed the hematopoietic marker CD41, still allowing for possibility that the primitive hematopoietic program uniquely generates hematopoietic cells in the absence of a HEC intermediate. 


\subsubsection{Relationship between the embryonic hematopoietic progenitors}

Given the demonstration that the hemangioblast and HECs are both progenitors of the embryonic hematopoietic programs, their relationship came into question. The hemangioblast and HECs were linked by the studies of Lancrin et al. (2009) and Eilken et al. (2009) that followed the development of Flk $1^{+}$populations containing BL-CFCs (Faloon et al., 2000; Fehling et al., 2003). Lancrin et al. (2009) showed that mESC-derived Flk1 ${ }^{+}$mesoderm gives rise to $\mathrm{Kit}^{+} \mathrm{Tie}^{+} \mathrm{CD}^{-} 1^{-}$cells that in turn generate $\mathrm{CD}_{4} 1^{+}$hematopoietic cells. This lineage relationship was also shown to be intact with embryonic cells. Through imaging-based analyses Eilken et al. (2009) demonstrated that single Flk1 ${ }^{+} \mathrm{CD} 144^{-} \mathrm{CD} 41^{-}$cells isolated from the E7.5 mouse give rise to HECs prior to hematopoietic cells. 


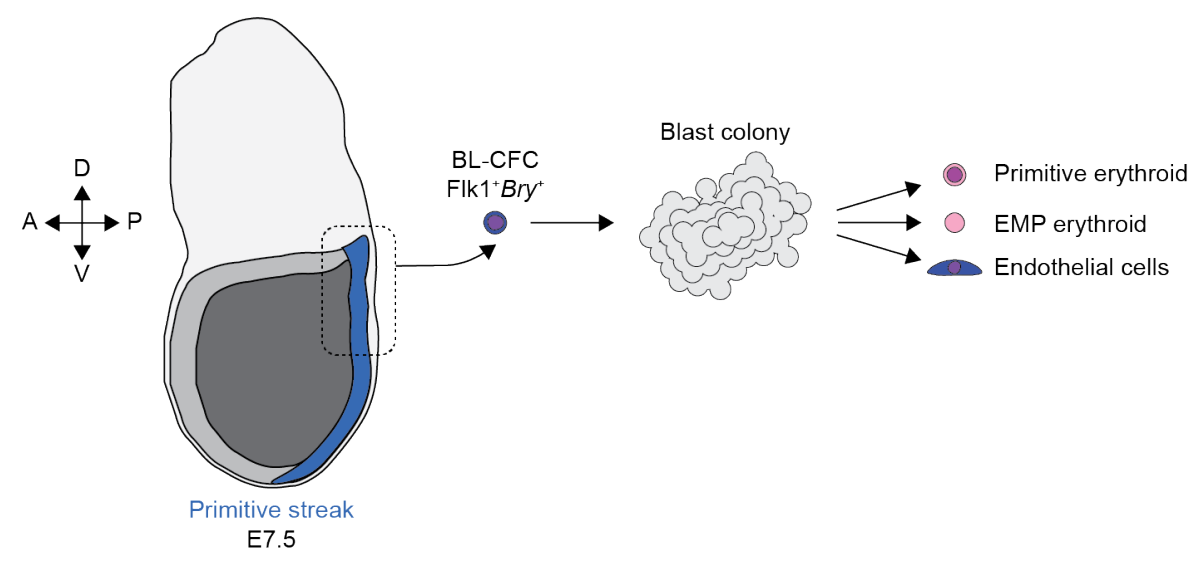

Figure 1.2 The hemangioblast.

A subset of mesoderm in the posterior (P) primitive streak of the E7.5 mouse that expresses Flk1 and Brachyury gives rise to colonies of immature cells (blasts) when cultured in semi-solid methylcellulose. The hemangioblast (blast colony-forming cell, BL-CFC) represents a common ancestor for the hematopoietic and endothelial lineages, as blast colonies derived from single cells generate the primitive erythroid, EMP erythroid and endothelial cells. P: posterior; A: anterior; D: dorsal; V: ventral. 


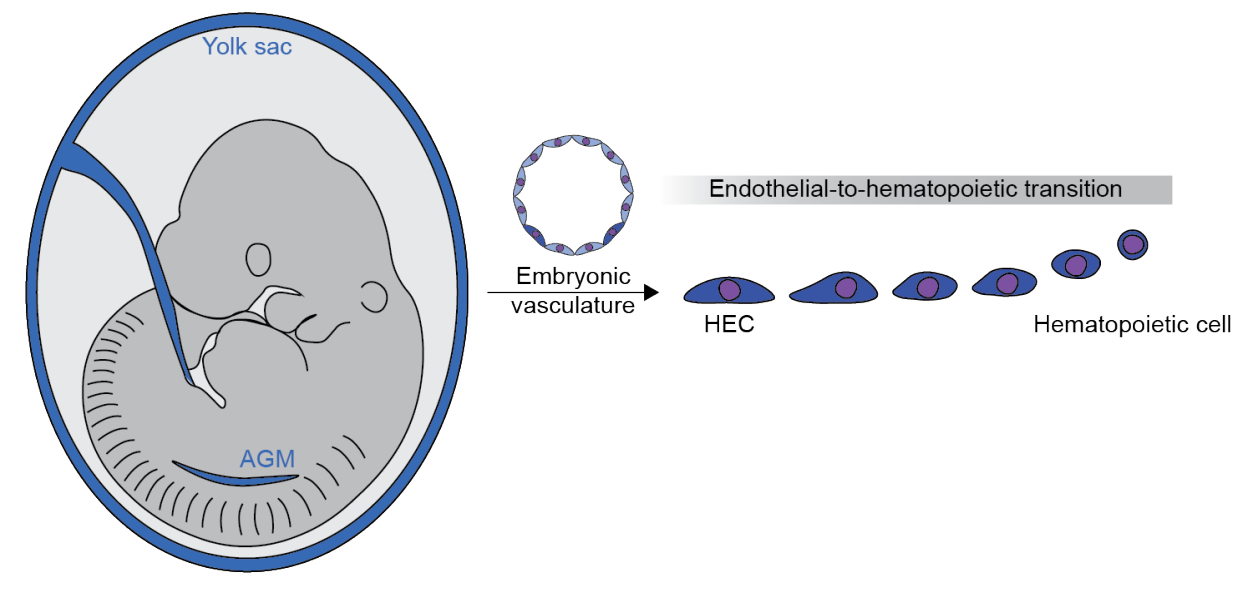

Figure 1.3 Hemogenic endothelial cells.

HECs are observed at several sites in the embryonic vasculature, including in the yolk sac and AGM. These cells undergo a stereotyped endothelial-to-hematopoietic transition, whereby HECs differentiate to become hematopoietic cells as they egress into the lumen of the vessel. 


\subsection{Regulation of embryonic hematopoietic development}

In order to generate hematopoietic cells from hPSCs, it is essential to understand the regulatory mechanisms that guide hematopoietic commitment at different developmental stages. Furthermore, the identification of cell signaling pathways and transcription factors that are specific to the different embryonic hematopoietic programs provides one approach to distinguish them in vitro.

\subsubsection{Signaling pathways}

\subsubsection{Nodal and Activin A signaling}

Nodal and Activin A are part of the TGF $\beta$ superfamily of morphogens and are essential regulators of early developmental processes. Nodal undergoes proteolytic cleavage to generate a functional ligand, while Activin A is formed by Inhibin $\beta$ a dimers. These ligands signal through type II and type I serine-threonine kinase receptors. For type I receptor specificity, Nodal also requires the coreceptor, $\mathrm{Cfc} 1$. The ligand-receptor interaction leads to the phosphorylation of downstream target Smad proteins $(\operatorname{Smad} 2, \operatorname{Smad} 3)$, which in turn interact with other transcription factors in the nucleus to regulate gene expression (reviewed in Pauklin and Vallier, 2015; Shen, 2007).

The indication that this pathway may play a role in early embryonic development was provided by expression studies that showed that Nodal is initially expressed in the node of the anterior primitive streak of embryos (Zhou et al., 1993). The demonstration that Nodal-deficient embryos fail to produce a stereotypic primitive streak and do not establish proper proximal-distal and anteriorposterior axes in the embryo formalized its importance in early fate specification (Conlon et al., 1994). More detailed studies in the amphibian and zebrafish provided evidence that different levels of Nodal and Activin signaling promoted the different cell fates (Gurdon et al., 1994; Thisse et al., 2000).

Studies in both mouse and human PSCs have shown that signaling through this pathway also plays a pivotal role in the early stages of lineage specification in vitro. In the majority PSC studies, Activin A is used in place of Nodal, as it binds the same receptors, is far less costly and shows better activity in vitro. A requirement for Nodal/Activin A signaling in PSC differentiation was first documented using Brachyury-GFP mESCs. The findings from these studies showed that Activin A was essential for the formation of a Brachyury ${ }^{+}$primitive streak and that different levels 
of signaling induce different cell fates, where high and low levels of Activin A induce endoderm and mesoderm, respectively (Gadue et al., 2006; Kubo et al., 2004). Studies with hPSCs have shown that the function of this pathway is conserved across species. As was the case in the mouse, the lineage outcome in hPSC differentiation was dependent on the concentration of Activin A that was used (D'Amour et al., 2005; Kattman et al., 2011; Kennedy et al., 2012). While it is well established that low concentrations of Activin A induce mesoderm, more recent studies have shown that small differences in the concentration of Activin A have large consequences on fate specification. Lee et al. (2017) showed that nanogram per millilitre differences in Activin A promote different cardiomyocyte fates, where $6 \mathrm{ng} / \mathrm{mL}$ and $2 \mathrm{ng} / \mathrm{mL}$ of Activin A induced mesoderm fated to the ventricular and atrial cardiomyocyte fates, respectively.

\subsubsection{Bone morphogenic protein signaling}

Bone morphogenic proteins (Bmps) are part of the TGF $\beta$ superfamily and are critical regulators of embryonic development and adult homeostasis. Bmps are further classified into the following groups based on structural similarity: 1) Bmp2 and Bmp4, 2) Bmp5, Bmp7, Bmp6, Bmp8a and Bmp8b, 3) Bmp9 and Bmp10; Bmp12, Bmp13 and Bmp14, 4) Bmp15 and Gdf9, 5) Bmp3 and Gdf10. Following their translation, Bmps undergo successive rounds of peptide processing and then dimerize to become active. Bmps signal through type II and type I serine-threonine kinase receptors leading to the phosphorylation of downstream target Smad proteins (Smad1, Smad5 and Smad8). These phosphorylated Smads then coordinate the transcription of target genes in conjunction with other transcription factors (reviewed in Katagiri and Watabe, 2016; Wang et al., 2014).

A developmental role for Bmps was suggested by early studies that showed that Bmp2 and Bmp4 are expressed in the early embryo from E7.5 and E6.5, respectively (Johansson and Wiles, 1995; Winnier et al., 1995; Zhang and Bradley, 1996). Bmp2 is first expressed in the anterior neural folds and pre-cardiac mesoderm, while Bmp4 is expressed in the posterior primitive streak (Winnier et al., 1995; Zhang and Bradley, 1996). Genetic analyses provided proof that these Bmps are required for embryonic development. Bmp2-null mice show severe cardiovascular defects and amnion/chorion malformation (Zhang and Bradley, 1996), whereas Bmp4-deficient mice fail to undergo gastrulation and do not form Brachyury ${ }^{+}$cells or mesoderm derivatives (Winnier et al., 1995). Studies using the mESC differentiation model provided an opportunity to investigate the 
stage-specific role of Bmp signaling. The findings from these studies showed that Bmp4 is required for the generation of $\mathrm{Flk1}^{+}$mesoderm, but for not the primitive streak (Johansson and Wiles, 1995; Nostro et al., 2008). Given its role in mesoderm induction, Bmp signaling is essential for the specification of the hematopoietic fates from mESCs (Lengerke et al., 2008; Nostro et al., 2008; Pearson et al., 2008). As is the case with the differentiation of mESCs, BMP signaling is required for mesoderm induction and hematopoietic development from hPSCs (Kennedy et al., 2007; Pick et al., 2007; Zhang et al., 2008).

\subsubsection{Fibroblast growth factor signaling}

Fibroblast growth factors (Fgfs) represent a large class of morphogens that regulate embryogenesis. Like Bmps, Fgfs are also classified by sequence homology. The majority of Fgfs are secreted and signal through heparan sulphate glycoprotein (HPSG) and Fgf receptor (Fgfr; Fgfr1, Fgfr2, Fgfr3, Fgfr4) complexes. Despite the limited number of receptors that bind Fgfs, ligand specificity is conferred through differential expression between cell types and alternative splicing of the receptors. The ligand-receptor interaction leads to the phosphorylation of the intracellular domains of the Fgfrs, which phosphorylate target proteins, such as Plc $\gamma$, Pi3k/Akt and Mek/Erk to initiate downstream signaling (reviewed in Brewer et al., 2016; Dorey and Amaya, 2010; Turner and Grose, 2010).

Genetic analyses revealed a requirement of Fgfr1 for primitive streak formation and mesoderm induction (Deng et al., 1994; Yamaguchi et al., 1994). Although embryonic lethality occurs prior to the onset of hematopoiesis in Fgfr1-deficient mice (Deng et al., 1994; Yamaguchi et al., 1994), the $F g f r 1^{-/}$mESCs failed to generate $\mathrm{Flk1}^{+}$mesoderm or BL-CFCs following differentiation in vitro. A role for Fgf signaling in early hematopoietic specification was further provided by studies showing that the addition of Fgf2 (basic Fgf; bFgf), a ligand of Fgfr1, at the onset of mESC differentiation led to a dose-dependent increase in the number of BL-CFCs (Faloon et al., 2000). The requirement for Fgf signaling is also implicated in later stages of hematopoietic development. The expression of an inducible dominant-negative $f g f r l$ in the zebrafish embryo after the emergence of primitive erythrocytes, led to a reduction in the number of definitive hematopoietic cells in the dorsal aorta (Lee et al., 2014; Pouget et al., 2014). In the human, FGF2 is essential for hPSC survival and proliferation leading to its inclusion in most hPSC medias (Greber et al., 2007; Vallier et al., 2005). In addition to its role in pluripotency, FGF2 has been shown to act in 
conjunction with BMP4 to induce mesoderm (Bernardo et al., 2011) and is required for BL-CFCderived blast colony growth (Kennedy et al., 2007; Vodyanik et al., 2010).

\subsubsection{Wnt signaling}

Wnts are a family of secreted proteins that are required in development and also has broad functional roles in the adult. There are 19 different Wnt proteins in both the mouse and human that are grouped by sequence similarity. Following their translation, Wnt proteins are modified by palmitoylation and glycosylation prior to their secretion. In the absence of Wnt, the $\beta$-catenin transcription factor is complexed with Axin, Apc and the serine-threonine kinases, Ck1a and Gsk3. The kinases in this complex phosphorylate $\beta$-catenin leading to its ubiquitination and degradation by the proteasome. In active signaling, the Wnt co-receptor (Lrp5/6) and a Frizzled protein form a complex to bind Wnt. This leads to the recruitment of Disheveled and the Axin/Apc/Ck1a/Gsk3 complex to the cell membrane. $\beta$-catenin then accumulates in the cytoplasm and enters the nucleus to regulate the transcription of target genes in conjunction with Tcf/Lef. Wnt signaling is tightly regulated and this pathway is antagonized by the expression of secreted Frizzled proteins and Wnt inhibitory protein (Wifl), which prevent ligand binding to cells, and Dickkopf proteins that negatively regulate signaling through their binding to Lrp5/6 (reviewed in Clevers and Nusse, 2012; Steinhart and Angers, 2018)

Evidence for an early developmental role for Wnt signaling was provided by McMahon and Moon (1989), who showed that the injection of Wnt1 (int-1) into early-staged Xenopus laevis embryos led to the duplication of the anterior-posterior body axis. In the mouse, Wnt3 is expressed in the epiblast of the E6.25 embryo, as well as in the primitive streak and mesoderm at later developmental stages. Genetic ablation of Wnt3 resulted in embryos that fail to generate a primitive streak (Liu et al., 1999) indicating that Wnt signaling is essential for gastrulation. Studies using the mESC model confirmed an early role for Wnt signaling, as the addition of Wnt3a was found to induce Brachyury expression, whereas early inhibition blocked its development (Gadue et al., 2006). Beyond primitive streak formation, Wnt signaling was also shown to play a role at subsequent stages of development in the mESC system, including the induction of $\mathrm{Flk}^{+}$ hematopoietic mesoderm from the primitive streak and the specification of primitive, but not definitive hematopoietic progenitors from mesoderm (Lengerke et al., 2008; Nostro et al., 2008). A role for Wnt signaling has also been defined in the EMP program through the demonstration 
that the addition of Wnt3a to explant cultures of the mouse E8.5 yolk sac increases colony-forming progenitor number. However, no benefit was seen when it was applied to cultures of sorted E9.5 $\mathrm{CD} 41^{+} \mathrm{Kit}^{+} \mathrm{CD} 16 / 32^{+}$EMPs. The demonstration that EMP colony-forming activity is reduced in mice that lack $\beta$-catenin in Cdh5-expressing cells (Frame et al., 2016) indicates that Wnt either directly induces EHT of EMP HECs or that it acts indirectly through other yolk sac cells. Taken together, these studies define a role for Wnt signaling at several stages of hematopoietic development.

As is the case in the mouse, studies using hPSCs have demonstrated that Wnt is essential to induce a primitive streak (Martyn et al., 2018). hPSC differentiation studies from our group have shown that Wnt signaling differentially regulates the induction of mesoderm fated to the primitive or definitive hematopoietic programs (Sturgeon et al., 2014).

\subsubsection{Vascular endothelial growth factor signaling}

Vascular endothelial growth factors (Vegfs) are important regulators of developmental processes, including vasculogenesis. Vegfs (Vegfa, Vegfb and Vegfc) signal through receptor tyrosine kinases: Vegfr1, Vegfr2 and Vegfr3. Vegfa predominantly binds to Vegfr2, an interaction that leads to the phosphorylation of the kinase domain of the receptor and the subsequent phosphorylation of downstream targets. Vegfa can also bind Vegfr1, however this binding interaction acts to limit the availability of the ligand for Vegfr2 thereby negatively regulating signaling. Vegfb can also bind Vegfr1, whereas Vegfc interacts with Vegfr3. Like Fgfs, Vegf/Vegfr ligand-receptor interactions are stabilized by HPSGs and other proteins, including Nrp1. The specificity of these ligand receptor interactions is conferred through alternative splicing and post-translational modifications (reviewed in Karaman et al., 2018).

In the early embryo, Flk1 (Vegfr1; Kdr) is expressed broadly in lateral plate mesoderm, as it is specified from the primitive streak (Yamaguchi et al., 1993). Flk1 is a defining marker of the mesoderm that contains BL-CFCs and its expression persists in the endothelial lineage, but is downregulated upon specification to the hematopoietic fates (Faloon et al., 2000; Fehling et al., 2003; Kabrun et al., 1997). Lineage tracing experiments have demonstrated that both the primitive and definitive hematopoietic programs derive from Flkl-expressing progenitors (Lugus et al., 2009), a finding consistent with the observation that the receptor is expressed on mesoderm. A role for this pathway in hematopoietic development in vivo was provided by gene targeting studies that 
showed that both Flk1 and Vegf are required for the generation of the hematopoietic and endothelial lineages (Carmeliet et al., 1996; Ferrara et al., 1996; Shalaby et al., 1997; Shalaby et al., 1995). Studies in the mouse and human PSC differentiation systems have shown that Vegf/Flk1 (human: VEGF/KDR) signaling is required to induce primitive and definitive hematopoiesis from mesoderm (Kennedy et al., 2007; Kennedy et al., 1997; Pearson et al., 2008; Pick et al., 2007).

\subsubsection{Notch signaling}

Notch signaling regulates the development of multiple lineages during embryogenesis and tissue homeostasis in the adult. The Notch ligands, Delta-like and Jagged proteins (D11, D13, D14, Jag1 and Jag2) signal through Notch receptors (Notch1, Notch2, Notch3 and Notch4). The interaction between the Notch ligands and receptors initiates two proteolytic cleavage events. In the first, the extracellular domain of the Notch receptor is cleaved by ADAM proteases and in the second, the intracellular domain is cleaved by the $\gamma$-secretase complex. The freed Notch intracellular domain (NICD) enters the nucleus and interacts with the downstream targets, Cbf1 (Rbpj- $\kappa)$ and Mam to regulate the expression of target genes (reviewed in Bray, 2016; Butko et al., 2016).

A role for Notch signaling in early development is provided by the finding that Notch1 and Notch2 are expressed as early as E7.5 in the posterior primitive streak and mesoderm (Del Amo et al., 1992; Reaume et al., 1992; Weinmaster et al., 1992). The expression of Notch1 in intra-aortic clusters and aortic endothelial cells of the E11.5 AGM raised the possibility that Notch regulates the emergence of the definitive hematopoietic program (Kumano et al., 2003; Villa et al., 2001). Genetic ablation of Notch1 results in embryonic lethality by E11.5 due to substantial cell death in the nervous system and abnormal somitogenesis (Conlon et al., 1995; Swiatek et al., 1994). However, the overlapping expression pattern of Notch1 and Notch2 raised the possibility of genetic compensation and that Notch signaling is active and required earlier in development. This was confirmed by Oka et al. (1995) that observed severe developmental defects at E8.5 in mice deficient for the Notch transcription factor, Cbfl. Evidence for a specific role for Notch signaling in definitive hematopoietic development was provided by studies that showed that colony-forming potential of E9.5 Notch1/- P-Sp, but not yolk sac explant cultures was abrogated in Notch1/mutants (Kumano et al., 2003). The findings from studies in the early embryo were consistent with the above in vitro analyses and showed that the number of hematopoietic colony-forming progenitors in the yolk sac between E7.5 and E8.5 was unaffected in the absence of Notch1 
(Hadland et al., 2004). To determine if the deficiency in definitive hematopoiesis was cell autonomous, Hadland et al. (2004) generated chimeric mice by injecting Notch1 ${ }^{-/-} ; \mathrm{ROSA}_{2} 6^{\mathrm{LacZ}}$ mESCs into wild type host blastocysts. Consistent with the studies described above, Notch1deficient cells contributed to yolk sac hematopoiesis between E9.5 and E10.5. However, the contribution of Notch1-null cells to hematopoietic progenitors in the yolk sac between E11.5 and 12.5, and the liver between E13.5 and E14.5 was reduced and completely absent in the post-natal bone marrow, respectively. These findings demonstrated a cell-autonomous requirement for Notch1 in the definitive hematopoietic program and raised the possibility that Notch1 is required at later stages of development in the EMP program. Evidence that Notch signaling plays a role in EHT has been provided by studies that showed that treating the HEC-containing $\left(\mathrm{CD} 31^{+} \mathrm{CD} 45^{-}\right.$ Ter119-) population isolated from the E11.5 AGM with anti-Jag1 blocking antibodies or the Notch inhibitor, DAPT abrogates the generation of $\mathrm{CD} 45^{+}$cells (Gama-Norton et al., 2015; Souilhol et al., 2016). Evidence has also been provided that the strength of Notch signaling is an important regulator of EHT. Through co-cultures with engineered OP9 stromal cells Gama-Norton et al. (2015) showed that low Notch signaling induced by the culture of the CD $31^{+} \mathrm{CD} 45^{-}$Ter $119^{-}$ population isolated from the E11.5 AGM with OP9-Jag1 cells efficiently produced CD45 ${ }^{+}$cells, whereas culture with OP9-D14 cells, which strongly activates Notch signaling, generated significantly fewer CD $45^{+}$cells. Beyond EHT, Notch signaling like plays additional roles in the expansion of early HSCs, as Hadland et al. (2015) showed that the culture of CD $144^{+} \mathrm{CD} 45^{+}$cells isolated from the E11.0 AGM with plate-bound D11 improved engraftment in adult mice. Taken together, the above studies indicate that Notch signaling plays a role in definitive hematopoietic development and that different Notch ligands may function at different stages.

The importance of Notch signaling in hematopoietic development of model organisms has prompted studies to investigate the regulation of $\mathrm{NOTCH}$ in human hematopoietic development. Our group and others have shown that human definitive HECs require NOTCH signaling to undergo EHT to generate hematopoietic cells (Ditadi et al., 2015; Uenishi et al., 2018). It remains to be seen whether stage-specific Notch ligands are required for the development of the human definitive hematopoietic program, as seems to be the case in the mouse. 


\subsubsection{Retinoic acid signaling}

Retinoic acid (RA) signaling is a critical regulator of numerous development processes. RA is synthesized from retinol $(\mathrm{ROH}$; vitamin $\mathrm{A})$ that accumulates from dietary sources. $\mathrm{ROH}$ is disseminated in the circulation, in conjunction with retinol binding proteins and enters the cell in a complex through the Stra6 receptor. Thereafter, $\mathrm{ROH}$ is converted to RA through a series of oxidation reactions. In the first reaction, retinal dehydrogenases catalyze the conversion of $\mathrm{ROH}$ to retinal (RAL). Second, retinaldehyde dehydrogenases catalyze the conversion of RAL to alltrans RA (ATRA). ATRA mediates transcription through its interaction with retinoic acid and retinoid $\mathrm{X}$ receptors in the nucleus. The concentration of ATRA is highly controlled through the expression and activity of cytochrome P450 26 subfamily proteins that convert ATRA to inactive polar metabolites. The conversion to polar metabolites is an important step to limit the ability of the ATRA to diffuse across neighbouring cell membranes and activate downstream signaling (reviewed in Ghyselinck and Duester, 2019; Rhinn and Dolle, 2012).

There are 3 retinaldehyde dehydrogenases, Aldh1a1 (Raldh1), Aldh1a2 (Raldh2) and Aldh1a3 (Raldh3) that are expressed at different sites and stages during gestation and in adult life. Aldh1a2 is expressed in mesoderm from E7.5 and required at early stages of development (Niederreither et al., 1997; Niederreither et al., 1999). The early expression of Aldhla2 prompted a number of investigators to study the role of RA signaling in hematopoietic development. Studies in the zebrafish demonstrated that the addition of exogenous RA reduced the expression of the hematopoietic marker, gatal in a dose-dependent manner at a time when the hematopoietic cells of the zebrafish primitive hematopoietic program are generated. Complimentary studies have investigated the role of RA signaling in the development of mouse primitive hematopoietic program. Consistent with the above zebrafish studies, the number of primitive erythroid colonyforming progenitors decreased in a dose-dependent manner upon exposure of E7.5 yolk sac explants to RA. In contrast, treatment of the mouse yolk sac explants with an inhibitor of RA signaling, DEAB led to an increase in primitive colony-forming progenitor numbers (de Jong et al., 2010). These studies are supported by the observation that the development of the primitive erythroid lineage is unaffected in the E8.0 Raldh2-deficient yolk sac (Goldie et al., 2008). Taken together, these findings demonstrate that RA signaling is not required for primitive hematopoiesis and that this program is sensitive to the inappropriate activation of this pathway. 
A clear role for RA signaling in HSC development was established through the demonstration that the deletion of Raldh2 in Cdh5-expressing cells extinguished repopulating potential in the E11.5 embryo (Chanda et al., 2013). Analyses using the Aldefluor assay, which measures the activity of all aldehyde dehydrogenases (ALDHs) (Storms et al., 1999), showed that all repopulating activity in the $\mathrm{E} 11.5 \mathrm{AGM}$ is contained within the $\mathrm{AA} 4.1 / \mathrm{CD} 144^{+} \mathrm{CD} 45^{+}$hematopoietic population that is also $\mathrm{ALDH}^{+}$. Furthermore, the addition of ATRA to cultures generated from the E10.5 and E11.5 AGM improved engraftment potential (Chanda et al., 2013). In the human, ALDH activity can be used to identify HSC-containing populations in cord blood and adult mobilized peripheral blood (Fallon et al., 2003; Hess et al., 2004) indicative of a long-standing regulatory role in HSC function beyond embryogenesis.

Given its role in hematopoietic development in model organisms and function in human HSCs, multiple investigators have studied the effect of RA in hematopoietic differentiation using hPSCs. As is the case in the mouse, $A L D H 1 A 2$ is expressed at early stages of development, appearing soon after the induction of $B R A C Y U R Y$ (Yu et al., 2010). The inclusion of ATRA to cultures at later stages of hPSC differentiation that contained hematopoietic progenitors favoured myelopoiesis at the expense of erythropoiesis (Rönn et al., 2015; Yu et al., 2010). However, it is unclear which hematopoietic programs were contained in these cultures, as the end-stage populations were not thoroughly characterized.

\subsubsection{Transcription factor regulation of embryonic hematopoiesis}

In addition to the signaling pathways that govern embryonic hematopoietic development, a role for several transcription factors has also been described. Here, I review the role of three transcription factors that are implicated in early lineage decisions to specify the hematopoietic fates and are regulated in a program-specific manner.

\subsubsection{Scl}

Scl (Tal1; Lyl1) is a basic helix-loop-helix protein (Mellentin et al., 1989) that regulates the development of the hematopoietic system at multiple levels. In addition to its expression in mouse and human hematopoietic progenitors (Mouthon et al., 1993), Scl is expressed from E7.5 in the yolk sac and embryo of mice (Kallianpur et al., 1994). Genetic ablation of Scl leads to a failure of blood island development and is embryonic lethal at E9.5. In addition, these animals also lack 
hematopoietic colony-forming progenitors (Robb et al., 1995; Shivdasani et al., 1995) indicating that $\mathrm{Scl}$ is required for both primitive and EMP hematopoiesis. Analyses of chimeras generated with Scl-deficient mESCs revealed a cell autonomous requirement for $\mathrm{Scl}$ in the definitive hematopoietic program, as Scl-deficient cells failed to contribute to the erythroid, myeloid and lymphoid lineages in the bone marrow and peripheral blood of adult mice (Porcher et al., 1996; Robb et al., 1996). To further investigate the role of Scl in definitive hematopoietic development and HSC function, Mikkola et al. (2003b) established an inducible system to delete Scl at different developmental stages. While Scl is required for the development of the primitive, EMP and definitive hematopoietic programs, the conditional deletion of $\mathrm{Scl}$ in adult HSCs did not disrupt their function demonstrating a stage-specific role for this transcription factor in specifying the hematopoietic fates during embryonic development.

Expression analyses of differentiated populations generated from mESCs showed that Scl expression follows the upregulation of Flk1 (Chung et al., 2002). Although the majority of BLCFCs do not express Scl, it is upregulated at early stages of BL-CFC-derived blast colony development. Analyses of Scl-deficient cells revealed that the BL-CFCs could initiate blast colony development, but could not generate hematopoietic progeny demonstrating a role in the specification of the hematopoietic fates from mesoderm (D'Souza et al., 2005). Interestingly, explant cultures of E9.25 yolk sacs that lack Scl give rise to colonies of beating cardiomyocytes (Van Handel et al., 2012) suggestive of a broader role for Scl in regulating mesoderm lineage decisions between the hematopoietic and cardiac fates.

\subsubsection{Runx1}

Runx1 (Am11; Cebpa2) is part of the Runx family of transcription factors and regulates gene transcription through its interaction with the transcription factor, Cbf $\beta$. Runx 1 expression is detected in the blood islands of the E7.5 yolk sac and later in the embryonic vasculature from E8.5 (Lacaud et al., 2002; North et al., 1999; Samokhvalov et al., 2007). Runx1 is also expressed in the HECs that line the ventral wall of the dorsal aorta and in HSCs (North et al., 1999; North et al., 2002). Genetic ablation of Runx1 leads to embryonic lethality between E11.5 and E12.5 with a complete loss of hematopoietic colony-forming potential in yolk sac and liver of E10.5 and E11.5 mice (Okuda et al., 1996; Wang et al., 1996a). Analyses of colony-forming potential in the E8.09.0 yolk sac revealed that Runx1 is not required for the specification of the primitive erythroid 
lineage. In contrast, macrophage and EMP erythroid progenitor potential was lost in the absence of Runx1 (Lacaud et al., 2002). Consistent with a requirement in the generation of the EMP program, $\mathrm{CD} 41^{+} \mathrm{Kit}^{+} \mathrm{CD} 16 / 32^{+}$progenitors were also absent in the E10.5 yolk sac of Runx1deficient embryos (Frame et al., 2016). Taken together, these studies show that Runx1 is required for EMP, but not primitive hematopoiesis.

The absence of hematopoietic clusters in the aorta of E10.5 Runx1-deficient mice (North et al., 1999) and the inability of Runx1-deficient cells to contribute to the hematopoietic populations in the thymus, spleen and peripheral blood of adult chimeric mice (Okuda et al., 1996) indicated the involvement of Runx1 in the generation of the definitive hematopoietic program. The stagespecific requirement for Runx1 in generation of the definitive hematopoietic program was mapped through its conditional deletion in cells that express Cdh5 or Vav. Their expression marks HECs and their hematopoietic progeny, respectively (Bustelo et al., 1993; Ogilvy et al., 1998; Ogilvy et al., 1999). Deletion of both alleles of Runx1 using Cdh5 led to a loss of expression in HECs and a failure to generate HSCs. In contrast, HSCs were generated when Runx1 was deleted in Vavexpressing cells (Chen et al., 2009) demonstrating a pivotal role for Runx1 in the formation, but not the function of HSCs.

\subsubsection{Myb}

Myb is an important regulator of embryonic and adult hematopoiesis through its interaction with the transcription factors, Cbp and p300 (Dai et al., 1996; Oelgeschlager et al., 1996). Myb is not expressed in the yolk sac at E8.5, but is later detected in the liver at E12.5 (Tober et al., 2008). Genetic ablation of Myb leads to sudden anemia at E14.0 and embryonic lethality at E15.0 (Mucenski et al., 1991). Analyses of the hematopoietic colony-forming progenitor potential of the E7.0-E9.0 yolk sac revealed that the primitive erythroid, macrophage and megakaryocytic lineages develop in the absence of Myb (Tober et al., 2008). In addition, tissue-resident macrophages develop normally in the brain, liver, skin, spleen, pancreas, kidney and lung of Myb-deficient mice (Schulz et al., 2012). In contrast, the generation of EMP erythroid lineage is lost in the absence of Myb (Tober et al., 2008). Analyses of chimeras generated through the injection of $\mathrm{Myb}^{-/-} \mathrm{mESCs}$ into immunodeficient Rag1 ${ }^{-/-}$blastocysts (Mombaerts et al., 1992), showed that the Myb-null cells do not contribute to any of the lymphoid lineages (Allen et al., 1999) indicating its essential role in the development of the LMP and definitive hematopoietic programs. Further evidence of its 
requirement for the generation of the definitive hematopoietic program came from analyses of explant cultures of the E9.5 and E11.5 $\mathrm{Myb}^{-/}$AGM, which showed a complete lack hematopoietic colony-forming progenitor activity (Mukouyama et al., 1999).

\subsection{Modeling human embryonic hematopoietic development}

Analyses of model organism development has led to remarkable improvements in our understanding of the genesis of the different embryonic hematopoietic programs. However, insights into how the human embryonic hematopoietic system develops has proved challenging. The characterization of the hematopoietic programs in human embryos have largely been limited to morphologic and transcriptomic analyses given the limited availability of tissue at the relevant gestational ages. Given the challenges of accessing human embryonic tissue, the field has turned to the hPSC differentiation system to model and study early human embryonic development.

\subsubsection{Hematopoietic development using human pluripotent stem cells}

hPSC differentiation to the hematopoietic fates was pioneered using serum-based stromal cocultures (Kaufman et al., 2001; Vodyanik et al., 2005). Studies using this culture format demonstrated that hPSCs could differentiate to cells of the erythroid, myeloid and lymphoid lineages and that their development could be monitored by the onset of CD43 (Sialophorin; SPN) expression (Galic et al., 2006; Kaufman et al., 2001; Vodyanik et al., 2005). This co-culture approach was soon abandoned, as the presence of stromal cells and serum in the media made it difficult to identify the signaling pathways that regulate hematopoietic specification from hPSCs.

Thereafter, hPSC differentiation protocols implemented directed differentiation strategies that were informed by our understanding of developmental hematopoiesis in model organisms (reviewed in Ditadi et al., 2017; Ivanovs et al., 2017). Early directed differentiation protocols largely generated the primitive hematopoietic program marked by the rapid induction of erythroid progenitors that gave rise to small colonies of erythroblasts that expressed high levels of the embryonic $\beta$ globin, $H B E$ and low levels of fetal $\beta$ globin, $H B G$. Analyses of the stages preceding the onset of the primitive hematopoietic program demonstrated the emergence of $\mathrm{KDR}^{+} \mathrm{BL}-\mathrm{CFCs}$ that generated blast colonies with hematopoietic and endothelial potential, similar to those characterized in the mESC model and mouse embryo. These observations indicated that the onset of embryonic hematopoiesis in the human, as is the case in the mouse, is defined by the 
development of the hemangioblast (Kennedy et al., 2007). A contemporaneous study demonstrated that the primitive hematopoietic program was followed by a second wave of hematopoietic progenitors that gave rise to erythroid colonies that expressed embryonic $(\mathrm{HbE})$ and fetal $(\mathrm{HbF}) \beta$ globin (Zambidis et al., 2008) suggestive of the emergence of the EMP program. Analyses of populations from later stages of hPSC differentiation cultures led to the identification of progenitors that displayed characteristics of HECs. These progenitors expressed CD144, CD31, KDR, KIT and CD34, but not CD43 or CD45, and when cultured under the appropriate conditions, could generate erythroid, myeloid and lymphoid progeny (Choi et al., 2012; Kennedy et al., 2012). The demonstration that $\mathrm{CD} 34^{+} \mathrm{CD}^{-} 3^{-}$progenitors with $\mathrm{T}$ lymphoid potential developed following the $\mathrm{CD} 43^{+}$primitive hematopoietic wave strongly suggested that these later emerging progenitors represented either the LMP or definitive hematopoietic programs. The presence of the primitive, EMP and LMP/definitive derivatives in the same cultures made it difficult to determine what programs were induced. To address this, our group investigated the regulation of these different programs at mesoderm induction stage and found that the inhibition of TGF $\beta$ signaling with SB431542 at day 1.75 of differentiation blocked the emergence of $\mathrm{CD} 43^{+}$primitive hematopoietic cells and preserved progenitors with $\mathrm{T}$ lymphoid potential in the $\mathrm{CD} 34^{+} \mathrm{CD} 43^{-}$population at day 9 of differentiation (Kennedy et al., 2012). The differential sensitivity of the primitive hematopoietic and T lymphoid fates to TGF $\beta$ inhibition at this early stage of hPSC differentiation culture raised the possibility that these lineages emerge from distinct populations of mesoderm. To investigate the heterogeneity of the $\mathrm{KDR}^{+}$mesoderm population, our lab performed an anti-CD antibody library screen with the Princess Margaret Genomics Centre and found that that CD235a (Glycophorin A; GYPA) is expressed on a subset of these cells. Further analyses showed that the expression of CD235a segregated the primitive hematopoietic and $\mathrm{T}$ lymphoid fates, whereby $\mathrm{KDR}^{+} \mathrm{CD} 235 \mathrm{a}^{+}$mesoderm gave rise to the hematopoietic progenitors of the primitive hematopoietic program, while $\mathrm{KDR}^{+} \mathrm{CD} 235 \mathrm{a}^{-}$mesoderm gave rise to $\mathrm{T}$ lymphoid progenitors thought to be representative of the definitive hematopoietic program. Further investigation of the regulation of these two subsets of $\mathrm{KDR}^{+}$mesoderm demonstrated that Wnt signaling is also an important regulator of these populations. The activation of Wnt signaling with the GSK3 $\beta$ inhibitor, CHIR99021 (at day 1.75 of differentiation) promoted the development of mesoderm that gave rise to erythroid, myeloid and $\mathrm{T}$ lymphoid, but not the primitive hematopoietic progeny. In contrast, the inhibition of Wnt signaling using the PORCN inhibitor, IWP2 generated mesoderm with primitive hematopoietic potential that failed to give rise to $\mathrm{T}$ lymphoid progenitors. In 
addition to the primitive hematopoietic program, IWP2-induced cultures also contained progenitors that gave rise to colonies of EMP-like erythroblasts that expressed the embryonic $H B E$ and fetal $H B G \beta$ globin. Although devoid of T lymphoid potential, the $\mathrm{CD} 34^{+} \mathrm{CD}^{-} 3^{-}$population in IWP2-treated cultures also gave rise to the NK cell lineage (Sturgeon et al., 2014). Characterization of these NK cells showed that they share transcriptomic and functional features of NK cells derived from the $\mathrm{CD} 41^{+} \mathrm{Kit}^{+} \mathrm{CD} 16 / 32^{+}$EMP population in the E9.5 mouse yolk sac. By comparison, the NK cells generated from cultures induced with CHIR99021 more closely resembled those differentiated from adult mouse and CD $34^{+}$human cord blood progenitors (Dege et al., 2020).

The ability to specify either fate at the mesoderm level enabled the generation of populations enriched in the progenitors of either the primitive or definitive hematopoietic programs. Access to the definitive-enriched populations allowed for detailed studies on the specification and development of definitive HECs that provided the following new insights. First, the findings showed that HECs underwent EHT to generate hematopoietic progeny in a NOTCH-dependent manner. Second, clonal analyses showed that single HECs are multipotent and give rise to the erythroid, myeloid and T lymphoid lineages. Third, the CD $34^{+} \mathrm{CD} 43^{-}$subpopulation that contained these HECs either gave rise to hematopoietic progeny or vascular progeny, but not both indicating that the HECs and endothelial progenitors represent distinct cell types (Ditadi et al., 2015). Molecular analyses of the populations generated from SB431542/CHIR99021-induced mesoderm provided further evidence that they represented the definitive hematopoietic program. These studies showed that CD34 ${ }^{+} \mathrm{CD} 43^{-}$populations generated from SB-431542/CHIR99021-induced mesoderm express higher levels of $\mathrm{HOXA}$ genes than $\mathrm{CD} 34^{+} \mathrm{CD} 43^{-}$populations generated from IWP2 or Activin A-induced primitive mesoderm (Dege et al., 2020; Ng et al., 2016). Additionally, $\mathrm{Ng}$ et al. (2016) demonstrated that cells from SB431542/CHIR99021-induced cultures displayed expression patterns of $H O X A$ genes and regulators of retinoic acid signaling similar to those found in human AGM populations. The expression of $H O X A$ genes is likely critical to the successful generation of HSCs from hPSCs, as loss-of-function studies have demonstrated that HOXA5 and HOXA7 are required for the engraftment of human fetal liver HSCs in immunodeficient mice (Dou et al., 2016). Despite these findings, the progenitor populations generated in these studies have failed to engraft immunodeficient mice. To date, hPSC-derived progenitors with engraftment potential have only been generated through the formation of $\mathrm{CD}_{4} 5^{+}$hematopoietic populations in teratomas or the enforced expression of transcription factors in definitive HECs (Amabile et al., 
2013; Sugimura et al., 2017; Suzuki et al., 2013). The failure to generate HSCs from hPSCs in the absence of teratomas or enforced transcription factor expression indicates that the hPSC differentiation protocols are missing additional essential signaling or environment factors required for their specification. 


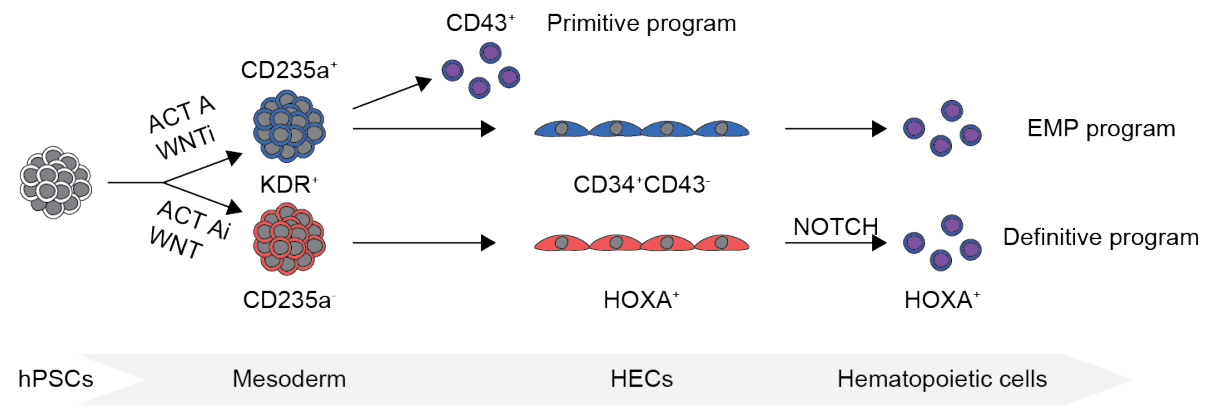

Figure 1.4 Model of human hematopoietic development using pluripotent stem cells.

Analyses of hematopoietic differentiation using hPSCs have revealed that primitive and EMP programs originate from $\mathrm{KDR}^{+} \mathrm{CD} 235 \mathrm{a}^{+}$mesoderm and is induced in cultures that contain Activin A (ACT A) or WNT inhibitors (WNTi). In contrast, the definitive hematopoietic program emerges from $\mathrm{KDR}^{+} \mathrm{CD} 235 \mathrm{a}^{-}$mesoderm in cultures that contained WNT agonists and ACT A antagonists (ACT Ai). These cultures give rise to multipotent $\mathrm{HOXA}^{+}$HECs that undergo a NOTCHdependent endothelial-to-hematopoietic transition to generate hematopoietic cells. 


\subsection{Applications of human pluripotent stem cell-derived hematopoietic cells}

The generation of different hematopoietic cells from hPSCs facilitates access to hematopoietic progenitors that are only present during embryogenesis, such as those that give rise to tissueresident macrophages. These progenitor and end-stage populations can be differentiated in large numbers for downstream analyses or transplantation given the extensive proliferative ability of hPSCs. Genome engineering has enabled the generation of customized hPSC lines that may better recapitulate disease states or confer specialized cellular functions. In addition, the differentiation of induced PSCs (iPSCs) offers the opportunity to generate hematopoietic cells from any person.

\subsubsection{Modeling hematologic disease}

A number of investigators have modeled hematologic diseases using patient-derived iPSCs. However, as many of these diseases occur in adulthood, it is unclear if it is possible to accurately model them using hPSC-derived hematopoietic differentiation protocols that do not fully recapitulate the definitive hematopoietic program. Hematological diseases that occur early in life may be more amenable to modeling in vitro. For example, 10-15\% of individuals with Down syndrome are born with a transient myeloproliferative disorder characterized by the presence of immature megakaryocytes (megakaryoblasts) in the peripheral blood. Although this process often resolves, $20-30 \%$ of these individuals develop an acute leukemia later in life (reviewed in Antonarakis, 2017). As this disorder initiates prior to birth, the progenitors of the EMP program may be responsible for the mass accumulation of megakaryoblasts. Findings from analyses of hematopoietic potential of iPSCs derived from individuals with trisomy 21 were consistent with this interpretation. These studies showed that differentiated trisomy $21 \mathrm{iPSCs}$ displayed enhanced primitive erythroid, later stage erythroid, myeloid and megakaryocytic potential compared to populations generated from wild type iPSCs (Chou et al., 2012; Maclean et al., 2012). The later stage erythroid progenitors gave rise to colonies of erythroblasts that expressed elevated levels fetal $\beta$ globin, $H B G$ and lower levels of embryonic $\beta$ globin, $H B E$ suggestive of the EMP program (Maclean et al., 2012). These studies show that the yolk sac hematopoietic programs are dysregulated in Down syndrome and indicate that it may be possible to use populations differentiated from hPSCs to better understand the contribution of the yolk sac hematopoietic programs to leukemogenesis. 
The derivation of iPSCs from acute myeloid leukemia (AML) blast cells has provided insight into the fitness of different leukemic cells in a single patient. The clonal derivation of iPSCs from AML blasts yielded several lines that were representative of the distinct leukemia subclones present in the patient. Hematopoietic differentiation of these iPSC lines revealed differences in proliferative potential and drug sensitivity between AML subclones (Chao et al., 2017). In addition to analyses using patient-derived iPSCs, leukemic mutations can be engineered in existing hPSC lines using CRISPR-Cas9. Analyses of hematopoietic differentiation of hPSCs that express an ETV6-RUNX1 fusion, which is common in pediatric B cell leukemia (Shurtleff et al., 1995), revealed impaired B lymphoid development (Boiers et al., 2018) indicating that embryonic B cell progenitors, including those generated from the LMP program may also be susceptible to malignant transformation, as blocked differentiation is a key step in this process.

In addition to leukemias, hematologic genetic diseases have also been studied using hPSCs. Attempts to model immunodeficiencies showed that IL2RG-deficient hPSCs do not differentiate to the NK cell and T lymphoid fates ( $\mathrm{Li}$ et al., 2016). RAG1-deficient iPSCs also failed to generate T lymphocytes following differentiation. In this case, the block in development appeared to be at the progenitor stage marked by the expression of CD5 and CD7 and lack of CD4 and CD8 (Brauer et al., 2016). Although these genetic requirements were previously demonstrated in human with severe combined immunodeficiency (Puck et al., 1993; Schwarz et al., 1996), these tools nonetheless provide a platform to better understand the stage at which lineage development is blocked and to develop novel strategies to treat these diseases (Alzubi et al., 2017).

Models of other hematologic diseases have been established, including those for ShwachmanDiamond and Diamond-Blackfan syndromes. Shwachman-Diamond syndrome results from mutations in the SBDS gene (Boocock et al., 2003) and is characterized by exocrine pancreatic insufficiency and bone marrow failure (Shwachman et al., 1964). The differentiation of SBDS $S^{-1}$ hESCs and patient-derived iPSCs recapitulated key aspects of the disease, including elevated protease activity in the pancreatic and hematopoietic lineages (Tulpule et al., 2013). Another disease, Diamond-Blackfan syndrome, which is caused by mutations in ribosomal genes (Draptchinskaia et al., 1999) and is characterized by a failure of erythropoiesis (Diamond and Blackfan, 1938) has also been modeled using patient-derived iPSCs. Differentiation of these iPSCs revealed that the cells displayed a deficiency in erythroid differentiation. To identify potential therapeutic agents that could overcome this block and promote erythroid differentiation, iPSC- 
derived erythroid progenitors were treated with 1280 small molecules contained in the LOPAC drug library and 160 other molecules. From this screen, SMER28, an inhibitor of autophagy was shown to rescue erythropoiesis (Doulatov et al., 2017). The erythroid progeny generated from these cultures predominantly expressed the fetal $\beta$ globin, $H B G$ suggesting that SMER28 is able to rescue fetal-like erythropoiesis. Rescue of erythroid lineage cells that express the adult $\beta$ globin, $H B B$ is challenging with differentiated patient-derived iPSCs, as it is not yet possible to generate these cells from hPSCs.

While hPSC differentiation provide a source of cells for intensive analyses, it also facilitates access to populations that are difficult to sample from humans. Analyses of iPSC-derived microglia, the tissue-resident macrophage of the brain, demonstrated that microglia from individuals with familial Mediterranean fever elicit elevated inflammatory responses upon stimulation compared to iPSC-derived microglia from a healthy donor (Takata et al., 2017). In addition, the transplantation of hPSC-derived microglia into the mouse brain has been used to study their role Alzheimer's Disease following intracranial injection of amyloid- $\beta$ or using transgenic mice engineered to express it (Abud et al., 2017; Hasselmann et al., 2019; Mancuso et al., 2019).

\subsubsection{Development of novel therapies}

Chimeric antigen receptor (CAR) $\mathrm{T}$ and NK cells have emerged as potent anti-cancer therapies (Brown et al., 2016; Liu et al., 2020; Maude et al., 2018; Park et al., 2018). The demonstration that hPSC-derived CAR T and NK cells showed anti-tumor activity in mice (Themeli et al., 2013) raised the possibility that hPSCs could provide a rich source of allogenic cells for therapy. Indeed, clinical trials using hPSC-derived NK cells to treat patients with solid tumors (NCT03841110) and COVID19 (NCT04363346) were launched in 2019 and 2020, respectively. Trials with hPSCderived CAR-T cells will likely follow. Other hPSC-derived hematopoietic cells may also prove beneficial to treat cancers as demonstrated by the anti-tumor activity of CAR macrophages that were generated from peripheral blood monocytes (Klichinsky et al., 2020).

The transplantation of macrophages generated from peripheral blood monocytes is being explored as a treatment for liver cirrhosis (Moroni et al., 2019). Given that the yolk sac hematopoietic programs give rise to Kupffer cells, the tissue-resident macrophage of the liver, it is possible that hPSC-derived macrophages from the primitive and EMP programs offer additional therapeutic benefit. Similarly, the transplantation of the hPSC-derived lymphoid progenitors from the LMP 
program may replenish populations that were generated in embryonic development and have become depleted or dysregulated in disease.

\subsection{The goal of this project}

Despite significant advances in our understanding of human embryonic hematopoietic development, many questions remain surrounding the lineage relationships between the different developmental programs. This work builds upon previous studies from our group that demonstrated the mesoderm that gives rise to the primitive and definitive hematopoietic programs can be distinguished based on the expression of CD235a and that their specification is regulated by Activin A and Wnt signaling. Based on these findings, I hypothesized that $\mathrm{KDR}^{+} \mathrm{CD} 235 \mathrm{a}^{+}$ mesoderm is representative of yolk sac hematopoietic mesoderm and should give rise to all of the hematopoietic lineages generated from this tissue in vivo. In this thesis, I describe the cell signaling and transcription factor regulation of the human primitive hematopoietic program. In addition, I investigate the development of the EMP and LMP programs from hPSCs and the lineage relationship between them. Based on these analyses, I describe a system to track the lineage potential of single mesoderm cells to understand the clonal contribution to the yolk sac hematopoietic programs. Taken together, this thesis maps the architecture of the developing human

embryonic hematopoietic system and in doing so provides a protocol to generate hematopoietic populations that will be essential to realize the full therapeutic applications of hPSC-derived hematopoietic cells. 


\section{Chapter 2}

\section{Methods}

\subsection{Acknowledgements}

I thank Deborah French (Children's Hospital of Philadelphia) for the CHOP10WT iPSC line, Feng Zhang for the PX458 pSpCas9(BB)-2A-GFP plasmid (Addgene plasmid 48138), Nestor Fernandez for the CRISPR-Cas9 targeting strategy to knockout MYB from hPSCs, the SickKids/UHN Flow Cytometry Facility for their assistance with cell sorting, The Centre for Applied Genomics (TCAG) for Sanger sequencing, Cell Line Genetics for the karyotypic analysis and Shahin Rafii (Weill Cornell Medicine) for the HUVEC-E4ORF1 endothelial cell line.

\section{2 hPSC culture}

The H1 human embryonic stem cell line (hESC) (Thomson et al., 1998) and CHOPWT10 human iPSC line (Maguire et al., 2016) were used in this study. hPSCs were maintained on irradiated mouse embryonic fibroblasts (iMEFs) in human embryonic stem cell media containing DMEM/F12 (Cellgro) with penicillin/streptomycin (1\%, ThermoFisher), L-glutamine (2 mM, ThermoFisher), non-essential amino acids (1x, ThermoFisher), $\beta$-mercaptoethanol (55 $\mu \mathrm{M}$, ThermoFisher) and KnockOut serum replacement (20\%, ThermoFisher) on $0.1 \%$ gelatin (Millipore Sigma)-coated tissue culture plates. Media supplemented with FGF2 (H1: 15 ng/mL and CHOPWT10: $10 \mathrm{ng} / \mathrm{mL}, \mathrm{R} \& D$ Systems) was changed daily for 7 days prior to differentiation. hPSCs were routinely tested for mycoplasma. hPSCs were maintained in normoxic conditions $\left(37^{\circ} \mathrm{C}, 5 \% \mathrm{CO}_{2}\right)$.

\subsection{CRISPR-Cas9 genome editing in hPSCs}

The H1 hESC line was cultured as described in Section 2.2 for 2 days in penicillin/streptomycinfree hESC media prior to transfection. The sgRNA guide targeted to exon 5 of $M Y B$ was cloned into BbsI restriction site downstream of the U6 promoter in the PX458 pSpCas9(BB)-2A-GFP plasmid (Addgene plasmid 48138; Ran et al., 2013). The PX458 pSpCas9(MYB-exon5)-2A-GFP plasmid $(2 \mu \mathrm{g})$ and single-stranded oligonucleotide (ssODN; 2 fmol; Integrated DNA Technologies) were prepared in X-tremeGENE 9 DNA Transfection Reagent $(6 \mu \mathrm{L} / 100 \mu \mathrm{L}$, 
ROCHE) in DMEM/F12 and incubated for 30 minutes at room temperature (Table 2.1). Thereafter, the DNA complex was added dropwise to the hESC culture and incubated overnight under normoxic conditions $\left(37^{\circ} \mathrm{C}, 5 \% \mathrm{CO}_{2}\right)$.

Table 2.1 Sequence information for CRISPR-Cas9 genome editing.

\begin{tabular}{|c|c|}
\hline Reagent & Sequence (5'-3') \\
\hline$M Y B$ sgRNA & AGAAATACGGTCCGAAACGTTGG \\
\hline ssODN & $\begin{array}{l}\text { ATTACATAACTTTAAAACATAGGTTATTTTTGTGTGTTTATCTGAAGGTGATAG } \\
\text { AGCTTGTACAGAAATATAATAGTGATCTAGATTATTGCCAAGCACTTAAAGGGG } \\
\text { AGAATTGGAAAACAATGTAGGGAGAGGTGGCATAACCACTTGAATCC }\end{array}$ \\
\hline
\end{tabular}

After 16 hours, cultures were treated with TrypLE (ThermoFisher) for 3 minutes at $37^{\circ} \mathrm{C}$ to generate a single cell suspension. $\mathrm{eGFP}^{+}$cells were isolated by FACS and collected in hESC media supplemented with FGF2 (15 ng/mL) and ROCK inhibitor Y-27632 (10 $\mu \mathrm{M}$, TOCRIS). Cells were sorted through a $100 \mu \mathrm{m}$ nozzle using the Influx (BD) cell sorter at the SickKids/UHN Flow Cytometry Facility. After cell sorting, 10,000 $\mathrm{GFP}^{+}$cells were plated on iMEFs in hESC media supplemented with FGF2 (15 ng/mL) and ROCK inhibitor Y-27632 (10 $\mu \mathrm{M})$ on 0.1\% gelatincoated $10 \mathrm{~cm}$ tissue culture dishes. After 24 hours, the ROCK inhibitor Y-27632 was removed and the cultures were maintained as described in Section 2.2 for 10 days.

Following their expansion, individual hESC colonies were mechanically isolated and treated with TrypLE for 3 minutes at $37^{\circ} \mathrm{C}$ prior to transfer to hESC media supplemented with FGF2 (15 $\mathrm{ng} / \mathrm{mL}$ ) in individual wells of $0.1 \%$ gelatin-coated 96 -well tissue culture plates containing iMEFs. Colonies with differentiated cells or those in close proximity to other colonies were excluded. The cultures were maintained as described in Section 2.2 to expand clones for DNA screening and cryopreservation.

Following clonal expansion, a fraction of the clone was transferred to hESC media supplemented with FGF2 (15 ng/mL) on 25\% Matrigel (Corning)-coated plates to deplete the iMEFs. Thereafter, DNA was isolated using the DNeasy Blood and Tissue Kit (QIAGEN). The targeted locus was amplified by PCR (Table 2.2). PCR was performed on a MasterCycler EP Gradient 96-well Thermal Cycler (Eppendorf) with Biolase DNA Polymerase (Bioline). Because the insertion of the ssODN to $M Y B$ introduces a novel XbaI restriction site, $\mathrm{PCR}$ amplicons were treated with XbaI 
(New England Biolabs) prior to electrophoretic separation on an Agarose (1\%, FroggaBio) gel containing Ethidium Bromide (Millipore Sigma). The genotypes of XbaI homozygous clones were confirmed by Sanger sequencing.

Table 2.2 Primer sequences used to screen hESC clones.

\begin{tabular}{|l|l|}
\hline Primer & Sequence (5'-3') \\
\hline Forward & TTCTTTCTTCATTGGTGTGTG \\
\hline Reverse & CAGAATGTTCAATACCCACTC \\
\hline
\end{tabular}

Karyotypic analysis of one $\mathrm{MYB}^{-/}$hESC clone was performed (Cell Line Genetics). In all experiments that used $\mathrm{MYB}^{-/-} \mathrm{hESCs}$, an untargeted clone that was subcloned in unison with the $\mathrm{MYB}^{-/-} \mathrm{hESC}$ clone was used for comparison.

\subsection{Hematopoietic differentiation from hPSCs}

hPSC differentiation to the hematopoietic lineage was performed in StemPro-34 media supplemented with penicillin/streptomycin (1\%, ThermoFisher), L-glutamine (2 mM, ThermoFisher), ascorbic acid (50 $\mu \mathrm{g} / \mathrm{mL}$, Millipore Sigma), transferrin (150 $\mu \mathrm{g} / \mathrm{mL}, \mathrm{ROCHE})$, monothioglycerol (50 $\mu \mathrm{g} / \mathrm{mL}$, Millipore Sigma) and other stage-specific factors. Differentiation cultures were maintained in hypoxic conditions $\left(37^{\circ} \mathrm{C}, 5 \% \mathrm{CO}_{2}, 5 \% \mathrm{O}_{2}\right)$ unless otherwise indicated. On day 0, hPSC cultures at 80-90\% confluency were treated with TrypLE for 3 minutes at $37^{\circ} \mathrm{C}$. Thereafter, the $80-90 \%$ of the TrypLE was aspirated and the cultures were incubated at $37^{\circ} \mathrm{C}$ for an additional 2 minutes. Small clusters of hPSCs $(<5$ cells per cluster) were generated by gentle pipetting and transferred to $4 \mathrm{~mL}$ of StemPro-34 media (Gibco) containing ROCK inhibitor Y-27632 $(10 \mu \mathrm{M})$ and BMP4 $(1 \mathrm{ng} / \mathrm{mL})$ at 500,000 cells/mL. Embryoid bodies (EBs) were generated in $60 \mathrm{~mm}$ Petri dishes on an orbital shaker (H1: 70 RPM; CHOPWT10: 60 RPM) for 18 hours. On day 1 of differentiation, the EBs were collected by centrifugation at 40 RCF for 5 minutes and cultured in StemPro-34 media supplemented with BMP4 (10 ng/mL), FGF2 (5 $\mathrm{ng} / \mathrm{mL})$ and Activin A (H1: $6 \mathrm{ng} / \mathrm{mL}$; CHOPWT10: $2 \mathrm{ng} / \mathrm{mL})$. Cultures were maintained under static conditions in 5\% poly(2-hydroxyethyl methacrylate) (Millipore Sigma)-treated tissue culture plates for the duration of the differentiation. On day 4 of differentiation, the EBs were collected by centrifugation at $150 \mathrm{RCF}$ for 5 minutes and cultured in StemPro-34 media supplemented with FGF2 (5ng/mL), VEGF (15 ng/mL), IL6 (10 ng/mL) and IL11 (10 ng/mL). From day 6 of 
differentiation onward, cultures were maintained in StemPro-34 media containing FGF2 (5ng/mL), VEGF (15 ng/mL), IL6 (10 ng/mL) and IL11 (10 ng/mL), SCF (100 ng/mL), IGF1 (50 $\mathrm{ng} / \mathrm{mL})$ and EPO $(4 \mathrm{U} / \mathrm{mL})$. Thereafter, the media was replenished every 2 days for the duration of the differentiation. To induce the differentiation of the definitive fate, day 1 EBs were collected by centrifugation at $40 \mathrm{RCF}$ for 5 minutes and were cultured in StemPro-34 media supplemented with BMP4 (10 ng/mL), FGF2 (5 ng/mL). On day 2 of differentiation, the EBs were collected by centrifugation at 150 RCF for 5 minutes and cultured in StemPro-34 media supplemented BMP4 (10 ng/mL), FGF2 (5 ng/mL) and SB431542 (6 $\mu \mathrm{M}$, TOCRIS). On day 3 of differentiation the EBs were again collected by centrifugation at 150 RCF for 5 minutes and cultured in StemPro-34 media supplemented with FGF2 (5ng/mL), VEGF (15 ng/mL), IL6 (10 ng/mL) and IL11 (10 $\mathrm{ng} / \mathrm{mL}$ ). The cultures were maintained from day 6 of differentiation onward, as described above. All recombinant factors are human and were purchased from R\&D Systems. In the experiments that investigated the role of NOTCH signaling, DMSO or the gamma secretase inhibitor L-685,458 (10 $\mu \mathrm{M}, \mathrm{R} \& \mathrm{D}$ Systems) were included in the media.

\subsection{Flow cytometry and cell sorting}

EBs prior to day 9 of differentiation, monolayer cultures and cultures aggregated after cell sorting were dissociated with Trypsin (Corning) for 5 minutes at $37^{\circ} \mathrm{C}$. From day 9 of differentiation onward, EB cultures were dissociated with Trypsin for 5 minutes at $37^{\circ} \mathrm{C}$ prior to incubation in collagenase type II $\left(0.2 \%\right.$, Worthington) for 1 hour at $37^{\circ} \mathrm{C}$. Cells were stained at a concentration less than or equal to $5,000,000$ cells $/ \mathrm{mL}$ for 30 minutes at $4^{\circ} \mathrm{C}$ in the dark. For flow cytometry, cells were stained in IMDM (Gibco) supplemented with penicillin/streptomycin (1\%), FCS (2\%, Wisent) and DNaseI (Millipore Sigma). For fluorescence-activated cell sorting (FACS), cells were stained, sorted and collected in StemPro-34 media supplemented with penicillin/streptomycin (1\%), L-glutamine $(2 \mathrm{mM})$ and DNaseI. Cells were sorted through a $100 \mu \mathrm{m}$ nozzle using the Influx (BD), AriaII (BD), AriaIII (BD) and MoFlo (Beckman-Coulter) cell sorters at the SickKids/UHN Flow Cytometry Facility. Thereafter, the sorted cells were counted and resuspended at 250,000 cells/mL in StemPro-34 media supplemented with recombinant factors appropriate for the day of differentiation. To aggregate the sorted cells, $250 \mu \mathrm{L}$ of the single cell suspension was transferred to individual wells of 5\% poly(2-hydroxyethyl methacrylate)-treated 24-well tissue culture plates. $750 \mu \mathrm{L}$ of supplemented StemPro-34 media was added 1 day after sorting. To generate monolayer cultures, $80 \mu \mathrm{L}$ of the single cell suspension was spotted on 
individual wells of 25\% Matrigel-coated 12-well tissue culture plates in supplemented StemPro34 media. After 1 day, $1 \mathrm{~mL}$ of supplemented StemPro-34 media was added.

To isolate hematopoietic cells from OP9-DL4 co-cultures, human cells were isolated by Magnetic Activated Cell Sorting (MACS). The cultures were dissociated with Trypsin for 5 minutes at $37^{\circ} \mathrm{C}$ and stained with the Mouse Cell Depletion Cocktail (1:5, Miltenyi) at 100,000,000 cells/mL for 15 minutes at $4{ }^{\circ} \mathrm{C}$ in the dark. Following MACS on an MS Column (Miltenyi), the flow-through fraction was stained with fluorescent-conjugated antibodies in Calcium and Magnesium-free PBS (CellGro) supplemented with $2 \%$ FCS and DNaseI for 30 minutes at $4^{\circ} \mathrm{C}$ in the dark. Cells were sorted and collected in Calcium and Magnesium-free PBS supplemented with 2\% FCS and DNaseI or deposited into wells of 96-well tissue culture plates containing $150 \mu \mathrm{L}$ of supplemented culture media and stromal cells where indicated.

The following antibodies were used: KDR-PE (3:20, clone 89106, R\&D Systems), KDR-Biotin (1:10, clone 89106, Novus Biologicals) CD235a/b-APC (1:100, clone HIR2/GA-R2, BD Pharmingen), CD34-PE-Cy7 (1:100, clone 4H11, ThermoFisher), CD34-APC (1:100, clone 8G12, BD Pharmingen), CD34-FITC (1:100, clone 8G12, BD Pharmingen), CD43-PE (3:100, clone 1G10, BD Pharmingen), CD43-FITC (1:10, clone 1G10, BD Pharmingen), CD43-APC-H7 (1:100, clone 1G10, BD Pharmingen), CD45-eFluor450 (1:50, clone HI30, ThermoFisher), CD45-APCCy7 (3:100, clone 2D1, BD Pharmingen), CD45-BV605 (1:50, clone HI30, BioLegend), CD117APC (1:50, clone 104D2, BD Pharmingen), CD117-PE (1:50, clone 104D2, BD Pharmingen), CD144-APC (3:100, clone BV9, BioLegend), CD31-FITC (3:20, clone WM59, BD Pharmingen), CD31-PE (3:100, clone WM59, BD Pharmingen), CD90-APC (1:1000, clone 5E10, BD Pharmingen), CD7-PE (7:100, clone M-T701, BD Pharmingen), CD7-APC (3:100, clone 1241D1, ThermoFisher), CD56-eFluor450 (1:50, clone TULY56, ThermoFisher), CD56-APC (3:100, clone B159, BD Pharmingen), CD5-FITC (1:10, clone UCHT2, BD Pharmingen), CD4-PE-Cy7 (1:50, clone SK3, ThermoFisher), CD8-PE (1:20, clone HIT8a, BD Pharmingen), CD3-FITC (1:50, clone UCHT1, ThermoFisher), TCR $\alpha \beta$-APC (3:100, clone IP26, BioLegend), TCR $\gamma \delta$-PE (5:100, clone IMMU510, Beckman Coulter), TCR $\gamma \delta$-BV421 (2:100, clone B1, BioLegend), CD19-PE-Cy7 (1:50, SJ25C1, BD Pharmingen), CD11b-APC (1:50, clone VIM12, Life Technologies), SSEA-5-APC (1:200, clone 8e11, BioLegend), TRA1-60-AF488 (3:100, clone TRA-1-60, BD Pharmingen), E-cadherin-PE (1:100, clone 67A4, BD Pharmingen) and CXCR4- 
BV421 (2:100, clone 12G5, BioLegend). Staining with biotin-conjugated antibodies was detected with Streptavidin-BV421 (1:100, BioLegend) or Streptavidin-PE-Cy7 (1:200, BD Pharmingen).

In experiments that examined aldehyde dehydrogenase activity, the Aldefluor assay was used (STEMCELL Technologies). Cells were incubated in the Aldefluor assay buffer containing BSA $(0.1 \%)$ and BAAA substrate $(0.12 \mu \mathrm{g} / \mathrm{mL})$ for 60 minutes at $37^{\circ} \mathrm{C}$. Thereafter, cells were washed in ice cold IMDM supplemented with FCS (2\%), Aldefluor assay buffer (10\%) and stained with antibodies against various cell surface markers, as described above. An aldehyde dehydrogenase inhibitor, DEAB $(0.75 \mathrm{nM})$-treated sample was used as a negative control.

Flow cytometry and cell sorting data were analyzed using FlowJo software (Tree Star).

\subsection{Quantitative real-time PCR}

Total RNA was prepared with the RNAqueous RNA Isolation Kit (ThermoFisher) that included treatment with RNase-free DNase. Reverse transcribed to cDNA was performed using the iScript cDNA Synthesis Kit (Bio-Rad). RT-qPCR was performed on a CFX384 Touch Real-Time PCR Detection System (Bio-Rad) with the QuantiFast SYBR Green PCR Kit (Qiagen). Gene expression was evaluated as $\triangle \mathrm{Ct}$ relative to $T B P$. For globin analyses, gene expression was evaluated as $\triangle \mathrm{Ct}$ relative to $A C T B$. Genomic DNA content was assessed using primers for the GAGEBI promoter. Primer sequences are listed in Table 2.3. 
Table 2.3 Primer sequences for RT-qPCR.

\begin{tabular}{|l|l|l|}
\hline Gene & Forward primer sequence (5'-3') & Reverse primer sequence (5'-3') \\
\hline TBP & TGAGTTGCTCATACCGTGCTGCTA & CCCTCAAACCAACTTGTCAACAGC \\
\hline GCTB & AAACTGGAACGGTGAAGGTGACAG & CAATGTGCAATCAAAGTCCTCGGC \\
\hline RUNX1a/b & CAACATACCTCATAGCATTATACAAGAC & CCCTATGTTCCTGGTTCTTCATATT \\
\hline SCL & CCGTGGATTCGCTTGAGTTA & GAAAGAAGAGGGAGCCAGAAG \\
\hline NOTCH1 & CGGGTCCACCAGTTTGAATG & GTTGTATTGGTTCGGCACCAT \\
\hline NOTCH2 & TTTGGCAACTAACGTAGAAACTCAAC & TGCCAAGAGCATGAATACAGAGA \\
\hline NOTCH3 & CTCCTGCATCCTTGCCTTG & GACTGAGAGGGTGGGTGGTA \\
\hline NOTCH4 & CATTAAAAGGCAGGCTGGAA & CGTGGAAGATGTCTGCTCTG \\
\hline$H E S 1$ & AGCTGGTGCTGATAACAGCGGAAT & CTACTGAGCAAGTGCTGAGGGTTT \\
\hline$H E S 5$ & TGGGTGCCTCCACTATGATCCTTA & GCTTCCACGTGACTGAGAGTTCAA \\
\hline$H E Y 1$ & TGATCATGGTGTGCGAGTGGTCAA & GCAACCACAGTTCCATGCACCAAA \\
\hline$H E Y 2$ & GAGTGAGAGAGTCGTGTTTC & ACTTCTGTCCCTTTCCTTTC \\
\hline$H B E$ & TCTGGCTACTCACTTTGGCAAGGA & TCACAGGAACACCTGCAAACTGGA \\
\hline$H B G 1 / 2$ & TGGGAAATGTGCTGGTGACCGTTT & AAGCTCTGAATCATGGGCAGTGAG \\
\hline$H B B$ & ACTAAGCTCGCTTTCTTGCTGTCC & TCCAGATGCTCAAGGCCCTTCATA \\
\hline
\end{tabular}




\subsection{Hemangioblast colony-forming assay}

Hemangioblast colony-forming potential was performed by plating 10,000 to 20,000 cells in $1 \%$ methylcellulose (1\%, Shin-Etsu) supplemented with FCS (10\%), D4T endothelial cell conditioned-medium (20\%), L-glutamine (2 $\mathrm{mM})$, ascorbic acid $(25 \mu \mathrm{g} / \mathrm{mL})$, transferrin $(150$ $\mu \mathrm{g} / \mathrm{mL})$, monothioglycerol (33 $\mu \mathrm{g} / \mathrm{mL})$, FGF2 (10 ng/mL), VEGF (10 $\mathrm{ng} / \mathrm{mL})$, IL6 (10 ng/mL), IL11 $(5 \mathrm{ng} / \mathrm{mL}), \mathrm{SCF}(100 \mathrm{ng} / \mathrm{mL})$ and SB431542 $(6 \mu \mathrm{M})$. Cultures were maintained in hypoxic conditions $\left(37^{\circ} \mathrm{C}, 5 \% \mathrm{CO}_{2}, 5 \% \mathrm{O}_{2}\right)$ for 6 days prior to quantification.

\subsection{Hematopoietic colony-forming assay}

Colony-forming progenitor number was quantified by plating 100 to 80,000 cells in methylcellulose (1\%) containing plasma-derived serum (15\%, Animal Technologies), protein-free hybridoma media II (5\%, Invitrogen), L-glutamine ( $2 \mathrm{mM})$, ascorbic acid ( $25 \mu \mathrm{g} / \mathrm{mL})$, transferrin (150 $\mu \mathrm{g} / \mathrm{mL})$, monothioglycerol (33 $\mu \mathrm{g} / \mathrm{mL})$, TPO (50 ng/mL), IL3 (50 ng/mL), IL6 (10 ng/mL), IL11 (5 ng/mL), SCF (100 ng/mL), EPO (4 Units/mL), GM-CSF (1 ng/mL), M-CSF (10 ng/mL), IGF1 $(25 \mathrm{ng} / \mathrm{mL})$, VEGF $(10 \mathrm{ng} / \mathrm{mL})$ and FGF2 $(10 \mathrm{ng} / \mathrm{mL})$. Cultures were maintained in normoxic conditions $\left(37^{\circ} \mathrm{C}, 5 \% \mathrm{CO}_{2}\right)$ for 9 to 14 days prior to quantification.

\subsection{OP9-DL4 co-culture for T lymphoid differentiation}

OP9 stromal cells that constitutively express Delta-like 4 (OP9-DL4) (Schmitt and ZunigaPflucker, 2002, 2006) were used to assay T lymphoid potential. Co-cultures were maintained in normoxic conditions $\left(37^{\circ} \mathrm{C}, 5 \% \mathrm{CO}_{2}\right) .1$ to 250,000 cells were cultured with OP9-DL4 cells on $0.1 \%$ gelatin-treated tissue culture plates in aMEM (Gibco) supplemented with penicillin/streptomycin (1\%), FCS (20\%, HyClone), L-glutamine (2 mM), IL7 (5 ng/mL) and FLT3L $(5 \mathrm{ng} / \mathrm{mL})$. SCF $(30 \mathrm{ng} / \mathrm{mL})$ was included at the start of the co-culture and removed after 4-6 days. Cultures were transferred to new OP9-DL4 cells every 4-6 days by vigorous pipetting and passage through a $40 \mu \mathrm{m}$ strainer. Cultures were analyzed by flow cytometry at the indicated stages and scored positive if greater than $10 \mathrm{CD} 45^{+} \mathrm{CD} 56^{-} \mathrm{CD} 4^{+} \mathrm{CD} 8^{+}$events were observed.

\subsection{HUVEC-E4ORF1 co-culture for hematopoietic cell expansion}

Human umbilical vein endothelial cells (HUVECs) that were engineered to express E4ORF1 (HUVEC-E4ORF1) (Seandel et al., 2008) were used to assay hematopoietic cell expansion. Co- 
cultures were maintained in normoxic conditions $\left(37^{\circ} \mathrm{C}, 5 \% \mathrm{CO}_{2}\right) .25$ cells were cultured with HUVEC-E4ORF cells on Collagen type $1(0.3 \mathrm{mg} / \mathrm{mL}$, Fujifilm)-treated tissue culture plates in Stemspan SFEM (STEMCELL Technologies) supplemented with L-glutamine (2 mM), TPO (50 ng/mL), SCF (100 ng/mL), VEGF (10 ng/mL), FGF2 (10 ng/mL), FLT3L (20 ng/mL), IL3 (50 $\mathrm{ng} / \mathrm{mL})$, IL7 $(20 \mathrm{ng} / \mathrm{mL})$, IL6 (10 ng/mL). Cultures were analyzed by flow cytometry at the indicated stages.

\subsection{Hematopoietic multipotent progenitor cell assay}

Hematopoietic cells were isolated from OP9-DL4 co-cultures initiated with day 6 CD34 ${ }^{+}$CD43cells after 5 days. Single cells were sorted into the wells of 96-well plates retaining index sorting information on an AriaII-RITT cell sorter. The perimeter wells were excluded from the sort. The cells were cultured in $\alpha$-MEM supplemented with FCS (20\%), penicillin/streptomycin (1\%, ThermoFisher), L-glutamine (2 mM, ThermoFisher) and SCF (30 ng/mL), IL7 (5 ng/mL) and FLT3L $(5 \mathrm{ng} / \mathrm{mL})$. The cultures were maintained in normoxic conditions $\left(37^{\circ} \mathrm{C}, 5 \% \mathrm{CO}_{2}\right)$. After 4 days, the cultures were dissociated with Trypsin and 30\% of the culture was transferred to methylcellulose to evaluate erythro-myeloid potential as described in Section 2.8. The cultures were scored after 14 days and erythroid colonies were picked for $\beta$ globin expression analysis by RT-qPCR as described in Section 2.6. As the OP9-DL4 cells express GFP, the colonies were visualized under fluorescence to confirm their human identity. The remaining fraction of the culture was seeded on new OP9-DL4 cells to evaluate NK and T cell potential as described in Section 2.9. Cultures were dissociated with trypsin and transferred to fresh OP9-DL4 biweekly for another 12 to 14 days. OP9-DL4 co-cultures were analyzed by flow cytometry. Greater than 10 $\mathrm{CD} 45^{+}$-gated events $\left(\mathrm{T}: \mathrm{CD}^{+} \mathrm{CD}^{-} 6^{-} \mathrm{CD} 4^{+} \mathrm{CD} 8^{+}, \mathrm{NK}: \mathrm{CD} 6^{+} \mathrm{CD}^{+}\right)$were required to call a positive well.

\subsection{Hematopoietic cell xenotransplantation}

Animal experiments were performed in accordance to the institution guidelines approved by the University Health Network Animal Care Committee. Post-natal day 3 NOD-

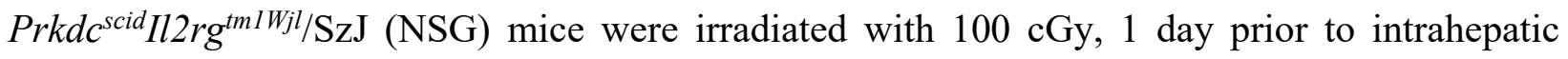
transplantation with cryopreserved CD34 ${ }^{+}$cord blood (25,000 cells, STEMCELL Technologies) and hPSC-derived $\mathrm{CD} 34^{+} \mathrm{CD} 45^{+} \mathrm{CD} 90^{+} \mathrm{CD}^{-}$cells $(4,000-9,000$ cells). Four weeks after transplantation, mice were euthanized and the femurs were flushed through a $40 \mu \mathrm{m}$ filter in 
IMDM to collect the marrows. Prior to antibody staining, the cells were incubated with anti-mouse CD16/32 (1:20, clone 93, ThermoFisher) for 10 minutes at $4^{\circ} \mathrm{C}$. To reliably identify human cells, two human-specific CD45 antibody clones were used.

\subsection{Lentiviral transduction of hPSC-derived cells}

EBs from day 4 of differentiation were dissociated and sorted as described in Section 2.5. Thereafter, the sorted populations were resuspended at $1,000,000$ to $3,000,000$ cells $/ \mathrm{mL}$ in StemPro-34 media prior to lentiviral transduction. The transduction volume was limited to $1.5 \mathrm{~mL}$ and performed in $5 \mathrm{~mL}$ polypropylene tubes (Falcon). Concentrated lentivirus $\left(6.22 \times 10^{8}\right.$ to $8.63 \times 10^{8}$ ) that constitutively expresses eGFP from the EF1 $\alpha$ promoter (Tailored Genes) was used. The multiplicity of infection (MOI) was limited to 2.0 to ensure that only a small fraction of the cells was transduced. Infection was accomplished by spinoculation at $900 \mathrm{rcf}$ for 30 minutes at room temperature. Thereafter, the cells were washed with IMDM supplemented with penicillin/streptomycin (1\%) and FCS (2\%) and resuspended at 250,000 cells/mL in StemPro-34 media supplemented with recombinant factors appropriate to day 4 of differentiation. Cells were aggregated and cultured for 3 days prior to FACS to isolate $\mathrm{GFP}^{+}$cells as described in Sections 2.4 and 2.5. In addition, 10,000 to 25,000 cells were plated in methylcellulose to assay hemangioblast colony-forming potential as described in Section 2.7.

\subsection{Quantification and statistical analyses}

All data are presented as the mean \pm the standard error of the mean (s.e.m.). Statistical analyses were performed in GraphPad Prism 8 or the R statistical computing environment as indicated in the figure captions. 


\section{Chapter 3}

\section{Architecture of human yolk sac hematopoietic development using hPSCs}

\subsection{Acknowledgements}

I thank Deborah French (Children's Hospital of Philadelphia) for the CHOP10WT iPSC line, Feng Zhang for the PX458 pSpCas9(BB)-2A-GFP plasmid (Addgene plasmid 48138), Nestor Fernandez for the CRISPR-Cas9 targeting strategy to knockout MYB from hPSCs, the SickKids/UHN Flow Cytometry Facility for their assistance with cell sorting, The Centre for Applied Genomics (TCAG) for their assistance with Sanger sequencing, Cell Line Genetics for the karyotypic analysis and Shahin Rafii (Weill Cornell Medicine) for the HUVEC-E4ORF1 endothelial cell line.

\subsection{Primer}

Analyses of model organism development have demonstrated that embryonic hematopoiesis consists of distinct programs that differ in spatiotemporal organization and lineage potential. Although long thought to primarily fulfil the hematopoietic requirements, such as the oxygen demand, of the rapidly developing early embryo, studies over the past decade have provided strong evidence that the progeny of the yolk sac hematopoietic programs contribute to tissue-resident immune cell populations in the fetus and adult. These immune cell populations serve essential tissue-specific homeostatic functions and their dysregulation can lead to disease. Access to the progenitors of these populations would enable studies of their function in normal development and disease and provide the basis for the design of novel cell-based therapies. For these applications to be tested and realized, it is essential to generate these cell types from their appropriate source. As these populations are specified at such early stages of development, hPSCs represent the sole source of their transient hematopoietic progenitor populations to study their biology and their role in disease processes. Additionally, access to these lineages will provide the cells necessary to develop novel cell-based therapies to treat diseases that impact the function of tissue-resident immune cells in the adult. In this study, I describe a model of the development and regulation of the human primitive, EMP and LMP programs using hPSC differentiation. I provide an efficient protocol to generate the hematopoietic progenitors of these embryonic hematopoietic programs 
from $\mathrm{KDR}^{+} \mathrm{CD} 235 \mathrm{a} / \mathrm{b}^{+}$mesoderm and apply it to uncover novel regulatory roles for $\mathrm{NOTCH}$ and MYB in the generation of the human primitive hematopoietic program. In addition, I demonstrate that $\mathrm{KDR}^{+} \mathrm{CD} 235 \mathrm{a} / \mathrm{b}^{+}$mesoderm also gives rise to progenitors with $\mathrm{T}$ lymphoid potential and show that this lineage develops from a multipotent hematopoietic progenitor with NK cell, T lymphoid, EMP erythroid and myeloid potential. Together, these findings link the human EMP and LMP programs and provide evidence for a common yolk sac-derived multipotent progenitor (MPP) program in the human. With the advances in our understanding of human developmental hematopoiesis from this thesis, we can now better interrogate the development and function of tissue-resident immune cells and have facilitated access to populations with therapeutic potential.

\subsection{Results}

\subsubsection{Specification of the primitive hematopoietic program}

Our previous studies have shown that BMP4, together with FGF2 and either with low levels of Activin A or the Wnt inhibitor, IWP2 promotes the generation of $\mathrm{KDR}^{+} \mathrm{CD} 235 \mathrm{a}^{+}$(herein referred to as $\mathrm{CD} 235 \mathrm{a} / \mathrm{b}$ to be consistent with the specificity of the antibody clone that was used) mesoderm that gives rise to primitive hematopoietic cells and some lineages of an EMP-like program. However, this mesoderm did not show any capacity to generate T lymphoid cells (Sturgeon et al., 2014) suggesting that it did not possess the full spectrum of hematopoietic potential observed in the mouse yolk sac. These findings may indicate that hematopoietic development of the mouse and human are different or that the CD235a/ $\mathrm{b}^{+}$mesoderm in the previous study was not properly specified. To address this, I followed the lead of studies of cardiovascular development from our lab that demonstrated that specific concentrations of BMP4 and Activin A efficiently induce $\mathrm{CD} 235 \mathrm{a} / \mathrm{b}^{+}$mesoderm with ventricular cardiomyocyte potential (Lee et al., 2017). To understand whether a similar strategy can be used to generate $\mathrm{KDR}^{+} \mathrm{CD} 235 \mathrm{a} / \mathrm{b}^{+}$mesoderm that gives rise to the primitive hematopoietic program, I titrated Activin A (0 to $10 \mathrm{ng} / \mathrm{mL}$ ) in the presence of BMP4 $(10 \mathrm{ng} / \mathrm{mL})$ and FGF2 (5 ng/mL) from day 1 of differentiation (Figure 3.1a). Consistent with the observations of Lee et al. (2017), I observed that the frequency of CD235a/ $\mathrm{b}^{+}$cells increased in an Activin A dose-dependent manner and plateaued when its concentration exceeded $6 \mathrm{ng} / \mathrm{mL}$. Although the majority of the cells did not express KDR at day 3 of differentiation (Figure $3.1 \mathrm{~b}$ and 3.1c), they did upregulate its expression by day 4 of differentiation. Under these conditions, cells 
A

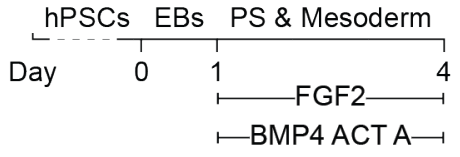

B

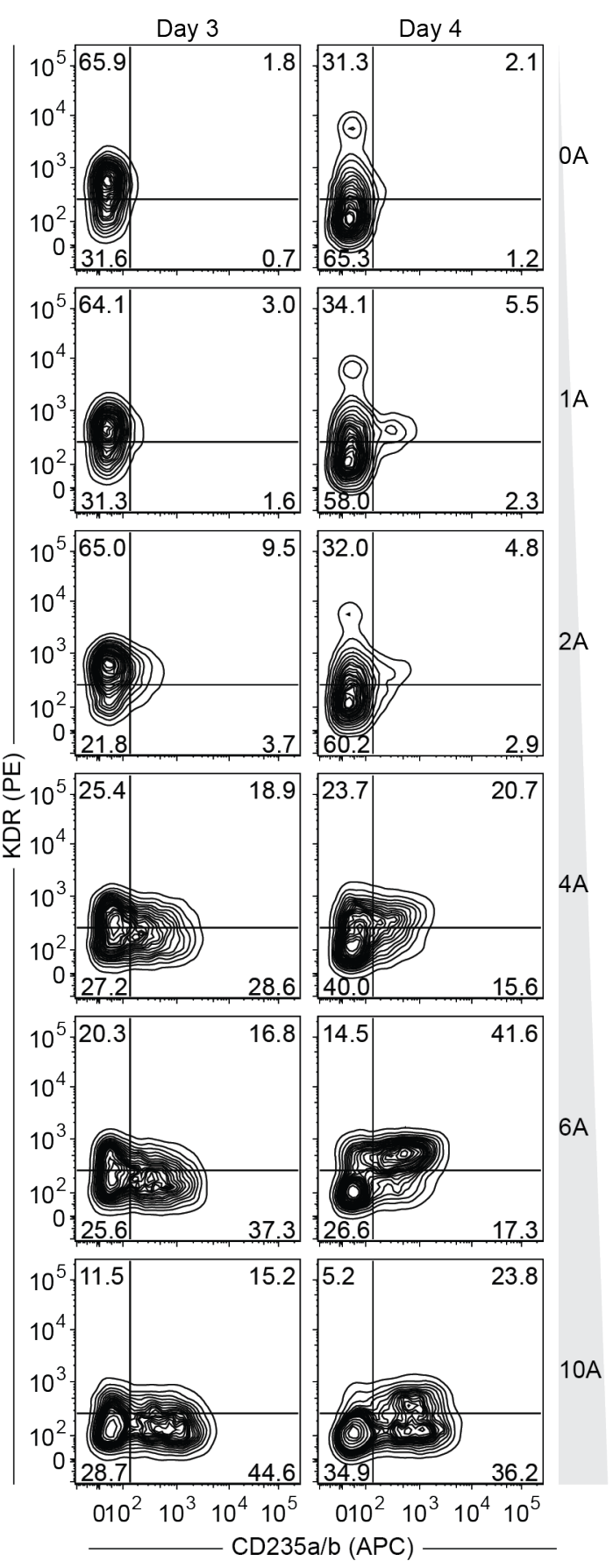

C

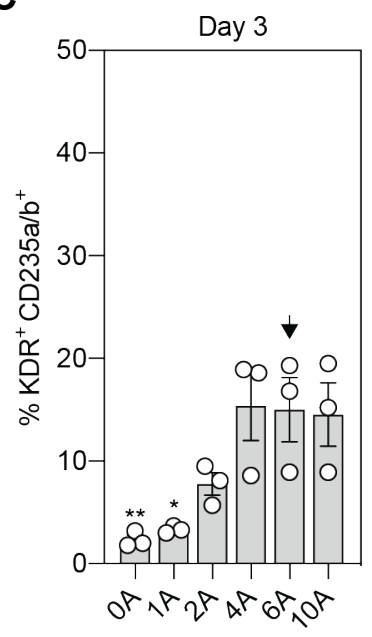

D

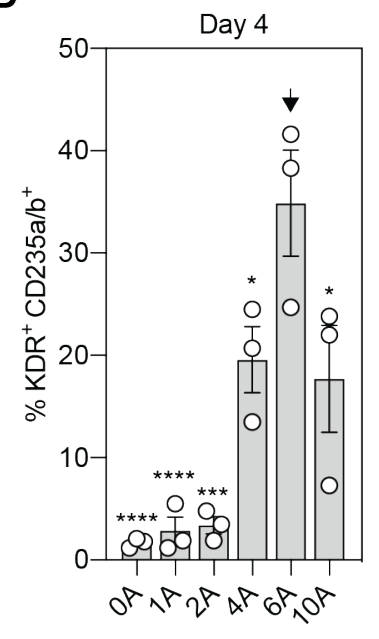


Figure 3.1 Induction of $\mathrm{KDR}^{+} \mathrm{CD} 235 \mathrm{a} / \mathrm{b}^{+}$mesoderm.

A. Schematic of mesoderm specification from hPSCs through the addition of BMP4, FGF2 and Activin A (A) on day 1 of differentiation.

B. Representative flow cytometric analysis of KDR and CD235a/b expression on days 3 and 4 of differentiation.

C. Quantification of the proportion of $\mathrm{KDR}^{+} \mathrm{CD} 235 \mathrm{a} / \mathrm{b}^{+}$cells on day 3 of differentiation $(\mathrm{n}=3)$. ANOVA. $* P<0.05$ and $* * P<0.01$ versus cultures induced with $6 \mathrm{ng} / \mathrm{mL}$ of Act A.

D. Quantification of the proportion of $\mathrm{KDR}^{+} \mathrm{CD} 235 \mathrm{a} / \mathrm{b}^{+}$cells on day 4 of differentiation $(\mathrm{n}=3)$. ANOVA. ${ }^{*} P<0.05, * * * P<0.001$ and $* * * * P<0.0001$ versus cultures induced with $6 \mathrm{ng} / \mathrm{mL}$ of Act A. 
induced with $10 \mathrm{ng} / \mathrm{mL}$ of BMP4, $5 \mathrm{ng} / \mathrm{mL}$ of FGF2 and $6 \mathrm{ng} / \mathrm{mL}$ of Activin A consistently gave rise to cultures that contained greater than $30 \%$ of $\mathrm{KDR}^{+} \mathrm{CD} 235 \mathrm{a} / \mathrm{b}^{+}$cells (Figure $3.1 \mathrm{~b}$ and $3.1 \mathrm{~d}$ ).

To determine whether the cultures induced with BMP4, FGF2 and $6 \mathrm{ng} / \mathrm{mL}$ of Activin A had hematopoietic potential, I cultured the EBs in the presence of VEGF, FGF2 and hematopoietic cytokines (Figure 3.2a) and analyzed the cultures by flow cytometry over 9 days at 3-day intervals for the emergence of hematopoietic cells marked by the expression of CD43 and CD45. Hematopoietic cells were present at day 6 of differentiation and increased in number over the next 9 days of culture (Figure 3.2b and 3.2c) indicative of the expansion and maturation of the primitive hematopoietic program. To formally determine whether the hematopoietic cells belonged to the primitive hematopoietic program, I assayed colony-forming progenitor potential in methylcellulose cultures. The number of colony-forming progenitors rapidly increased between days 6 and 9 of differentiation and then decreased over the next 6 days (Figure 3.2d). Erythroid progenitors that generated small, compact colonies of erythroblasts dominated the cultures (Figure $3.2 \mathrm{~d}$ and 3.2e). RT-qPCR revealed that the cells within these colonies predominantly expressed the embryonic $\beta$ globin, $H B E$ (Figure $3.2 \mathrm{f}$ ) confirming that they represent the human primitive erythroid lineage.

To formally demonstrate that the primitive hematopoietic cells develop from $\mathrm{KDR}^{+} \mathrm{CD} 235 \mathrm{a} / \mathrm{b}^{+}$ mesoderm, I isolated the CD235a/ $\mathrm{b}^{+}$and $\mathrm{CD} 235 \mathrm{a} / \mathrm{b}^{-}$fractions of $\mathrm{KDR}^{+}$population by $\mathrm{FACS}$ at day 4 of differentiation and cultured them in the presence of VEGF, FGF2 and hematopoietic cytokines for 5 days (Figure 3.3a). The two $\mathrm{KDR}^{+}$populations generated $\mathrm{CD} 43^{+}$hematopoietic cells that increased in number over 5 days of culture (Figure $3.3 \mathrm{~b}$ and $3.3 \mathrm{c}$ ). The populations generated comparable numbers of colony-forming progenitors, the majority of which gave rise to small colonies of primitive erythroblasts (Figure 3.3d). Analyses of hemangioblast potential revealed that the frequency of BL-CFCs was equal between the two $\mathrm{KDR}^{+}$populations (Figure 3.3e). However, given the higher proportion of $\mathrm{KDR}^{+} \mathrm{CD} 235 \mathrm{a} / \mathrm{b}^{+}$cells relative to $\mathrm{KDR}^{+} \mathrm{CD} 235 \mathrm{a} / \mathrm{b}^{-}$ cells, the majority of BL-CFCs are contained within the $\mathrm{KDR}^{+} \mathrm{CD} 235 \mathrm{a} / \mathrm{b}^{+}$population. The presence of primitive erythroid progenitors and BL-CFCs in the two $\mathrm{KDR}^{+}$populations demonstrate that both have primitive hematopoietic potential. This suggests that either primitive hematopoiesis is not restricted to the $\mathrm{KDR}^{+} \mathrm{CD} 235 \mathrm{a} / \mathrm{b}^{+}$mesoderm or that the day 4 $\mathrm{KDR}^{+} \mathrm{CD} 235 \mathrm{a} / \mathrm{b}^{-}$population developed from day 3 mesoderm that expressed $\mathrm{CD} 235 \mathrm{a} / \mathrm{b}$. 
Collectively, these data demonstrate that $\mathrm{KDR}^{+} \mathrm{CD} 235 \mathrm{a} / \mathrm{b}^{+}$mesoderm gives rise to the progenitors of the primitive hematopoietic program. 
A

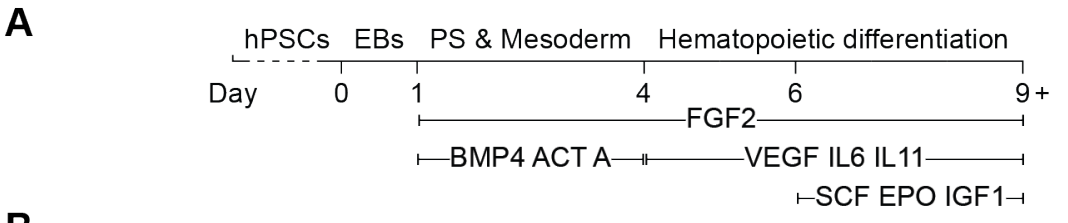

B
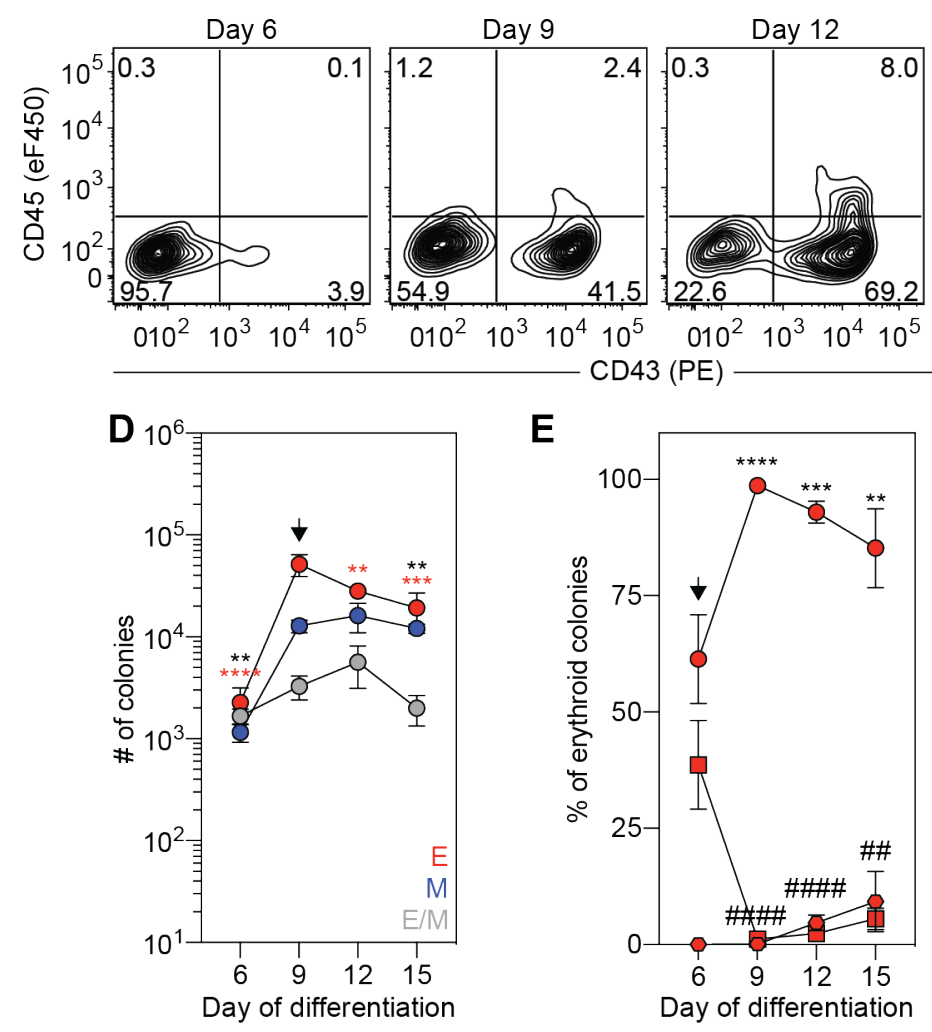

E

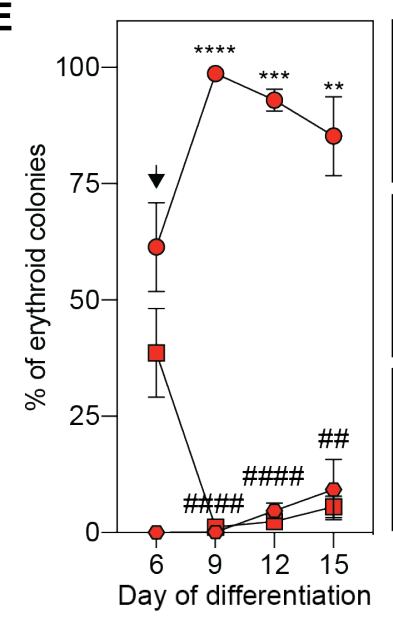

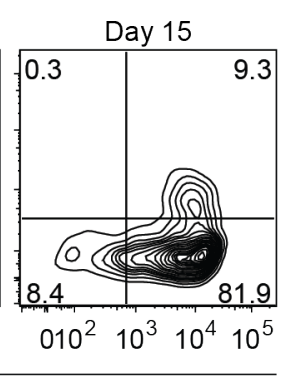
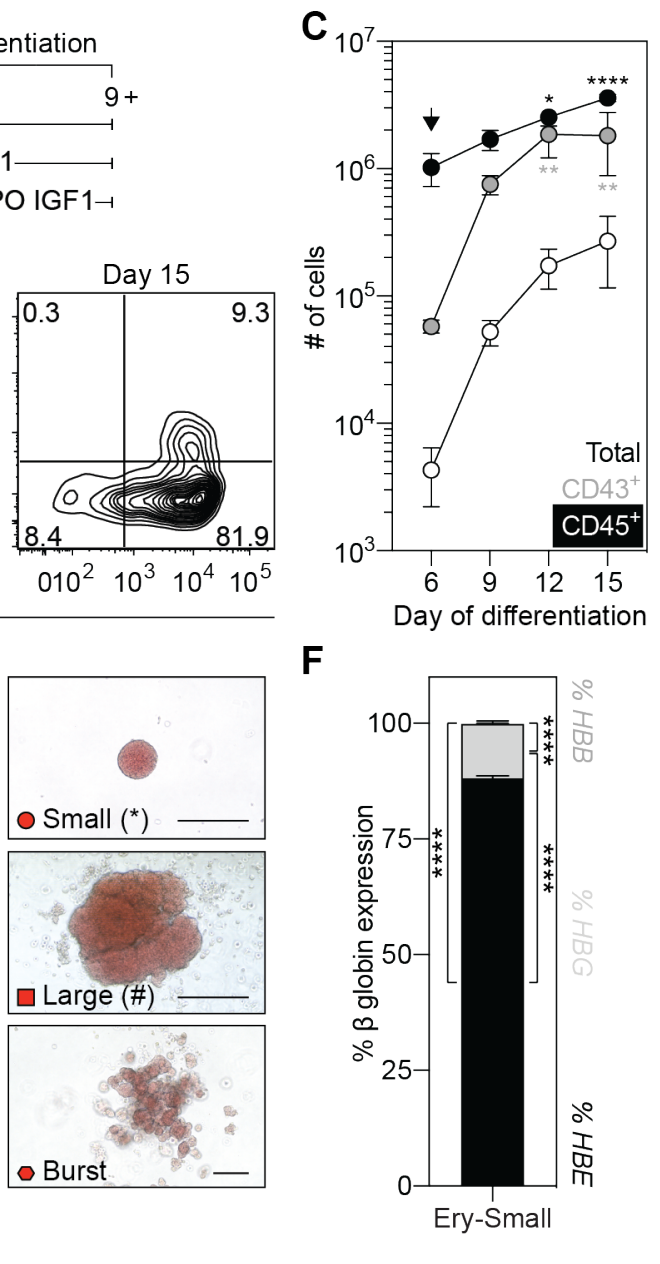

$\mathbf{F}$

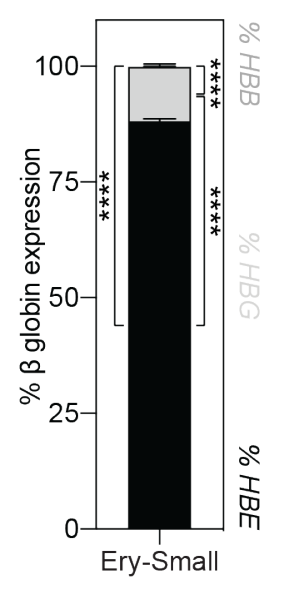

Figure 3.2 Emergence of the primitive hematopoietic program from hPSCs.

A. Schematic of hematopoietic differentiation from hPSCs through the addition of BMP4, FGF2 and ACT A followed by culture in the presence of VEGF, FGF2 and hematopoietic cytokines.

B. Representative flow cytometric analysis of CD43 and CD45 expression between days 6 and 15 of differentiation.

C. Quantification of the number of total, $\mathrm{CD} 43^{+}$and $\mathrm{CD} 45^{+}$cells generated from 500,000 input $\mathrm{H} 1 \mathrm{hESCs}$ in the cultures at the indicated days $(\mathrm{n}=4)$. ANOVA. $* P<0.05, * * P<0.01$ and $* * * * P<0.0001$ versus the indicated population on day 6 of differentiation.

D. Colony-forming progenitor numbers generated between days 6 and 15 of differentiation based on an input of 500,000 H1 hESCs $(\mathrm{n}=3)$. ANOVA. $* * P<0.01, * * * P<0.001$ and $* * * * P<$ 0.0001 versus the indicated lineage on day 9 of differentiation (black $=$ all colonies). Colonies: $\mathrm{E}=$ erythroid (red), $\mathrm{M}=$ myeloid (blue) and $\mathrm{E} / \mathrm{M}=$ mixed erythro-myeloid (grey).

E. Distribution of erythroid progenitors generated between days 6 and 15 of differentiation $(\mathrm{n}=$ 3). ANOVA. $* * P<0.01, * * * P<0.001$ and $* * * * P<0.0001$, and $\# \# P<0.01$ and $\# \# \# P<$ 0.0001 versus small and large erythroid colony morphologies, respectively.

F. RT-qPCR analysis of the percentage of $H B E, H B G$ and $H B B \beta$ globin expression of small erythroid colonies $(\mathrm{n}=3)$. ANOVA. $* * * * P<0.001$ versus the indicated $\beta$ globin gene. 

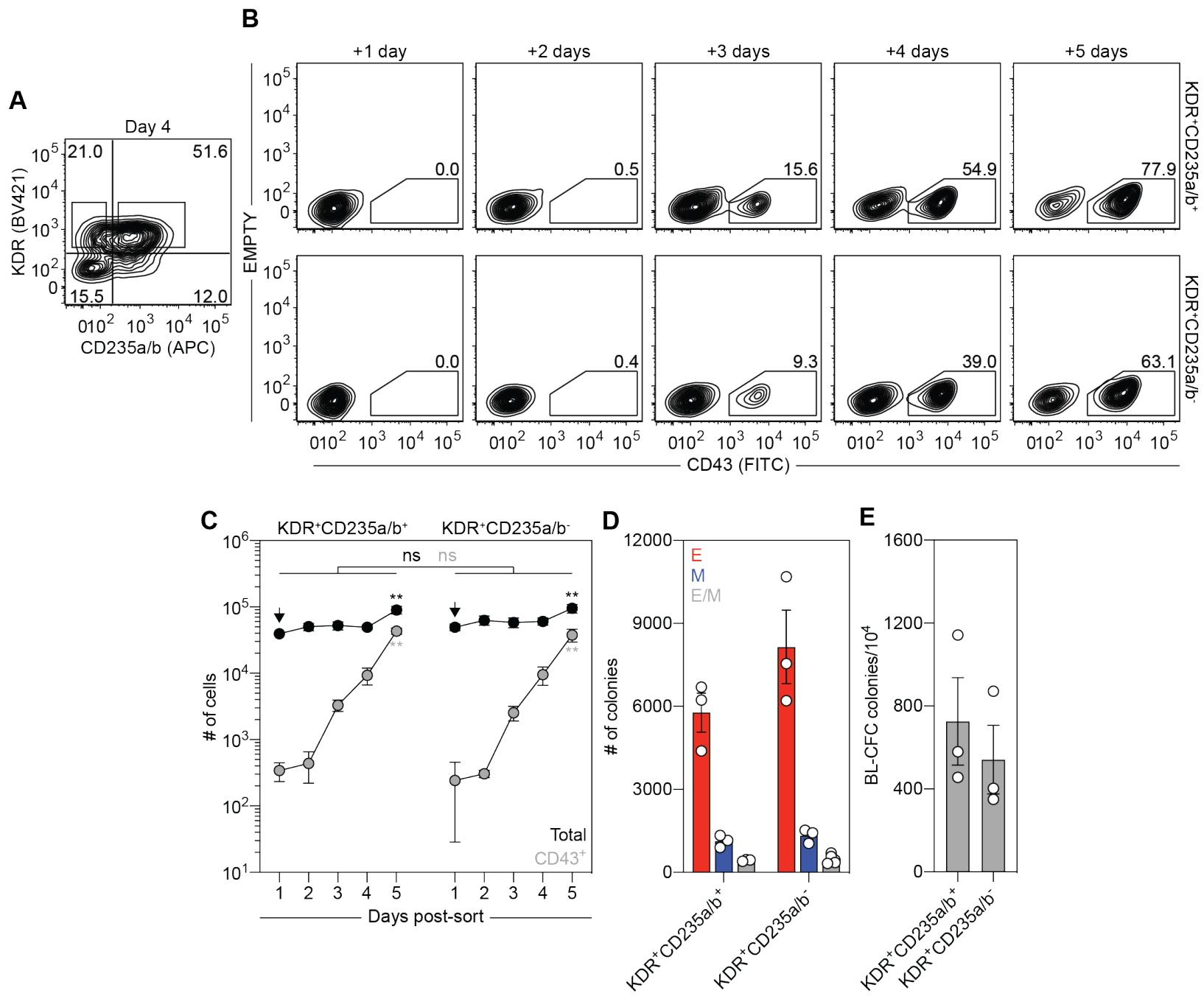

E

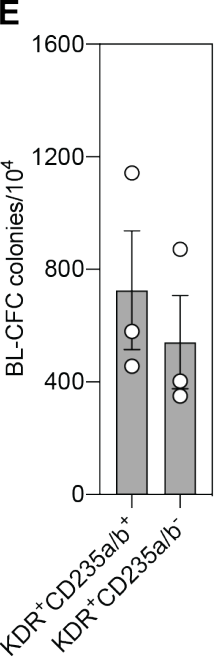

Figure 3.3 Hematopoietic potential of $\mathrm{KDR}^{+}$mesoderm.

A. Gating strategy used to isolate $\mathrm{KDR}^{+}$mesoderm based on $\mathrm{CD} 235 \mathrm{a} / \mathrm{b}$ expression on day 4 of differentiation.

B. Representative flow cytometric analysis of CD43 expression over 5 days in populations generated from the $\mathrm{KDR}^{+}$fractions.

C. Quantification of the number of total and $\mathrm{CD}_{4} 3^{+}$cells generated from 62,500 day $4 \mathrm{KDR}^{+}$cells over 5 days of culture $(\mathrm{n}=3)$. ANOVA. ${ }^{* *} P<0.01$ versus the indicated sample.

D. Colony-forming progenitor number after 5 days of culture of 62,500 day $4 \mathrm{KDR}^{+}$cells $(\mathrm{n}=3)$. ANOVA. not significant. Colonies: $\mathrm{E}=$ erythroid (red), $\mathrm{M}=$ myeloid (blue) and $\mathrm{E} / \mathrm{M}=$ mixed erythro-myeloid (grey).

E. BL-CFC frequency of the $\mathrm{KDR}^{+}$populations on day 4 of differentiation $(\mathrm{n}=3)$. $\mathrm{t}$-test. not significant. 


\subsubsection{The primitive hematopoietic program transitions through a hemogenic endothelial cell intermediate}

Studies in the mouse have suggested that the primitive hematopoietic program transitions through a HEC intermediate (Lancrin et al., 2009; Stefanska et al., 2017). To determine whether the same holds true for the human primitive hematopoietic program, I monitored the expression of CD43 by flow cytometry to define earliest stage that primitive hematopoietic cells appear. As shown in Figure 3.4a, $\mathrm{CD}_{4} 3^{+}$cells were first observed on day 6 of differentiation. Analyses of the cultures 24 hours earlier, prior to the emergence of $\mathrm{CD} 43^{+}$cells, revealed the presence of a population that displayed the surface marker phenotype of HECs, including the expression of vascular markers, KDR, CD34, KIT, CD144 and CD31 and a lack of the hematopoietic markers, CD43 or CD45 (Figure $3.4 \mathrm{~b}$ and 3.4c). RT-qPCR analyses also showed that two transcription factors associated with HECs, RUNX1 and SCL/TAL1 were expressed in the $\mathrm{KDR}^{+} \mathrm{CD} 34^{+}$population (Figure 3.4d) further supporting the interpretation that this population contains HECs that give rise to the hematopoietic cells of the primitive hematopoietic program.

To test the HEC potential of the $\mathrm{KDR}^{+} \mathrm{CD} 34^{+}$population, I isolated it on day 5 of differentiation by FACS and cultured the cells as aggregates in the presence of VEGF, FGF2 and hematopoietic cytokines for 7 days (Figure 3.5a). I observed a small population of $\mathrm{CD} 43^{+}$hematopoietic cells after 1 day that became increasingly abundant over the duration of the culture (Figure $3.5 \mathrm{~b}$ and 3.5c). Analyses of colony-forming progenitor potential in methylcellulose showed transient progenitor activity within the aggregates that predominantly gave rise to small erythroid colonies characteristic of the primitive hematopoietic program (Figure 3.5d). To provide additional evidence that the primitive hematopoietic program transitions through a HEC intermediate, I plated the day $5 \mathrm{KDR}^{+} \mathrm{CD} 34^{+}$population on Matrigel to visualize EHT. An adherent monolayer formed within 1 day of culture that rapidly gave rise to a population of round, non-adherent $\mathrm{CD}_{4} 3^{+}$ hematopoietic cells (Figure 3.5e and 3.5f). Taken together, these results provide strong evidence that the human primitive hematopoietic program transitions through a HEC intermediate prior to the onset of hematopoiesis. 
A

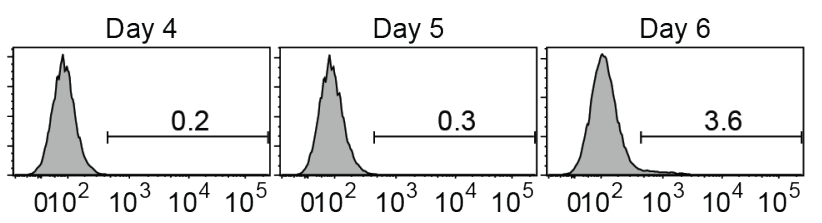

B CD43 (FITC)

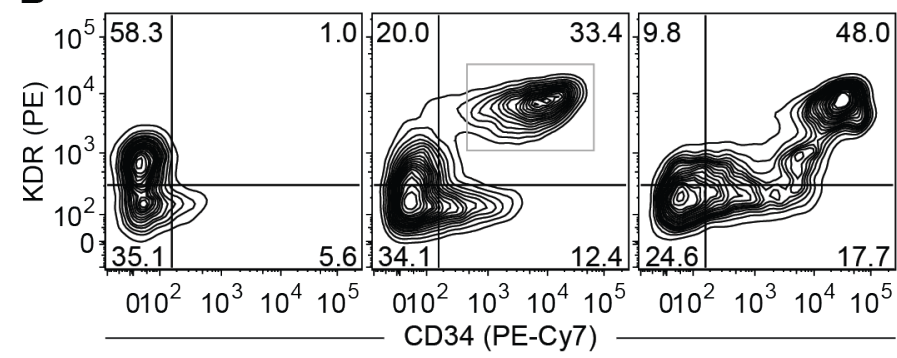

C
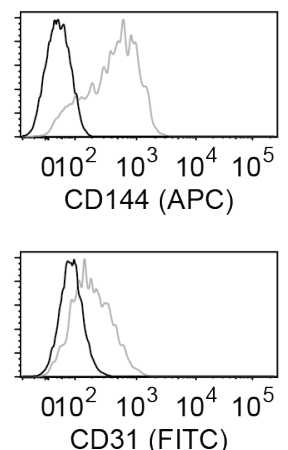
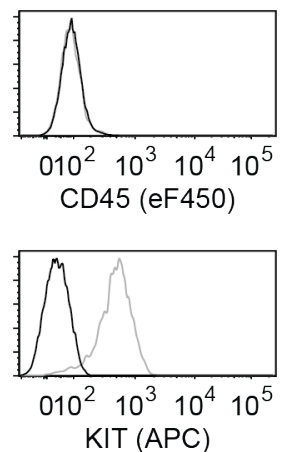

D
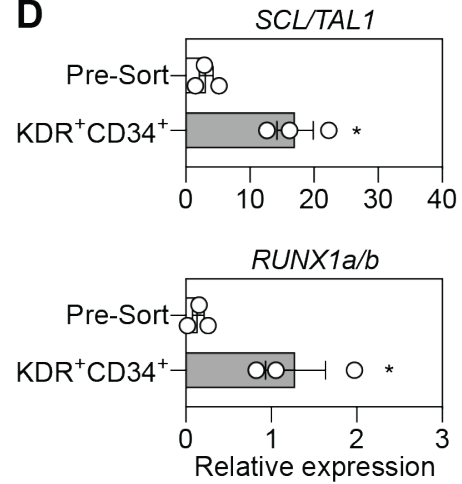

Figure 3.4 Characterization of $\mathrm{KDR}^{+} \mathrm{CD} 34^{+}$cells at day 5 of differentiation.

A. Representative flow cytometric analysis of CD43 expression between days 4 and 6 of differentiation.

B. Representative flow cytometric analysis of KDR and CD34 expression between days 4 and 6 of differentiation.

C. Representative flow cytometric analysis of KIT, CD144, CD31 and CD45 expression in the $\mathrm{KDR}^{+} \mathrm{CD} 34^{+}$population (gray) at day 5 of differentiation. Black line: unstained cells.

D. RT-qPCR analysis of $S C L / T A L 1$ and $R U N X 1 a / b$ expression in the $\mathrm{KDR}^{+} \mathrm{CD} 34^{+}$and unsorted populations on day 5 of differentiation $(\mathrm{n}=3)$. t-test. $* P<0.05$. 

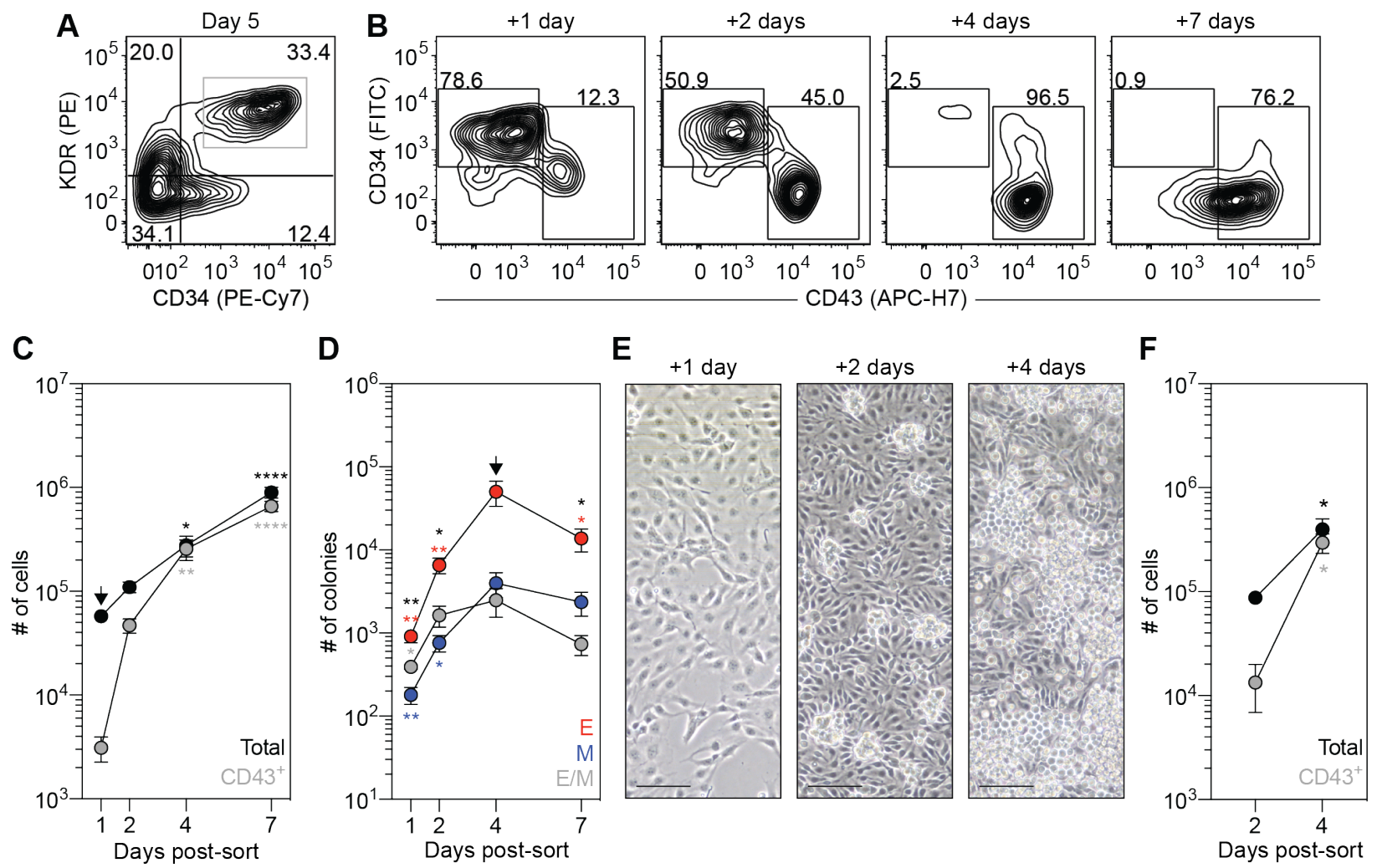

Figure 3.5 The primitive hematopoietic program transitions through a hemogenic endothelial cell intermediate.

A. Gating strategy (grey) used for the isolation the $\mathrm{KDR}^{+} \mathrm{CD} 34^{+}$population on day 5 of differentiation.

B. Representative flow cytometric analysis of CD34 and CD43 expression in populations generated from the day $5 \mathrm{KDR}^{+} \mathrm{CD} 34^{+}$isolated cells.

C. Quantification of the number of total and $\mathrm{CD}^{2} 3^{+}$cells generated from 62,500 day 5 $\mathrm{KDR}^{+} \mathrm{CD} 34^{+}$cells over 7 days of culture $(\mathrm{n}=4-5)$. ANOVA. ${ }^{*} P<0.05, * * P<0.01$ and $* * * * P$ $<0.0001$ versus the indicated population after 1 day of culture.

D. Colony-forming progenitor numbers generated from 62,500 day $5 \mathrm{KDR}^{+} \mathrm{CD} 34^{+}$cells over 7 of culture $(\mathrm{n}=4-5)$. ANOVA. $* P<0.05$ and $* * P<0.01$ versus the indicated lineage after 4 days of culture (black $=$ all colonies). Colonies: $\mathrm{E}=$ erythroid (red), $\mathrm{M}=$ myeloid (blue) and $\mathrm{E} / \mathrm{M}=$ mixed erythro-myeloid (grey).

E. Representative phase-contrast images of monolayer cultures derived from the day 5 $\mathrm{KDR}^{+} \mathrm{CD} 34^{+}$population over 4 days of culture. Scale bar $=50 \mu \mathrm{m}$.

F. Quantification of the number of total and $\mathrm{CD}_{4} 3^{+}$cells over 4 days in monolayer cultures generated from 20,000 day $5 \mathrm{KDR}^{+} \mathrm{CD} 34^{+}$cells $(\mathrm{n}=3)$. t-test. ${ }^{*} P<0.05$ versus the indicated population after 2 days of culture. 


\subsubsection{The primitive hematopoietic program is dependent on NOTCH signaling}

Studies of mouse embryonic hematopoiesis have demonstrated that Notch signaling is dispensable for the development of the primitive and EMP hematopoietic programs, but essential for the generation of HSCs (Hadland et al., 2004). To evaluate the role of NOTCH signaling in the development of the human primitive hematopoietic program, I analyzed the expression of the NOTCH receptors (NOTCH1, NOTCH2, NOTCH3, NOTCH4) and found that NOTCH1 and NOTCH4 were expressed in the $\mathrm{KDR}^{+} \mathrm{CD} 34^{+}$population at day 5 of differentiation (Figure 3.6a). To determine if this pathway is required for primitive hematopoiesis, I cultured isolated $\mathrm{KDR}^{+} \mathrm{CD} 34^{+}$cells as aggregates in the presence of VEGF, FGF2 and hematopoietic cytokines. Previous studies in our lab have shown that this format induces endogenous NOTCH signaling. RT-qPCR analyses confirmed this finding and showed elevated levels of expression of the NOTCH target genes, HES1, HEY1 and HEY2 during the first 2 days of culture suggesting that the emergence of the primitive hematopoietic program is regulated by NOTCH. The expression levels declined over the next 5 days of culture, coincident with the generation and expansion of primitive hematopoietic cells (Figure 3.6b). To formally test whether NOTCH signaling is required to generate the hematopoietic cells of the primitive hematopoietic program, I cultured the day 5

$\mathrm{KDR}^{+} \mathrm{CD} 34^{+}$population in the presence of gamma-secretase inhibitor (GSI) to inhibit NOTCH signaling. GSI treatment reduced the expression of NOTCH target genes (HES1, HEY1 and HEY2; Figure 3.7a) confirming the inhibition of the pathway. In contrast to our prediction based on studies of model organism development, the number of $\mathrm{CD} 43^{+}$hematopoietic cells and colony-forming progenitors were reduced in the presence of GSI (Figure $3.7 \mathrm{~b}$ to $3.7 \mathrm{~d}$ ) strongly suggesting that NOTCH signaling is required for the generation of the human primitive hematopoietic program. 


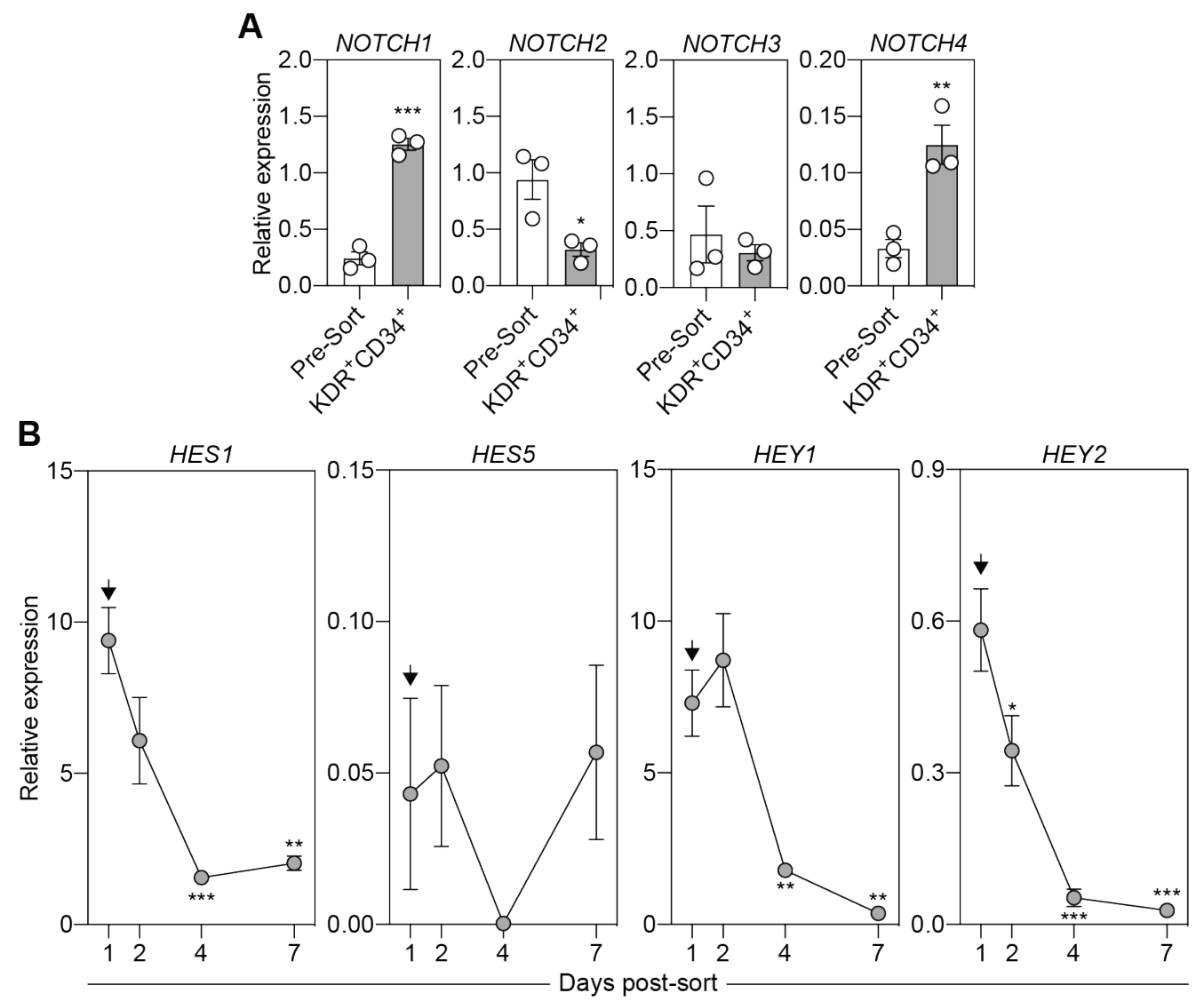

Figure $3.6 \mathrm{NOTCH}$ signaling during the development of the primitive hematopoietic program.

A. RT-qPCR analysis of NOTCH receptor expression in the day $5 \mathrm{KDR}^{+} \mathrm{CD} 34^{+}$and pre-sorted populations following 5 days of culture $(\mathrm{n}=3)$. t-test. $* P<0.05,{ }^{*} * P<0.01$ and $* * * P<0.001$.

B. RT-qPCR analysis of NOTCH target gene expression over 7 days of monolayer culture of the day $5 \mathrm{KDR}^{+} \mathrm{CD} 34^{+}$cells $(\mathrm{n}=3)$. ANOVA. ${ }^{*} P<0.05,{ }^{* *} P<0.01$ and ${ }^{* * *} P<0.001$ versus day 1 of culture. 

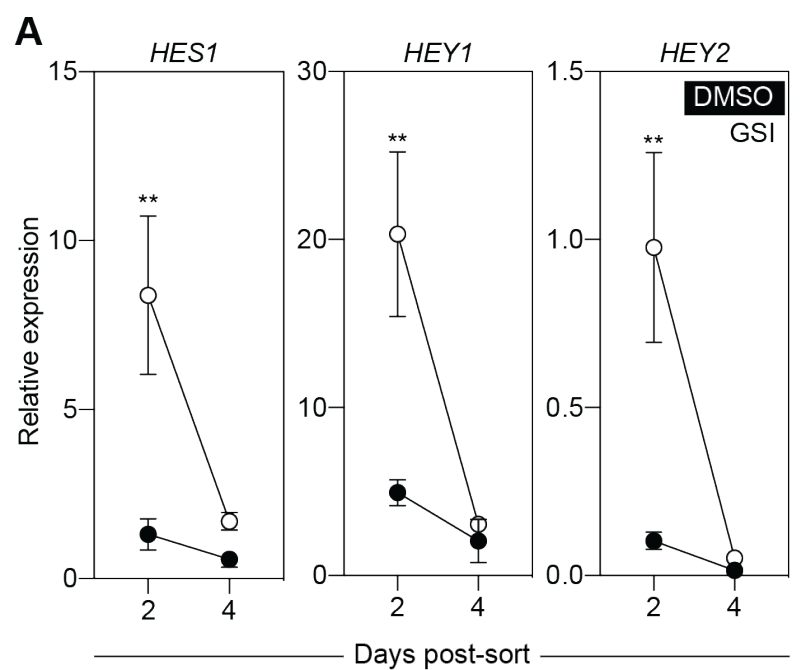

B
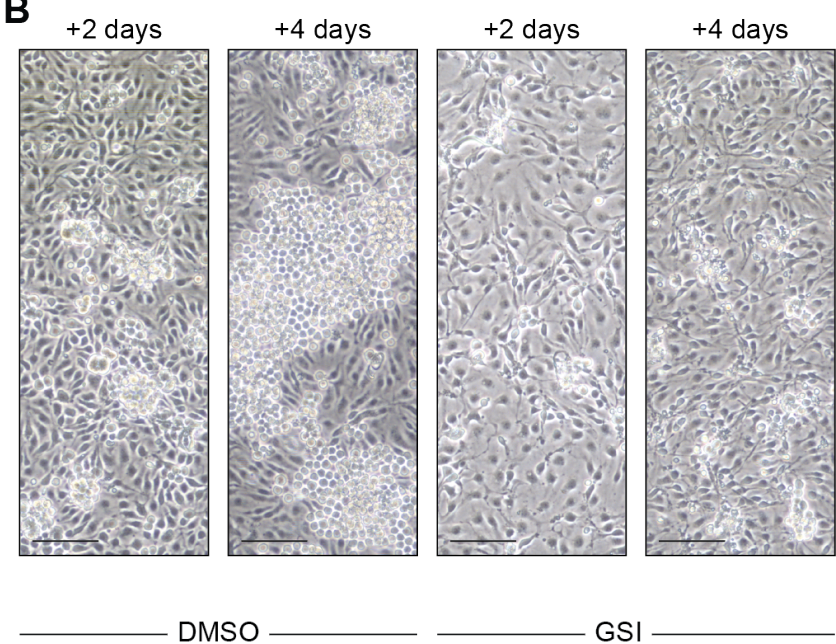

C
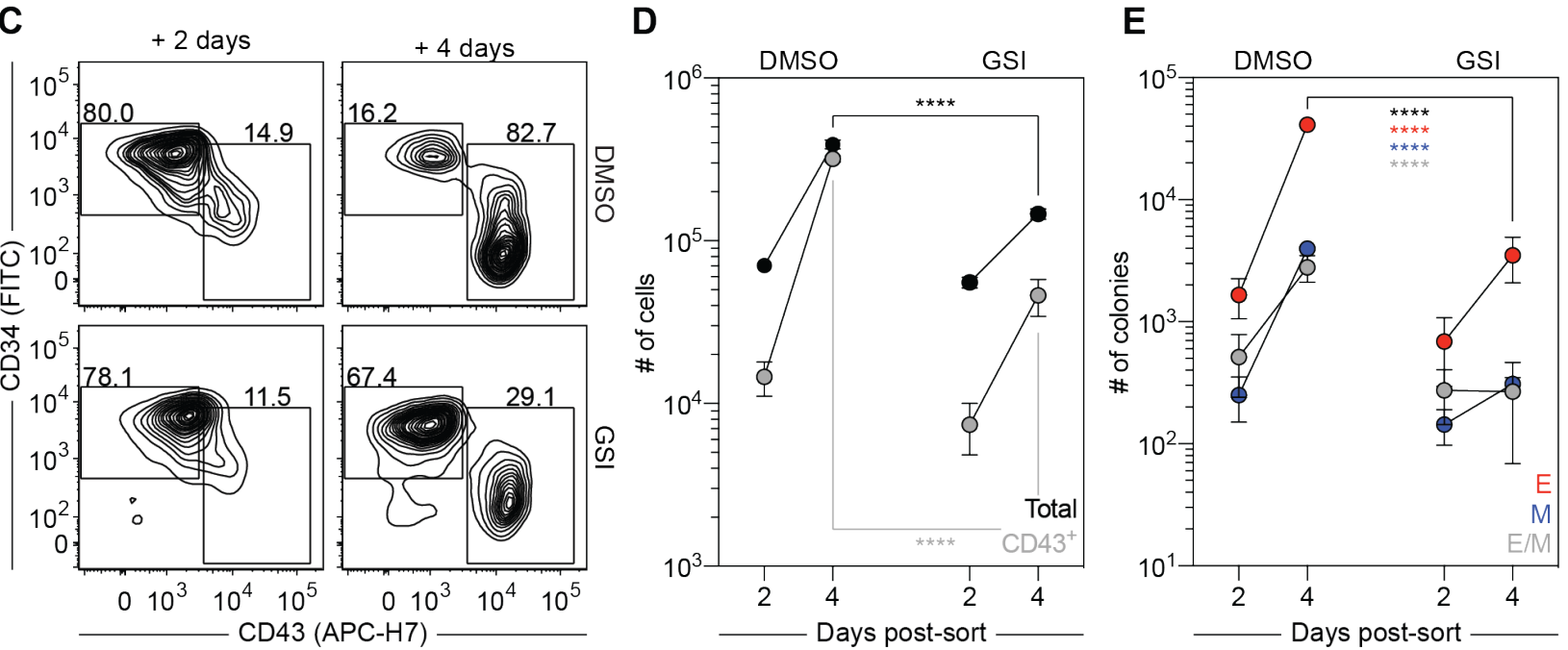

Figure 3.7 NOTCH signaling is required for the generation of the hematopoietic cells of the primitive hematopoietic program.

A. RT-qPCR analysis of NOTCH target gene expression over 4 days of monolayer culture of the day $5 \mathrm{KDR}^{+} \mathrm{CD} 34^{+}$cells in the presence or absence of the NOTCH inhibitor, GSI $(\mathrm{n}=3)$. ANOVA. ${ }^{* *} P<0.01$ versus the stage-matched sample.

B. Representative phase-contrast images of populations generated from the day $5 \mathrm{KDR}^{+} \mathrm{CD} 34^{+}$ cells cultured in the presence or absence of the NOTCH inhibitor, GSI.

C. Representative flow cytometric analysis of CD34 and CD43 expression of populations generated from day $5 \mathrm{KDR}^{+} \mathrm{CD} 34^{+}$cells cultured in monolayer in the presence or absence of the NOTCH inhibitor, GSI.

D. Quantification of the number of total and $\mathrm{CD}^{2} 3^{+}$cells generated from 20,000 day 5 $\mathrm{KDR}^{+} \mathrm{CD} 34^{+}$cells cultured in the presence or absence of the NOTCH inhibitor, GSI $(\mathrm{n}=3)$. ANOVA. $* * * * P<0.0001$ versus the stage-matched population.

E. Number of colony-forming progenitors generated from 20,000 day $5 \mathrm{KDR}^{+} \mathrm{CD} 34^{+}$cells cultured for the indicated time in the presence or absence of the NOTCH inhibitor, GSI ( $\mathrm{n}=$ 3). ANOVA. $* * * * P<0.0001$. Colonies: $\mathrm{E}=$ erythroid (red), $\mathrm{M}=$ myeloid (blue) and $\mathrm{E} / \mathrm{M}=$ mixed erythro-myeloid (grey). 


\subsubsection{The primitive hematopoietic program requires MYB}

As studies in the mouse have demonstrated that Myb is required for the development of the EMP, LMP and definitive hematopoietic programs, but not the primitive hematopoietic program (Allen et al., 1999; Mucenski et al., 1991; Mukouyama et al., 1999; Tober et al., 2008), I was next interested to determine whether this transcription factor played a similar role in human hematopoietic development. To address this, I generated a MYB-deficient hPSC line by inserting a series of translational stop codons into the fifth exon of the gene using CRISPR-Cas9 genome editing (Figure 3.8a and 3.8b). I identified one hESC clone that lacked wild type MYB $(0.022 \%$ of transfected cells) and expanded it for further study. The $\mathrm{MYB}^{-/-} \mathrm{hESCs}$ had a normal karyocyte and expressed the expected hPSC markers, SSEA-5, TRA1-60 and E-cadherin and lacked the expression of the endoderm marker, CXCR4 (Figure 3.8c and 3.8d).

The lack of MYB did not affect the induction of $\mathrm{KDR}^{+} \mathrm{CD} 235 \mathrm{a} / \mathrm{b}^{+}$mesoderm or the total cellularity of the cultures at day 4 of differentiation (Figure 3.9). Similarly, the total cellularity and emergence of the $\mathrm{KDR}^{+} \mathrm{CD} 34^{+} \mathrm{KIT}^{+} \mathrm{CD} 144^{+} \mathrm{CD} 31^{+}$population was unaffected in the absence of MYB at day 5 of differentiation (Figure 3.10). To determine whether MYB is required to generate the hematopoietic cells of the primitive hematopoietic program, I isolated the $\mathrm{MYB}^{+/+}$and $\mathrm{MYB}^{-/-}$ $\mathrm{KDR}^{+} \mathrm{CD} 34^{+}$cells on day 5 of differentiation and cultured them as monolayers in the presence of VEGF, FGF2 and hematopoietic cytokines. As indicated earlier, this culture format supports the development of an adherent layer of cells from wild type $\mathrm{KDR}^{+} \mathrm{CD} 34^{+}$cells that undergo EHT to give rise to non-adherent hematopoietic cells that rapidly expand in number. While the MYBdeficient cells also formed an adherent monolayer that gave rise to some non-adherent cells, their numbers did not increase to the same extent as observed in cultures of wild type cells (Figure 3.11a to $3.11 \mathrm{c})$. Flow cytometric analyses after 4 days of culture also revealed differences between the $\mathrm{MYB}^{+/+}$and $\mathrm{MYB}^{-/-}$hematopoietic populations. Whereas, the majority of CD $43^{+}$cells in the wild type cultures did not express CD34 or CD45, most of the $\mathrm{MYB}^{-/-} \mathrm{CD} 43^{+}$cells were $\mathrm{CD} 34^{+}$and of these, approximately half also expressed CD45 (Figure 3.11d and 3.11e). Analyses of colonyforming progenitor potential demonstrated that the pattern of erythroid, myeloid and erythromyeloid colonies observed in wild type cultures was disrupted in the absence of MYB. The MYBdeficient cells only gave rise to macrophage colonies, in contrast to wild type cells that gave rise to erythroid, macrophage and mast cell colonies (Figure 3.11f). Collectively, these data demonstrate that while MYB is not required for the development of the primitive macrophage 
lineage, the human primitive erythroid and mast cell lineages are MYB-dependent. The requirement for MYB in the human primitive hematopoietic program is clearly different than what has been observed in the mouse, where the development of all of the hematopoietic lineages of the primitive hematopoietic program are spared in its absence. 


\section{A

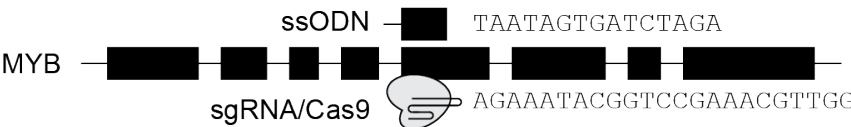

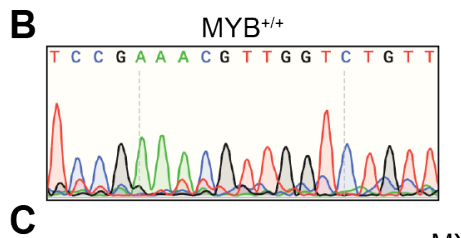

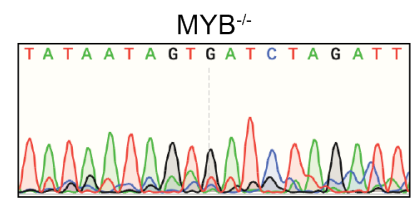

MYB
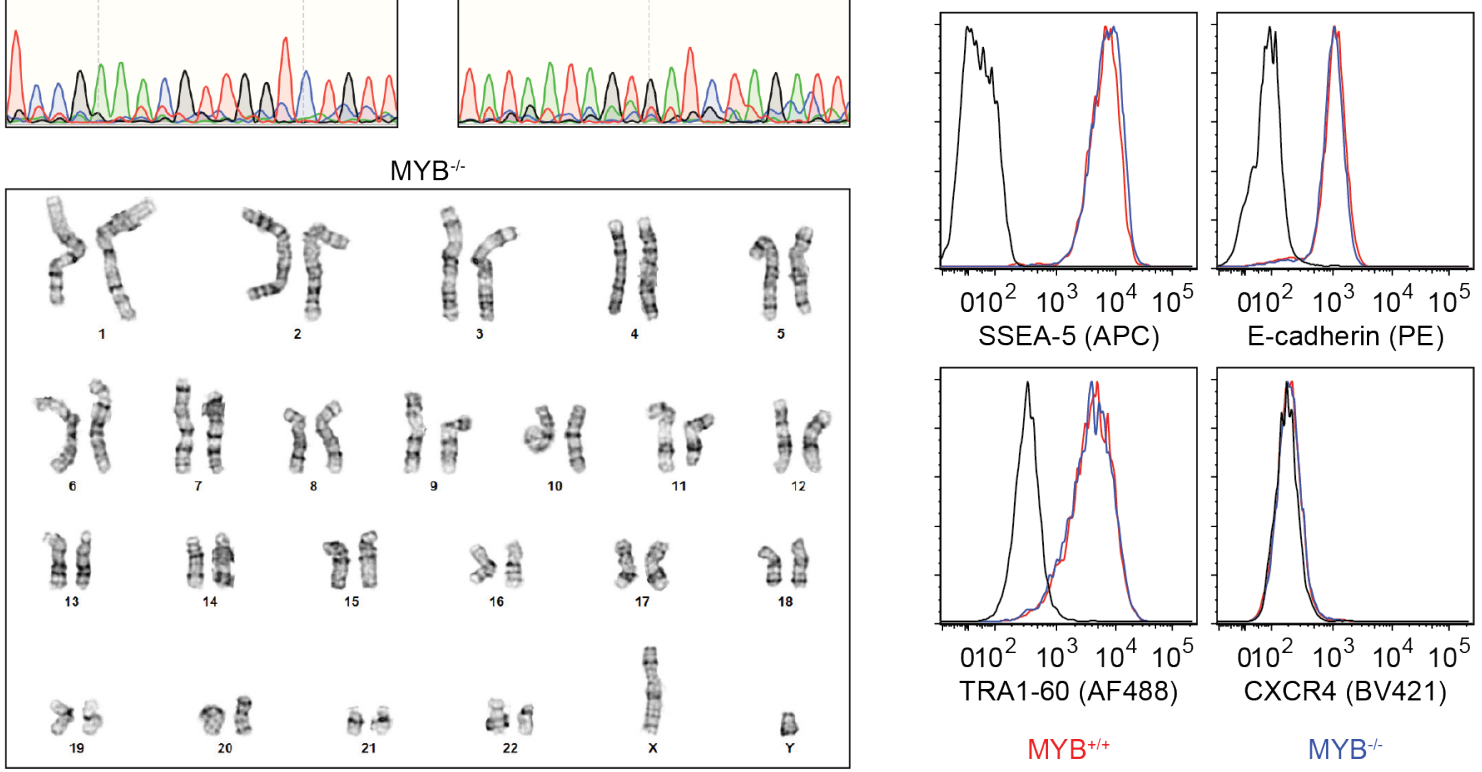

Figure 3.8 Generation and characterization of $\mathrm{MYB}^{-/-} \mathrm{H} 1 \mathrm{hESCs}$.

A. Schematic of the CRISPR-Cas9 strategy that was used to target the $5^{\text {th }}$ exon of MYB.

B. Sanger sequencing chromatogram of the targeted site in the $5^{\text {th }}$ exon of MYB showing the insertion of the ssODN.

C. Karyotypic analysis of the $\mathrm{MYB}^{-/-} \mathrm{H} 1 \mathrm{hESC}$ line.

D. Representative flow cytometric analysis of SSEA-5, TRA1-60, E-cadherin and CXCR4 on wild type and MYB-deficient hESCs. Black line: unstained cells. 

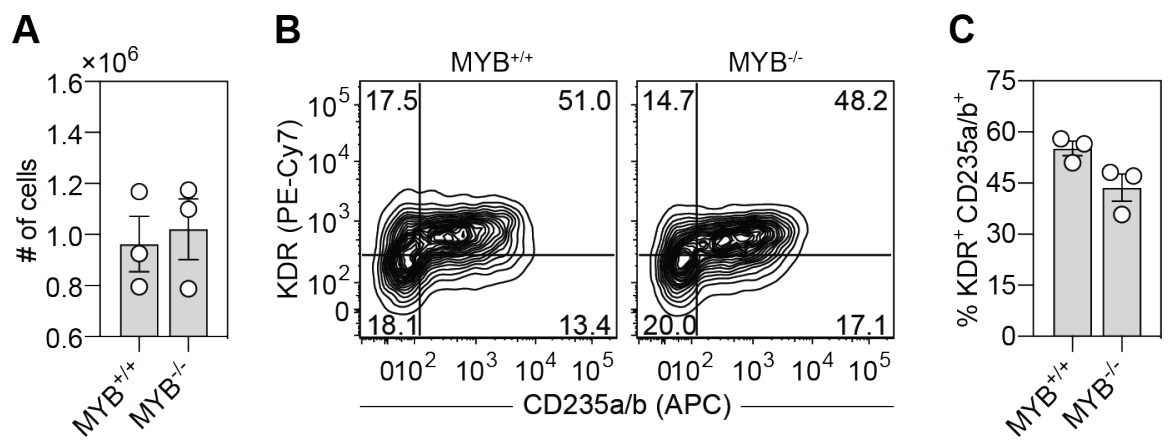

Figure 3.9 MYB is not required for the generation of $\mathrm{KDR}^{+} \mathrm{CD} 235 \mathrm{a}^{+}$mesoderm.

A. Quantification of cell numbers in wild type and MYB-deficient populations on day 4 of differentiation $(n=3)$. t-test. not significant.

B. Representative flow cytometric analysis of KDR and CD235a/b expression on wild type and MYB-deficient populations on day 4 of differentiation.

C. Quantification of the proportion of $\mathrm{KDR}^{+} \mathrm{CD} 235 \mathrm{a} / \mathrm{b}^{+}$cells on day 4 of differentiation $(\mathrm{n}=3)$. t-test. not significant. 

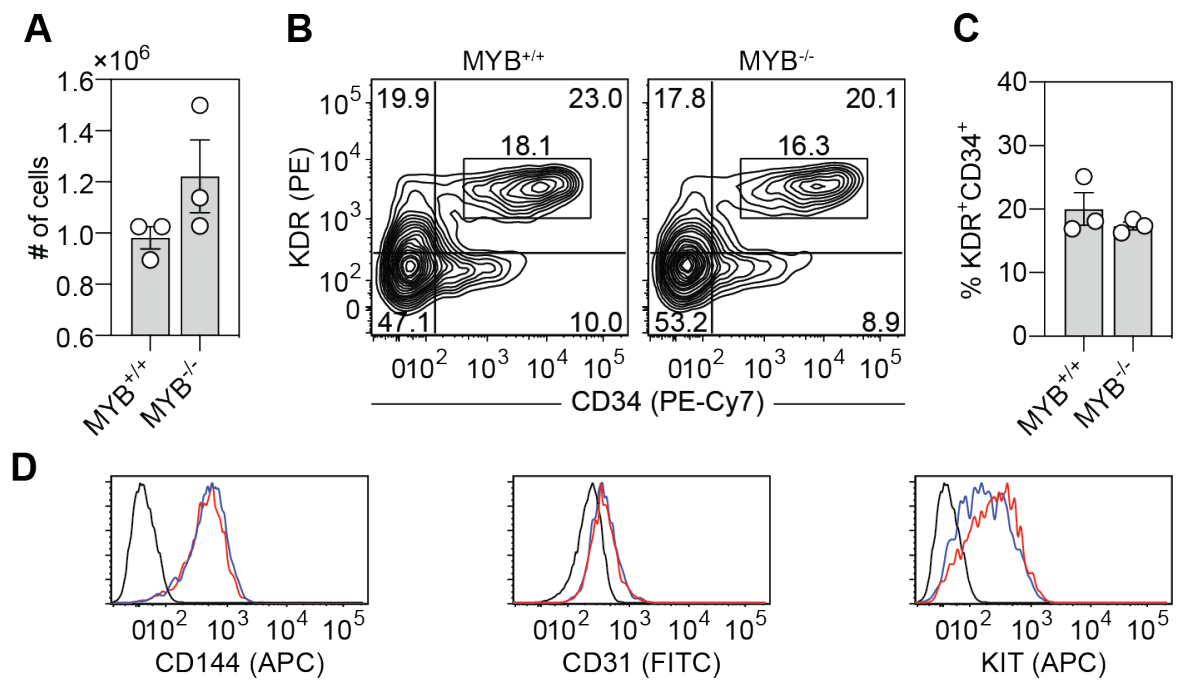

$\mathrm{MYB}^{+/+} \quad \mathrm{MYB}^{-/-}$

Figure $3.10 \mathrm{MYB}$ is not required for the generation of $\mathrm{KDR}^{+} \mathrm{CD} 34^{+}$cells.

A. Quantification of cell numbers in wild type and MYB-deficient cultures on day 5 of differentiation $(n=3)$. $t$-test. not significant.

B. Representative flow cytometric analysis of KDR and CD34 expression on wild type and MYBdeficient populations on day 5 of differentiation.

C. Quantification of the proportion of $\mathrm{KDR}^{+} \mathrm{CD} 34^{+}$cells in wild type and MYB-deficient cultures on day 5 of differentiation $(n=3)$. t-test. not significant.

D. Representative flow cytometric analysis of KIT, CD144 and CD31 expression on the day 5 $\mathrm{KDR}^{+} \mathrm{CD} 34^{+}$wild type and MYB-deficient populations. Black line: unstained control. 
A

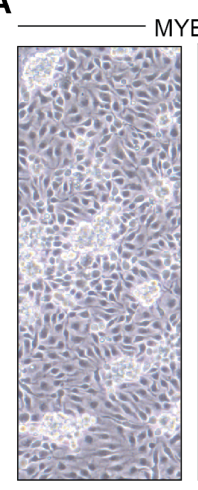

+2 days

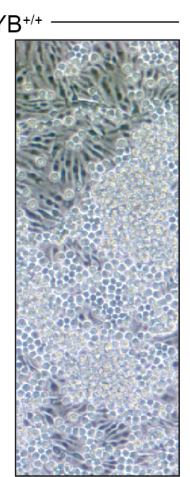

+4 days

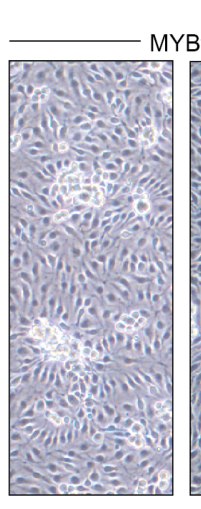

+2 days

-sort
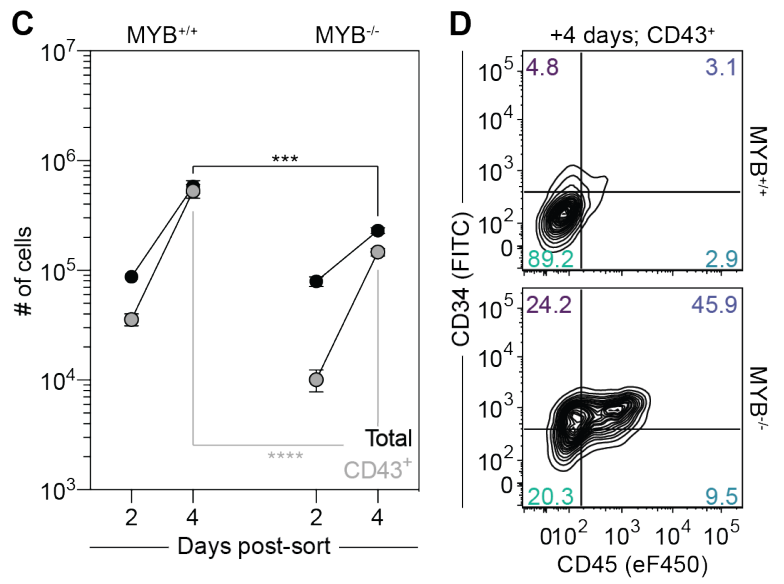

$\mathbf{E}$

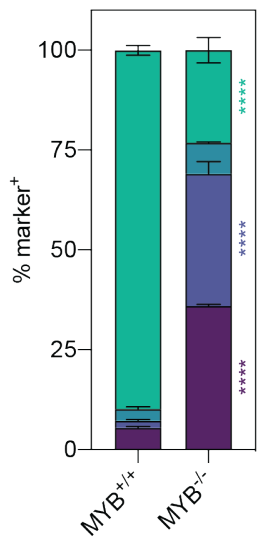

B

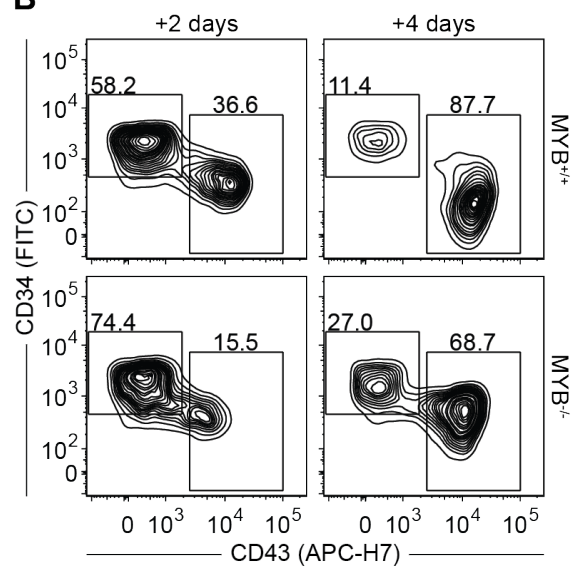

$\mathbf{F}$

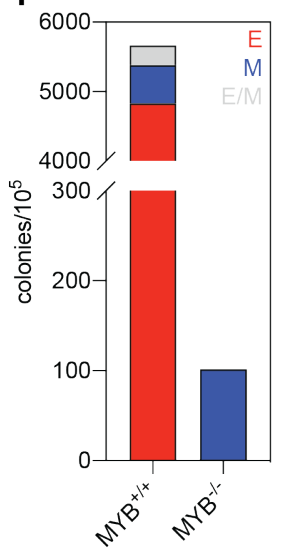

G

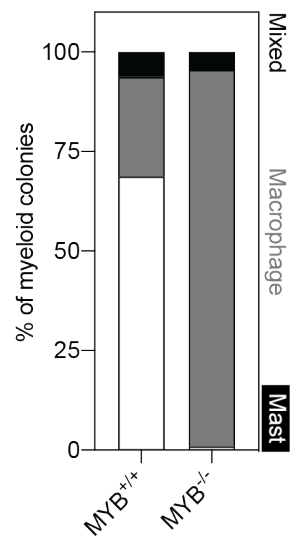

Figure 3.11 MYB is required for the generation of the hematopoietic cells of the primitive hematopoietic program.

A. Representative phase-contrast images of monolayer cultures derived from the day 5 $\mathrm{KDR}^{+} \mathrm{CD} 34^{+}$cells. Scale bar $=50 \mu \mathrm{m}$.

B. Representative flow cytometric analysis of CD34 and CD43 expression on populations generated from day $5 \mathrm{KDR}^{+} \mathrm{CD} 34^{+}$cells in monolayer culture.

C. Quantification of the number of total and $\mathrm{CD}^{2} 3^{+}$cells generated from 20,000 day 5 $\mathrm{KDR}^{+} \mathrm{CD} 34^{+}$cells in monolayer cultures $(\mathrm{n}=3)$. ANOVA. ${ }^{* * *} P<0.001$ and $* * * * P<0.0001$ versus the stage-matched population from $\mathrm{MYB}^{+/+}$cultures.

D. Representative flow cytometric analysis of CD34 and CD45 expression on the CD43 ${ }^{+}$ population following 4 days of monolayer culture of the day $5 \mathrm{KDR}^{+} \mathrm{CD} 34^{+}$cells

E. Quantification of the proportion of the different CD34 and CD45 populations shown in $\mathrm{D}(\mathrm{n}=$ 3). ANOVA. ${ }^{* * * *} P<0.0001$ versus the matched population from $\mathrm{MYB}^{+/+}$cultures. The colours of each bar corresponds to the frequency of the indicated population shown in D.

F. Colony-forming progenitor frequency in day $9 \mathrm{MYB}^{+/+}$and $\mathrm{MYB}^{-/-} \mathrm{EBs}(\mathrm{n}=1)$. Colonies: $\mathrm{E}=$ erythroid (red), $\mathrm{M}=$ myeloid (blue) and $\mathrm{E} / \mathrm{M}=$ mixed erythro-myeloid (grey).

G. Distribution of myeloid progenitors in day $9 \mathrm{MYB}^{+/+}$and $\mathrm{MYB}^{-/-} \mathrm{EBs}(\mathrm{n}=1)$. 


\subsubsection{Identification of a second hemogenic endothelial cell population with broader hematopoietic lineage potential}

In addition to the emergent $\mathrm{CD} 43^{+}$hematopoietic cells on day 6 of differentiation, the EBs also contained a $\mathrm{CD} 34^{+} \mathrm{CD} 43^{-} \mathrm{CD} 45^{-}$population that co-expressed the HEC/endothelial cell markers KDR, KIT, CD144 and CD31 (Figure 3.12a and 3.12b). RT-qPCR analyses also showed that the $\mathrm{CD} 34^{+} \mathrm{CD}^{-} 3^{-}$population expressed the HEC transcription factors $R U N X 1$ and $S C L / T A L 1$ (Figure 3.12c) suggesting the presence of HECs.

To characterize the hematopoietic potential of the day $6 \mathrm{CD} 43^{+}$and $\mathrm{CD} 34^{+} \mathrm{CD} 43^{-}$populations, I isolated them by FACS and cultured the cells as aggregates in the presence of VEGF, FGF2 and hematopoietic cytokines for 6 days (Figure 3.13a). The $\mathrm{CD} 43^{+}$population expanded over the initial 3 days of culture and was stable thereafter (Figure 3.13b and 3.13c). Although the number of hematopoietic cells progressively increased over the course of the culture, the number of colonyforming progenitors decreased between days 3 to 6 of culture (Figure 3.13d) suggestive of the exhaustion of the primitive hematopoietic program. In contrast, cultures generated from the $\mathrm{CD} 34^{+} \mathrm{CD}^{-} 3^{-}$population gave rise to a small $\mathrm{CD} 3^{+}$population following 1 day of culture that increased in size over the 6 days of culture (Figure 3.13b and 3.13c). The total number of colonyforming progenitors also increased over this time (Figure 3.13d). Analyses of colony subtypes showed a dominance of the erythroid lineage at all stages in the CD43 ${ }^{+}$-derived population. In contrast, the $\mathrm{CD} 34^{+} \mathrm{CD} 43^{-}$-derived population showed a more balanced distribution of erythroid and myeloid progenitors between days 1 and 3 of culture. The erythroid lineage dominated at 6 days of culture (Figure 3.13e), possibility reflecting a bias in our culture conditions or the high demand for erythropoiesis in the developing fetus. The pattern of lineage development from the $\mathrm{CD} 34^{+} \mathrm{CD}^{-} 3^{-}$population between days 1 and 3 of culture is consistent with the distribution of erythroid and myeloid colony-forming progenitors in the mouse E9.5 $\mathrm{CD}^{+} 1^{+} \mathrm{Kit}^{+} \mathrm{CD} 16 / 32^{+} \mathrm{EMP}$ population (McGrath et al., 2015). Analyses of colony morphologies showed that the CD43 ${ }^{+}$ population largely gives rise to small colonies of erythroblasts. In contrast, larger erythroid colonies were abundant between days 1 and 3 of culture in populations derived from the $\mathrm{CD}^{+} 4^{+} \mathrm{CD} 43^{-}$cells. Smaller colonies dominated after 6 days of culture (Figure 3.13f) and likely originated from the 


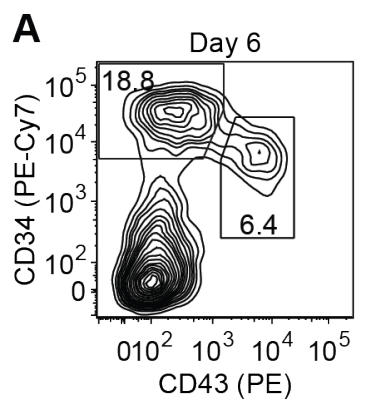

B
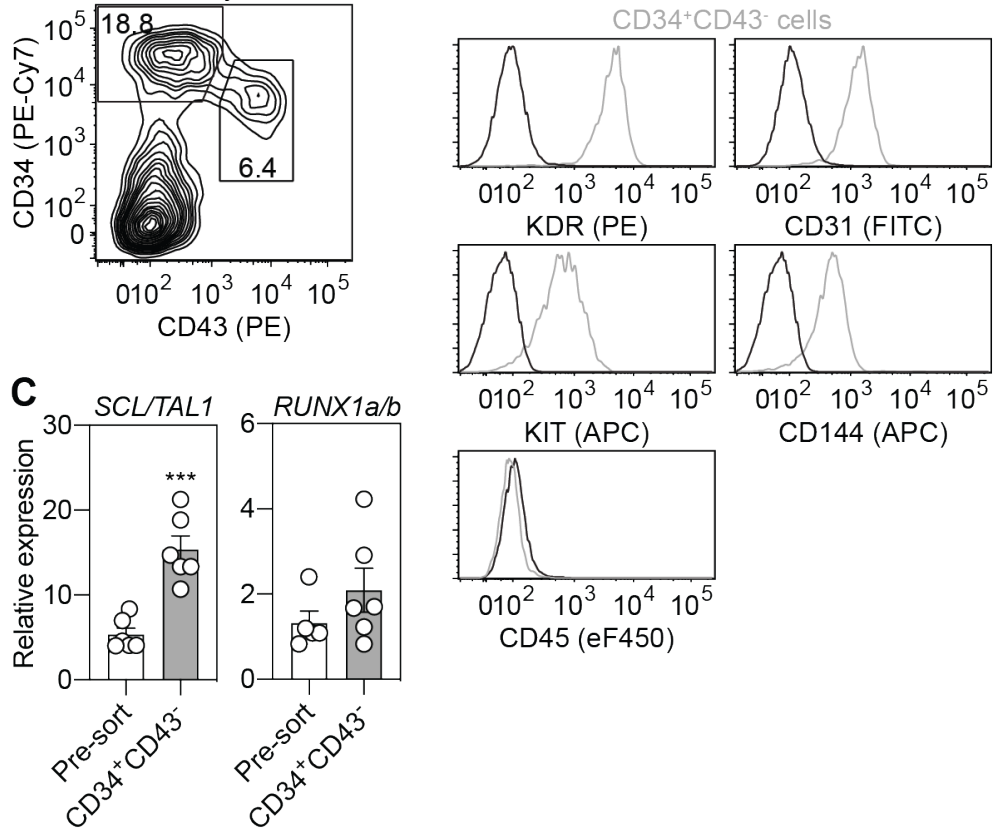

Figure 3.12 Characterization of $\mathrm{CD} 34^{+} \mathrm{CD} 43^{-}$cells at day 6 of differentiation.

A. Representative flow cytometric analysis of CD34 and CD43 expression on day 6 of differentiation.

B. Representative flow cytometric analysis of KDR, KIT, CD144, CD31 and CD45 expression on the day $6 \mathrm{CD} 34^{+} \mathrm{CD} 43^{-}$population (gray). Black line: unstained cells.

C. RT-qPCR analysis of $S C L / T A L 1$ and $R U N X 1 a / b$ expression in the day $6 \mathrm{CD}^{-} 4^{+} \mathrm{CD}^{-} 3^{-}$and presort populations $(\mathrm{n}=6)$. t-test. $* * * P<0.001$. 

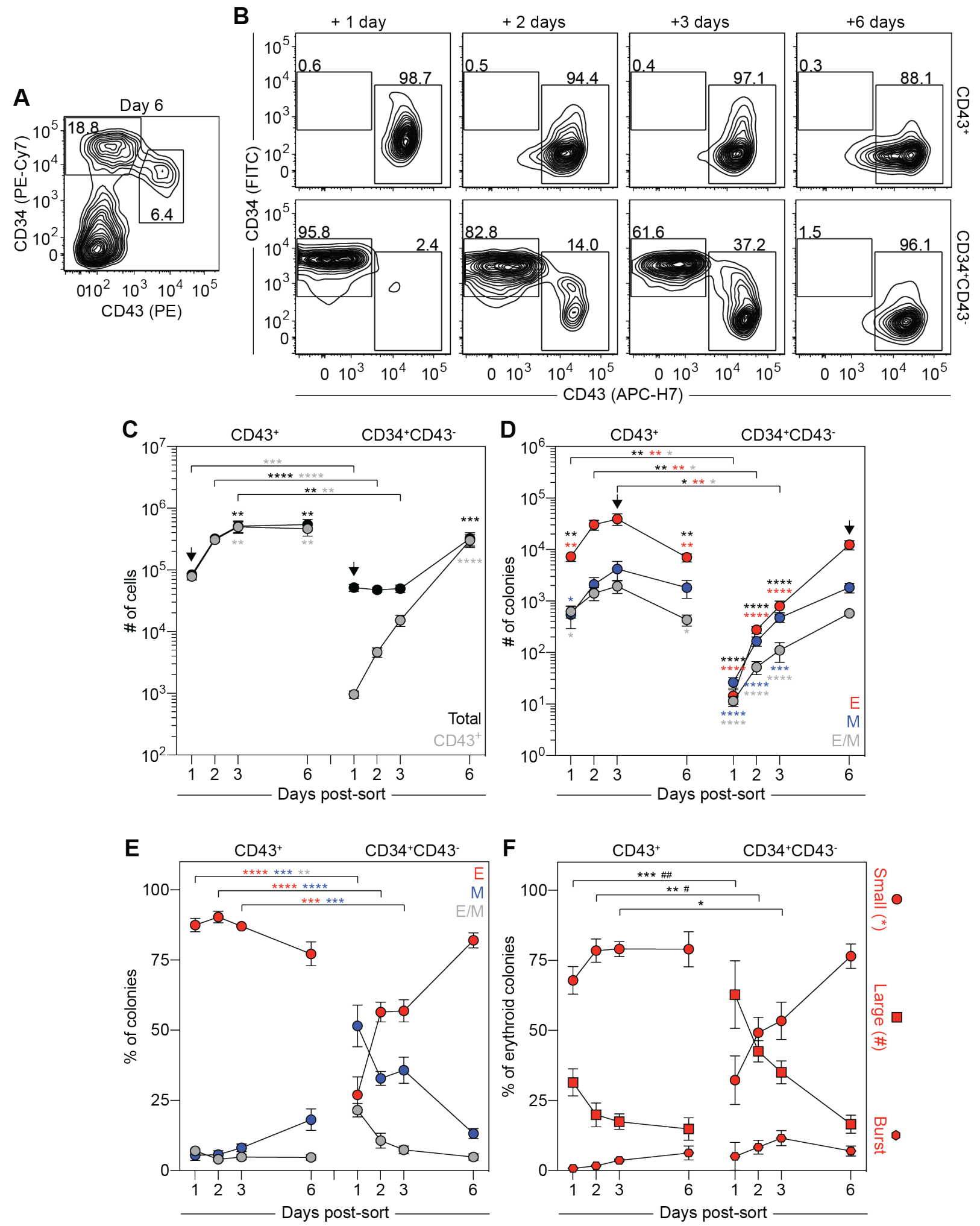
Figure 3.13 Separation of two hematopoietic programs at day 6 of differentiation based on CD34 and CD43 expression.

A. Gating strategy for the FACS based isolation of the day $6 \mathrm{CD}^{+} 3^{+}$and $\mathrm{CD} 34^{+} \mathrm{CD} 43^{-}$ populations.

B. Representative flow cytometric analysis of CD34 and CD43 expression on the populations generated from $\mathrm{CD}_{4} 3^{+}$and $\mathrm{CD} 34^{+} \mathrm{CD} 43^{-}$cells isolated on day 6 of differentiation.

C. Quantification of the number of total and $\mathrm{CD} 43^{+}$cells generated from 62,500 day $6 \mathrm{CD} 43^{+}$or $\mathrm{CD} 34^{+} \mathrm{CD} 43^{-}$isolated cells $(\mathrm{n}=5)$. $\mathrm{t}$-test and ANOVA. $* * P<0.01,{ }^{* * *} P<0.001$ and $* * * * P$ $<0.0001$ versus the stage-matched sample or versus after 1 day of culture within the same sample, as indicated.

D. Colony-forming progenitor numbers in populations generated from 62,500 day $6 \mathrm{CD}^{2} 3^{+}$or CD34 ${ }^{+}$CD $43-$ cells $(\mathrm{n}=5)$. t-test and ANOVA. $* P<0.05, * * P<0.01$ and $* * * * P<0.0001$ versus the stage-matched sample or versus after 1 day of culture within the same sample, as indicated (black = all colonies). Colonies: $\mathrm{E}=$ erythroid (red), $\mathrm{M}=$ myeloid (blue) and $\mathrm{E} / \mathrm{M}=$ mixed erythro-myeloid (grey).

E. Distribution of lineages observed in D. ANOVA. ${ }^{* *} P<0.01,{ }^{* * *} P<0.001$ and $* * * * P<$ 0.0001 versus the stage-matched sample. Colonies: $\mathrm{E}=$ erythroid (red), $\mathrm{M}=$ myeloid (blue) and $\mathrm{E} / \mathrm{M}=$ mixed erythro-myeloid (grey).

F. Distribution of erythroid progenitors observed in D. ANOVA. $* P<0.05, * * P<0.01$ and $* * * P$ $<0.001$, and $\# P<0.05$ and $\# \# P<0.01$ versus small and large erythroid colony morphologies, respectively. 
erythroid colony-forming progenitors that gave rise to large colonies at earlier stages of culture. The erythroid colony morphologies generated from the $\mathrm{CD} 43^{+}$and $\mathrm{CD} 34^{+} \mathrm{CD} 43^{-}$populations are consistent with erythroid colonies that are produced by the primitive and EMP programs, respectively (Palis et al., 1999).

As CD45 is expressed on the hematopoietic progenitors of the EMP, LMP and definitive hematopoietic programs, but not those of the primitive hematopoietic program (Ferkowicz et al., 2003), I next monitored its expression on cells generated from the day $6 \mathrm{CD}_{4} 3^{+}$and CD $34^{+} \mathrm{CD} 43^{-}$ populations. Cultures derived from the $\mathrm{CD} 43^{+}$population contained a small $\mathrm{CD} 45^{+}$population following 6 days of culture, which likely represent the maturing myeloid cells of the primitive hematopoietic program. In contrast, cultures generated from the $\mathrm{CD} 34^{+} \mathrm{CD} 43^{-}$population contained a large number of $\mathrm{CD} 45^{+}$cells that may represent emerging EMPs (Figure 3.14a to $3.14 \mathrm{c})$. To test this, I isolated the following fractions derived from the $\mathrm{CD} 34^{+} \mathrm{CD} 43^{-}$population by FACS after 5 days of culture: $\mathrm{CD} 34^{+} \mathrm{CD} 45^{+} \mathrm{KIT}^{+}, \mathrm{CD} 34^{+} \mathrm{CD} 45^{+} \mathrm{KIT}^{-}, \mathrm{CD}_{4} 4^{-} \mathrm{CD} 45^{+}$and $\mathrm{CD} 34^{-}$ $\mathrm{CD}^{-}$cells and assayed them for colony-forming progenitor potential (Figure 3.14d). KIT was included as it is expressed on mouse EMPs (Ferkowicz et al., 2003; McGrath et al., 2015). The CD34-CD45- population was highly enriched in small erythroid colony-forming progenitors that likely represent a combination of contaminating primitive erythroid progenitors and EMP-derived erythroid progenitors that have matured to the stage at which they have downregulated CD45. The majority of the progenitors in the $\mathrm{CD} 45^{+}$populations were of the mast cell and macrophage lineages (Figure 3.14e and 3.14f). I also detected granulocyte progenitors in these cultures, a lineage generated from EMPs, but not primitive progenitors (Palis et al., 1999). Erythroid lineage cells were also detected in this population, however they were largely associated with mixed erythro-myeloid colonies. RT-qPCR of these colonies showed higher levels of expression of the fetal $\beta$ globin, $H B G$ relative to colonies generated from the day $6 \mathrm{CD} 43^{+}$population (Figure $3.14 \mathrm{~g}$ ) indicating that the $\mathrm{CD} 34^{+} \mathrm{CD} 45^{+} \mathrm{KIT}^{+}$-derived erythroid cells are representative of the human EMP lineage. Taken together, these findings identify the emergence of distinct populations of CD34 ${ }^{+}$ cells that display developmental potential indicative of the human primitive and EMP programs. 


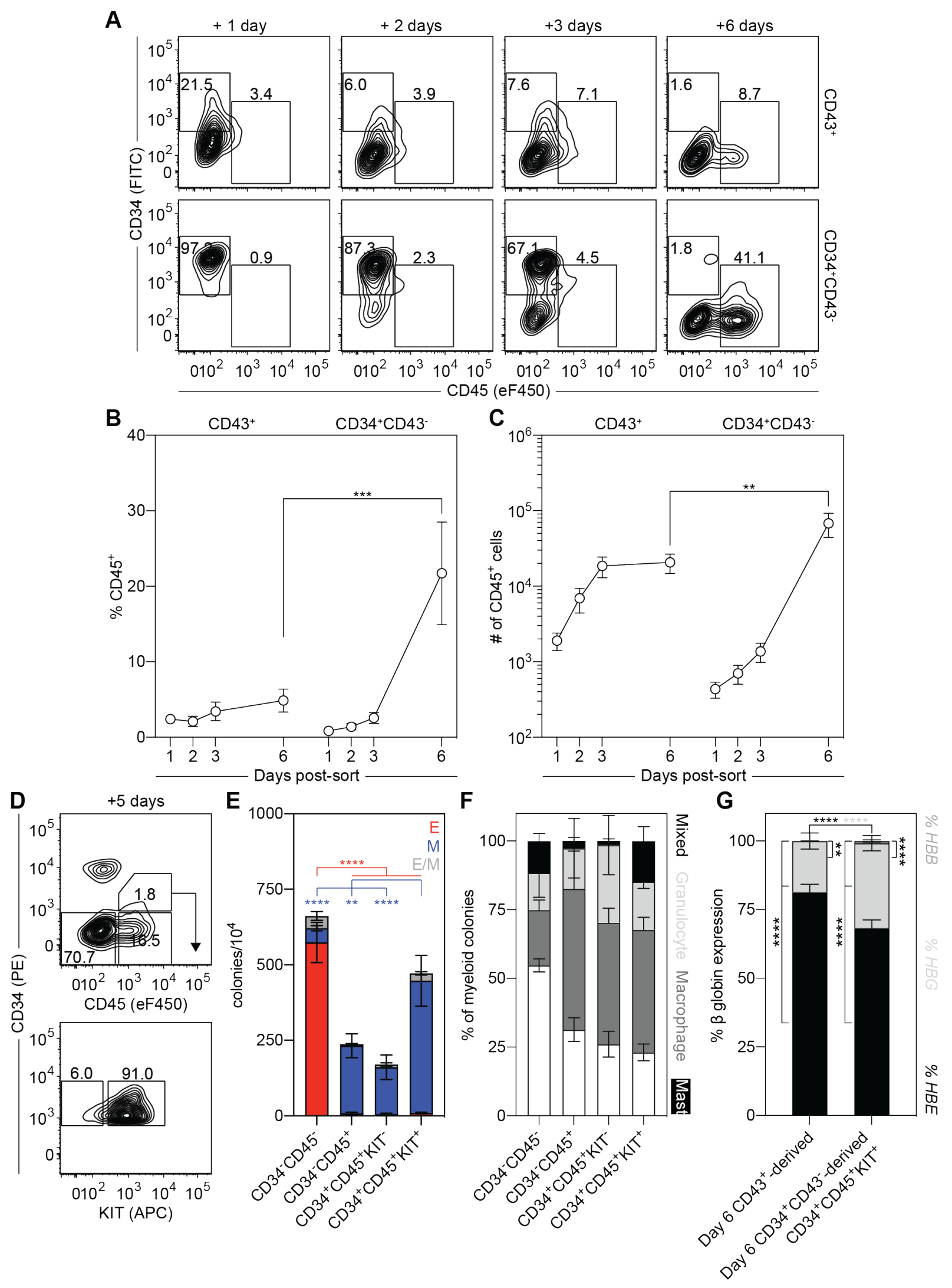


Figure $3.14 \mathrm{CD} 34^{+} \mathrm{CD} 43^{-} \mathrm{HECs}$ give rise to $\mathrm{CD} 45^{+}$hematopoietic progenitors.

A. Representative flow cytometric analysis of CD34 and CD45 expression on populations generated from the day $6 \mathrm{CD} 43^{+}$and $\mathrm{CD} 34^{+} \mathrm{CD} 43^{-}$cells.

B. Quantification of the proportion of $\mathrm{CD} 45^{+}$cells over 6 days in the populations generated from the day $6 \mathrm{CD} 3^{+}$and $\mathrm{CD} 34^{+} \mathrm{CD} 43^{-}$cells $(\mathrm{n}=5)$. ANOVA. $* * * P<0.001$ versus the stagematched population.

C. Quantification of the number of $\mathrm{CD} 45^{+}$cells over in the populations generated from the day 6 $\mathrm{CD} 3^{+}$and $\mathrm{CD} 4^{+} \mathrm{CD} 43^{-}$cells $(\mathrm{n}=5)$. ANOVA. ${ }^{* *} P<0.01$ versus the stage-matched population.

D. Gating strategy used for FACS-based isolation of the CD34 and CD45 populations generated from the day $6 \mathrm{CD} 34^{+} \mathrm{CD} 43^{-}$cells. Cells were cultured as aggregates for 5 days.

E. Colony-forming progenitor frequency in the isolated CD34 and CD45 populations shown in D $(\mathrm{n}=3)$. ANOVA. $* P<0.05$ and $* * * * P<0.0001$ versus the indicated lineage and population. Colonies: $\mathrm{E}=$ erythroid (red), $\mathrm{M}=$ myeloid (blue) and $\mathrm{E} / \mathrm{M}=$ mixed erythro-myeloid (grey).

F. Distribution of myeloid progenitors observed in E.

G. RT-qPCR analysis of the percentage of $H B E, H B G$ and $H B B \beta$ globin expression of erythroid colonies derived from the day $6 \mathrm{CD} 43^{+}$population after 6 days of culture and $\mathrm{CD} 34^{+} \mathrm{CD} 45^{+} \mathrm{KIT}^{+}$cells generated after 5 days of culture of the day $6 \mathrm{CD} 34^{+} \mathrm{CD} 43^{-}$population $(\mathrm{n}=4-12) . * * P<0.01$ and $* * * * P<0.0001$ versus the indicated $\beta$ globin or sample. 
Given the emergence of a program with EMP-like potential, I was next interested in determining if the $\mathrm{CD} 34^{+} \mathrm{CD} 43^{-}$population also contained $\mathrm{T}$ lymphoid progenitors. To test this, I cultured both the day $6 \mathrm{CD} 34^{+} \mathrm{CD} 43^{-}$and $\mathrm{CD} 43^{+}$populations with OP9-DL4 cells that have been engineered to provide levels of NOTCH signaling required for human T cell development (Mohtashami et al., 2013). As shown in Figure $3.15 \mathrm{a}$, the $\mathrm{CD} 34^{+} \mathrm{CD} 43^{-}$, but not $\mathrm{CD}^{+} 3^{+}$population generated $\mathrm{CD} 45^{+} \mathrm{CD} 56^{-} \mathrm{CD}^{+} \mathrm{CD} 8^{+} \mathrm{T}$ lymphoid progenitors following 1 month of culture with OP9-DL4 cells. $\alpha \beta$ or $\gamma \delta \mathrm{TCR}^{+}$lymphocytes were detected following an additional 10 days of culture (Figure $3.15 \mathrm{~b}$ and 3.15c). To confirm that the $\mathrm{T}$ lymphoid lineage was generated from the $\mathrm{KDR}^{+} \mathrm{CD} 235 \mathrm{a} / \mathrm{b}^{+}$yolk sac-like mesoderm, I next isolated this population and cultured the cells as aggregates for 3 days and then seeded them onto OP9-DL4 cells (Figure 3.16a and 3.16b). During the 3 day aggregation culture, the $\mathrm{KDR}^{+} \mathrm{CD} 235 \mathrm{a} / \mathrm{b}^{+}$cells generated a population that consisted of approximately $85 \% \mathrm{CD} 34^{+} \mathrm{CD} 43^{-}$cells. Analyses after 1 month of culture revealed the presence of $\mathrm{CD}^{+} \mathrm{CD} 8^{+} \mathrm{T}$ lymphoid progenitors demonstrating that $\mathrm{KDR}^{+} \mathrm{CD} 235 \mathrm{a} / \mathrm{b}^{+}$mesoderm does give rise to the $\mathrm{T}$ lymphoid lineage (Figure 3.16c). To determine if this $\mathrm{T}$ lymphoid lineage is different from that generated from cord blood hematopoietic progenitors, I compared the surface marker expression of the $\mathrm{CD} 7^{+}$progenitors after 12 days of culture, as this represents one of the earliest stages of lymphoid commitment. Analyses of the cord blood-derived cells showed that CD5 was expressed on the differentiating $\mathrm{CD} 7{ }^{+} \mathrm{CD} 34^{+} \mathrm{T}$ lymphoid progenitor population. In contrast, the hPSC-derived $\mathrm{CD}^{+} \mathrm{CD} 34^{+}$cells were $\mathrm{CD}^{-}$(Figure 3.17). Collectively, these findings show that $\mathrm{KDR}^{+} \mathrm{CD} 235 \mathrm{a} / \mathrm{b}^{+}$mesoderm induced with BMP4, FGF2 and Activin A contains primitive, EMP and $\mathrm{T}$ lymphoid potential, largely reflecting the hematopoietic potential of the mouse yolk sac. These studies also provide evidence that the development of the hPSC-derived T lymphocytes may differ from those generated from definitive cord blood progenitors. 
A

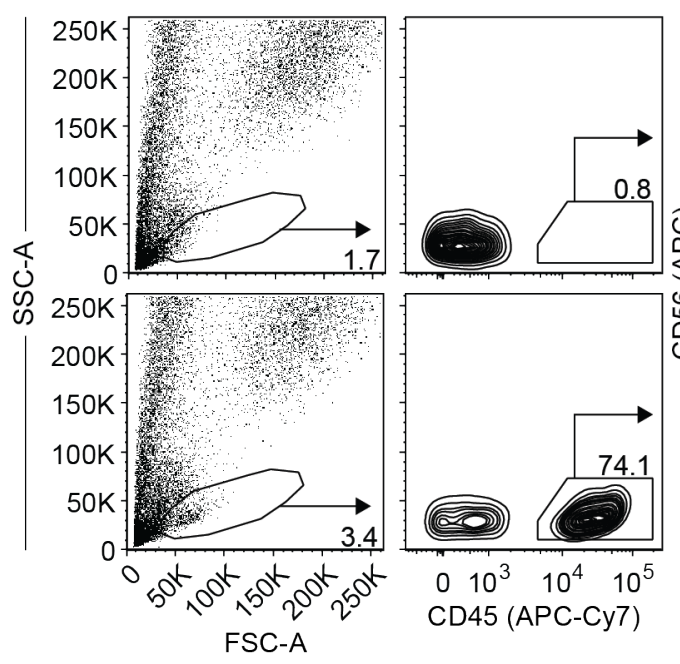

B

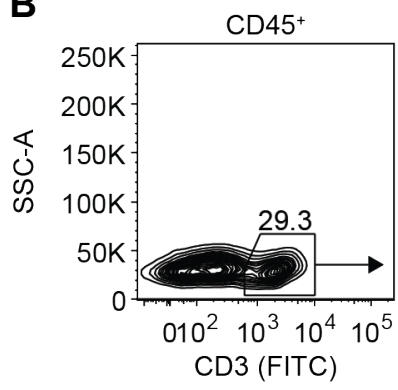

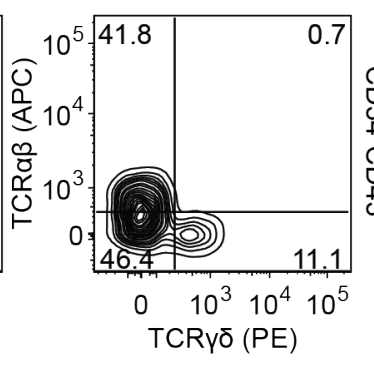
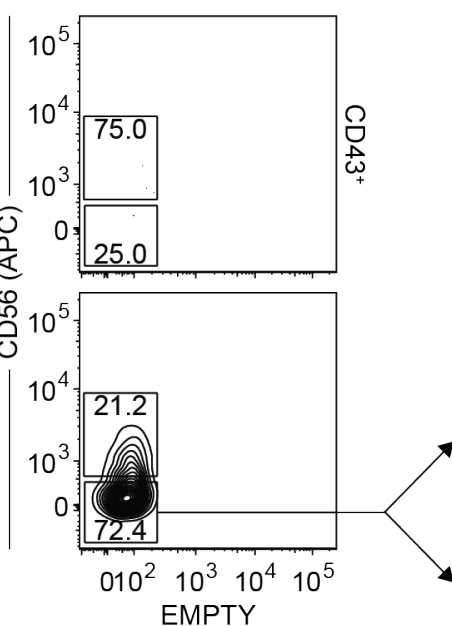

C

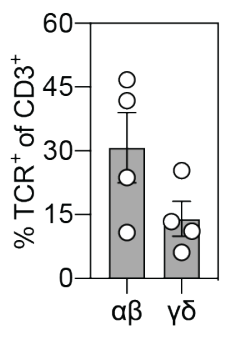

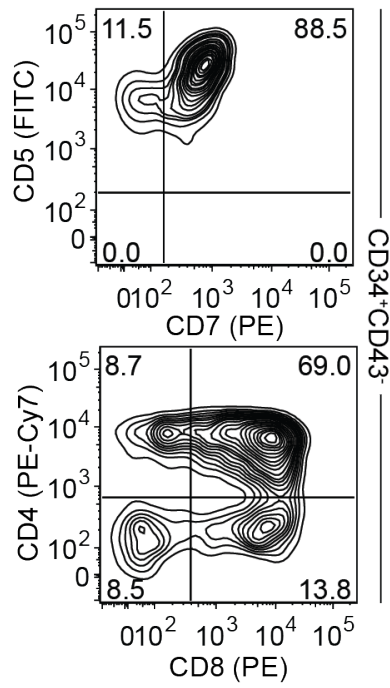

Figure 3.15 CD34+CD43- HECs have T lymphoid potential.

A. Representative flow cytometric analysis of CD45, CD56, CD5, CD7, CD4 and CD8 expression on cells derived from the day $6 \mathrm{CD} 3^{+}$or $\mathrm{CD} 34^{+} \mathrm{CD} 43^{-}$populations following 30 days of culture with OP9-DL4 cells.

B. Representative flow cytometric analysis of CD3, TCR $\alpha \beta$ and TCR $\gamma \delta$ expression on the CD45 cells generated from the day $6 \mathrm{CD} 34^{+} \mathrm{CD} 43^{-}$population co-cultured with OP9-DL4 cells for 40 days.

C. Quantification of the proportion of $\mathrm{CD} 3^{+} \mathrm{TCR} \alpha \beta^{+}$and $\mathrm{CD} 3^{+} \mathrm{TCR} \gamma \delta^{+}$cells in the day $40 \mathrm{CD} 45^{+}$ populations described in $B(n=4)$. t-test. not significant. 

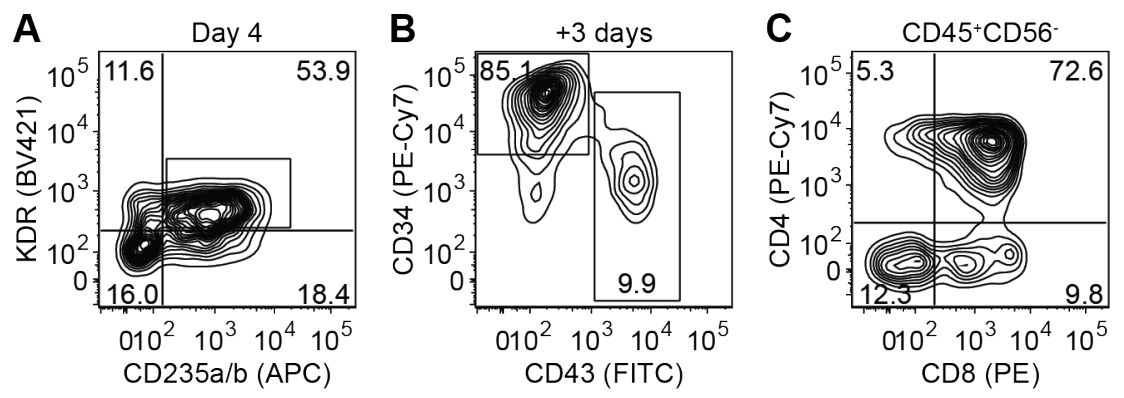

Figure 3.16 KDR ${ }^{+} \mathrm{CD} 235 \mathrm{a} / \mathrm{b}^{+}$mesoderm gives rise to the $\mathrm{T}$ lymphoid lineage.

A. Gating strategy used for the FACS-based isolation of the $\mathrm{KDR}^{+} \mathrm{CD} 235 \mathrm{a} / \mathrm{b}^{+}$population on day 4 of differentiation.

B. Representative flow cytometric analysis of CD34 and CD43 expression on the day 4 $\mathrm{KDR}^{+} \mathrm{CD} 235 \mathrm{a} / \mathrm{b}^{+}$-derived population following 3 days of culture.

C. Representative flow cytometric analysis of CD4 and CD8 expression on the $\mathrm{CD}^{4} 5^{+} \mathrm{CD} 56^{-}$ population generated from the $\mathrm{CD} 34^{+} \mathrm{CD} 43^{-}$cells (shown in $\mathrm{B}$ ) following culture for 30 days with OP9-DL4 cells. 


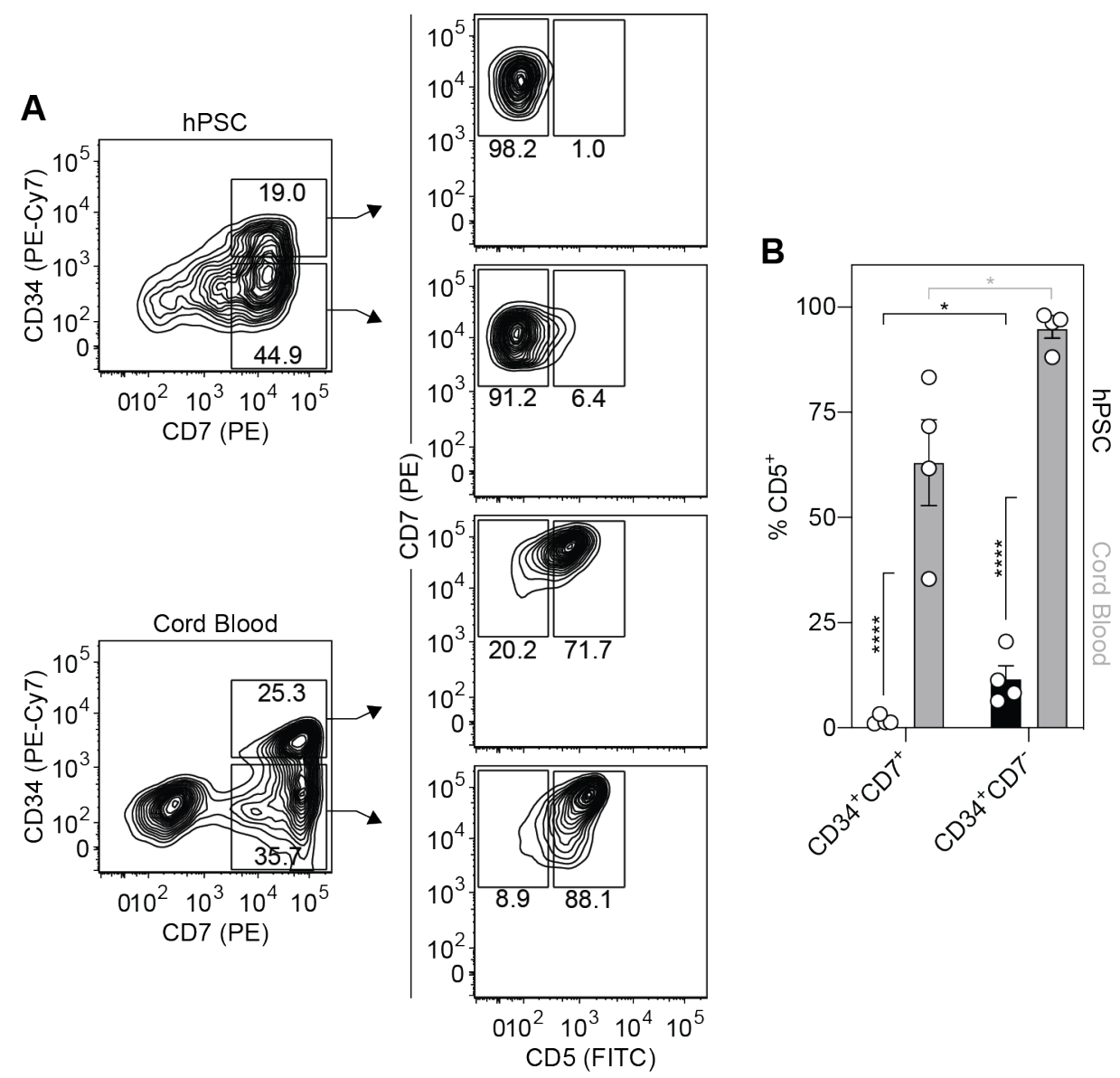

Figure 3.17 Analyses of T lymphoid progenitors.

A. Representative flow cytometric analysis of CD34, CD7 and CD7 expression on the CD45 populations generated from $\mathrm{hPSC}$-derived $\mathrm{CD} 34^{+} \mathrm{CD} 43^{-}$cells or $\mathrm{CD} 34^{+}$cord blood following 12 days of culture with OP9-DL4 cells.

B. Quantification of the proportion of $\mathrm{CD}^{+}$cells in either the $\mathrm{CD} 34^{+} \mathrm{CD} 7^{+}$and $\mathrm{CD} 344^{-} \mathrm{CD} 7^{+}$ $\mathrm{CD} 45^{+}$populations generated from $\mathrm{hPSC}$-derived $\mathrm{CD} 34^{+} \mathrm{CD} 43^{-}$cells or $\mathrm{CD} 34^{+}$cord blood following 12 days of culture with OP9-DL4 cells $(\mathrm{n}=4)$. ANOVA. $* P<0.05$ and $* * * * P<$ 0.0001 versus the indicated population. 


\subsubsection{The erythro-myeloid and T lymphoid progenitors are generated from multipotent hematopoietic progenitors}

The close temporal association between the EMP and LMP programs in the mouse and the demonstration that the hPSC-derived $\mathrm{CD} 34^{+} \mathrm{CD} 43^{-} \mathrm{HEC}$ population contained erythroid, myeloid and lymphoid potentials raised the possibility that these lineages develop from a common multipotent progenitor. To search for this progenitor, I first cultured the day $6 \mathrm{CD} 34^{+} \mathrm{CD} 43^{-}$cells with OP9-DL4 cells for 5 days to initiate EHT and then analyzed the developing hematopoietic populations for the presence of cells that express markers of hematopoietic progenitors, including CD34, CD45, CD90 and CD7. From these analyses, I was able to identify distinct $\mathrm{CD} 34^{+} \mathrm{CD} 45^{+} \mathrm{CD} 90^{+} \mathrm{CD} 7^{-}$and $\mathrm{CD} 34^{+} \mathrm{CD} 45^{+} \mathrm{CD} 90^{-} \mathrm{CD}^{+}$populations (Figure 3.18a). Although CD90 and CD7 were expressed in day $6 \mathrm{CD} 43^{+}$primitive hematopoietic population, these cells did not express CD45 (Figure 3.18b). I next performed limiting dilution analyses to determine if the frequency of lymphoid progenitors in either the $\mathrm{CD} 90^{+} \mathrm{CD} 7^{-}$or $\mathrm{CD} 90^{-} \mathrm{CD} 7^{+} \mathrm{CD} 34^{+} \mathrm{CD} 45^{+}$ subpopulations was high enough to carry out single cell clonal analyses. These studies showed that the frequencies of $\mathrm{NK}\left(\mathrm{CD}^{+} \mathrm{CD}^{-}: 1: 16\right.$ and $\left.\mathrm{CD}^{-} \mathrm{CD}^{+}: 1: 82\right)$ and $\mathrm{T}$ lymphoid $\left(\mathrm{CD}^{-} 0^{+} \mathrm{CD}^{-}\right.$: $1: 26$ and $\mathrm{CD}^{-} \mathrm{CD}^{+}: 1: 73$ ) progenitors were higher in the $\mathrm{CD} 90^{+} \mathrm{CD}^{+}$population (Figure 3.19a). I also measured the proliferative potential of the two $\mathrm{CD} 34^{+} \mathrm{CD} 45^{+}$subpopulations by culturing the cells with HUVEC-E4ORF1 endothelial cells, which have been shown to support the expansion of hematopoietic progenitors (Seandel et al., 2008). Consistent with its higher hematopoietic progenitor frequency, the $\mathrm{CD} 90^{+} \mathrm{CD}^{-}$population gave rise to significantly more total $\mathrm{CD}_{4} 5^{+}$and more $\mathrm{CD} 34^{+} \mathrm{CD} 45^{+}$cells than the $\mathrm{CD} 90^{-} \mathrm{CD} 7^{+}$population after 4 days of coculture (Figure 3.19b and 3.19c). These data suggested that the $\mathrm{CD} 90^{+} \mathrm{CD}^{-}$population is suitable target population for clonal analyses and that HUVEC-E4ORF1 endothelial cells are able to efficiently expand hematopoietic progenitors. Although the HUVEC-E4ORF1 cells supported hematopoietic expansion, NK cell and T lymphoid potentials were lost from both $\mathrm{CD} 34^{+} \mathrm{CD} 45^{+}$ subpopulations following 4 days of culture (data not shown). By comparison, culture with OP9DL4 cells for 8 days produced fewer CD $45^{+}$hematopoietic cells (Figure 3.19d), but allowed for progenitors with lymphoid potential to be maintained. To determine if short-term culture with OP9-DL4 cells is supportive of multilineage hematopoiesis, I cultured 25 $\mathrm{CD} 34^{+} \mathrm{CD} 45^{+} \mathrm{CD} 90^{+} \mathrm{CD} 7^{-}$cells on OP9-DL4 stroma for 4 days then assayed $30 \%$ of the culture for erythroid and myeloid progenitors in methylcellulose and the remaining cells for lymphoid potential by co-culture with fresh OP9-DL4 cells. Of the 12 wells tested, 6 (50\%) gave rise to 

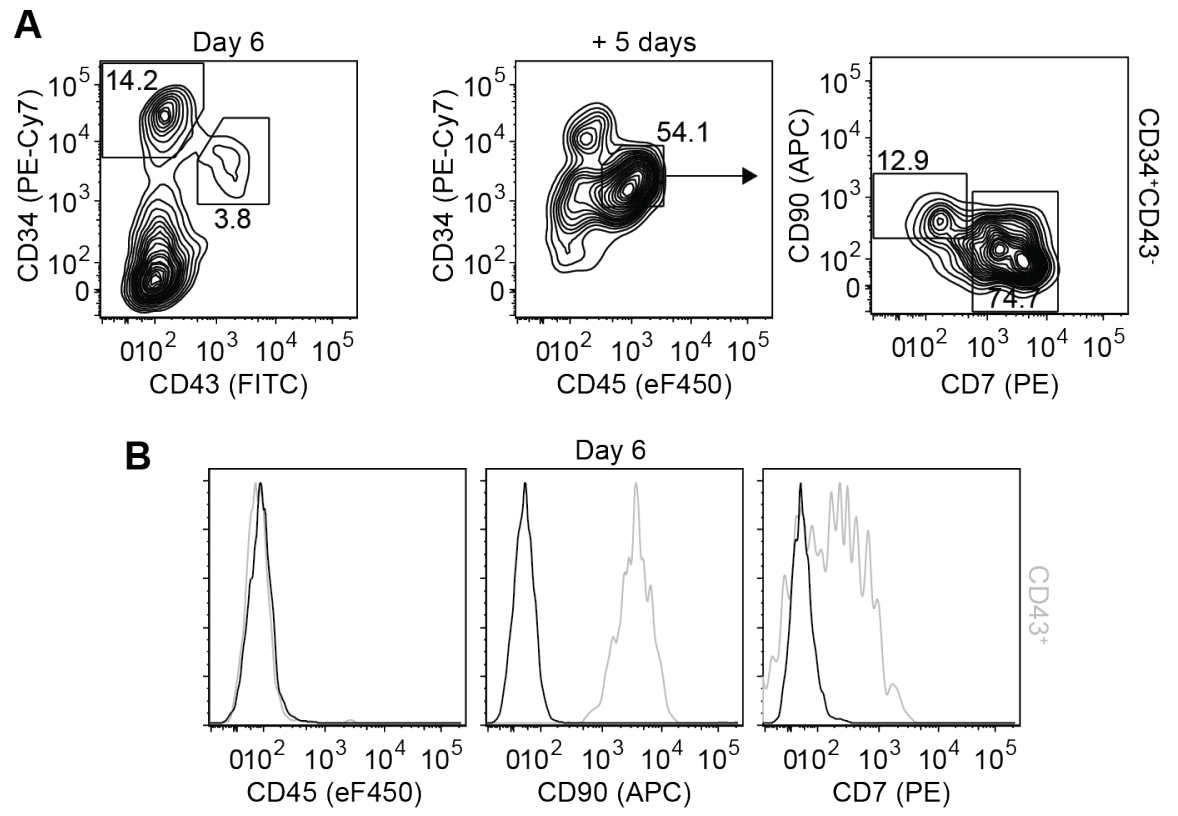

Figure 3.18 Characterization of the day $6 \mathrm{CD} 43^{+}$and $\mathrm{CD} 34^{+} \mathrm{CD} 43^{-} \mathrm{HEC}$-derived hematopoietic cells.

A. Representative flow cytometric analysis of CD34, CD45, CD90 and CD7 expression on the day $6 \mathrm{CD} 34^{+} \mathrm{CD} 43^{-}$-derived population generated following 5 days of culture with OP9-DL4 cells.

B. Representative flow cytometric analysis of CD45, CD90 and CD7 expression on the day 6 $\mathrm{CD}_{4} 3^{+}$population. Black line: unstained cells. 
A
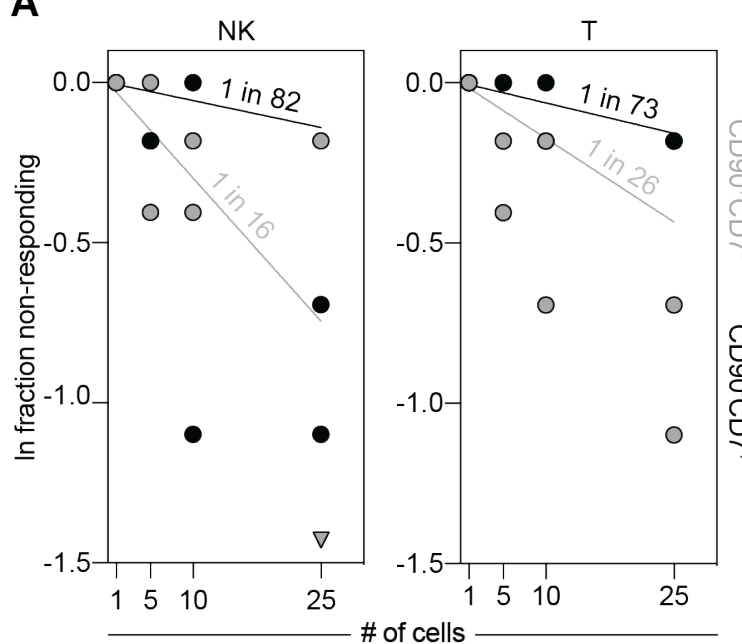

D

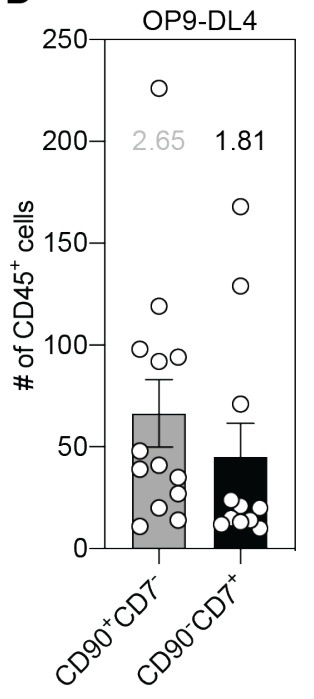

E

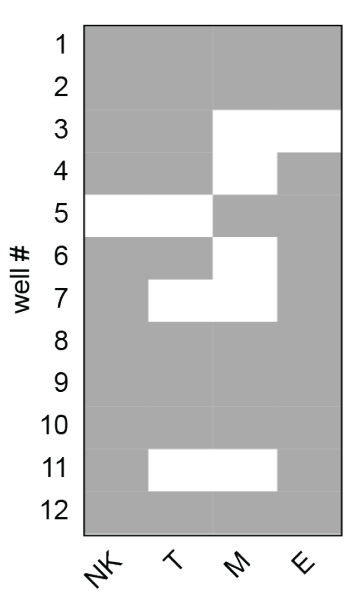

B $1000 \frac{\text { HUVEC-E4ORF1 }}{\mid \begin{array}{lll}1886 & 8.23\end{array}} 400 \frac{\text { HUVEC-E4ORF1 }}{7.01}$
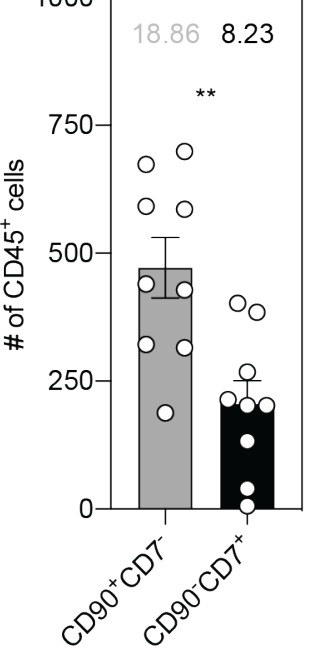

F

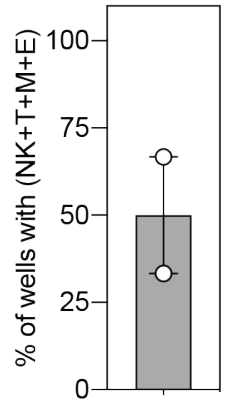


Figure 3.19 The expression of CD90 and absence of CD7 marks a CD34 ${ }^{+} \mathrm{CD} 45^{+}$ hematopoietic progenitor population with multilineage potential.

A. Limiting dilution analysis of $\mathrm{NK}$ cell and $\mathrm{T}$ lymphoid progenitor frequency of the $\mathrm{CD} 34^{+} \mathrm{CD} 45^{+} \mathrm{CD} 90^{+} \mathrm{CD} 7{ }^{-}$and $\mathrm{CD} 34^{+} \mathrm{CD} 45^{+} \mathrm{CD} 90^{-} \mathrm{CD} 7^{+}$populations $(\mathrm{n}=2)$.

B. Quantification of the number of $\mathrm{CD} 45^{+}$cells generated from $25 \mathrm{CD} 34^{+} \mathrm{CD} 45^{+} \mathrm{CD} 90^{+} \mathrm{CD} 7^{-}$or $\mathrm{CD} 34^{+} \mathrm{CD} 45^{+} \mathrm{CD} 90^{-} \mathrm{CD} 7^{+}$cells following 4 days of culture with HUVEC-E4ORF1 cells. The numbers within the graph indicate the average fold change in cell number $(\mathrm{n}=9)$. t-test. ${ }^{* *} P$ $<0.01$.

C. Quantification of the number of $\mathrm{CD} 34^{+} \mathrm{CD} 45^{+}$cells generated from 25 $\mathrm{CD} 34^{+} \mathrm{CD} 45^{+} \mathrm{CD} 90^{+} \mathrm{CD} 7{ }^{-}$or $\mathrm{CD} 34^{+} \mathrm{CD} 45^{+} \mathrm{CD} 90^{-} \mathrm{CD} 7^{+}$cells following 4 days of culture with HUVEC-E4ORF1 cells. The numbers within the graph indicate the average fold change in cell number $(\mathrm{n}=9)$. t-test. $* * P<0.01$.

D. Quantification of the number of $\mathrm{CD} 45^{+}$cells generated from $25 \mathrm{CD} 34^{+} \mathrm{CD} 45^{+} \mathrm{CD} 90^{+} \mathrm{CD} 7$ or $\mathrm{CD} 34^{+} \mathrm{CD} 45^{+} \mathrm{CD} 90^{-} \mathrm{CD} 7^{+}$cells following 8 days of culture with OP9-DL4 cells. The numbers within the graph indicate the average fold change in cell number $(n=12)$. t-test. not significant.

E. Heatmap representing wells that gave rise to NK cell (NK), T lymphoid (T), myeloid (M) and erythroid (E) progeny. Grey: lineage positive.

F. Quantification of the proportion of wells that generated all 4 lineages shown in E. 
lymphoid, erythroid and myeloid progeny (Figure 3.19e and 3.19f). These data demonstrate that co-culture with OP9-DL4 cells for 4 days preserves the multilineage hematopoietic potential of the $\mathrm{CD} 34^{+} \mathrm{CD} 45^{+} \mathrm{CD} 90^{+} \mathrm{CD} 7{ }^{-}$population.

To determine whether the $\mathrm{CD} 34^{+} \mathrm{CD} 45^{+} \mathrm{CD} 90^{+} \mathrm{CD} 7^{-}$population contains multipotent hematopoietic progenitors, I deposited single cells on OP9-DL4 cells by FACS. After 4 days, the cultures were harvested and assayed for erythroid, myeloid and lymphoid potentials, as described above. From 637 sorted $\mathrm{CD} 34^{+} \mathrm{CD} 45^{+} \mathrm{CD} 90^{+} \mathrm{CD} 7^{-}$cells, 60 cells $(9.4 \%)$ produced a hematopoietic clone (Figure 3.20a). Of the 60 clones, 10 (16.7\%) contained NK cell, T lymphoid, erythroid and myeloid progeny (Figure $3.20 \mathrm{~b}$ and $3.20 \mathrm{c}$ ) demonstrating that multipotent hematopoietic progenitors are contained within the $\mathrm{CD} 34^{+} \mathrm{CD} 45^{+} \mathrm{CD} 90^{+} \mathrm{CD} 7^{-}$population. The remaining 50 clones were largely restricted to the lymphoid $(20 \%)$ or erythro-myeloid $(25 \%)$ fates. I next compared $\beta$ globin expression of the erythroid colonies generated from each clone to erythroid colonies derived from the primitive and definitive hematopoietic programs. The erythroid colonies generated from the $\mathrm{CD} 34^{+} \mathrm{CD} 45^{+} \mathrm{CD} 90^{+} \mathrm{CD} 7^{-}$progenitors expressed lower levels of the embryonic $\beta$ globin, $H B E$ and higher levels of the fetal $\beta$ globin, $H B G$ than colonies of primitive erythroid cells and higher levels of $H B E$ and lower levels of $H B G$ than colonies generated from hPSCderived definitive progenitors (Figure 3.20d). This intermediate pattern is consistent with the $\beta$ globin expression pattern observed in the EMP-derived erythroid cells in the mouse. Taken together, these data demonstrate that the day $6 \mathrm{CD} 34^{+} \mathrm{CD} 43^{-}$HECs give rise to multipotent hematopoietic progenitors and in doing so, define a human multilineage yolk sac program that displays erythroid, myeloid and lymphoid potential. Given these findings, I propose that the EMP and LMP terminology used to describe the hematopoietic programs in the mouse yolk sac be revised to the multipotent progenitor program (MPP) for the human yolk sac. 


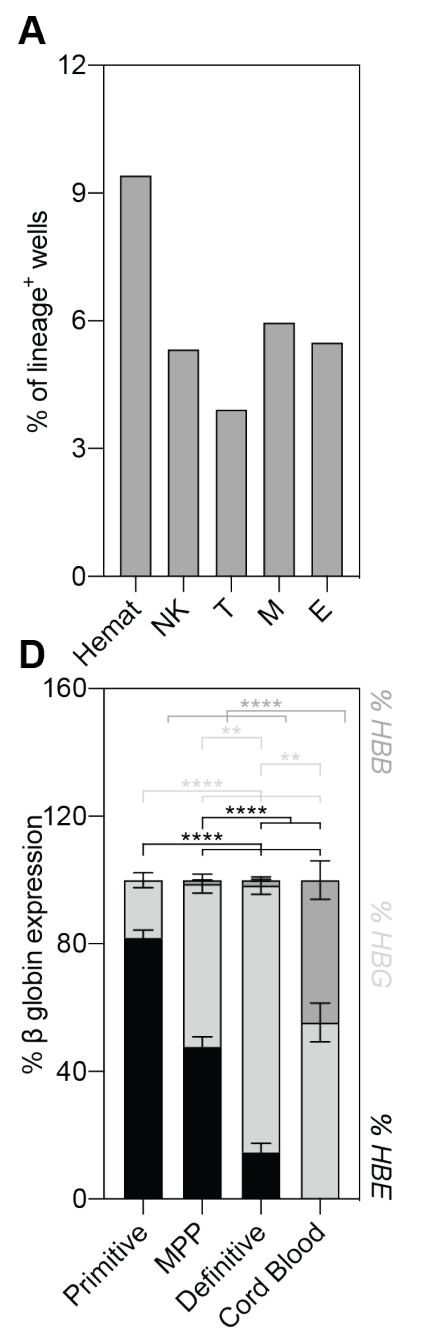

B
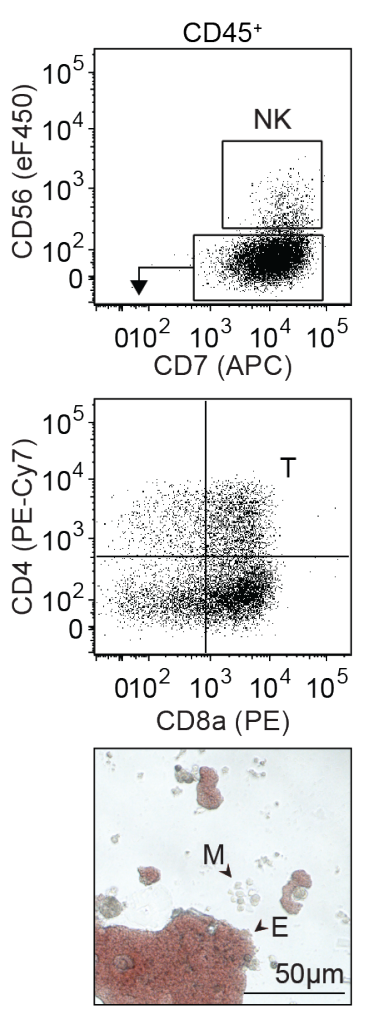

C

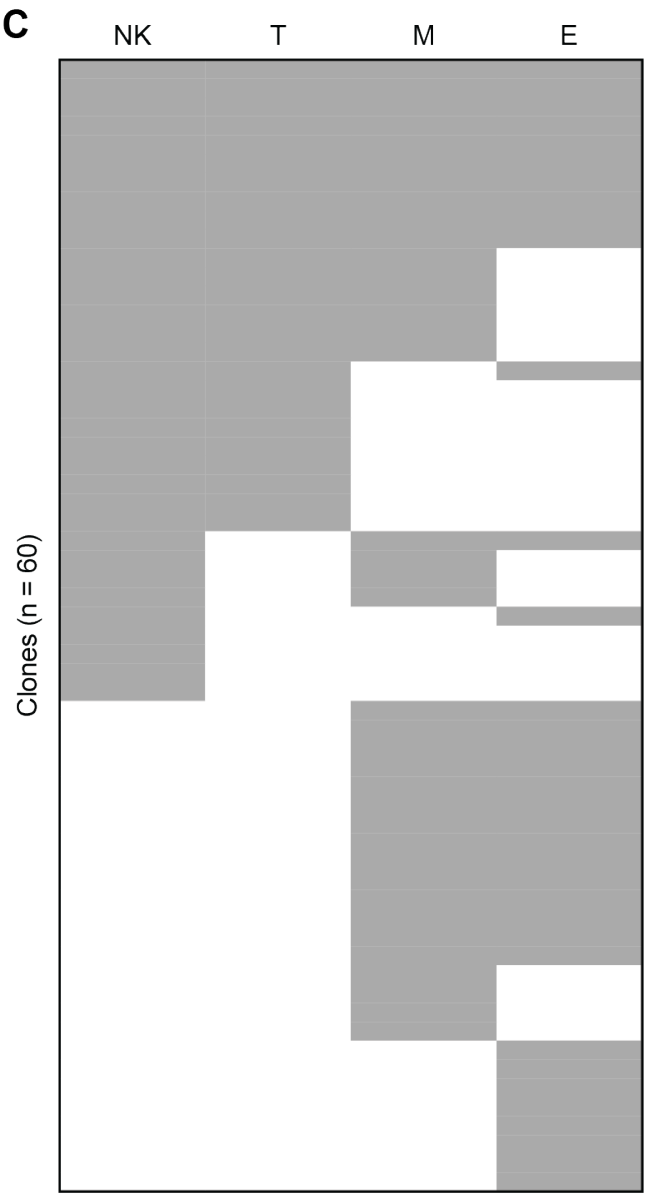

Figure 3.20 The $\mathrm{CD} 34^{+} \mathrm{CD} 45^{+} \mathrm{CD} 90^{+} \mathrm{CD} 7^{-}$population contains multipotent hematopoietic progenitors.

A. Quantification of the proportion of wells seeded with single $\mathrm{CD} 34^{+} \mathrm{CD} 45^{+} \mathrm{CD} 90^{+} \mathrm{CD} 7^{-}$cells that generated a hematopoietic clone.

B. Representative flow cytometric analysis of CD56, CD7, CD4 and CD8 expression and brightfield image identifying the NK cell (NK), T lymphoid (T), myeloid (M) and erythroid (E) lineages generated from a single $\mathrm{CD} 34^{+} \mathrm{CD} 45^{+} \mathrm{CD} 90^{+} \mathrm{CD} 7^{-}$cell.

C. Summary the NK cell (NK), T lymphoid (T), myeloid (M) and erythroid (E) lineage potential of all cells that gave rise to a hematopoietic clone $(n=60)$. Grey: lineage positive.

D. RT-qPCR analysis of the percentage of $H B E, H B G$ and $H B B \beta$ globin expression in erythroid colonies generated from the hPSC-derived primitive, MPP, definitive and cord blood progenitors $(\mathrm{n}=5-35)$. ANOVA. ${ }^{* *} P<0.01$ and $* * * * P<0.0001$ relative to the indicated sample. 


\subsubsection{Engraftment potential of multipotent hematopoietic progenitors}

Given the presence of multipotent hematopoietic progenitors in the $\mathrm{CD} 34^{+} \mathrm{CD} 45^{+} \mathrm{CD} 90^{+} \mathrm{CD} 7^{-}$ population, I next asked if it also contains engraftable progenitors equivalent to those identified in the E9.5 mouse yolk sac (Yoder and Hiatt, 1997; Yoder et al., 1997a; Yoder et al., 1997b). To test this, I transplanted between 4,000 and 9,000 hPSC-derived $\mathrm{CD} 34^{+} \mathrm{CD} 45^{+} \mathrm{CD} 90^{+} \mathrm{CD} 7{ }^{-}$cells into the livers of irradiated NOD.Cg-Prkd $c^{s c i d} I l 2 \mathrm{rg}^{\mathrm{tm} I \mathrm{Wjl} / \mathrm{SzJ}}$ (NSG) neonatal mice. Based on our clonal analyses, I estimate that the transplanted populations contained between 375 and 850 hematopoietic progenitors, of which 62 to 140 cells were multipotent. CD $34^{+}$cord blood cells were transplanted as controls. Analyses of the bone marrow of recipients 4 weeks following transplantation showed engraftment only in animals that received CD $34^{+}$cord blood cells (Figure 3.21). These findings indicate that the $\mathrm{CD} 34^{+} \mathrm{CD} 45^{+} \mathrm{CD} 90^{+} \mathrm{CD} 7^{-}$population (at the cell numbers injected) does not contain progenitors capable of engrafting newborn recipients.

\subsubsection{Aldehyde dehydrogenase activity during hematopoietic development}

Studies of mouse hematopoietic development have demonstrated that RA signaling mediated by Raldh2 is required for the specification of HSCs and that AGM-derived HSCs can be isolated based on this activity using the Aldefluor assay (Chanda et al., 2013). Studies in the human have also shown that cord blood HSCs can be isolated based on Aldefluor activity (Fallon et al., 2003; Hess et al., 2004). To determine whether RA signaling could play a role in the specification and development of the human yolk sac programs, I measured ALDHs with the Aldefluor assay in $\mathrm{CD} 34^{+} \mathrm{CD} 45^{+} \mathrm{CD} 90^{+} \mathrm{CD} 7^{-}$cells, as well as in mesoderm (days 3 and 4 of differentiation) and HECcontaining populations (days 5 and 6 of differentiation) from which they were generated. As shown in Figures 3.22a to 3.22c, most of the populations did not contain $\mathrm{ALDH}^{+}$cells. While $\mathrm{ALDH}^{+}$ cells were detected on day 6 of differentiation, they were restricted to the CD34- fraction, which likely represents a non-hematopoietic population (Figure 3.22c). In contrast, populations specified to the definitive fate contained readily detectable $\mathrm{ALDH}^{+}$populations across days 3 to 6 of differentiation (Figure 3.22b and 3.22c). I also analyzed the expression of $A L D H 1 A 2$, which encodes RALDH2, between days 0 and 6 of differentiation. I found that $A L D H 1 A 2$ is consistently expressed at low levels across these stages of culture (Figure 3.22d), presumably too low to generate sufficient enzymatic activity to be detected by the Aldefluor assay. In contrast to the pattern of $A L D H 1 A 2$, expression of CYP26A1, the enzyme responsible for the degradation of RA, 

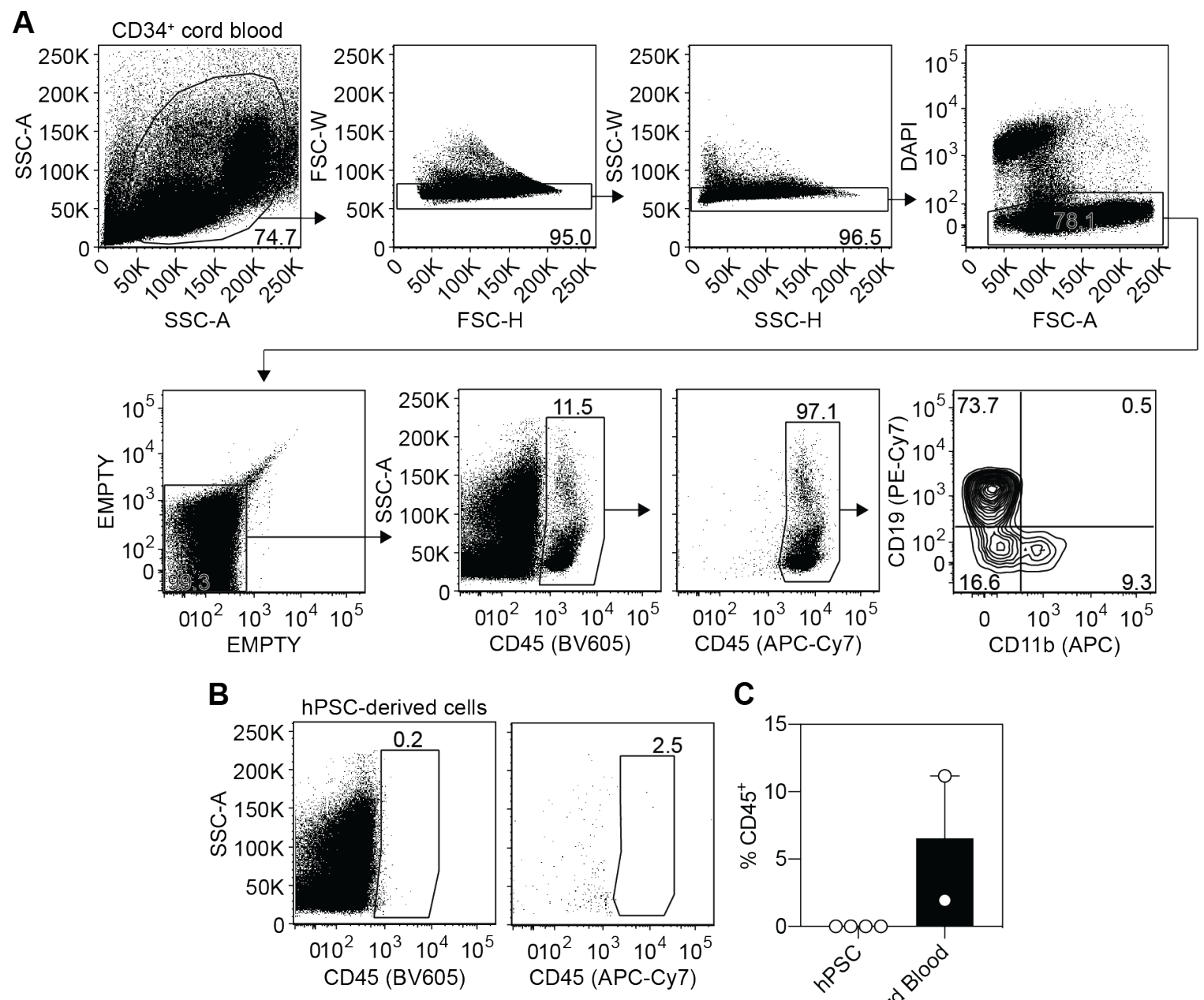

C

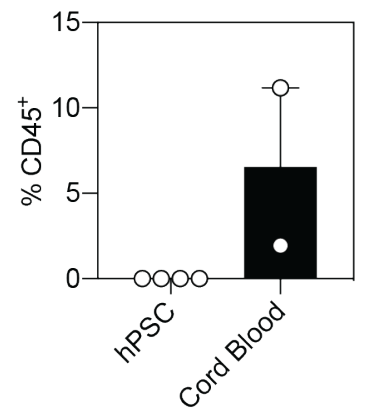

Figure 3.21 Engraftment potential of hPSC-derived multipotent hematopoietic progenitors.

A. Representative flow cytometric analysis of human CD45, CD19 and CD11b expression on cells in the bone marrow of recipients 4 weeks following transplantation of $\mathrm{CD} 34^{+}$cord blood cells.

B. Representative flow cytometric analysis of human CD45 expression on cells in the bone marrow of recipients 4 weeks following transplantation of hPSC-derived $\mathrm{CD} 34^{+} \mathrm{CD} 45^{+} \mathrm{CD} 90^{+} \mathrm{CD}^{-}$multipotent progenitor cells.

C. Quantification of the proportion of human $\mathrm{CD}^{2} 5^{+}$cells in the mouse bone marrow 4 weeks after transplantation $(n=2-4)$. 

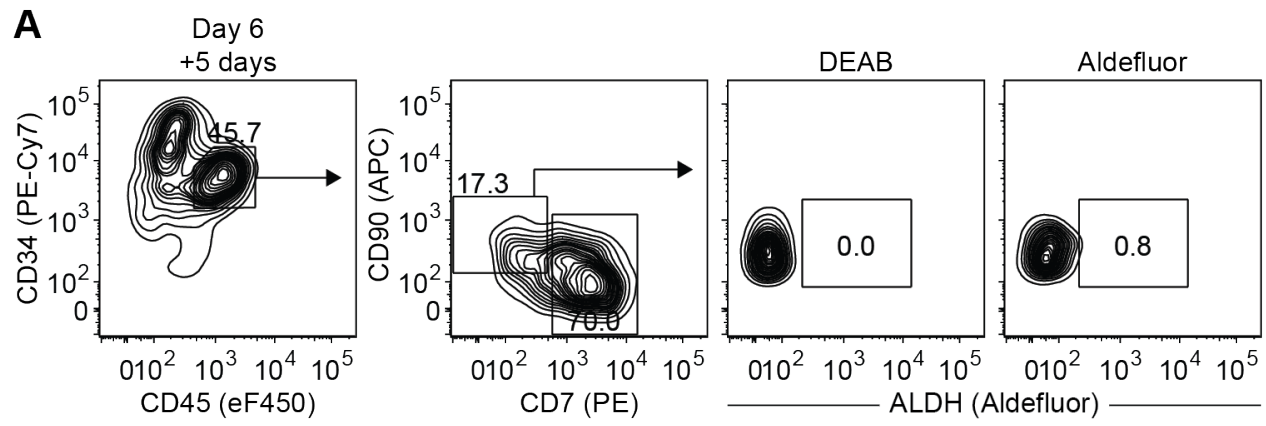

B

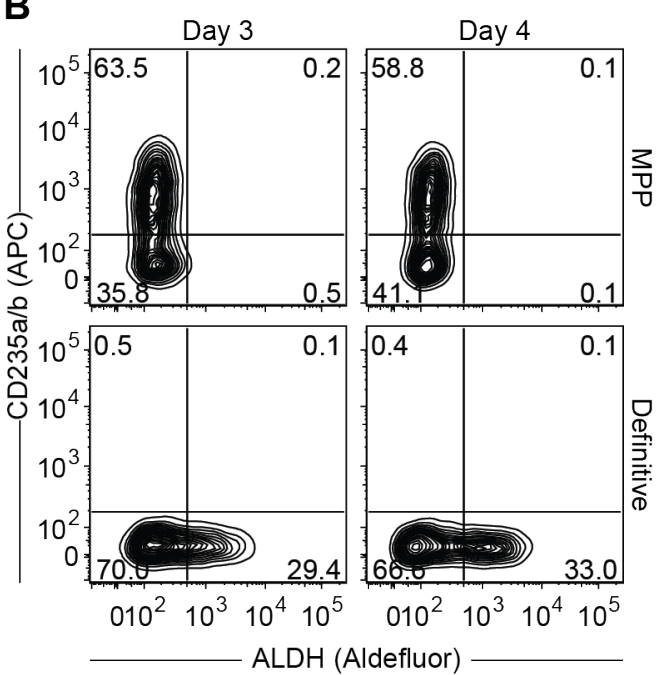

C
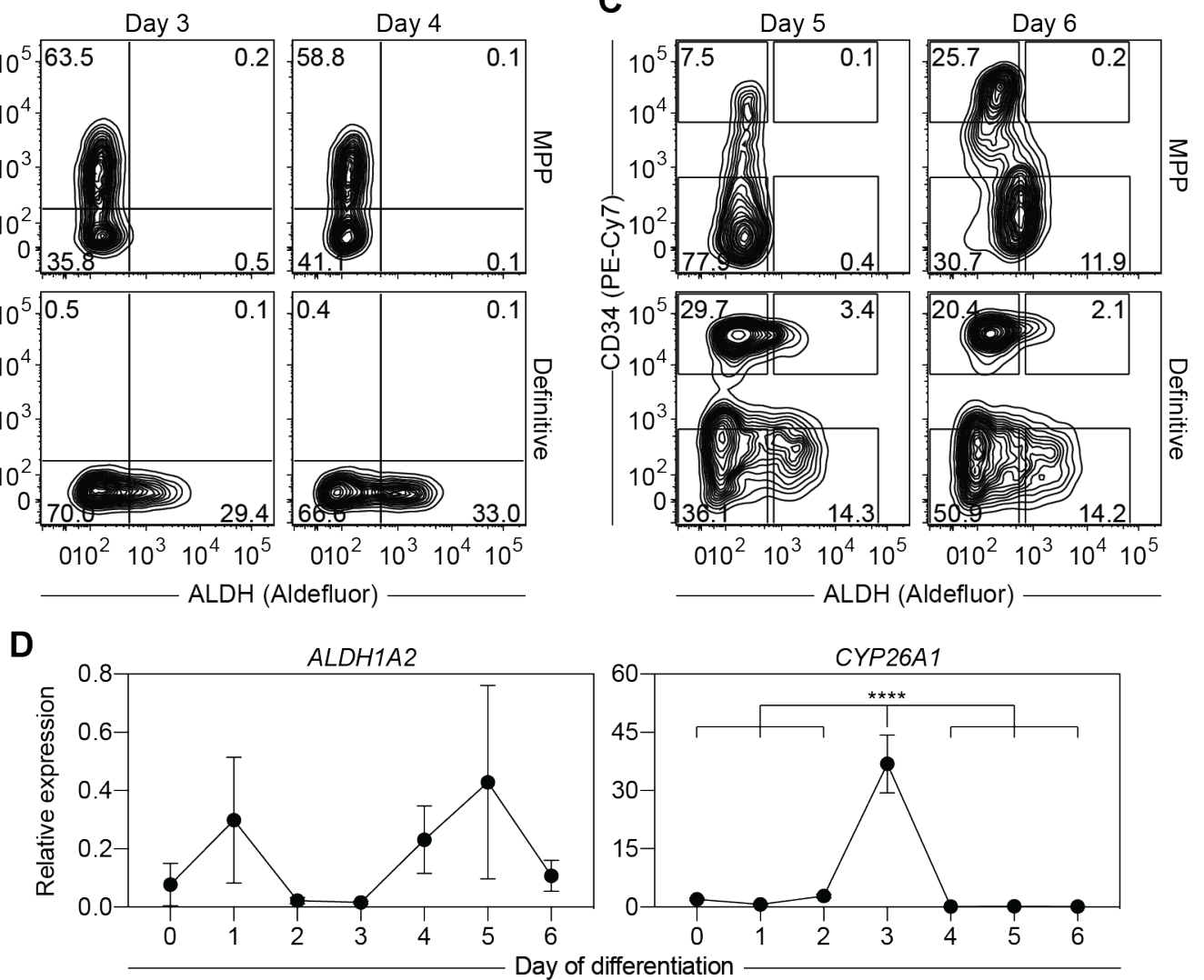

Figure 3.22 The primitive and multipotent progenitor programs do not develop from $\mathrm{ALDH}^{+}$progenitors.

A. Representative flow cytometric analysis of ALDH activity in the $\mathrm{CD} 34^{+} \mathrm{CD} 45^{+} \mathrm{CD} 90^{+} \mathrm{CD} 7^{-}$ population generated from day $6 \mathrm{CD} 34^{+} \mathrm{CD} 43^{-}$cells following 5 days of culture with OP9-DL4 cells.

B. Representative flow cytometric analysis of CD235a/b expression and ALDH activity in the primitive/MPP and definitive populations on days 3 and 4 of differentiation.

C. Representative flow cytometric analysis of CD34 expression and ALDH activity in the primitive/MPP and definitive populations on days 5 and 6 of differentiation.

D. RT-qPCR analysis of ALDH1A2 and CYP26A1 expression between days 0 and 6 of differentiation in primitive/MPP-induced populations $(\mathrm{n}=5)$. ANOVA. ${ }^{* * * *} P<0.0001$ versus the indicated sample. 
showed a sharp peak at day 3 of differentiation (Figure 3.22d). Taken together, these findings suggest that RA signaling does not play a role in the generation of the human primitive or MPP programs. Rather, the high levels of expression of CYP26A1 at day 3 of differentiation suggests that $\mathrm{CD} 235 \mathrm{a} / \mathrm{b}^{+}$mesoderm is safeguarded from exogenous RA signaling.

\subsubsection{Hematopoietic development from human pluripotent stem cell lines}

To determine whether the protocol for the generation of the human yolk sac hematopoietic programs is broadly applicable to other hPSC lines, I then tested an iPSC line (CHOP10WT) generated from the peripheral blood of a healthy donor (Maguire et al., 2016). Realizing that hPSC lines differ in their responsiveness to cytokines, I titrated Activin A ( 0 to $10 \mathrm{ng} / \mathrm{mL})$ in the presence of BMP4 $(10 \mathrm{ng} / \mathrm{mL})$ and FGF2 $(5 \mathrm{ng} / \mathrm{mL})$ between days 1 and 4 of differentiation to optimize the induction of $\mathrm{KDR}^{+} \mathrm{CD} 235 \mathrm{a} / \mathrm{b}^{+}$mesoderm. Populations induced with either 2 or $4 \mathrm{ng} / \mathrm{mL}$ of Activin A generated the highest proportion of $\mathrm{KDR}^{+} \mathrm{CD} 235 \mathrm{a} / \mathrm{b}^{+}$cells at day 4 of differentiation (Figure $3.23 \mathrm{a}$ and 3.23b). I further differentiated the mesoderm induced with $2 \mathrm{ng} / \mathrm{mL}$ of Activin $\mathrm{A}$ in the presence of VEGF, FGF2 and hematopoietic cytokines to evaluate the hematopoietic potential of the population. $\mathrm{CD} 43^{+}$hematopoietic cells appeared on day 6 of differentiation and increased in number over an additional 6 days of differentiation (Figure 3.23c and 3.23d) consistent with the pattern observed in previous studies using $\mathrm{H} 1 \mathrm{hESC}$ (Figure $3.2 \mathrm{~b}$ and $3.2 \mathrm{c}$ ). These findings indicate that the induction of $\mathrm{KDR}^{+} \mathrm{CD} 235 \mathrm{a} / \mathrm{b}^{+}$mesoderm in different $\mathrm{hPSC}$ lines require different concentrations of Activin A and highlights the importance of optimizing this stage of the protocol for each line.

I next asked whether the $\mathrm{KDR}^{+} \mathrm{CD} 235 \mathrm{a} / \mathrm{b}^{+}$mesoderm generated from the $\mathrm{CHOP} 10 \mathrm{WT}$ iPSCs gives rise to the primitive hematopoietic program and $\mathrm{T}$ lymphoid lineages. To investigate this, $\mathrm{I}$ isolated $\mathrm{KDR}^{+} \mathrm{CD} 235 \mathrm{a} / \mathrm{b}^{+}$mesoderm by FACS and cultured the cells as aggregates in the presence of VEGF, FGF2 and hematopoietic cytokines (Figure 3.24a). A CD $34^{+} \mathrm{CD}^{-} 3^{-}$population appeared after 1 day of culture and preceded the emergence of $\mathrm{CD}_{4} 3^{+}$hematopoietic cells, which were generated over the subsequent stages of the culture (Figure 3.24b and 3.24c). Analyses of colonyforming potential after 5 days of culture confirmed that $\mathrm{KDR}^{+} \mathrm{CD} 235 \mathrm{a} / \mathrm{b}^{+}$mesoderm gives rise to the primitive hematopoietic program (Figure 3.24d). I also observed T lymphoid progenitors following the culture of $\mathrm{KDR}^{+} \mathrm{CD} 235 \mathrm{a} / \mathrm{b}^{+}$-derived cells with OP9-DL4 cells for 1 month (Figure 3.24e). Taken together, these data demonstrate that it is possible to generate $\mathrm{KDR}^{+} \mathrm{CD} 235 \mathrm{a} / \mathrm{b}^{+}$ 
mesoderm with primitive erythroid and T lymphoid potential from different hPSC lines using the BMP4, FGF2 and Activin A-based protocol described in this thesis. 
A

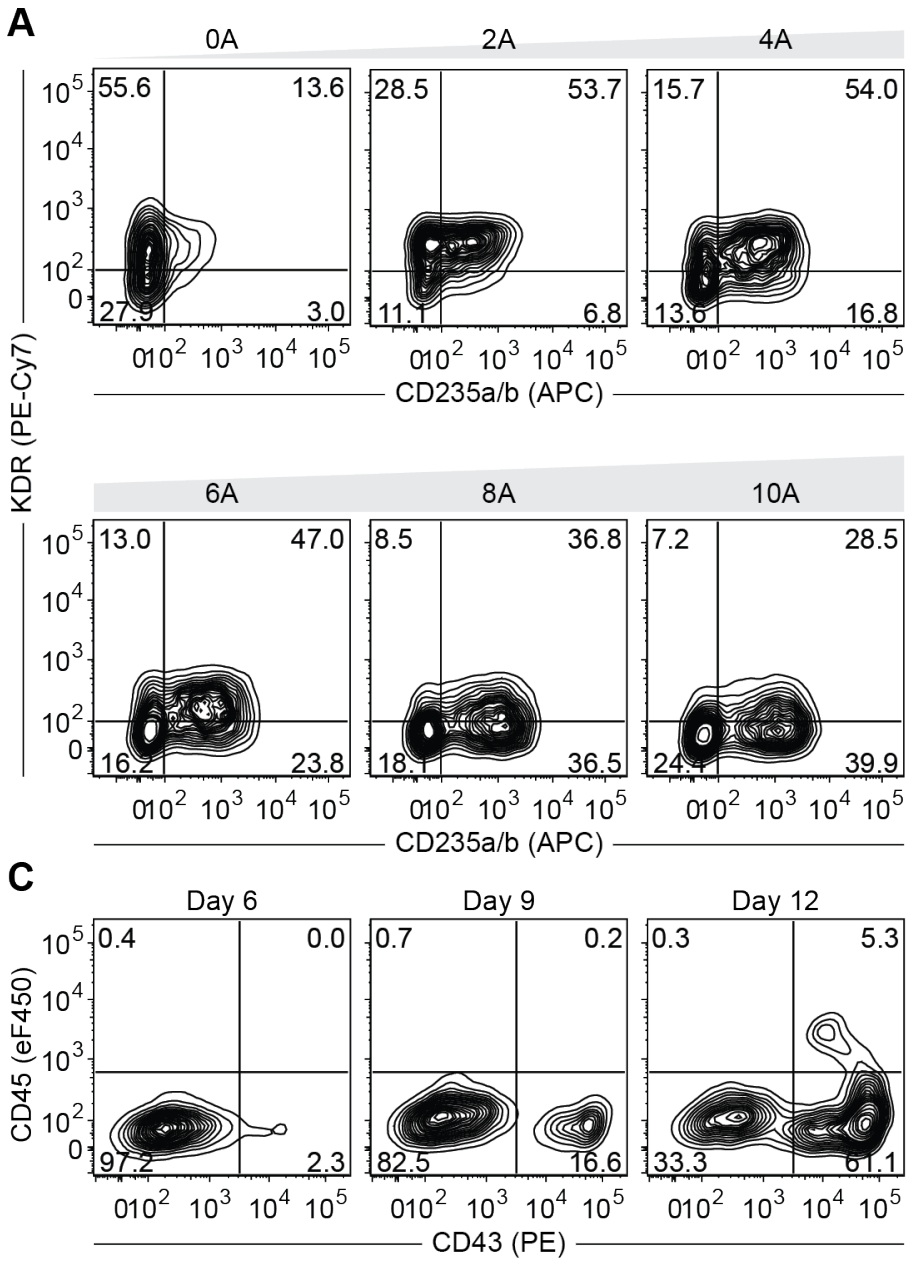

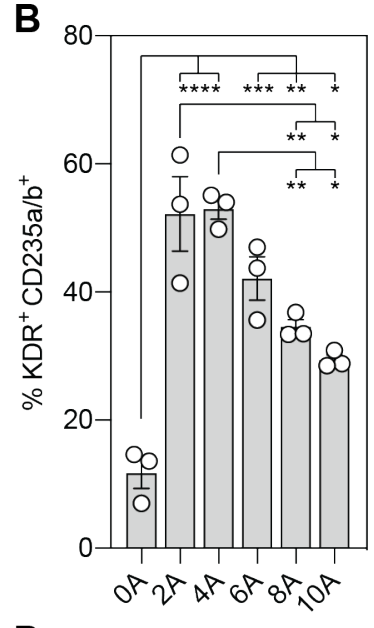

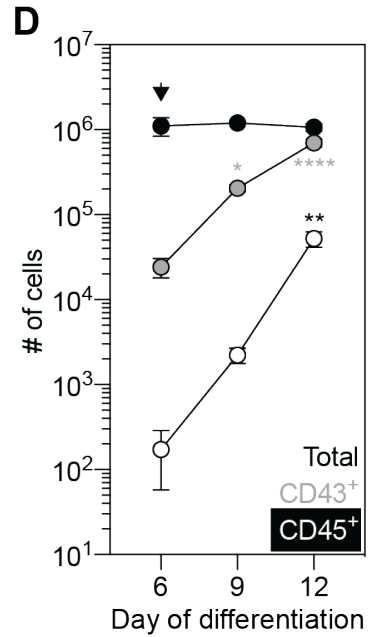

Figure 3.23 Generation of the yolk sac hematopoietic lineage from CHOP10WT iPSCs.

A. Representative flow cytometric analysis of KDR and CD235a/b expression on day 4 of differentiation.

B. Quantification of the proportion of $\mathrm{KDR}^{+} \mathrm{CD} 235 \mathrm{a} / \mathrm{b}^{+}$cells on day 4 of differentiation $(\mathrm{n}=3)$. ANOVA. ${ }^{*} P<0.05, * * P<0.01, * * * P<0.001$ and $* * * * P<0.0001$ versus the indicated sample.

C. Representative flow cytometric analysis of CD43 and CD45 expression on populations between days 6 and 12 of differentiation.

D. Quantification of the number of total and $\mathrm{CD} 43^{+}$and $\mathrm{CD} 45^{+}$all cells in the populations between days 6 and 12 of differentiation $(\mathrm{n}=3)$. ANOVA. $* P<0.05, * * P<0.01$ and $* * * * P$ $<0.0001$ versus the indicated population on day 6 of differentiation. 

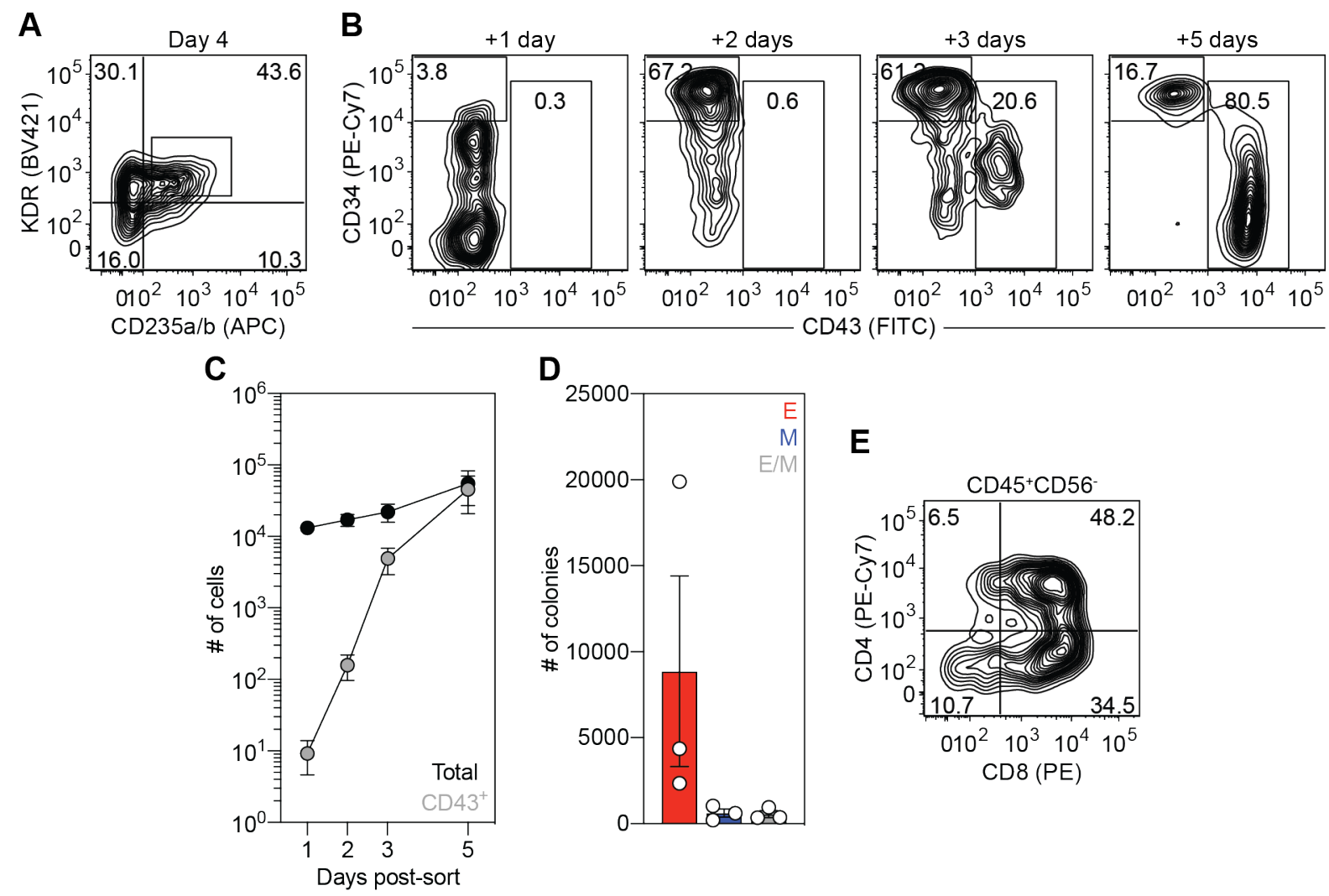

$\mathbf{E}$

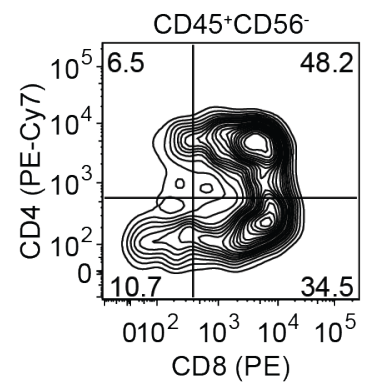

Figure 3.24 Hematopoietic potential of $\mathrm{KDR}^{+} \mathrm{CD} 235 \mathrm{a} / \mathrm{b}^{+}$mesoderm generated from CHOP10WT iPSCs.

A. Gating strategy used for FACS-based isolation of the $\mathrm{KDR}^{+} \mathrm{CD} 235 \mathrm{a} / \mathrm{b}^{+}$population on day 4 of differentiation.

B. Representative flow cytometric analysis of CD34 and CD43 expression on aggregate populations generated from the day $4 \mathrm{KDR}^{+} \mathrm{CD} 235 \mathrm{a} / \mathrm{b}^{+}$cells.

C. Quantification of the number of total and $\mathrm{CD} 43^{+}$cells generated from 62,500 day 4 $\mathrm{KDR}^{+} \mathrm{CD} 235 \mathrm{a} / \mathrm{b}^{+}$cells $(\mathrm{n}=3)$. ANOVA. not significant.

D. Colony-forming progenitor number generated from 62,500 day $4 \mathrm{KDR}^{+} \mathrm{CD} 235 \mathrm{a} / \mathrm{b}^{+}$cells following 5 days of culture $(\mathrm{n}=3)$. ANOVA. not significant. Colonies: $\mathrm{E}=$ erythroid (red), $\mathrm{M}$ $=$ myeloid (blue) and $\mathrm{E} / \mathrm{M}=$ mixed erythro-myeloid (grey).

E. Representative flow cytometric analysis of CD4 and CD8 expression on the CD45 ${ }^{+}$CD56 population generated from day $4 \mathrm{KDR}^{+} \mathrm{CD} 235 \mathrm{a} / \mathrm{b}^{+}$cells following 30 days of culture with OP9-DL4 cells. 


\subsection{Summary}

In this chapter, I describe the development of the human yolk sac hematopoietic programs (Figure 3.25). These lineages are generated from $\mathrm{KDR}^{+} \mathrm{CD} 235 \mathrm{a} / \mathrm{b}^{+}$mesoderm that was induced with specific concentrations of BMP4, FGF2 and Activin A. Within 24 hours, this mesoderm generates primitive HECs that quickly undergo NOTCH-dependent EHT to produce hematopoietic progenitors. These cells give rise to primitive erythroid, mast cell and MYB-independent macrophage progeny. As primitive hematopoietic progenitors emerge, I show that a second population of HECs is present. These HECs undergo EHT to give rise to multipotent hematopoietic progenitors that can be distinguished from primitive progenitors based on the expression of CD45. These MPPs show EMP erythroid, mast cell, macrophage, granulocyte, NK cell and T lymphoid potential consolidating the EMP and LMP programs. This work puts forward a new model of yolk sac hematopoietic development and the implications of these findings will be discussed in Chapter 5. 


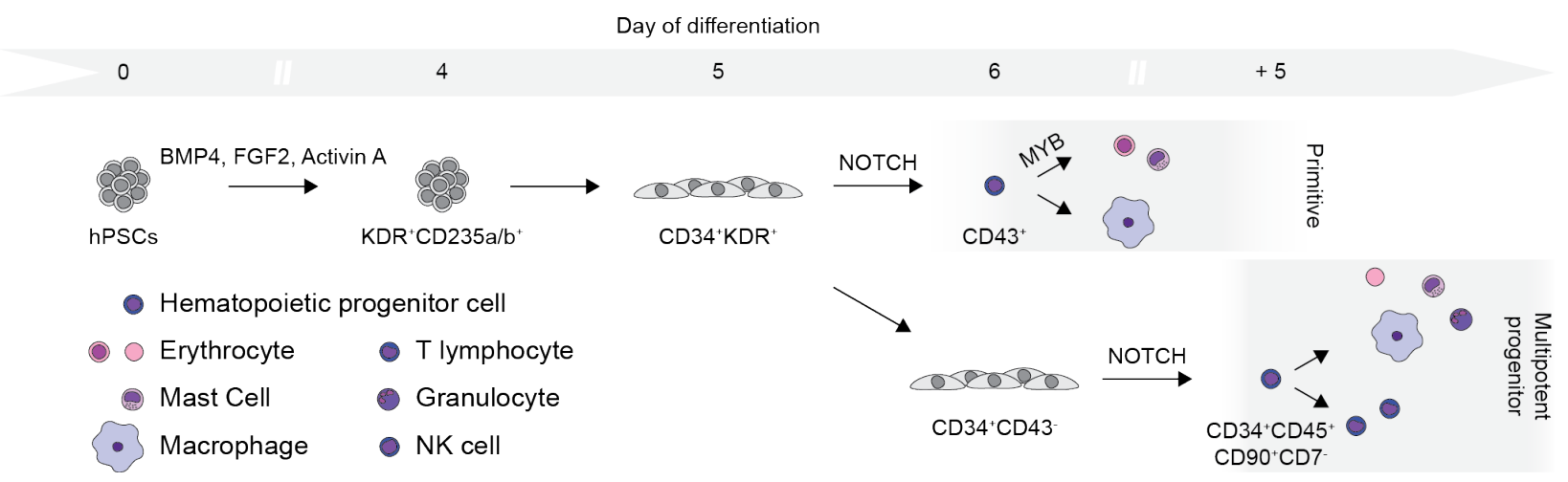

Figure 3.25 Model of human yolk sac hematopoietic development using pluripotent stem cells.

In this study, I show that the concurrent addition of BMP4, FGF2 and Activin A promote the generation of hPSC differentiation cultures that are highly enriched in $\mathrm{KDR}^{+} \mathrm{CD} 235 \mathrm{a} / \mathrm{b}^{+}$mesoderm at day 4 of differentiation. Within 24 hours, the mesoderm gives rise to HECs on day 5 of differentiation that undergo NOTCH-dependent EHT to generate the hematopoietic progenitors of the primitive hematopoietic program. We show that the primitive hematopoietic program gives rise to primitive erythroid, mast cells and macrophage lineages. With the exception of the macrophage lineage, the differentiation of the primitive hematopoietic progenitors requires MYB. Coincident with the emergence of the hematopoietic progenitors of the primitive hematopoietic program on day 6 of differentiation, we show that a second population of HECs give rise to multipotent hematopoietic progenitors that can be distinguished from their primitive counterpart based on the expression of CD34, CD45, CD90 and CD7. The multipotent progenitor program represents the unification of the EMP and LMP programs, and gives rise to the erythroid, mast cell, macrophage, NK cell and T lymphoid lineages. 


\section{Chapter 4}

\section{Clonal analysis of hematopoietic mesoderm}

This chapter contains ongoing studies.

\subsection{Acknowledgements}

I thank the SickKids/UHN Flow Cytometry Facility for their assistance with cell sorting.

\subsection{Primer}

Clonal analyses of the early stages of mouse hematopoietic development have shown that single BL-CFCs (hemangioblasts) give rise to both the primitive and EMP programs, suggesting that the different yolk sac hematopoietic programs are specified from a common mesoderm. Our studies in the hPSC differentiation system have shown that the EMP program has broader lineage potential than what was originally described in the mouse and displays the ability to generate the $\mathrm{T}$ lymphoid, as well as the erythroid and myeloid lineages. As the primitive and MPP programs are both derived from $\mathrm{KDR}^{+} \mathrm{CD} 235 \mathrm{a} / \mathrm{b}^{+}$mesoderm, I was next interested in determining if the yolk sac programs also develop from a common mesoderm. These studies are important, as they will provide new insights into the specification of human hematopoietic cell fates from mesoderm. Specifically, they address the question of whether or not the primitive erythroid and T lymphoid lineages develop from the same progenitor. Such an observation would demonstrate that the yolk sac hematopoietic programs are specified simultaneously through a common mesoderm with primitive and MPP potential. To address this, I am following two different approaches. The first method is to analyze human BL-CFC-derived blast colonies for the presence of primitive erythroid and $\mathrm{T}$ lymphoid potential. I focused on these lineages as they are unique to the primitive and MPP programs, respectively. The second method is to track the primitive erythroid and $\mathrm{T}$ lymphoid potential of single $\mathrm{KDR}^{+} \mathrm{CD} 235 \mathrm{a} / \mathrm{b}^{+}$cells by lentiviral integration site analysis.

\subsection{Results}

\subsubsection{Development and potential of BL-CFC-derived blast colonies}

To track the development of BL-CLC-derived blast colonies, I performed serial imaging of methylcellulose cultures generated from cells from whole day 4 differentiated populations to 
determine the stages that primitive erythroid and MPP HECs might be contained within the culture. Small colonies appeared within 1 day of culture. Although tight-bordered colonies were present at early stages of colony development, this morphology gave way to a scalloped-bordered architecture at later stages of culture (Figure 4.1a). I next collected the contents of the culture on each day and performed flow cytometric analyses to determine when HECs and hematopoietic cells appear. A population of $\mathrm{KDR}^{+} \mathrm{CD} 34^{+}$cells appeared within 1 day and persisted over the 4 day culture period (Figure 4.1b). $\mathrm{CD}^{+} 3^{+}$hematopoietic cells emerged 24 hours later (2 days of culture) and became the dominant population by 4 days of culture (Figure $4.1 \mathrm{c}$ ). These data indicate that HEC and hematopoietic progenitors are generated within BL-CFC cultures at similar stages to those defined in experiments performed in liquid cultures (described in Chapter 3). This timeline provides a platform to study hematopoietic commitment from the BL-CFC.

Studies of mESC and hPSC differentiation have demonstrated that TGF- $\beta 1$ inhibits the generation of BL-CFC-derived blast colonies and impaired hematopoiesis (Bai et al., 2013; Park et al., 2004; Perlingeiro, 2007; Wang et al., 2012). Given the inclusion of serum, which contains TGF- $\beta 1$ in the methylcellulose cultures, I questioned whether the serum TGF- $\beta 1$ impairs the development of human blast colonies. To investigate this possibility and optimize hematopoietic development in methylcellulose, I analyzed the effect of inhibiting this pathway on colony growth. For these studies, cells from day 4 of differentiation were cultured the presence or absence of the small molecule TGF- $\beta$ R inhibitor, SB431542. As shown in Figure 4.2a and 4.2b, the addition of the inhibitor led to an increase in both the number and size of developing blast colonies. Flow cytometric analyses also showed that TGF- $\beta$ inhibition increased the proportion of CD43 ${ }^{+}$ hematopoietic cells within the colonies at all stages analyzed (Figure 4.2c). Together, these data show that TGF- $\beta$ signaling impairs early human hematopoietic development from the BL-CFC and that it is possible to improve the sensitivity of the hemangioblast assay by inhibiting this pathway. 

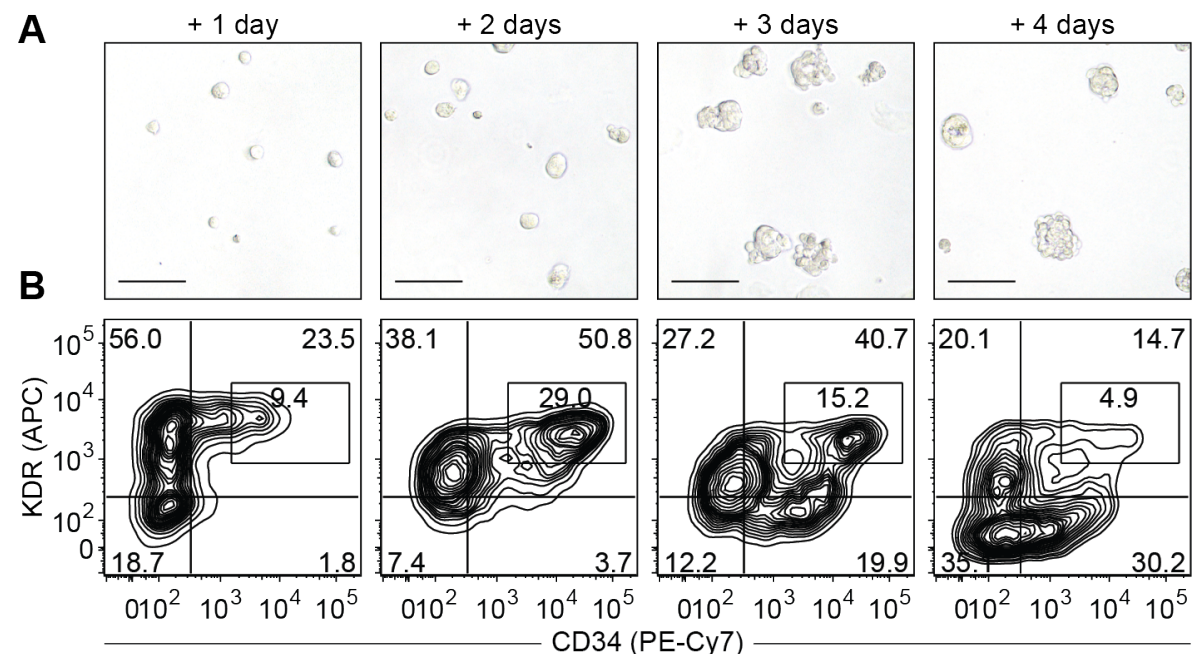

C
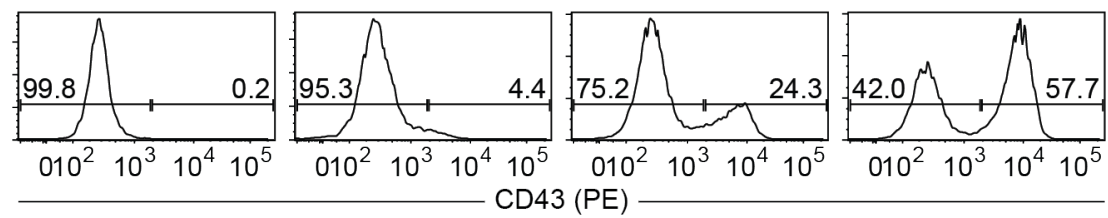

Figure 4.1 BL-CFC-derived blast colony development.

A. Representative brightfield images of developing blast colonies over 4 days of BL-CFC culture. Scale bar $=50 \mu \mathrm{m}$.

B. Representative flow cytometric analysis of KDR and CD34 expression over 4 days of BL-CFC culture.

C. Representative flow cytometric analysis of CD43 expression over 4 days of BL-CFC culture. 

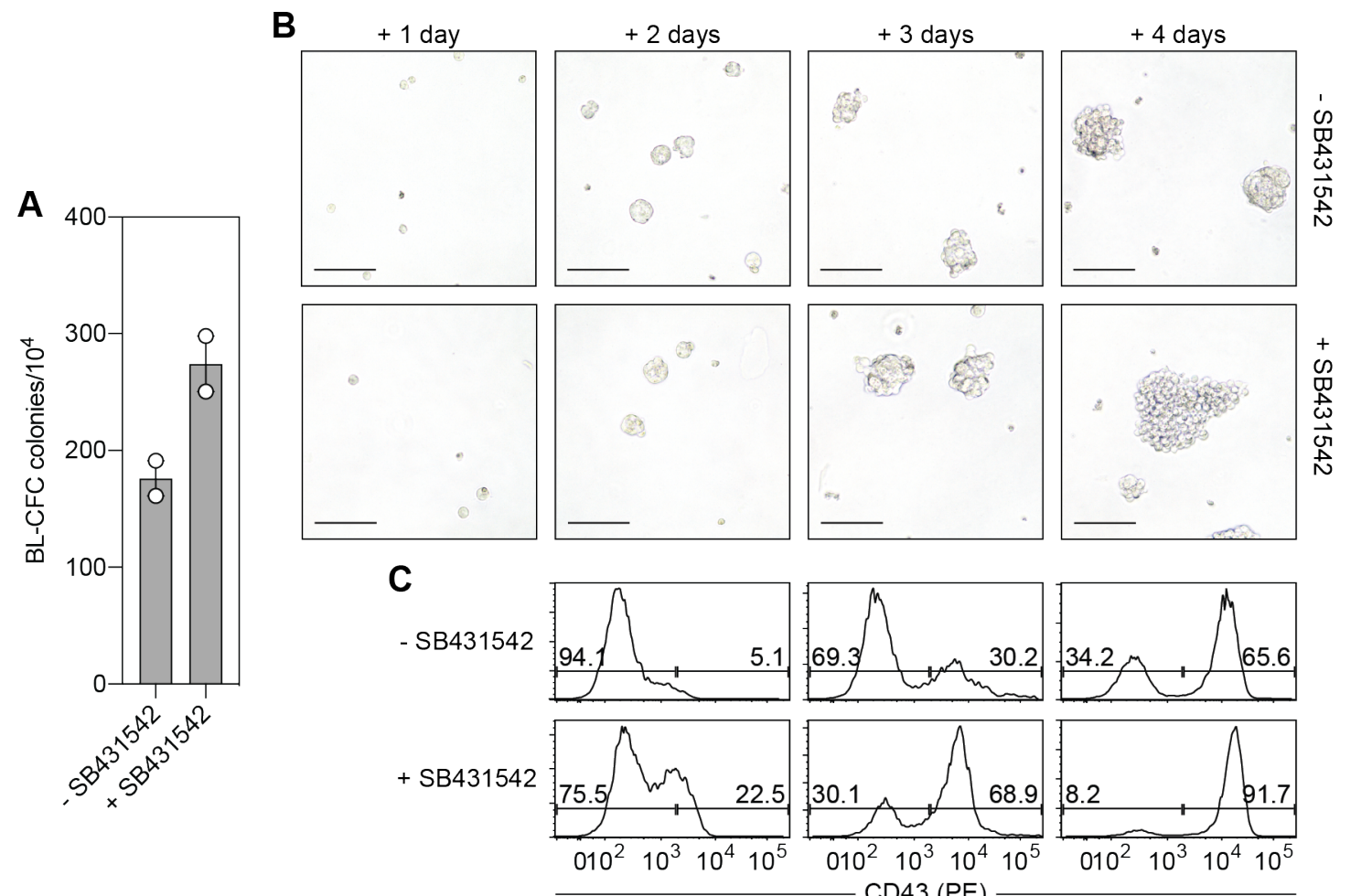

Figure 4.2 The addition of the TGF $\beta$ inhibitor, SB431542 enhances BL-CFC-derived blast colony development.

A. BL-CFC frequency of the day 4 populations cultured in the presence or absence of SB431542 $(\mathrm{n}=2)$. Colonies were scored after day 6 of culture.

B. Representative brightfield images of developing blast colonies grown in the presence or absence of SB431542. Scale bar $=50 \mu \mathrm{m}$.

C. Representative flow cytometric analysis of CD43 expression on cells in developing blast colonies generated isolated from methylcellulose cultures in the presence or absence of SB431542. 
I next asked whether the developing BL-CFC cultures supported the development of progenitors of the primitive and multipotent progenitor programs. As a first step, I pooled the contents of entire cultures and assayed the cells in methylcellulose to measure colony-forming progenitor potential and cultured the cells with OP9-DL4 cells to evaluate T lymphoid potential. The pooled cultures contained primitive erythroid and $\mathrm{T}$ lymphoid progenitors indicating that these fates are specified early in development (Figure 4.3a and 4.3b). T lymphoid progenitors were detected from entire cultures with or without SB431542 (Figure 4.3b). Collectively, these findings show that the conditions used to generate blast colonies promotes the development of the primitive and MPP programs from day 4 mesoderm-containing populations.

Given these findings, I next set out to demonstrate that the primitive erythroid and $\mathrm{T}$ lymphoid progenitors are generated within blast colonies. For these analyses, I first cultured pools of 10 or 30 blast colonies with OP9-DL4 cells to gauge the proportion of colonies that could give rise to $\mathrm{CD} 34^{+} \mathrm{CD} 45^{+}$progenitors under these conditions. While wells seeded with 10 colonies failed to generate $\mathrm{CD} 34^{+} \mathrm{CD} 45^{+}$cells after 4 days of culture with OP9-DL4 cells, $60 \%$ of the wells seeded with 30 colonies gave rise to $\mathrm{CD} 34^{+} \mathrm{CD} 45^{+}$cells (Figure 4.4). The lack of hematopoietic cell development from pools of 10 colonies indicates that either the colonies contain relatively few progenitors that can survive and expand on OP9-DL4 cells under these conditions or that a small population $(<10 \%)$ of the colonies contain progenitors with $\mathrm{T}$ lymphoid potential.

To overcome the challenges associated with analyses of single blast colonies, I next investigated the possibility of detecting $\mathrm{T}$ lymphoid potential from single $\mathrm{KDR}^{+} \mathrm{CD} 235 \mathrm{a} / \mathrm{b}^{+}$cells in liquid culture, as this population readily gives rise to T lymphocytes (Figure 3.16). This approach failed, as the viability of mesoderm cells is poor when cultured in isolation (data not shown). 

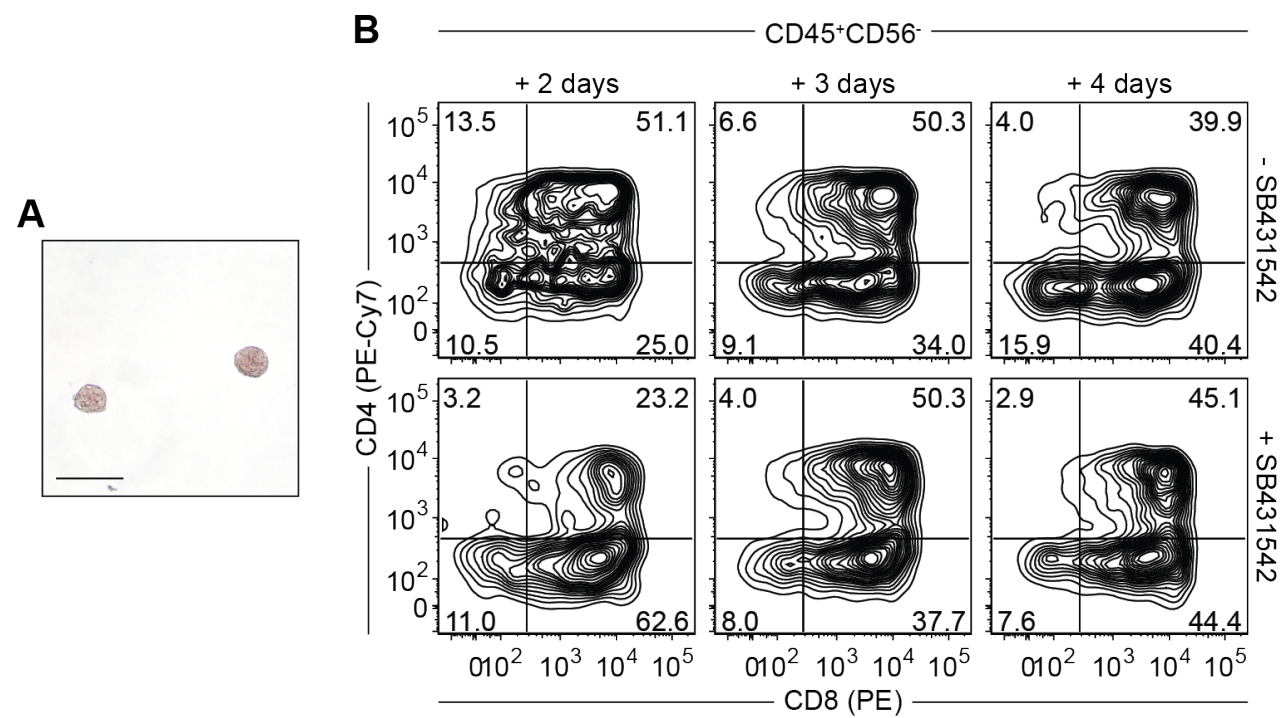

Figure 4.3 Hematopoietic potential of BL-CFC-derived cultures.

A. Representative brightfield image of primitive erythroid colonies generated from BL-CFC cultures. Scale bar $=50 \mu \mathrm{m}$.

B. Representative flow cytometric analysis of CD4 and CD8 expression on the CD45 ${ }^{+} \mathrm{CD}_{5}{ }^{-}$ population generated from content of entire BL-CFC cultures following 30 days of culture with OP9-DL4 cells. 

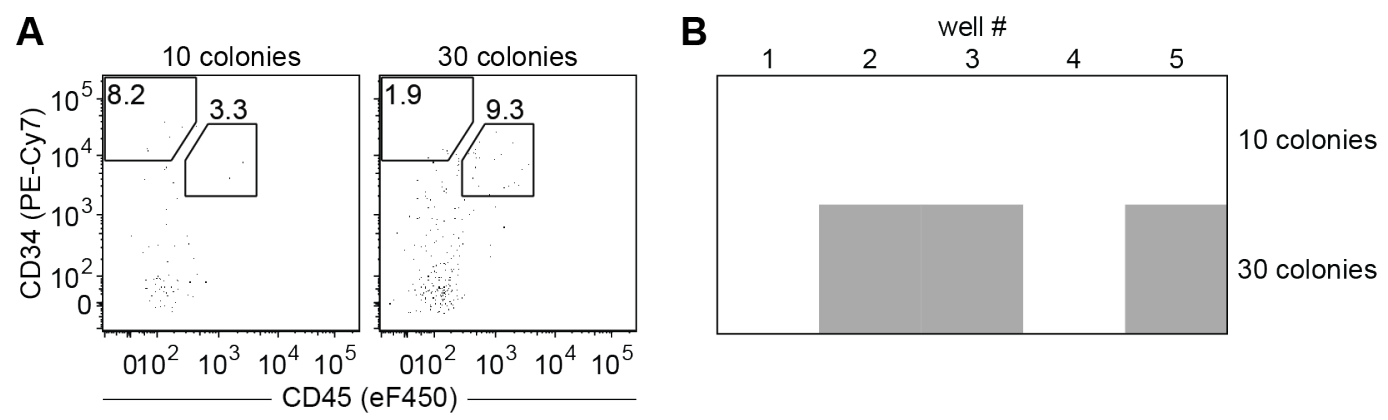

Figure 4.4 BL-CFC-derived blast colonies do not efficiently give rise to $\mathrm{CD} 34^{+} \mathrm{CD} 45^{+}$cells
A. Representative flow cytometric analysis of CD34 and CD45 expression on populations generated from pools of either 10 or 30 blast colonies following culture with OP9-DL4 cells for 4 days.
B. Heatmap representing wells that gave rise to greater than $10 \mathrm{CD} 34^{+} \mathrm{CD} 45^{+}$cells (grey). 


\subsubsection{Clonal analysis hematopoietic mesoderm}

As a second approach, I turned to lentivirus-based clonal analyses. With this approach, clones are labelled pseudo-randomly (Schröder et al., 2002) by the insertion site of the lentiviral genome, which serves as a heritable marker of the cell and its progeny. The marker can be followed by PCR and sequencing-based analyses of the derivative populations, whereby the appearance of common integration sites in different lineages is indicative of a clonal relationship (Figure 4.5).

To virally mark the cells, we developed a spinoculation protocol to transduce FACS-isolated $\mathrm{KDR}^{+} \mathrm{CD} 235 \mathrm{a} / \mathrm{b}^{+}$mesoderm at day 4 of differentiation. With this approach, exposure to the lentivirus was restricted to a narrow window of time to mitigate the labeling of unipotent progenitors in the event that the primitive and MPP programs diverge between days 4 and 5 of differentiation. In addition, I transduced the $\mathrm{KDR}^{+} \mathrm{CD} 235 \mathrm{a} / \mathrm{b}^{+}$cells at a MOI less than 2 to ensure that no more than $10 \%$ of cells expressed eGFP after 3 days of culture (Figure 4.6a and 4.6b). To determine whether the number of $\mathrm{eGFP}^{+}$cells in the culture is proportional to the number of transduced $\mathrm{KDR}^{+} \mathrm{CD} 235 \mathrm{a} / \mathrm{b}^{+}$cells, I transferred a fraction of the transduced cells to methylcellulose to enumerate the proportion of blast colonies that express eGFP (Figure 4.6c). In the majority of experiments, there was a strong correlation between the frequency of $\mathrm{eGFP}^{+}$cells in liquid culture and the frequency of $\mathrm{eGFP}^{+}$blast colonies (Figure 4.6d). Lentiviral transduction did not affect the developmental potential of the mesoderm, as the proportion of $\mathrm{CD} 34^{+} \mathrm{CD}^{-} 3^{-}$and $\mathrm{CD} 3^{+}$cells in the $\mathrm{eGFP}^{+}$population was equivalent to that of the $\mathrm{eGFP}^{-}$population (Figure 4.6e and 4.6f). I next isolated the transduced $\mathrm{CD}^{+} 3^{+}$and $\mathrm{CD} 34^{+} \mathrm{CD} 43^{-}$populations by FACS and assayed erythroid colony-forming progenitor and T lymphoid potential, respectively (Figure 4.7a). Additional culture of the $\mathrm{CD} 43^{+}$population for 2 days was used to further expand the clones prior to their transfer into methylcellulose and led to the generation of small colonies of erythroblasts characteristic of the primitive hematopoietic program (Figure 4.7b), while the culture of the $\mathrm{CD} 34^{+} \mathrm{CD}^{-} 3^{-}$population with OP9-DL4 cells gave rise to T lymphoid progenitors after 3-4 weeks (Figure 4.7c). Collectively, these data indicate that lentiviral integration site is a viable strategy to assay the primitive erythroid and $\mathrm{T}$ lymphoid potentials of single $\mathrm{KDR}^{+} \mathrm{CD} 235 \mathrm{a} / \mathrm{b}^{+}$cells. Current studies are focused on determining whether common integration sites are present in primitive erythroid colonies and $\mathrm{CD} 45^{+} \mathrm{CD} 56^{-} \mathrm{CD} 4^{+} \mathrm{CD} 8^{+} \mathrm{T}$ lymphoid progenitors using next generation DNA sequencing of viral integration site PCR libraries. 


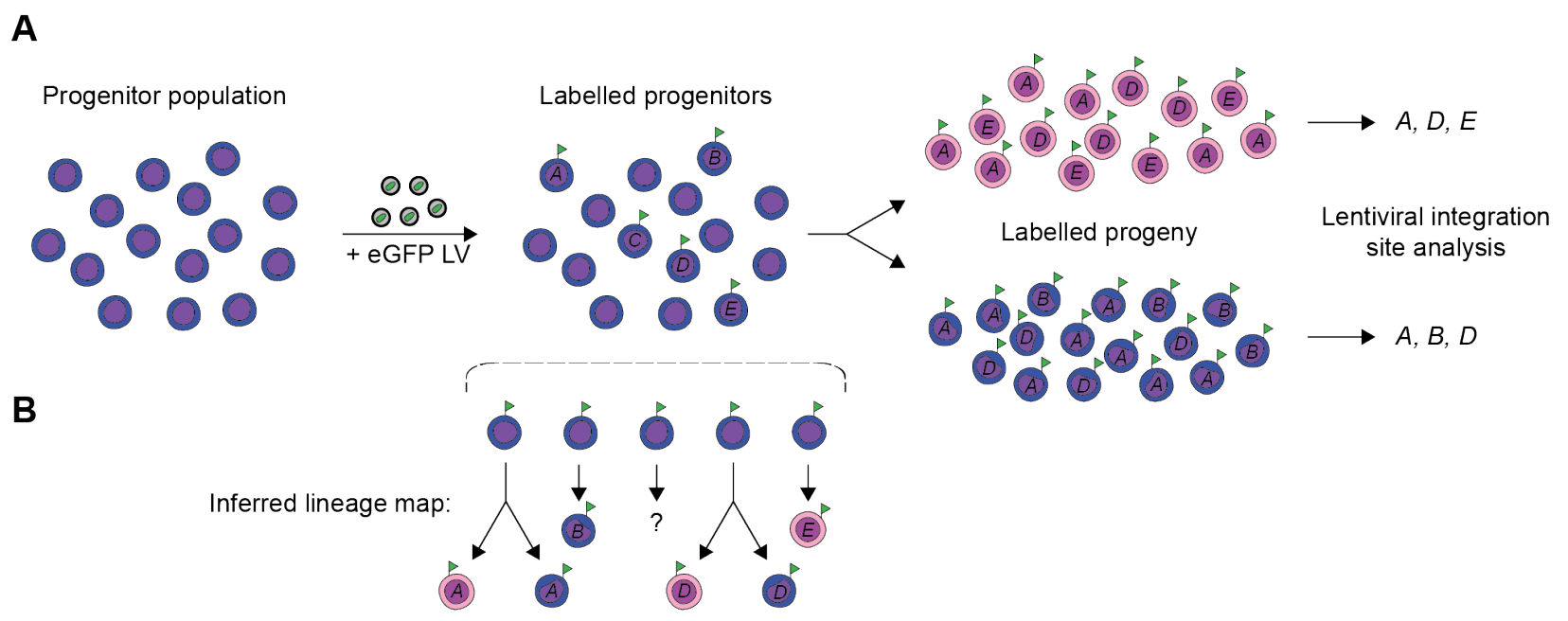

Figure 4.5 Lentivirus-based strategy to track the primitive erythroid and $\mathrm{T}$ lymphoid potential of single cells.

A. In this example, the progenitor population is transduced to label a fraction of the cells in the population. The transduced cells are uniquely marked by the insertion sites of the lentiviral genome, which are inherited by their progeny. DNA sequencing identifies the lentiviral integration sites in the differentiated populations. In this example, the primitive erythroid population contains integration site sequences $A, D$ and $E$, while the T lymphoid population contains the integration site sequences $A, B$ and $D$.

B. The presence of integration site sequences $A$ and $D$ in the two differentiated populations indicate that the two lineages emerged from a common progenitor. The appearance of integration site sequences $B$ and $E$ in only one of the two differentiated populations suggests that the clones developed from unilineage progenitors. Other cells that were labeled (eg. with integration site $C$ ) may be lost from the final analysis, as the progenitor fails to give rise to either of the two differentiated populations. 


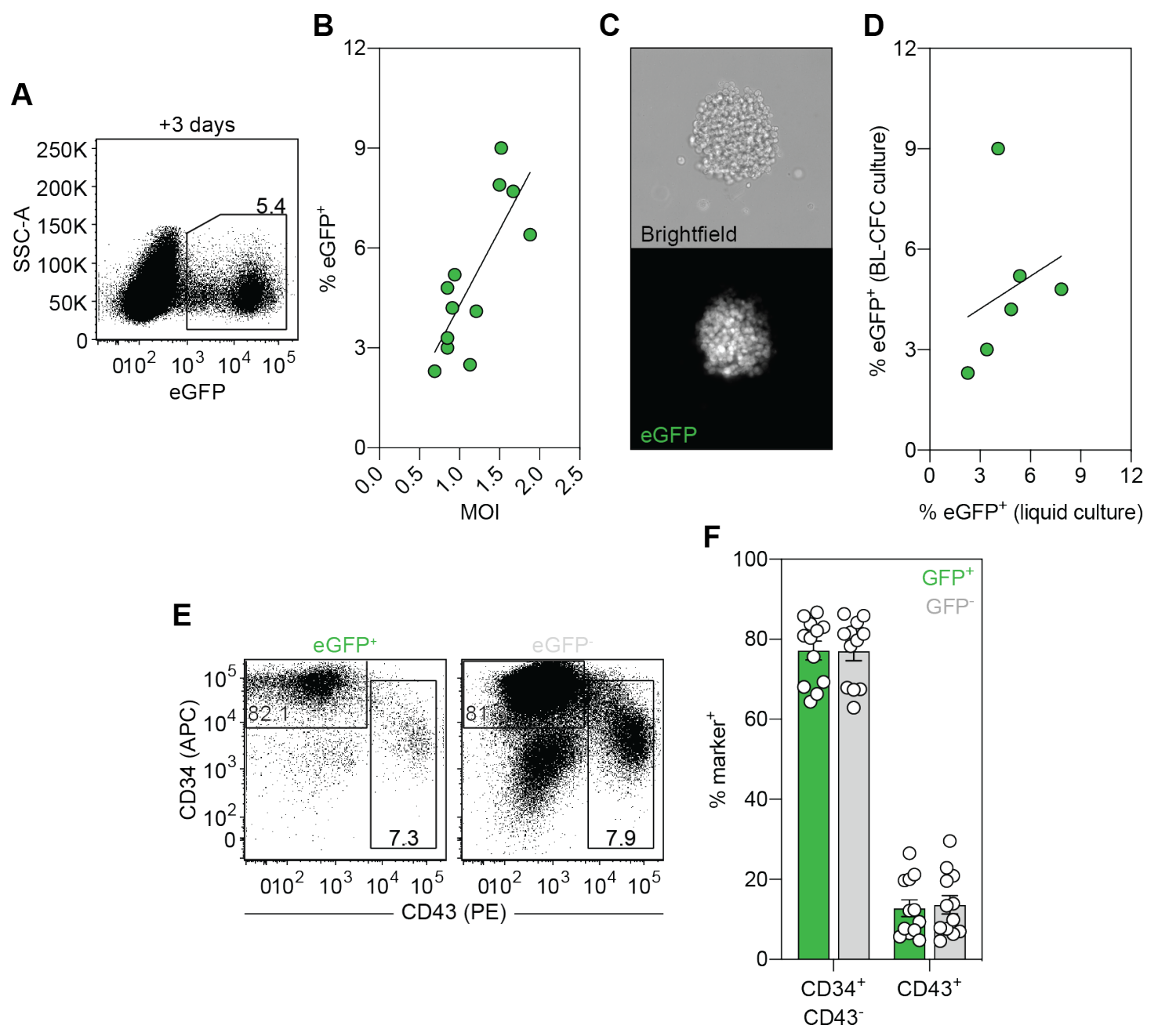

Figure 4.6 Lentiviral transduction of $\mathrm{KDR}^{+} \mathrm{CD} 235 \mathrm{a} / \mathrm{b}^{+}$mesoderm.

A. Representative flow cytometric analysis of eGFP expression in the transduced day 4 $\mathrm{KDR}^{+} \mathrm{CD} 235 \mathrm{a} / \mathrm{b}^{+}$mesoderm following 3 days of culture as aggregates.

B. Relationship between the proportion of $\mathrm{eGFP}^{+}$cells in A. and the multiplicity of infection (n $=12$ ). Linear regression. $R^{2}=0.61$.

C. Representative brightfield and fluorescence images of a blast colony generated from transduced $\mathrm{KDR}^{+} \mathrm{CD} 235 \mathrm{a} / \mathrm{b}^{+}$mesoderm.

D. Relationship between the proportion of $\mathrm{eGFP}^{+}$cells following 3 days in liquid culture and the proportion of $\mathrm{eGFP}^{+}$blast colonies following 6 days of methylcellulose culture $(\mathrm{n}=6)$. Linear regression. $R^{2}=0.07$.

E. Representative flow cytometric analysis of CD34 and CD43 expression on the transduced day $4 \mathrm{KDR}^{+} \mathrm{CD} 235 \mathrm{a} / \mathrm{b}^{+}$mesoderm-derived $\mathrm{eGFP}^{+}$and $\mathrm{eGFP}^{-}$populations following 3 days of culture as aggregates.

F. Quantification of proportion of the populations shown in E. $(n=12)$. ANOVA. Not significant. 

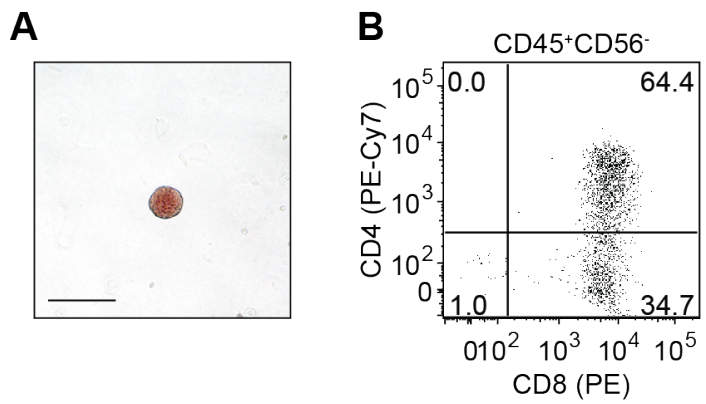

Figure 4.7 Lineage potential of $\mathrm{eGFP}^{+}$cells.

A. Representative brightfield image of a primitive erythroid colony generated from the isolated $\mathrm{eGFP}^{+} \mathrm{CD} 43^{+}$cells. Cells we cultured as aggregates for 2 additional days prior to their transfer to methylcellulose. Scale bar $=50 \mu \mathrm{m}$.

B. Representative expression of CD4 and CD8 on the CD45 ${ }^{+} \mathrm{CD} 56^{-}$population generated from $\mathrm{eGFP}^{+} \mathrm{CD} 34^{+} \mathrm{CD}^{-} 3^{-}$cells cultured with OP9-DL4 cells for 25 days. 


\section{Chapter 5}

\section{Summary}

\subsection{Major conclusions}

In this thesis, I have used hPSC differentiation to model the development of the blood cell lineages that I propose represent the hematopoietic programs initiated in the human yolk sac. The characterization of the developmental potential of these lineages and their relationship to one another is an essential step towards understanding how the human hematopoietic system develops. Additionally, this model provides access to the embryonic hematopoietic progenitor populations of tissue-resident immune cells that are otherwise inaccessible. To accomplish this goal, I used an approach that was guided by studies of model organism hematopoietic development and built on our previous work in the hPSC differentiation system that demonstrated that the expression of $\mathrm{CD} 235 \mathrm{a} / \mathrm{b}$ identifies the subset of $\mathrm{KDR}^{+}$mesoderm that gives rise to primitive, but not definitive hematopoiesis (Sturgeon et al., 2014). The hypothesis that I tested in my $\mathrm{PhD}$ project was that the $\mathrm{KDR}^{+} \mathrm{CD} 235 \mathrm{a} / \mathrm{b}^{+}$population represents the mesoderm that gives rise to the complete spectrum of human yolk sac hematopoiesis, including the primitive, EMP and LMP programs. The protocol that I designed to induce $\mathrm{KDR}^{+} \mathrm{CD} 235 \mathrm{a} / \mathrm{b}^{+}$mesoderm was modified from one optimized in our lab to generate ventricular cardiomyocytes (Lee et al., 2017) and efficiently supported the development of the different hematopoietic programs. With this approach, I was able to make the following new observations. First, I showed that the concurrent addition of the mesoderm inducers BMP4, FGF2 and Activin A promote the generation of populations highly enriched in $\mathrm{KDR}^{+} \mathrm{CD} 235 \mathrm{a} / \mathrm{b}^{+}$mesoderm. Second, I demonstrated that the primitive hematopoietic program transitions through a HEC intermediate. Third, I uncovered previously unappreciated regulatory functions for NOTCH signaling and MYB in the development of the human primitive hematopoietic program. Fourth, I identified a second population of $\mathrm{KDR}^{+} \mathrm{CD} 235 \mathrm{a} / \mathrm{b}^{+}$-derived HECs that emerged following the development of primitive hematopoietic progenitors. These HECs give rise to multipotent hematopoietic progenitors that are phenotypically distinct from the hematopoietic progenitors of the primitive hematopoietic program and generate the NK cell, $\mathrm{T}$ lymphoid, myeloid and erythroid lineages. Looking forward, I have also established a lentivirusbased strategy to determine whether a common mesoderm gives rise to the primitive and MPP 
programs, similar to the common origin of the mouse primitive and EMP programs (Huber et al., 2004; Kennedy et al., 1997).

\subsection{Discussion}

The yolk sac hematopoietic programs were long thought to function solely to support the developing embryo. It is now clear that this is not the case and that populations of tissue-resident macrophages, mast cells and $\mathrm{T}$ lymphocytes that are derived from progenitors of these programs persist in the adult and function to maintain tissue homeostasis (Epelman et al., 2014; Gentek et al., 2018a; Gentek et al., 2018b; Ginhoux et al., 2010; Gomez Perdiguero et al., 2015; Hashimoto et al., 2013; Li et al., 2018; Schulz et al., 2012). Because the progenitors of these immune cell lineages are limited to early stages of development, hPSCs represent the only source to access these clinically relevant populations for further study. In order to successfully generate these progenitors from hPSCs, it is necessary to understand the lineage relationships between the progenitors of the yolk sac hematopoietic programs and the pathways that regulate their specification.

\subsubsection{Yolk sac hematopoietic development}

Our current understanding of yolk sac hematopoiesis is largely derived from work in model organisms and PSC differentiation cultures. Originally defined as a source of erythroid progenitors to sustain the rapid growth of the embryo, it is now clear that this tissue has much broader potential and is able to generate multiple hematopoietic lineages organized in distinct hematopoietic programs. The primitive hematopoietic program is the first to emerge and is transient. It is also the most lineage restricted, generating erythroid, mast cell, macrophage and megakaryocytic progeny. Prior to the exhaustion of the primitive hematopoietic program, a second program, the EMP program emerges from the yolk sac that generates granulocytes (neutrophils), in addition to the erythroid, mast cell, macrophage and megakaryocytic lineages. At the same time, lymphoid progenitors are also detected from what is now referred to as the LMP program. The consensus is that the specification of these programs is restricted to the yolk sac, prior to the emergence of HSCs. As is the case in the definitive hematopoietic program, evidence suggests that all of the yolk sac hematopoietic lineages transition through HECs. With respect to their lineage relationships, the primitive and EMP programs are linked by a common mesoderm, the hemangioblast, while their relationships with the LMP program remains unknown. 
As the human yolk sac is inaccessible, we rely on the hPSC differentiation system to understand these early hematopoietic programs. Several studies have demonstrated that primitive hematopoiesis can be specified from hPSCs, validating that it is possible to model human yolk sac development in vitro. My PhD project is built on the findings of previous work from our lab that showed that human primitive hematopoiesis is derived from $\mathrm{KDR}^{+} \mathrm{CD} 235 \mathrm{a} / \mathrm{b}^{+}$mesoderm, which is distinct from the mesoderm that gives rise to definitive hematopoiesis (Sturgeon et al., 2014). This enabled the separation of the two programs early in development, the in vitro equivalent to dissecting the yolk sac away from the embryo proper. With these tools at hand, I set out to test the hypothesis that human yolk sac hematopoiesis shows similar complexity to that of the mouse and that all of its lineages are specified from $\mathrm{KDR}^{+} \mathrm{CD} 235 \mathrm{a} / \mathrm{b}^{+}$mesoderm.

With appropriately patterned mesoderm, I was able to show that the emergence of human yolk sac hematopoiesis shares many of its features in the mouse, beginning with a primitive hematopoietic program, followed by an MPP program that represents the sum of the EMP and LMP programs. The temporal organization of these programs recapitulates their emergence from the mouse yolk sac, even when isolated from mesoderm. These observations provide further evidence that PSC differentiation recapitulates in vivo development and enables future studies on the regulatory pathways that control these programs. A lineage generated from the mouse yolk sac hematopoiesis that is missing from my work is the B cell lineage (Godin et al., 1995; Yoshimoto et al., 2011). Our lab has developed an assay to monitor B cell potential that has been adapted from protocols to measure this potential from fetal liver and cord blood populations (Xiaoning Sun, unpublished). Unlike definitive hematopoietic populations, the culture of cells of the MPP program with MS-5 stromal cells has failed to generate B cells. It is possible that the frequency of B cell progenitors in these populations are low and that the progenitors cannot be detected at the cell doses assayed. Alternatively, these findings may indicate that essential signaling for human yolk sac B cell development is missing from the culture conditions that are used to generate B cells from hPSCderived definitive populations and $\mathrm{CD} 34^{+}$cord blood progenitors. Finally, it is possible that this population simply does not have B cell potential. While limiting dilution analyses to enumerate the frequency of B cell progenitors in yolk sac have not been reported, less than $60 \%$ of the yolk sacs tested were able to give rise to B cells (Yoshimoto et al., 2011) supporting the notion that this is a rare progenitor population that may require distinct signaling from what is currently delivered. Given that the specific cytokines that drive embryonic B cell development are unknown, one 
strategy to assay the potential of MPP hematopoiesis may be to transplant the cells into the peritoneal cavities of immunodeficient mice, a strategy that has been successfully used to generate B cells from mouse yolk sac and P-Sp progenitors (Yoshimoto et al., 2011).

My analyses also showed that the MPPs fail to engraft following intrahepatic transplantation into immunodeficient neonatal mice, but display an identical surface marker expression pattern to HSCs. The demonstration that the mouse EMP program is sufficient to ensure the survival of the embryo up to birth (Miller et al., 2002) raises the possibility that human yolk sac-derived MPPs are present in "HSC" populations isolated from the fetal liver and cord blood. The presence of the derivatives of these embryonic hematopoietic programs would complicate the interpretation of in vitro assays of lineage potential and molecular analyses. For example, the identification of two clusters in single cell RNA sequencing (scRNA-seq) experiments from a "pure" population may be interpreted as HSCs existing in distinct states. With the possibility of MPP contamination, these clusters may simply represent the progeny of two different embryonic hematopoietic programs. The identification of markers that are specific to the cells of the yolk sac MPP and definitive hematopoietic programs in $\mathrm{CD} 34^{+} \mathrm{CD} 45^{+} \mathrm{CD} 90^{+} \mathrm{CD} 7^{-}$populations would facilitate further characterization of the different cells from fetal liver and cord blood. Previous studies in our lab showed that the definitive hematopoietic cells are produced from multipotent HECs (Ditadi et al., 2015) and offers a source of the earliest definitive hematopoietic cells to also use in these analyses.

The identification of the MPP in the equivalent of what I propose is human yolk sac hematopoiesis suggests that a comparable progenitor may also be present in the mouse. If it exists, the cell may be the progenitor of the EMP and LMP programs. This interpretation is supported by the similar emergence of these programs in the yolk sac (McGrath et al., 2015; Yoshimoto et al., 2012). The detection of the mouse yolk sac MPP may be challenging due to the kinetics of its development, its abundance or the identification of culture conditions that not only promote its proliferation, but also maintain the lineage potential of its progeny. This was the case with human MPPs, as maintenance of the lineage potential of their progeny was dependent upon appropriate stromal cell support.

The demonstration that the BMP4, FGF2 and Activin A-induced $\mathrm{KDR}^{+} \mathrm{CD} 235 \mathrm{a} / \mathrm{b}^{+}$mesoderm has $\mathrm{T}$ lymphoid potential is in contrast to the findings in earlier studies that showed that $\mathrm{KDR}^{+} \mathrm{CD} 235 \mathrm{a} / \mathrm{b}^{+}$mesoderm induced with BMP4, FGF2 and IWP2 do not display this potential 
(Sturgeon et al., 2014). These differences may reflect the fact that the two protocols generate different subsets of $\mathrm{KDR}^{+} \mathrm{CD} 235 \mathrm{a} / \mathrm{b}^{+}$mesoderm with different potentials or that the Tlymphoidcompetent stages were missed in the earlier study. Alternatively, it is possible that IWP2 induces a cell population that represses NOTCH-dependent $\mathrm{T}$ lymphopoiesis. Support for this interpretation is provided by the observation that the IWP2-induced $\mathrm{KDR}^{+} \mathrm{CD} 235 \mathrm{a} / \mathrm{b}^{+}$mesoderm expresses NUMB, an inhibitor of NOTCH signaling (Creamer et al., 2017). Future studies, such as scRNA-seq will investigate the heterogeneity within these populations to help to clarify these discordant $\mathrm{T}$ lymphoid potentials.

With access to different developmental stages of hPSC differentiation, I showed that both the primitive and MPP programs transition through a HEC intermediate, similar to what is found in the mouse (Lancrin et al., 2009; Nishikawa et al., 1998a; Nishikawa et al., 1998b). This work, together with studies of definitive hematopoiesis (Ditadi et al., 2015; Kennedy et al., 2012; Uenishi et al., 2018) provide strong evidence that all of the hematopoietic programs develop from HECs. Currently, it is not possible to distinguish these different populations by surface phenotype, as they all share the same markers. However, access to them provides an opportunity use scRNA-seq or antibody screening approaches to identify program-specific HEC markers.

The demonstration that the primitive and EMP programs are derived from the hemangioblast in the mouse (Huber et al., 2004; Kennedy et al., 1997) and my findings that shows that the EMP hematopoiesis is part of a broader MPP program, suggests that the human hemangioblast initiates all of yolk sac hematopoiesis. To begin to answer this question, I have established a viral markingbased strategy to track clones using a similar method to what has been used to measure the potential of single hematopoietic progenitors in mice and humans (Biasco et al., 2016; Dick et al., 1985; Keller et al., 1985; Keller and Snodgrass, 1990; Scala et al., 2018; Sugimura et al., 2017). If these analyses show that human primitive and MPP hematopoiesis originate from a common yolk sac hematopoietic mesoderm, it raises interesting questions about when and how these programs diverge.

\subsubsection{Function of yolk sac-derived hematopoietic cells}

The yolk sac gives rise to populations with a broad range of lineage potentials, many of which show differences from those produced from the definitive hematopoietic program. Some of these differences reflect the unique needs of the developing embryo, such as its demand for oxygen, 
while other differences indicate that these cells function beyond early stages of embryogenesis. Lineage tracing and transplantation studies have indeed shown that the yolk sac is a source of specialized tissue-resident immune cells that seed different organs where they persist and function throughout life (Epelman et al., 2014; Gentek et al., 2018a; Gentek et al., 2018b; Ginhoux et al., 2010; Gomez Perdiguero et al., 2015; Hashimoto et al., 2013; Li et al., 2018; Schulz et al., 2012). Many of the adult tissues have a balance between the yolk sac and marrow-derived cells. Although the complete function of these cells is not fully understood, studies using model organisms have shown that these yolk sac-derived cells play a pivotal role in organ regeneration (Aurora et al., 2014; Dick et al., 2019; Lavine et al., 2014). The work presented in this thesis enables the research community to access these progenitor populations that would otherwise be unattainable, to further interrogate the function of tissue-resident immune cells. Although the regulatory mechanisms that guide tissue-resident macrophage specification are largely unknown, it is clear that environmental factors direct macrophages to their tissue-specific fates (Gosselin et al., 2014; Lavin et al., 2014). This concept has led to the creation of protocols to generate microglia from hPSCs through the culture of macrophage progenitors with cells of the nervous system (Haenseler et al., 2017; Pandya et al., 2017) or in the presence of cytokines and growth factors known to be present in the brain (Abud et al., 2017; Douvaras et al., 2017; Muffat et al., 2016). A similar approach can be undertaken to generate other populations of tissue-resident macrophages and to identify the regulatory mechanisms that guide their specification. The inclusion of macrophages in protocols that differentiate other cell types from hPSCs may also facilitate their maturation, given their role in tissue morphogenesis in the mouse (DeFalco et al., 2014).

Populations of dermal mast cells are also generated from the yolk sac hematopoietic programs and display a different expression pattern of metabolic enzymes and cytokines than those derived from HSCs (Gentek et al., 2018a). HSC-derived dermal mast cells also uniquely express the Fc receptor, FceR1 and express higher levels of MHCII than their yolk sac progenitor-derived counterpart (Gentek et al., 2018a; Li et al., 2018). These findings indicate that yolk sac-derived mast cells are less inflammatory than those generated from HSCs and raises the possibility that other mast cell subpopulations also develop from progenitors that originate in the yolk sac. Further characterization of the functional differences between yolk sac and definitive-derived mast cells may provide insights into the pathogenesis of mast cell diseases, including mast cell activation 
syndromes, conditions in which mast cell hyper-activation affects the function of multiple organs (reviewed in Akin, 2017).

Studies in the mouse demonstrated that ${\mathrm{V} \gamma 3^{+}}^{+} \mathrm{T}$ lymphocytes emerge early in development and contribute to the DETC population in the adult skin. This cell population persists throughout life, with minimal contribution from HSC-derived progeny (Gentek et al., 2018b; Havran and Allison, 1988, 1990). The demonstration that the yolk sac gives rise to ${\mathrm{V} \gamma 3^{+}}^{+} \mathrm{T}$ lymphocytes following culture with OP9-DL1 cells (Yoshimoto et al., 2012) suggests that this lineage is generated as part of the LMP program. In human development, $\mathrm{V} \gamma 9^{+} \mathrm{V} \delta 2^{+} \mathrm{T}$ lymphocytes dominant the $\mathrm{T}$ cell repertoire at early stages of development (Dimova et al., 2015; Haynes and Heinly, 1995; Haynes et al., 1988b; McVay and Carding, 1996) indicating that they represent the equivalent of mouse $\mathrm{V} 3^{+}$cells. In my thesis work, I was able to show that the MPP population gives rise to both $\gamma \delta$ and $\alpha \beta$ T lymphocytes, which is consistent with potential of the E9.5 mouse yolk sac (Yoshimoto et al., 2012). Further characterization of the MPP-derived $\gamma \delta \mathrm{T}$ lymphoid lineage will determine whether it contains $\mathrm{V} \gamma 9^{+}$or $\mathrm{V} \delta 2^{+}$cells, an observation that would align it with the mouse $\mathrm{V} \gamma 3^{+} \mathrm{T}$ lymphoid lineage that is of yolk sac origin (Havran and Allison, 1990; Yoshimoto et al., 2012). In addition to its role in the generation of $\mathrm{V} \gamma 3^{+}$cells, the transplantation of $\mathrm{T}$ lymphoid progenitors derived from the E9.5 yolk sac and P-Sp into immunodeficient mice gave rise to distinct patterns of $\mathrm{T}$ lymphocyte reconstitution. This is highlighted by increased splenic FoxP3 ${ }^{+} \mathrm{T}$-reg cells and hepatic $\gamma \delta$ T lymphocytes in mice that received the yolk sac-derived cells (Yoshimoto et al., 2012). These data, together with the observation that T-reg cells are abundant in the human fetus (Michaelsson et al., 2006) suggest that the yolk sac is a source of this regulatory lymphoid population. I also showed that MPP-derived $\mathrm{CD} 77^{+} \mathrm{CD} 34^{+} \mathrm{T}$ lymphoid progenitors differ in their expression of CD5 from cord blood-derived cells possibly identifying the earliest stage that the yolk sac and HSC-derived T lymphoid lineages diverge. The recent publication of scRNA-seq datasets of the human fetal and adult thymus (Park et al., 2020; Zeng et al., 2019) will allow for comparative analyses to the hPSC-derived T lymphoid progenitors to be undertaken. Although the samples from these molecular studies are from developmental stages where HSCs have already emerged from the AGM, it is possible that specific subpopulations originated from yolk sac progenitors. If this is the case, the MPP-derived cells would cluster with in vivo populations distinct from those generated from cord blood cells. Likewise, $\mathrm{T}$ lymphoid progenitors generated from the definitive hematopoietic differentiation protocol may cluster with in vivo populations 
differentiated from fetal HSCs. Transcriptomic studies of human $\mathrm{CD}^{+} \mathrm{T}$ lymphocytes have also identified differences between fetal and adult $\mathrm{T}$ lymphopoiesis (Mold et al., 2010) providing a resource to compare the end-stage populations generated in vitro. T lymphocytes are likely not the only adult lymphoid population that develops from yolk sac progenitors. Lineage tracing of mouse Csf1r-expressing cells have shown that E9.5 progenitors give rise to populations of NK cells in the liver at E15.5 (Dege et al., 2020) that may well persist into adulthood, as tissue-resident NK cells. Consistent with studies that have shown that other yolk sac-derived tissue-resident immune cells are unique relative to their HSC-derived counterpart, tissue-resident NK cells show a different RNA expression signature and are functionally distinct from HSC-derived conventional NK cells (Peng et al., 2013; Sojka et al., 2014). Future lineage tracing studies should endeavour to answer whether the yolk sac hematopoietic programs give rise to tissue-resident NK cells. Characterization of the NK cells derived from the human MPP and definitive hematopoietic programs would provide a unique lens to study the specification of tissue-resident and conventional NK cells. Likewise, if B cells are generated from the MPP program, I predict that they will represent the B1 population. The identification of these cells would represent an important step, as it would provide an enriched source of cells to study their development and characterize their function.

\subsubsection{Regulation of yolk sac hematopoietic development}

In addition to the spatiotemporal organization and lineage potential, the different cell signaling and transcription factor requirements of the embryonic hematopoietic programs have offered an additional means to distinguish them from one another. Studies in the zebrafish and mouse have shown that Notch is dispensable for the generation of the primitive and EMP programs, but required for definitive hematopoiesis (Bertrand et al., 2010b; Hadland et al., 2004; Kumano et al., 2003). In addition, our group and others have shown that NOTCH is required for the development of the human definitive hematopoietic program from hPSCs (Ditadi et al., 2015; Uenishi et al., 2018). Despite these findings, the requirement for NOTCH in the development of the human primitive hematopoietic program had not been tested. Here, I show that NOTCH is also required for the EHT of primitive HECs indicating that the regulation of this program differs between the mouse and human. These differences highlight the importance of using human models to study human biology. Studies in the mouse have shown that the strength of Notch signaling regulates definitive hematopoietic development (Gama-Norton et al., 2015) raising the possibility that 
different levels of NOTCH signaling are also required to initiate EHT in the human primitive, MPP and definitive HECs. A better understanding of the role of NOTCH signaling in human hematopoietic development will require markers to distinguish the HECs of the different programs from one another and from endothelial cells and genetic tools to ablate the different NOTCH receptors at specific developmental stages.

Similar to Notch signaling, Myb is differentially required for the development of the embryonic hematopoietic programs in model organisms. In the mouse, the EMP, LMP and definitive hematopoietic programs are Myb-dependent. In contrast, the primitive hematopoietic program, including the erythroid, megakaryocytic and macrophage lineages develop in a Myb-independent fashion (Allen et al., 1999; Mucenski et al., 1991; Mukouyama et al., 1999; Tober et al., 2008). Previous studies using MYB-deficient hESCs have shown that MYB is required for hematopoiesis (Buchrieser et al., 2017; Vanhee et al., 2015). However, as the hematopoietic programs were not characterized in these studies, it was not clear how these defects related to those observed in the mouse. In this study, I showed that MYB is required for the development of the human primitive erythroid and mast cell lineages. Consistent with the finding in the mouse (Schulz et al., 2012), MYB was not required for the generation of macrophages. Together, these observations again identify differences in the regulation of mouse and human hematopoiesis. With the identification of the human MPP program, future studies can be initiated to determine whether the MPP macrophage lineage can also develop in the absence of MYB or if MYB-independent macrophages are solely the product of the primitive hematopoietic program.

Studies in the mouse have shown that the primitive hematopoiesis develops normally in the absence of Raldh2-mediated RA signaling (Goldie et al., 2008) indicating that the human primitive hematopoietic program should not express RALDH2. Consistent with this concept, I showed that the progenitor populations of the primitive and MPP programs lacked Aldefluor activity, which measures the enzymatic activity of RALDH2 and all other ALDHs. In contrast, analyses of populations generated from our definitive hematopoietic differentiation protocol showed that the definitive mesoderm and HEC populations contain $\mathrm{ALDH}^{+}$cells. Studies in our lab have shown that RALDH2 is responsible for the ALDH activity in the definitive lineage and are now focused on determining its responsiveness to RA signaling (Nestor Fernandez, unpublished), as this pathway is required to pattern definitive hematopoiesis in the mouse (Chanda et al., 2013). Interestingly, I found that CYP26A1, the enzyme responsible for converting RA to inactive 
metabolites, is expressed in yolk sac mesoderm and indicates that the yolk sac hematopoietic programs are safeguarded from RA signaling through the expression of this enzyme. There is evidence for this in zebrafish, where regions of the developing heart express cyp26al to restrict RA signaling (Rydeen and Waxman, 2014). My findings are similar to those of studies on the development of the cardiac lineages from hPSCs that showed that the atrial cardiomyocyte lineage develops from $\mathrm{ALDH}^{+}\left(\mathrm{RALDH}^{+}\right)$mesoderm in an RA-dependent fashion. In contrast, the ventricular lineage develops from $\mathrm{CD} 235 \mathrm{a} / \mathrm{b}^{+}$mesoderm, expresses $C Y P 26 A 1$ and does not respond to ROH (Lee et al., 2017). As is the case with hematopoietic development, cardiogenesis also proceeds in distinct waves. The progenitors that give rise to ventricular cardiomyocytes are specified first, while progenitors with atrial cardiomyocyte potential appear at later developmental stages (Lescroart et al., 2014). It is possible that yolk sac and definitive subsets of mesoderm are regulated through similar mechanisms that guide the specification of the ventricular and atrial subsets of mesoderm, respectively. Evidence for this is provided by the observation that yolk sac hematopoietic mesoderm is patterned with similar levels of BMP4, FGF2 and Activin A to what is required to generate ventricular cardiomyocyte mesoderm (Lee et al., 2017). It remains to be seen whether the definitive hematopoietic program can be induced under similar conditions to those used to specify atrial mesoderm.

\subsubsection{Clinical applications}

A landmark study that specifically induced a BRAF mutation in Csflr-expressing cells at E8.5 showed that dysfunctional yolk sac progenitors can cause disease in microglia, as these mice developed neurodegenerative disease (Mass et al., 2017). Because the yolk sac hematopoietic progenitors give rise to tissue-resident immune cells, it is possible that other diseases are also caused by mutations in these embryonic progenitors. Analyses of hematopoietic differentiation of iPSCs generated from individuals with trisomy 21 showed enhanced primitive erythropoiesis, at the expense of the megakaryocytic and myeloid lineages (Chou et al., 2012). Likewise, the frequency of megakaryocyte colony-forming progenitors was increased in EMP-like populations generated from trisomy 21 iPSCs (Maclean et al., 2012). These studies indicate that dysregulated hematopoietic cells derived from the yolk sac programs may be responsible for pediatric cases of leukemia in individuals with Down's syndrome. Furthermore, the demonstration that hPSCs with the ETV6-RUNX1 fusion, which is common to pediatric B cell leukemias (Shurtleff et al., 1995), fail to complete B cell development (Boiers et al., 2018) suggests that a subset of these leukemias 
may also have a yolk sac progenitor origin. The onset of hematologic malignancies that develop from the progeny of the yolk sac programs may not be restricted to the pediatric setting. Primary cutaneous $\gamma \delta$ T cell lymphomas (PCGDTLs) are an aggressive class of adult lymphomas that are derived from the $\gamma \delta$ T lymphoid lineage (reviewed in Tripodo et al., 2009). Of the distinct subtypes of PCGDTLs, the panniculitic subtype is generated from malignant $\mathrm{V} \delta 2^{+}$cells and has a worse prognosis than other PCGDTLs (Daniels et al., 2020). Because V $\delta 2^{+} \mathrm{T}$ lymphocytes are specified at early stages of human development (Haynes and Heinly, 1995; Haynes et al., 1988b; McVay and Carding, 1996), it is possible that mutations in yolk sac-derived progenitors accumulate over the life of the human and eventually cause this cancer. Analyses of the T lymphoid lineage that is generated from the MPP program may facilitate the identification of regulatory mechanisms that would render the malignant cells susceptible to therapy. The MPP-derived T lymphoid cells may also have therapeutic potential, as $\mathrm{V} \gamma 9^{+} \mathrm{V} \delta 2^{+} \mathrm{T}$ lymphocytes isolated from the peripheral blood of adults are able to efficiently kill different types of cancer cells in vitro and in vivo (reviewed in Hoeres et al., 2018). Likewise, the inclusion of T-reg cells derived from the MPP program in the setting of organ transplantation may help to induce tolerance and limit the broad use of immunosuppressive agents in patients post-transplantation (reviewed in Raffin et al., 2020).

While the above studies describe how the derivatives of the yolk sac hematopoietic programs lead to several diseases, the loss of these populations also has negative consequences. Clonal hematopoiesis of indeterminant potential (CHIP) is the result of the progressive dominance of a hematopoietic clone in an individual that lacks other hematologic abnormalities (reviewed in Jaiswal and Ebert, 2019). While CHIP is associated with the risk of future hematologic malignancy, individuals with CHIP are also predisposed to develop cardiovascular disease. The dominant hematopoietic clone in individuals with CHIP commonly has mutations in TET2 (Genovese et al., 2014; Jaiswal et al., 2014). Interestingly, bone marrow transplantation of mouse Tet2-deficient donor cells increased atherosclerotic lesions in the aorta in recipients (Jaiswal et al., 2017) indicating that HSC derivatives promote disease. Analyses of the macrophages in the hearts of recipient mice revealed that the Tet2-null macrophages expressed cytokines associated with inflammation, including $I l l b$ and $I l 6$ (Jaiswal et al., 2017). The accumulation of mutant HSCderived macrophages may be at the expense of the yolk sac-derived tissue-resident cardiac macrophage population that normally maintains tissue homeostasis. This raises the possibility that the transplantation of macrophages generated from the human primitive and MPP programs will 
help minimize cardiovascular disease risk in individuals with CHIP by dampening the proinflammatory response of the mutant macrophages.

The transplantation of yolk sac-derived macrophages may also benefit patients with other cardiac pathologies. These cells have therapeutic potential, as indicated by the demonstration that yolk sac-derived cardiac macrophages are required for myocardial regeneration in the neonatal mouse heart after injury (Aurora et al., 2014). This lineage has also been shown to benefit the adult, as it limits the fibrosis that follows myocardial infarction (MI) (Dick et al., 2019; Lavine et al., 2014). This suggests that the transplantation of macrophages with or without cardiomyocytes may improve outcomes in patients following an MI. The applications of yolk sac-derived macrophages are not limited to the heart. Studies using a mouse model of liver disease showed that the transplantation of hESC-derived macrophages significantly reduces fibrosis and activates the hepatic regenerative response (Haideri et al., 2017). These findings have led to a clinical trial in patients with liver cirrhosis using macrophages differentiated from peripheral blood monocytes (Moroni et al., 2019). Future studies should address whether the transplantation of yolk sac-derived macrophages would offer these patients greater therapeutic benefit. Access to yolk sac-derived macrophages also offers the opportunity to establish novel disease models. This has been pioneered in the brain, in studies in which where hPSC-derived microglia are transplanted into the brains of immunodeficient mice to model Alzheimer's disease (Hasselmann et al., 2019; Mancuso et al., 2019).

\subsection{Future directions}

While my work maps the development of the human hematopoietic programs that originate in the yolk sac, it raises additional questions about the lineage relationships between the primitive and MPP programs. I have established the tools required to test whether the primitive and MPP programs originate from a common mesoderm (Chapter 4), which can be extended to address clonal relationships between other progenitors of the embryonic hematopoietic system. Should my clonal analysis link primitive and MPP hematopoiesis to a common $\mathrm{KDR}^{+} \mathrm{CD} 235 \mathrm{a} / \mathrm{b}^{+}$mesoderm, it is possible that these two programs also share a HEC origin. To address this question, similar analyses to those described in Chapter 4 may be undertaken, whereby the $\mathrm{KDR}^{+} \mathrm{CD} 34^{+}$population on day 5 of differentiation is transduced with lentivirus prior to integration site analyses in primitive erythroid and $\mathrm{T}$ lymphoid progeny. The absence of shared integration sites between the 
primitive erythroid and $\mathrm{T}$ lymphoid populations would indicate that the primitive and MPP programs have distinct HECs. Thereafter, scRNA-seq analyses of the $\mathrm{KDR}^{+} \mathrm{CD} 34^{+}$population may help to identify putative markers and regulatory mechanisms that differ between the two HEC populations. Alternatively, the observation of shared integration sites between the primitive erythroid and $\mathrm{T}$ lymphoid populations would indicate that the primitive and MPP programs develop from a common HEC. In this case, scRNA-seq analyses of the day $5 \mathrm{KDR}^{+} \mathrm{CD} 34^{+}$ population and the day $6 \mathrm{CD} 43^{+}$and $\mathrm{CD} 34^{+} \mathrm{CD} 43^{-}$populations may facilitate the identification of factors that regulate the balance between EHT to generate primitive hematopoietic progenitors and HEC maintenance that go on to give rise to MPPs. This would enable the creation of directed differentiation protocols that generate either the hematopoietic progenitors of the primitive or MPP programs from their common HEC. A shared HEC origin of these programs would be unique to yolk sac hematopoiesis, as the HECs of the mouse EMP and definitive hematopoietic programs are distinct (Chen et al., 2011) and the HECs of the human definitive hematopoietic program develop from a different population of mesoderm (Sturgeon et al., 2014).

The lentivirus-based clonal analyses are not limited to the study of the relationships between the progenitors that give rise to the different hematopoietic programs. Clonal analyses in the mouse and human have demonstrated that the hemangioblast is a common progenitor for the hematopoietic and endothelial lineages (Choi et al., 1998; Huber et al., 2004; Kennedy et al., 2007). In contrast, clonal analyses of hematovascular populations isolated from the mouse or generated from hPSCs have shown that HECs and endothelial cells are distinct cell types (Ditadi et al., 2015; Swiers et al., 2013). However, these analyses were undertaken at stages that closely precede EHT and allow for the possibility that the hematopoietic and endothelial fates diverge immediately prior to EHT. This is supported by clonal analyses of $\mathrm{Flk} 1^{+} \mathrm{CD} 144^{+}$cells isolated at early stages of $\mathrm{mESC}$ differentiation, whereby hematopoietic and endothelial progeny were generated from single cells (Nishikawa et al., 1998a). Here, lentivirus-based clonal analyses of hematopoietic and endothelial potential of the day $4 \mathrm{KDR}^{+} \mathrm{CD} 235 \mathrm{a} / \mathrm{b}^{+}$, day $5 \mathrm{KDR}^{+} \mathrm{CD} 34^{+}$and day $6 \mathrm{CD} 34^{+} \mathrm{CD} 43^{-}$ populations will help to identify the stage and population that contains lineage-restricted progenitors. Thereafter, scRNA-seq analyses will facilitate the identification of markers that distinguish the lineage-committed progenitors from one another and nominate signaling pathways that differentially regulate the balance between the hematopoietic and endothelial fates. This would 
improve our ability to generated hematopoietic cells from hPSCs through the stage-specific manipulation of these pathways with cytokines or small molecules.

While the relationship between the hematopoietic and endothelial lineages is the subject of intense investigation, my work also raises questions of the relationship between the hematopoietic and cardiac fates. I have now demonstrated that both the primitive and MPP programs are generated under similar conditions to those used to specify CD235a/b $/ \mathrm{b}^{+}$mesoderm fated to ventricular cardiomyocyte lineage (Lee et al., 2017). These studies raise two possibilities. One, the hematopoietic and cardiomyocyte lineages develop from separate, but indistinguishable $\mathrm{CD} 235 \mathrm{a} / \mathrm{b}^{+}$progenitors or two, these lineages originate from a common progenitor. Analyses in the mouse have indicated that these lineages may be linked to a common cell. Ablation of Scl, while essential for hematopoietic development (Robb et al., 1995; Shivdasani et al., 1995), leads to the ectopic cardiogenesis in the yolk sac of E9.25 mice (Van Handel et al., 2012). In addition, studies of mESC differentiation have demonstrated that $S c l$ is expressed at early stages of BLCFC-derived blast colony development (D'Souza et al., 2005). Taken together, these studies indicate that $\mathrm{Scl}$ directs the hematopoietic commitment of the BL-CFC at the expense of the cardiac lineage. Lineage commitment may be controlled through Notch signaling, as the prolonged activation of Notch4 in $\mathrm{Flk}^{+}{ }^{+} \mathrm{Bry}^{+}$mesoderm promoted the development of cardiac colonies at the expense of blast colonies. In contrast, transient activation of Notch4 led to the development of mixed hematopoietic and cardiac colonies (Chen et al., 2008) and further suggests that these lineages develop from a common mesoderm. I have preliminary data that shows that cultures induced with BMP4, FGF2 and Activin A also have cardiomyocyte potential (data not shown), an important first step to address whether the hematopoietic and cardiomyocyte fates develop from a common mesoderm. If isolated CD235a $/ \mathrm{b}^{+}$mesoderm gives rise to both lineages, lentivirus-based clonal analyses will help identify whether they originate from a common progenitor. Alternatively, analyses of the lineages contained within single colonies generated from CD235a/b cells in methylcellulose that promotes hematopoietic and cardiomyocyte differentiation, may be undertaken to address this question. Should these lineages emerge from a shared ancestor, the development of methods to selectively promote hematopoietic or cardiomyocyte differentiation would offer significant benefit to biotechnological processes that seek to efficiently generate either lineage in isolation. Similarly, the identification of markers that distinguish lineage-committed 
subsets of $\mathrm{CD} 235 \mathrm{a} / \mathrm{b}^{+}$mesoderm would offer a method to deplete cultures of contaminating populations.

In addition to questions related to the lineage relationships, my study also provides the basis to study the potential function and applications of the hematopoietic cells that are generated from the primitive and MPP programs. Flow cytometric analyses of TCR V $\gamma$ and V $\delta$, and FoxP3 expression of T lymphocytes generated from hPSC-derived MPPs and definitive progenitors, as well as those from cord blood progenitors will provide an indication of whether the developmental differences observed in the mouse and human are preserved in vitro. It is possible that the environment that is provided in vitro is insufficient to generate or sustain the $\gamma \delta$ and T-reg cell lineages. In addition, the in vitro culture system cannot be used to evaluate the site-specific homing of the different $\mathrm{T}$ lymphoid subpopulations generated from MPPs or definitive progenitors. These questions can only be addressed using transplantation models. Previous studies have shown that cord blood-derived T lymphoid progenitors engraft the thymus of immunodeficient neonatal mice (Awong et al., 2009) indicating that this model may provide an approach to address the question of homing. If the MPP programs represents the dominant source of $\mathrm{V} \gamma 9^{+} \mathrm{V} \delta 2^{+} \mathrm{T}$ lymphocytes or T-reg cells, I would expect to find these cells in the skin and spleen of the engrafted mice. These findings would support the interpretation that they are generated from the yolk sac and demonstrate the BMP4, FGF2 and Activin A-induced mesoderm provides a novel source of these cells for future studies and potential therapeutic applications.

The transplantation of macrophage progenitors generated from primitive and MPP hematopoiesis may also enable the generation of different tissue-resident macrophage subsets in vivo. While immunodeficient mice are receptive of human hematopoietic cells, macrophage development in these animals is poor. This is likely because the human cells do not efficiently bind mouse M-csf through their receptor, CSF1R. In support of this possibility, Rathinam et al. (2011) showed that expression of human CSF1 (M-CSF) in these mice significantly improved the level of monocyte/macrophage engraftment following the transplantation of CD $34^{+}$cord blood cells. The use of this model or variants of it have been used to study hPSC-derive microglia (Abud et al., 2017; Hasselmann et al., 2019; Mancuso et al., 2019) highlighting the viability of this strategy. Molecular analyses, such as scRNA-seq will help to determine whether the engrafted macrophages adopt tissue-resident fates when compared to published datasets of tissue-resident macrophages isolated from the human. The demonstration that these progenitors can differentiate to tissue- 
resident macrophage fates will be an important step in determining what regulates their development and function. 


\section{References}

Abud, E.M., Ramirez, R.N., Martinez, E.S., Healy, L.M., Nguyen, C.H.H., Newman, S.A., Yeromin, A.V., Scarfone, V.M., Marsh, S.E., Fimbres, C., et al. (2017). iPSC-Derived Human Microglia-like Cells to Study Neurological Diseases. Neuron 94, 278-293.e279.

Akin, C. (2017). Mast cell activation syndromes. J Allergy Clin Immunol 140, 349-355.

Allen, R.D., 3rd, Bender, T.P., and Siu, G. (1999). c-Myb is essential for early T cell development. Genes Dev 13, 1073-1078.

Alliot, F., Godin, I., and Pessac, B. (1999). Microglia derive from progenitors, originating from the yolk sac, and which proliferate in the brain. Brain Res Dev Brain Res 117, 145-152.

Alzubi, J., Pallant, C., Mussolino, C., Howe, S.J., Thrasher, A.J., and Cathomen, T. (2017).

Targeted genome editing restores $\mathrm{T}$ cell differentiation in a humanized X-SCID pluripotent stem cell disease model. Sci Rep 7, 12475.

Amabile, G., Welner, R.S., Nombela-Arrieta, C., D'Alise, A.M., Di Ruscio, A., Ebralidze, A.K., Kraytsberg, Y., Ye, M., Kocher, O., Neuberg, D.S., et al. (2013). In vivo generation of transplantable human hematopoietic cells from induced pluripotent stem cells. Blood 121, 12551264.

Antonarakis, S.E. (2017). Down syndrome and the complexity of genome dosage imbalance. Nat Rev Genet 18, 147-163.

Aurora, A.B., Porrello, E.R., Tan, W., Mahmoud, A.I., Hill, J.A., Bassel-Duby, R., Sadek, H.A., and Olson, E.N. (2014). Macrophages are required for neonatal heart regeneration. J Clin Invest 124, 1382-1392.

Awong, G., Herer, E., Surh, C.D., Dick, J.E., La Motte-Mohs, R.N., and Zúñiga-Pflücker, J.C. (2009). Characterization in vitro and engraftment potential in vivo of human progenitor $\mathrm{T}$ cells generated from hematopoietic stem cells. Blood 114, 972-982.

Bai, H., Xie, Y.-L., Gao, Y.-X., Cheng, T., and Wang, Z.Z. (2013). The balance of positive and negative effects of TGF- $\beta$ signaling regulates the development of hematopoietic and endothelial progenitors in human pluripotent stem cells. Stem Cells Dev 22, 2765-2776.

Bain, C.C., Bravo-Blas, A., Scott, C.L., Perdiguero, E.G., Geissmann, F., Henri, S., Malissen, B., Osborne, L.C., Artis, D., and Mowat, A.M. (2014). Constant replenishment from circulating monocytes maintains the macrophage pool in the intestine of adult mice. Nat Immunol 15, 929937.

Balassa, K., Danby, R., and Rocha, V. (2019). Haematopoietic stem cell transplants: principles and indications. Br J Hosp Med (Lond) 80, 33-39.

Barber, C.L., Montecino-Rodriguez, E., and Dorshkind, K. (2011). Reduced production of B-1specified common lymphoid progenitors results in diminished potential of adult marrow to generate B-1 cells. Proc Natl Acad Sci U S A 108, 13700-13704. 
Barker, J.E. (1968). Development of the mouse hematopoietic system. I. Types of hemoglobin produced in embryonic yolk sac and liver. Dev Biol 18, 14-29.

Baumgarth, N. (2017). A Hard(y) Look at B-1 Cell Development and Function. J Immunol 199, 3387-3394.

Becker, A.J., McCulloch, E.A., and Till, J.E. (1963). Cytological demonstration of the clonal nature of spleen colonies derived from transplanted mouse marrow cells. Nature 197, 452-454.

Benveniste, P., Cantin, C., Hyam, D., and Iscove, N.N. (2003). Hematopoietic stem cells engraft in mice with absolute efficiency. Nat Immunol 4, 708-713.

Bernardo, A.S., Faial, T., Gardner, L., Niakan, K.K., Ortmann, D., Senner, C.E., Callery, E.M., Trotter, M.W., Hemberger, M., Smith, J.C., et al. (2011). BRACHYURY and CDX2 mediate BMP-induced differentiation of human and mouse pluripotent stem cells into embryonic and extraembryonic lineages. Cell Stem Cell 9, 144-155.

Bertrand, J.Y., Chi, N.C., Santoso, B., Teng, S., Stainier, D.Y., and Traver, D. (2010a).

Haematopoietic stem cells derive directly from aortic endothelium during development. Nature 464, 108-111.

Bertrand, J.Y., Cisson, J.L., Stachura, D.L., and Traver, D. (2010b). Notch signaling distinguishes 2 waves of definitive hematopoiesis in the zebrafish embryo. Blood 115, 27772783.

Biasco, L., Pellin, D., Scala, S., Dionisio, F., Basso-Ricci, L., Leonardelli, L., Scaramuzza, S., Baricordi, C., Ferrua, F., Cicalese, M.P., et al. (2016). In Vivo Tracking of Human Hematopoiesis Reveals Patterns of Clonal Dynamics during Early and Steady-State Reconstitution Phases. Cell Stem Cell 19, 107-119.

Bloom, W., and Bartelmez, G.W. (1940). Hematopoiesis in young human embryos. American Journal of Anatomy 67, 21-53.

Boiers, C., Carrelha, J., Lutteropp, M., Luc, S., Green, J.C., Azzoni, E., Woll, P.S., Mead, A.J., Hultquist, A., Swiers, G., et al. (2013). Lymphomyeloid contribution of an immune-restricted progenitor emerging prior to definitive hematopoietic stem cells. Cell Stem Cell 13, 535-548.

Boiers, C., Richardson, S.E., Laycock, E., Zriwil, A., Turati, V.A., Brown, J., Wray, J.P., Wang, D., James, C., Herrero, J., et al. (2018). A Human IPS Model Implicates Embryonic B-Myeloid Fate Restriction as Developmental Susceptibility to B Acute Lymphoblastic LeukemiaAssociated ETV6-RUNX1. Dev Cell 44, 362-377.e367.

Boisset, J.C., van Cappellen, W., Andrieu-Soler, C., Galjart, N., Dzierzak, E., and Robin, C. (2010). In vivo imaging of haematopoietic cells emerging from the mouse aortic endothelium. Nature 464, 116-120.

Boocock, G.R., Morrison, J.A., Popovic, M., Richards, N., Ellis, L., Durie, P.R., and Rommens, J.M. (2003). Mutations in SBDS are associated with Shwachman-Diamond syndrome. Nat Genet $33,97-101$. 
Boyer, S.W., Schroeder, A.V., Smith-Berdan, S., and Forsberg, E.C. (2011). All hematopoietic cells develop from hematopoietic stem cells through Flk2/Flt3-positive progenitor cells. Cell Stem Cell 9, 64-73.

Brauer, P.M., Pessach, I.M., Clarke, E., Rowe, J.H., Ott de Bruin, L., Lee, Y.N., DominguezBrauer, C., Comeau, A.M., Awong, G., Felgentreff, K., et al. (2016). Modeling altered T-cell development with induced pluripotent stem cells from patients with RAG1-dependent immune deficiencies. Blood 128, 783-793.

Bray, S.J. (2016). Notch signalling in context. Nat Rev Mol Cell Biol 17, 722-735.

Brewer, J.R., Mazot, P., and Soriano, P. (2016). Genetic insights into the mechanisms of Fgf signaling. Genes Dev 30, 751-771.

Brown, C.E., Alizadeh, D., Starr, R., Weng, L., Wagner, J.R., Naranjo, A., Ostberg, J.R., Blanchard, M.S., Kilpatrick, J., Simpson, J., et al. (2016). Regression of Glioblastoma after Chimeric Antigen Receptor T-Cell Therapy. N Engl J Med 375, 2561-2569.

Buchrieser, J., James, W., and Moore, M.D. (2017). Human Induced Pluripotent Stem CellDerived Macrophages Share Ontogeny with MYB-Independent Tissue-Resident Macrophages. Stem Cell Reports 8, 334-345.

Bustelo, X.R., Rubin, S.D., Suen, K.L., Carrasco, D., and Barbacid, M. (1993). Developmental expression of the vav protooncogene. Cell Growth Differ 4, 297-308.

Butko, E., Pouget, C., and Traver, D. (2016). Complex regulation of HSC emergence by the Notch signaling pathway. Dev Biol 409, 129-138.

Carmeliet, P., Ferreira, V., Breier, G., Pollefeyt, S., Kieckens, L., Gertsenstein, M., Fahrig, M., Vandenhoeck, A., Harpal, K., Eberhardt, C., et al. (1996). Abnormal blood vessel development and lethality in embryos lacking a single VEGF allele. Nature 380, 435-439.

Chanda, B., Ditadi, A., Iscove, N.N., and Keller, G. (2013). Retinoic acid signaling is essential for embryonic hematopoietic stem cell development. Cell 155, 215-227.

Chao, M.P., Gentles, A.J., Chatterjee, S., Lan, F., Reinisch, A., Corces, M.R., Xavy, S., Shen, J., Haag, D., Chanda, S., et al. (2017). Human AML-iPSCs Reacquire Leukemic Properties after Differentiation and Model Clonal Variation of Disease. Cell Stem Cell 20, 329-344.e327.

Chen, M.J., Li, Y., De Obaldia, M.E., Yang, Q., Yzaguirre, A.D., Yamada-Inagawa, T., Vink, C.S., Bhandoola, A., Dzierzak, E., and Speck, N.A. (2011). Erythroid/myeloid progenitors and hematopoietic stem cells originate from distinct populations of endothelial cells. Cell Stem Cell 9, 541-552.

Chen, M.J., Yokomizo, T., Zeigler, B.M., Dzierzak, E., and Speck, N.A. (2009). Runx1 is required for the endothelial to haematopoietic cell transition but not thereafter. Nature 457, 887891. 
Chen, V.C., Stull, R., Joo, D., Cheng, X., and Keller, G. (2008). Notch signaling respecifies the hemangioblast to a cardiac fate. Nat Biotechnol 26, 1169-1178.

Choi, K., Kennedy, M., Kazarov, A., Papadimitriou, J.C., and Keller, G. (1998). A common precursor for hematopoietic and endothelial cells. Development 125, 725-732.

Choi, K.D., Vodyanik, M.A., Togarrati, P.P., Suknuntha, K., Kumar, A., Samarjeet, F., Probasco, M.D., Tian, S., Stewart, R., Thomson, J.A., et al. (2012). Identification of the hemogenic endothelial progenitor and its direct precursor in human pluripotent stem cell differentiation cultures. Cell Rep 2, 553-567.

Chou, S.T., Byrska-Bishop, M., Tober, J.M., Yao, Y., Vandorn, D., Opalinska, J.B., Mills, J.A., Choi, J.K., Speck, N.A., Gadue, P., et al. (2012). Trisomy 21 -associated defects in human primitive hematopoiesis revealed through induced pluripotent stem cells. Proc Natl Acad Sci U S A $109,17573-17578$.

Chung, Y.S., Zhang, W.J., Arentson, E., Kingsley, P.D., Palis, J., and Choi, K. (2002). Lineage analysis of the hemangioblast as defined by FLK1 and SCL expression. Development 129, 55115520.

Clevers, H., and Nusse, R. (2012). Wnt/ $\beta$-catenin signaling and disease. Cell 149, 1192-1205.

Conlon, F.L., Lyons, K.M., Takaesu, N., Barth, K.S., Kispert, A., Herrmann, B., and Robertson, E.J. (1994). A primary requirement for nodal in the formation and maintenance of the primitive streak in the mouse. Development 120, 1919-1928.

Conlon, R.A., Reaume, A.G., and Rossant, J. (1995). Notch1 is required for the coordinate segmentation of somites. Development 121, 1533-1545.

Covens, K., Verbinnen, B., Geukens, N., Meyts, I., Schuit, F., Van Lommel, L., Jacquemin, M., and Bossuyt, X. (2013a). Characterization of proposed human B-1 cells reveals pre-plasmablast phenotype. Blood 121, 5176-5183.

Covens, K., Verbinnen, B., Jacquemin, M., and Bossuyt, X. (2013b). Response: Extended analysis of microarray data does not contradict preplasmablast phenotype of human CD20+CD27+CD43+ cells. Blood 122, 3693-3694.

Creamer, J.P., Dege, C., Ren, Q., Ho, J.T.K., Valentine, M.C., Druley, T.E., and Sturgeon, C.M. (2017). Human definitive hematopoietic specification from pluripotent stem cells is regulated by mesodermal expression of CDX4. Blood 129, 2988-2992.

Cumano, A., Ferraz, J.C., Klaine, M., Di Santo, J.P., and Godin, I. (2001). Intraembryonic, but not yolk sac hematopoietic precursors, isolated before circulation, provide long-term multilineage reconstitution. Immunity $15,477-485$.

D'Amour, K.A., Agulnick, A.D., Eliazer, S., Kelly, O.G., Kroon, E., and Baetge, E.E. (2005). Efficient differentiation of human embryonic stem cells to definitive endoderm. Nat Biotechnol 23, 1534-1541. 
D'Souza, S.L., Elefanty, A.G., and Keller, G. (2005). SCL/Tal-1 is essential for hematopoietic commitment of the hemangioblast but not for its development. Blood 105, 3862-3870.

Dai, P., Akimaru, H., Tanaka, Y., Hou, D.X., Yasukawa, T., Kanei-Ishii, C., Takahashi, T., and Ishii, S. (1996). CBP as a transcriptional coactivator of c-Myb. Genes Dev 10, 528-540.

Daniels, J., Doukas, P.G., Escala, M.E.M., Ringbloom, K.G., Shih, D.J.H., Yang, J., Tegtmeyer, K., Park, J., Thomas, J.J., Selli, M.E., et al. (2020). Cellular origins and genetic landscape of cutaneous gamma delta $\mathrm{T}$ cell lymphomas. Nat Commun 11, 1806.

de Andres, B., Gonzalo, P., Minguet, S., Martinez-Marin, J.A., Soro, P.G., Marcos, M.A., and Gaspar, M.L. (2002). The first 3 days of B-cell development in the mouse embryo. Blood 100, 4074-4081.

de Bruijn, M.F., Ma, X., Robin, C., Ottersbach, K., Sanchez, M.J., and Dzierzak, E. (2002). Hematopoietic stem cells localize to the endothelial cell layer in the midgestation mouse aorta. Immunity 16, 673-683.

de Jong, J.L., Davidson, A.J., Wang, Y., Palis, J., Opara, P., Pugach, E., Daley, G.Q., and Zon, L.I. (2010). Interaction of retinoic acid and scl controls primitive blood development. Blood 116, 201-209.

DeFalco, T., Bhattacharya, I., Williams, A.V., Sams, D.M., and Capel, B. (2014). Yolk-sacderived macrophages regulate fetal testis vascularization and morphogenesis. Proc Natl Acad Sci U S A 111, E2384-2393.

Dege, C., Fegan, K.H., Creamer, J.P., Berrien-Elliott, M.M., Luff, S.A., Kim, D., Wagner, J.A., Kingsley, P.D., McGrath, K.E., Fehniger, T.A., et al. (2020). Potently Cytotoxic Natural Killer Cells Initially Emerge from Erythro-Myeloid Progenitors during Mammalian Development. Dev Cell.

Del Amo, F.F., Smith, D.E., Swiatek, P.J., Gendron-Maguire, M., Greenspan, R.J., McMahon, A.P., and Gridley, T. (1992). Expression pattern of Motch, a mouse homolog of Drosophila Notch, suggests an important role in early postimplantation mouse development. Development $115,737-744$.

Deng, C.X., Wynshaw-Boris, A., Shen, M.M., Daugherty, C., Ornitz, D.M., and Leder, P. (1994). Murine FGFR-1 is required for early postimplantation growth and axial organization. Genes Dev 8, 3045-3057.

Descatoire, M., Weill, J.C., Reynaud, C.A., and Weller, S. (2011). A human equivalent of mouse B-1 cells? J Exp Med 208, 2563-2564.

Diamond, L.K., and Blackfan, K.D. (1938). Hypoplastic anemia. Am J Dis Child 56, 464-467.

Dick, J.E., Magli, M.C., Huszar, D., Phillips, R.A., and Bernstein, A. (1985). Introduction of a selectable gene into primitive stem cells capable of long-term reconstitution of the hemopoietic system of W/Wv mice. Cell 42, 71-79. 
Dick, S.A., Macklin, J.A., Nejat, S., Momen, A., Clemente-Casares, X., Althagafi, M.G., Chen, J., Kantores, C., Hosseinzadeh, S., Aronoff, L., et al. (2019). Self-renewing resident cardiac macrophages limit adverse remodeling following myocardial infarction. Nat Immunol 20, 29-39.

Dieterlen-Lievre, F. (1975). On the origin of haemopoietic stem cells in the avian embryo: an experimental approach. J Embryol Exp Morphol 33, 607-619.

Dimova, T., Brouwer, M., Gosselin, F., Tassignon, J., Leo, O., Donner, C., Marchant, A., and Vermijlen, D. (2015). Effector Vgamma9Vdelta2 T cells dominate the human fetal gammadelta T-cell repertoire. Proc Natl Acad Sci U S A 112, E556-565.

Ditadi, A., Sturgeon, C.M., and Keller, G. (2017). A view of human haematopoietic development from the Petri dish. Nat Rev Mol Cell Biol 18, 56-67.

Ditadi, A., Sturgeon, C.M., Tober, J., Awong, G., Kennedy, M., Yzaguirre, A.D., Azzola, L., Ng, E.S., Stanley, E.G., French, D.L., et al. (2015). Human definitive haemogenic endothelium and arterial vascular endothelium represent distinct lineages. Nat Cell Biol 17, 580-591.

Dorey, K., and Amaya, E. (2010). FGF signalling: diverse roles during early vertebrate embryogenesis. Development 137, 3731-3742.

Dou, D.R., Calvanese, V., Sierra, M.I., Nguyen, A.T., Minasian, A., Saarikoski, P., Sasidharan, R., Ramirez, C.M., Zack, J.A., Crooks, G.M., et al. (2016). Medial HOXA genes demarcate haematopoietic stem cell fate during human development. Nat Cell Biol 18, 595-606.

Doulatov, S., Vo, L.T., Macari, E.R., Wahlster, L., Kinney, M.A., Taylor, A.M., Barragan, J., Gupta, M., McGrath, K., Lee, H.Y., et al. (2017). Drug discovery for Diamond-Blackfan anemia using reprogrammed hematopoietic progenitors. Sci Transl Med 9.

Douvaras, P., Sun, B., Wang, M., Kruglikov, I., Lallos, G., Zimmer, M., Terrenoire, C., Zhang, B., Gandy, S., Schadt, E., et al. (2017). Directed Differentiation of Human Pluripotent Stem Cells to Microglia. Stem Cell Reports 8, 1516-1524.

Draptchinskaia, N., Gustavsson, P., Andersson, B., Pettersson, M., Willig, T.N., Dianzani, I., Ball, S., Tchernia, G., Klar, J., Matsson, H., et al. (1999). The gene encoding ribosomal protein S19 is mutated in Diamond-Blackfan anaemia. Nat Genet 21, 169-175.

Dumont, D.J., Yamaguchi, T.P., Conlon, R.A., Rossant, J., and Breitman, M.L. (1992). tek, a novel tyrosine kinase gene located on mouse chromosome 4, is expressed in endothelial cells and their presumptive precursors. Oncogene 7, 1471-1480.

Eilken, H.M., Nishikawa, S., and Schroeder, T. (2009). Continuous single-cell imaging of blood generation from haemogenic endothelium. Nature 457, 896-900.

Epelman, S., Lavine, K.J., Beaudin, A.E., Sojka, D.K., Carrero, J.A., Calderon, B., Brija, T., Gautier, E.L., Ivanov, S., Satpathy, A.T., et al. (2014). Embryonic and adult-derived resident cardiac macrophages are maintained through distinct mechanisms at steady state and during inflammation. Immunity 40, 91-104. 
Fallon, P., Gentry, T., Balber, A.E., Boulware, D., Janssen, W.E., Smilee, R., Storms, R.W., and Smith, C. (2003). Mobilized peripheral blood SSCloALDHbr cells have the phenotypic and functional properties of primitive haematopoietic cells and their number correlates with engraftment following autologous transplantation. Br J Haematol 122, 99-108.

Faloon, P., Arentson, E., Kazarov, A., Deng, C.X., Porcher, C., Orkin, S., and Choi, K. (2000). Basic fibroblast growth factor positively regulates hematopoietic development. Development 127, 1931-1941.

Farley, A.M., Morris, L.X., Vroegindeweij, E., Depreter, M.L., Vaidya, H., Stenhouse, F.H., Tomlinson, S.R., Anderson, R.A., Cupedo, T., Cornelissen, J.J., et al. (2013). Dynamics of thymus organogenesis and colonization in early human development. Development 140, 20152026.

Fehling, H.J., Lacaud, G., Kubo, A., Kennedy, M., Robertson, S., Keller, G., and Kouskoff, V. (2003). Tracking mesoderm induction and its specification to the hemangioblast during embryonic stem cell differentiation. Development 130, 4217-4227.

Ferkowicz, M.J., Starr, M., Xie, X., Li, W., Johnson, S.A., Shelley, W.C., Morrison, P.R., and Yoder, M.C. (2003). CD41 expression defines the onset of primitive and definitive hematopoiesis in the murine embryo. Development 130, 4393-4403.

Ferrara, N., Carver-Moore, K., Chen, H., Dowd, M., Lu, L., O'Shea, K.S., Powell-Braxton, L., Hillan, K.J., and Moore, M.W. (1996). Heterozygous embryonic lethality induced by targeted inactivation of the VEGF gene. Nature 380, 439-442.

Frame, J.M., Fegan, K.H., Conway, S.J., McGrath, K.E., and Palis, J. (2016). Definitive Hematopoiesis in the Yolk Sac Emerges from Wnt-Responsive Hemogenic Endothelium Independently of Circulation and Arterial Identity. Stem Cells 34, 431-444.

Gadue, P., Huber, T.L., Paddison, P.J., and Keller, G.M. (2006). Wnt and TGF-beta signaling are required for the induction of an in vitro model of primitive streak formation using embryonic stem cells. Proc Natl Acad Sci U S A 103, 16806-16811.

Galic, Z., Kitchen, S.G., Kacena, A., Subramanian, A., Burke, B., Cortado, R., and Zack, J.A. (2006). T lineage differentiation from human embryonic stem cells. Proc Natl Acad Sci U S A $103,11742-11747$.

Gama-Norton, L., Ferrando, E., Ruiz-Herguido, C., Liu, Z., Guiu, J., Islam, A.B., Lee, S.U., Yan, M., Guidos, C.J., López-Bigas, N., et al. (2015). Notch signal strength controls cell fate in the haemogenic endothelium. Nat Commun 6, 8510.

Gao, L., Tober, J., Gao, P., Chen, C., Tan, K., and Speck, N.A. (2018). RUNX1 and the endothelial origin of blood. Exp Hematol 68, 2-9.

Gekas, C., Dieterlen-Lievre, F., Orkin, S.H., and Mikkola, H.K. (2005). The placenta is a niche for hematopoietic stem cells. Dev Cell 8, 365-375. 
Genovese, G., Kähler, A.K., Handsaker, R.E., Lindberg, J., Rose, S.A., Bakhoum, S.F., Chambert, K., Mick, E., Neale, B.M., Fromer, M., et al. (2014). Clonal hematopoiesis and bloodcancer risk inferred from blood DNA sequence. N Engl J Med 371, 2477-2487.

Gentek, R., Ghigo, C., Hoeffel, G., Bulle, M.J., Msallam, R., Gautier, G., Launay, P., Chen, J., Ginhoux, F., and Bajenoff, M. (2018a). Hemogenic Endothelial Fate Mapping Reveals Dual Developmental Origin of Mast Cells. Immunity 48, 1160-1171.e1165.

Gentek, R., Ghigo, C., Hoeffel, G., Jorquera, A., Msallam, R., Wienert, S., Klauschen, F., Ginhoux, F., and Bajénoff, M. (2018b). Epidermal $\gamma \delta$ T cells originate from yolk sac hematopoiesis and clonally self-renew in the adult. Journal of Experimental Medicine 215, 29943005.

Ghyselinck, N.B., and Duester, G. (2019). Retinoic acid signaling pathways. Development 146.

Ginhoux, F., Greter, M., Leboeuf, M., Nandi, S., See, P., Gokhan, S., Mehler, M.F., Conway, S.J., Ng, L.G., Stanley, E.R., et al. (2010). Fate mapping analysis reveals that adult microglia derive from primitive macrophages. Science 330, 841-845.

Godin, I., Dieterlen-Lièvre, F., and Cumano, A. (1995). Emergence of multipotent hemopoietic cells in the yolk sac and paraaortic splanchnopleura in mouse embryos, beginning at 8.5 days postcoitus. Proc Natl Acad Sci U S A 92, 773-777.

Goldie, L.C., Lucitti, J.L., Dickinson, M.E., and Hirschi, K.K. (2008). Cell signaling directing the formation and function of hemogenic endothelium during murine embryogenesis. Blood 112, 3194-3204.

Gomez Perdiguero, E., Klapproth, K., Schulz, C., Busch, K., Azzoni, E., Crozet, L., Garner, H., Trouillet, C., de Bruijn, M.F., Geissmann, F., et al. (2015). Tissue-resident macrophages originate from yolk-sac-derived erythro-myeloid progenitors. Nature 518, 547-551.

Gosselin, D., Link, V.M., Romanoski, C.E., Fonseca, G.J., Eichenfield, D.Z., Spann, N.J., Stender, J.D., Chun, H.B., Garner, H., Geissmann, F., et al. (2014). Environment drives selection and function of enhancers controlling tissue-specific macrophage identities. Cell 159, $1327-$ 1340 .

Greber, B., Lehrach, H., and Adjaye, J. (2007). Fibroblast growth factor 2 modulates transforming growth factor beta signaling in mouse embryonic fibroblasts and human ESCs (hESCs) to support hESC self-renewal. Stem Cells 25, 455-464.

Griffin, D.O., Holodick, N.E., and Rothstein, T.L. (2011a). Human B1 cells are CD3-: A reply to "A human equivalent of mouse B-1 cells?" and "The nature of circulating CD27+CD43+ B cells". J Exp Med 208, 2566-2569.

Griffin, D.O., Holodick, N.E., and Rothstein, T.L. (2011b). Human B1 cells in umbilical cord and adult peripheral blood express the novel phenotype CD20+CD27+ CD43+ CD70. J Exp Med 208, 67-80. 
Gurdon, J.B., Harger, P., Mitchell, A., and Lemaire, P. (1994). Activin signalling and response to a morphogen gradient. Nature 371, 487-492.

Haar, J.L., and Ackerman, G.A. (1971). A phase and electron microscopic study of vasculogenesis and erythropoiesis in the yolk sac of the mouse. Anat Rec 170, 199-223.

Hadland, B.K., Huppert, S.S., Kanungo, J., Xue, Y., Jiang, R., Gridley, T., Conlon, R.A., Cheng, A.M., Kopan, R., and Longmore, G.D. (2004). A requirement for Notch1 distinguishes 2 phases of definitive hematopoiesis during development. Blood 104, 3097-3105.

Hadland, B.K., Varnum-Finney, B., Mandal, P.K., Rossi, D.J., Poulos, M.G., Butler, J.M., Rafii, S., Yoder, M.C., Yoshimoto, M., and Bernstein, I.D. (2017). A Common Origin for B-1a and B2 Lymphocytes in Clonal Pre- Hematopoietic Stem Cells. Stem Cell Reports 8, 1563-1572.

Hadland, B.K., Varnum-Finney, B., Poulos, M.G., Moon, R.T., Butler, J.M., Rafii, S., and Bernstein, I.D. (2015). Endothelium and NOTCH specify and amplify aorta-gonadmesonephros-derived hematopoietic stem cells. J Clin Invest 125, 2032-2045.

Haenseler, W., Sansom, S.N., Buchrieser, J., Newey, S.E., Moore, C.S., Nicholls, F.J., Chintawar, S., Schnell, C., Antel, J.P., Allen, N.D., et al. (2017). A Highly Efficient Human Pluripotent Stem Cell Microglia Model Displays a Neuronal-Co-culture-Specific Expression Profile and Inflammatory Response. Stem Cell Reports 8, 1727-1742.

Haideri, S.S., McKinnon, A.C., Taylor, A.H., Kirkwood, P., Starkey Lewis, P.J., O'Duibhir, E., Vernay, B., Forbes, S., and Forrester, L.M. (2017). Injection of embryonic stem cell derived macrophages ameliorates fibrosis in a murine model of liver injury. NPJ Regen Med 2, 14.

Hashimoto, D., Chow, A., Noizat, C., Teo, P., Beasley, M.B., Leboeuf, M., Becker, C.D., See, P., Price, J., Lucas, D., et al. (2013). Tissue-resident macrophages self-maintain locally throughout adult life with minimal contribution from circulating monocytes. Immunity 38, $792-$ 804.

Hasselmann, J., Coburn, M.A., England, W., Figueroa Velez, D.X., Kiani Shabestari, S., Tu, C.H., McQuade, A., Kolahdouzan, M., Echeverria, K., Claes, C., et al. (2019). Development of a Chimeric Model to Study and Manipulate Human Microglia In Vivo. Neuron 103, 10161033.e1010.

Havran, W.L., and Allison, J.P. (1988). Developmentally ordered appearance of thymocytes expressing different T-cell antigen receptors. Nature 335, 443-445.

Havran, W.L., and Allison, J.P. (1990). Origin of Thy-1+ dendritic epidermal cells of adult mice from fetal thymic precursors. Nature 344, 68-70.

Haynes, B.F., and Heinly, C.S. (1995). Early human T cell development: analysis of the human thymus at the time of initial entry of hematopoietic stem cells into the fetal thymic microenvironment. J Exp Med 181, 1445-1458. 
Haynes, B.F., Martin, M.E., Kay, H.H., and Kurtzberg, J. (1988a). Early events in human T cell ontogeny. Phenotypic characterization and immunohistologic localization of T cell precursors in early human fetal tissues. J Exp Med 168, 1061-1080.

Haynes, B.F., Singer, K.H., Denning, S.M., and Martin, M.E. (1988b). Analysis of expression of $\mathrm{CD} 2, \mathrm{CD} 3$, and $\mathrm{T}$ cell antigen receptor molecules during early human fetal thymic development. J Immunol 141, 3776-3784.

Hess, D.A., Meyerrose, T.E., Wirthlin, L., Craft, T.P., Herrbrich, P.E., Creer, M.H., and Nolta, J.A. (2004). Functional characterization of highly purified human hematopoietic repopulating cells isolated according to aldehyde dehydrogenase activity. Blood 104, 1648-1655.

Hoeres, T., Smetak, M., Pretscher, D., and Wilhelm, M. (2018). Improving the Efficiency of Vy9V82 T-Cell Immunotherapy in Cancer. Front Immunol 9, 800-800.

Huang, H., and Auerbach, R. (1993). Identification and characterization of hematopoietic stem cells from the yolk sac of the early mouse embryo. Proc Natl Acad Sci U S A 90, 10110-10114.

Huber, T.L., Kouskoff, V., Fehling, H.J., Palis, J., and Keller, G. (2004). Haemangioblast commitment is initiated in the primitive streak of the mouse embryo. Nature 432, 625-630.

Ivanovs, A., Rybtsov, S., Anderson, R.A., Turner, M.L., and Medvinsky, A. (2014).

Identification of the niche and phenotype of the first human hematopoietic stem cells. Stem Cell Reports 2, 449-456.

Ivanovs, A., Rybtsov, S., Ng, E.S., Stanley, E.G., Elefanty, A.G., and Medvinsky, A. (2017). Human haematopoietic stem cell development: from the embryo to the dish. Development 144, 2323-2337.

Ivanovs, A., Rybtsov, S., Welch, L., Anderson, R.A., Turner, M.L., and Medvinsky, A. (2011). Highly potent human hematopoietic stem cells first emerge in the intraembryonic aorta-gonadmesonephros region. J Exp Med 208, 2417-2427.

Ivarsson, M.A., Loh, L., Marquardt, N., Kekalainen, E., Berglin, L., Bjorkstrom, N.K., Westgren, M., Nixon, D.F., and Michaelsson, J. (2013). Differentiation and functional regulation of human fetal NK cells. J Clin Invest 123, 3889-3901.

Jacobson, L.O., Simmons, E.L., Marks, E.K., and Eldredge, J.H. (1951). Recovery from radiation injury. Science $113,510-511$.

Jaffredo, T., Gautier, R., Eichmann, A., and Dieterlen-Lievre, F. (1998). Intraaortic hemopoietic cells are derived from endothelial cells during ontogeny. Development 125, 4575-4583.

Jaiswal, S., and Ebert, B.L. (2019). Clonal hematopoiesis in human aging and disease. Science 366.

Jaiswal, S., Fontanillas, P., Flannick, J., Manning, A., Grauman, P.V., Mar, B.G., Lindsley, R.C., Mermel, C.H., Burtt, N., Chavez, A., et al. (2014). Age-related clonal hematopoiesis associated with adverse outcomes. N Engl J Med 371, 2488-2498. 
Jaiswal, S., Natarajan, P., Silver, A.J., Gibson, C.J., Bick, A.G., Shvartz, E., McConkey, M., Gupta, N., Gabriel, S., Ardissino, D., et al. (2017). Clonal Hematopoiesis and Risk of Atherosclerotic Cardiovascular Disease. N Engl J Med 377, 111-121.

Johansson, B.M., and Wiles, M.V. (1995). Evidence for involvement of activin A and bone morphogenetic protein 4 in mammalian mesoderm and hematopoietic development. Mol Cell Biol 15, 141-151.

Jordan, H.E. (1917). Aortic Cell Clusters in Vertebrate Embryos. Proc Natl Acad Sci U S A 3, 149-156.

Kabrun, N., Buhring, H.J., Choi, K., Ullrich, A., Risau, W., and Keller, G. (1997). Flk-1 expression defines a population of early embryonic hematopoietic precursors. Development 124, 2039-2048.

Kallianpur, A.R., Jordan, J.E., and Brandt, S.J. (1994). The SCL/TAL-1 gene is expressed in progenitors of both the hematopoietic and vascular systems during embryogenesis. Blood 83 , 1200-1208.

Kamel-Reid, S., and Dick, J.E. (1988). Engraftment of immune-deficient mice with human hematopoietic stem cells. Science 242, 1706-1709.

Karaman, S., Leppänen, V.M., and Alitalo, K. (2018). Vascular endothelial growth factor signaling in development and disease. Development 145.

Katagiri, T., and Watabe, T. (2016). Bone Morphogenetic Proteins. Cold Spring Harb Perspect Biol 8.

Kattman, S.J., Witty, A.D., Gagliardi, M., Dubois, N.C., Niapour, M., Hotta, A., Ellis, J., and Keller, G. (2011). Stage-specific optimization of activin/nodal and BMP signaling promotes cardiac differentiation of mouse and human pluripotent stem cell lines. Cell Stem Cell 8, 228240.

Kaufman, D.S., Hanson, E.T., Lewis, R.L., Auerbach, R., and Thomson, J.A. (2001). Hematopoietic colony-forming cells derived from human embryonic stem cells. Proc Natl Acad Sci U S A 98, 10716-10721.

Keller, G., Paige, C., Gilboa, E., and Wagner, E.F. (1985). Expression of a foreign gene in myeloid and lymphoid cells derived from multipotent haematopoietic precursors. Nature 318 , 149-154.

Keller, G., and Snodgrass, R. (1990). Life span of multipotential hematopoietic stem cells in vivo. J Exp Med 171, 1407-1418.

Kennedy, M., Awong, G., Sturgeon, C.M., Ditadi, A., LaMotte-Mohs, R., Zuniga-Pflucker, J.C., and Keller, G. (2012). T lymphocyte potential marks the emergence of definitive hematopoietic progenitors in human pluripotent stem cell differentiation cultures. Cell Rep 2, 1722-1735. 
Kennedy, M., D'Souza, S.L., Lynch-Kattman, M., Schwantz, S., and Keller, G. (2007). Development of the hemangioblast defines the onset of hematopoiesis in human ES cell differentiation cultures. Blood 109, 2679-2687.

Kennedy, M., Firpo, M., Choi, K., Wall, C., Robertson, S., Kabrun, N., and Keller, G. (1997). A common precursor for primitive erythropoiesis and definitive haematopoiesis. Nature 386, 488493.

Kiel, M.J., Yilmaz, O.H., Iwashita, T., Yilmaz, O.H., Terhorst, C., and Morrison, S.J. (2005). SLAM family receptors distinguish hematopoietic stem and progenitor cells and reveal endothelial niches for stem cells. Cell 121, 1109-1121.

Kingsley, P.D., Malik, J., Fantauzzo, K.A., and Palis, J. (2004). Yolk sac-derived primitive erythroblasts enucleate during mammalian embryogenesis. Blood 104, 19-25.

Kissa, K., and Herbomel, P. (2010). Blood stem cells emerge from aortic endothelium by a novel type of cell transition. Nature 464, 112-115.

Klichinsky, M., Ruella, M., Shestova, O., Lu, X.M., Best, A., Zeeman, M., Schmierer, M., Gabrusiewicz, K., Anderson, N.R., Petty, N.E., et al. (2020). Human chimeric antigen receptor macrophages for cancer immunotherapy. Nature Biotechnology.

Kubo, A., Shinozaki, K., Shannon, J.M., Kouskoff, V., Kennedy, M., Woo, S., Fehling, H.J., and Keller, G. (2004). Development of definitive endoderm from embryonic stem cells in culture. Development 131, 1651-1662.

Kumano, K., Chiba, S., Kunisato, A., Sata, M., Saito, T., Nakagami-Yamaguchi, E., Yamaguchi, T., Masuda, S., Shimizu, K., Takahashi, T., et al. (2003). Notch1 but not Notch2 is essential for generating hematopoietic stem cells from endothelial cells. Immunity 18, 699-711.

Kumaravelu, P., Hook, L., Morrison, A.M., Ure, J., Zhao, S., Zuyev, S., Ansell, J., and Medvinsky, A. (2002). Quantitative developmental anatomy of definitive haematopoietic stem cells/long-term repopulating units (HSC/RUs): role of the aorta-gonad-mesonephros (AGM) region and the yolk sac in colonisation of the mouse embryonic liver. Development 129, 48914899.

Kuziel, W.A., Takashima, A., Bonyhadi, M., Bergstresser, P.R., Allison, J.P., Tigelaar, R.E., and Tucker, P.W. (1987). Regulation of T-cell receptor gamma-chain RNA expression in murine Thy-1+ dendritic epidermal cells. Nature 328, 263-266.

Lacaud, G., Gore, L., Kennedy, M., Kouskoff, V., Kingsley, P., Hogan, C., Carlsson, L., Speck, N., Palis, J., and Keller, G. (2002). Runx1 is essential for hematopoietic commitment at the hemangioblast stage of development in vitro. Blood 100, 458-466.

Lancrin, C., Sroczynska, P., Stephenson, C., Allen, T., Kouskoff, V., and Lacaud, G. (2009). The haemangioblast generates haematopoietic cells through a haemogenic endothelium stage. Nature $457,892-895$. 
Laurenti, E., and Gottgens, B. (2018). From haematopoietic stem cells to complex differentiation landscapes. Nature 553, 418-426.

Lavin, Y., Winter, D., Blecher-Gonen, R., David, E., Keren-Shaul, H., Merad, M., Jung, S., and Amit, I. (2014). Tissue-resident macrophage enhancer landscapes are shaped by the local microenvironment. Cell 159, 1312-1326.

Lavine, K.J., Epelman, S., Uchida, K., Weber, K.J., Nichols, C.G., Schilling, J.D., Ornitz, D.M., Randolph, G.J., and Mann, D.L. (2014). Distinct macrophage lineages contribute to disparate patterns of cardiac recovery and remodeling in the neonatal and adult heart. Proc Natl Acad Sci U S A 111, 16029-16034.

Lee, J.H., Protze, S.I., Laksman, Z., Backx, P.H., and Keller, G.M. (2017). Human Pluripotent Stem Cell-Derived Atrial and Ventricular Cardiomyocytes Develop from Distinct Mesoderm Populations. Cell Stem Cell 21, 179-194.e174.

Lee, Y., Manegold, J.E., Kim, A.D., Pouget, C., Stachura, D.L., Clements, W.K., and Traver, D. (2014). FGF signalling specifies haematopoietic stem cells through its regulation of somitic Notch signalling. Nat Commun 5, 5583.

Lengerke, C., Schmitt, S., Bowman, T.V., Jang, I.H., Maouche-Chretien, L., McKinneyFreeman, S., Davidson, A.J., Hammerschmidt, M., Rentzsch, F., Green, J.B., et al. (2008). BMP and Wnt specify hematopoietic fate by activation of the Cdx-Hox pathway. Cell Stem Cell 2, $72-$ 82.

Lescroart, F., Chabab, S., Lin, X., Rulands, S., Paulissen, C., Rodolosse, A., Auer, H., Achouri, Y., Dubois, C., Bondue, A., et al. (2014). Early lineage restriction in temporally distinct populations of Mesp1 progenitors during mammalian heart development. Nat Cell Biol 16, 829840.

Li, L.B., Ma, C., Awong, G., Kennedy, M., Gornalusse, G., Keller, G., Kaufman, D.S., and Russell, D.W. (2016). Silent IL2RG Gene Editing in Human Pluripotent Stem Cells. Mol Ther $24,582-591$.

Li, W., Batliwalla, F., and Rothstein, T.L. (2013). Human B-1 cells are not preplasmablasts: analysis of microarray data and other issues. Blood 122, 3691-3693.

Li, Z., Lan, Y., He, W., Chen, D., Wang, J., Zhou, F., Wang, Y., Sun, H., Chen, X., Xu, C., et al. (2012). Mouse embryonic head as a site for hematopoietic stem cell development. Cell Stem Cell $11,663-675$.

Li, Z., Liu, S., Xu, J., Zhang, X., Han, D., Liu, J., Xia, M., Yi, L., Shen, Q., Xu, S., et al. (2018). Adult Connective Tissue-Resident Mast Cells Originate from Late Erythro-Myeloid Progenitors. Immunity 49, 640-653.e645.

Liu, E., Marin, D., Banerjee, P., Macapinlac, H.A., Thompson, P., Basar, R., Nassif Kerbauy, L., Overman, B., Thall, P., Kaplan, M., et al. (2020). Use of CAR-Transduced Natural Killer Cells in CD19-Positive Lymphoid Tumors. N Engl J Med 382, 545-553. 
Liu, P., Wakamiya, M., Shea, M.J., Albrecht, U., Behringer, R.R., and Bradley, A. (1999).

Requirement for Wnt3 in vertebrate axis formation. Nat Genet 22, 361-365.

Lorenz, E., Uphoff, D., Reid, T.R., and Shelton, E. (1951). Modification of irradiation injury in mice and guinea pigs by bone marrow injections. J Natl Cancer Inst 12, 197-201.

Lugus, J.J., Park, C., Ma, Y.D., and Choi, K. (2009). Both primitive and definitive blood cells are derived from Flk-1+ mesoderm. Blood 113, 563-566.

Luis, T.C., Luc, S., Mizukami, T., Boukarabila, H., Thongjuea, S., Woll, P.S., Azzoni, E., Giustacchini, A., Lutteropp, M., Bouriez-Jones, T., et al. (2016). Initial seeding of the embryonic thymus by immune-restricted lympho-myeloid progenitors. Nat Immunol 17, 1424-1435.

Lux, C.T., Yoshimoto, M., McGrath, K., Conway, S.J., Palis, J., and Yoder, M.C. (2008). All primitive and definitive hematopoietic progenitor cells emerging before E10 in the mouse embryo are products of the yolk sac. Blood 111,3435-3438.

Maclean, G.A., Menne, T.F., Guo, G., Sanchez, D.J., Park, I.H., Daley, G.Q., and Orkin, S.H. (2012). Altered hematopoiesis in trisomy 21 as revealed through in vitro differentiation of isogenic human pluripotent cells. Proc Natl Acad Sci U S A 109, 17567-17572.

Maguire, J.A., Gagne, A.L., Jobaliya, C.D., Gandre-Babbe, S., Gadue, P., and French, D.L. (2016). Generation of human control iPS cell line CHOPWT10 from healthy adult peripheral blood mononuclear cells. Stem Cell Res 16, 338-341.

Mancuso, R., Van Den Daele, J., Fattorelli, N., Wolfs, L., Balusu, S., Burton, O., Liston, A., Sierksma, A., Fourne, Y., Poovathingal, S., et al. (2019). Stem-cell-derived human microglia transplanted in mouse brain to study human disease. Nat Neurosci 22, 2111-2116.

Martyn, I., Kanno, T.Y., Ruzo, A., Siggia, E.D., and Brivanlou, A.H. (2018). Self-organization of a human organizer by combined Wnt and Nodal signalling. Nature 558, 132-135.

Mass, E., Jacome-Galarza, C.E., Blank, T., Lazarov, T., Durham, B.H., Ozkaya, N., Pastore, A., Schwabenland, M., Chung, Y.R., Rosenblum, M.K., et al. (2017). A somatic mutation in erythromyeloid progenitors causes neurodegenerative disease. Nature 549, 389-393.

Matsuoka, S., Tsuji, K., Hisakawa, H., Xu, M., Ebihara, Y., Ishii, T., Sugiyama, D., Manabe, A., Tanaka, R., Ikeda, Y., et al. (2001). Generation of definitive hematopoietic stem cells from murine early yolk sac and paraaortic splanchnopleures by aorta-gonad-mesonephros regionderived stromal cells. Blood 98, 6-12.

Maude, S.L., Laetsch, T.W., Buechner, J., Rives, S., Boyer, M., Bittencourt, H., Bader, P., Verneris, M.R., Stefanski, H.E., Myers, G.D., et al. (2018). Tisagenlecleucel in Children and Young Adults with B-Cell Lymphoblastic Leukemia. N Engl J Med 378, 439-448.

McGrath, K.E., Frame, J.M., Fegan, K.H., Bowen, J.R., Conway, S.J., Catherman, S.C., Kingsley, P.D., Koniski, A.D., and Palis, J. (2015). Distinct Sources of Hematopoietic Progenitors Emerge before HSCs and Provide Functional Blood Cells in the Mammalian Embryo. Cell Rep 11, 1892-1904. 
McGrath, K.E., Frame, J.M., Fromm, G.J., Koniski, A.D., Kingsley, P.D., Little, J., Bulger, M., and Palis, J. (2011). A transient definitive erythroid lineage with unique regulation of the betaglobin locus in the mammalian embryo. Blood 117, 4600-4608.

McGrath, K.E., Koniski, A.D., Malik, J., and Palis, J. (2003). Circulation is established in a stepwise pattern in the mammalian embryo. Blood 101, 1669-1676.

McMahon, A.P., and Moon, R.T. (1989). Ectopic expression of the proto-oncogene int-1 in Xenopus embryos leads to duplication of the embryonic axis. Cell 58, 1075-1084.

McVay, L.D., and Carding, S.R. (1996). Extrathymic origin of human gamma delta T cells during fetal development. J Immunol 157, 2873-2882.

Medvinsky, A., and Dzierzak, E. (1996). Definitive hematopoiesis is autonomously initiated by the AGM region. Cell 86, 897-906.

Mellentin, J.D., Smith, S.D., and Cleary, M.L. (1989). lyl-1, a novel gene altered by chromosomal translocation in T cell leukemia, codes for a protein with a helix-loop-helix DNA binding motif. Cell 58, 77-83.

Michaelsson, J., Mold, J.E., McCune, J.M., and Nixon, D.F. (2006). Regulation of T cell responses in the developing human fetus. J Immunol 176, 5741-5748.

Migliaccio, G., Migliaccio, A.R., Petti, S., Mavilio, F., Russo, G., Lazzaro, D., Testa, U., Marinucci, M., and Peschle, C. (1986). Human embryonic hemopoiesis. Kinetics of progenitors and precursors underlying the yolk sac----liver transition. J Clin Invest 78, 51-60.

Mikkola, H.K., Fujiwara, Y., Schlaeger, T.M., Traver, D., and Orkin, S.H. (2003a). Expression of CD41 marks the initiation of definitive hematopoiesis in the mouse embryo. Blood 101, 508516.

Mikkola, H.K., Klintman, J., Yang, H., Hock, H., Schlaeger, T.M., Fujiwara, Y., and Orkin, S.H. (2003b). Haematopoietic stem cells retain long-term repopulating activity and multipotency in the absence of stem-cell leukaemia SCL/tal-1 gene. Nature 421, 547-551.

Miller, J., Horner, A., Stacy, T., Lowrey, C., Lian, J.B., Stein, G., Nuckolls, G.H., and Speck, N.A. (2002). The core-binding factor beta subunit is required for bone formation and hematopoietic maturation. Nat Genet 32, 645-649.

Mohtashami, M., Shah, D.K., Kianizad, K., Awong, G., and Zúñiga-Pflücker, J.C. (2013). Induction of T-cell development by Delta-like 4-expressing fibroblasts. Int Immunol 25, 601611.

Mold, J.E., Venkatasubrahmanyam, S., Burt, T.D., Michaëlsson, J., Rivera, J.M., Galkina, S.A., Weinberg, K., Stoddart, C.A., and McCune, J.M. (2010). Fetal and adult hematopoietic stem cells give rise to distinct $\mathrm{T}$ cell lineages in humans. Science 330, 1695-1699.

Mombaerts, P., Iacomini, J., Johnson, R.S., Herrup, K., Tonegawa, S., and Papaioannou, V.E. (1992). RAG-1-deficient mice have no mature B and T lymphocytes. Cell 68, 869-877. 
Montecino-Rodriguez, E., Leathers, H., and Dorshkind, K. (2006). Identification of a B-1 B cellspecified progenitor. Nat Immunol 7, 293-301.

Moore, M.A., and Owen, J.J. (1967). Experimental studies on the development of the thymus. J Exp Med 126, 715-726.

Moroni, F., Dwyer, B.J., Graham, C., Pass, C., Bailey, L., Ritchie, L., Mitchell, D., Glover, A., Laurie, A., Doig, S., et al. (2019). Safety profile of autologous macrophage therapy for liver cirrhosis. Nature Medicine 25, 1560-1565.

Mouthon, M.A., Bernard, O., Mitjavila, M.T., Romeo, P.H., Vainchenker, W., and MathieuMahul, D. (1993). Expression of tal-1 and GATA-binding proteins during human hematopoiesis. Blood 81, 647-655.

Mucenski, M.L., McLain, K., Kier, A.B., Swerdlow, S.H., Schreiner, C.M., Miller, T.A., Pietryga, D.W., Scott, W.J., Jr., and Potter, S.S. (1991). A functional c-myb gene is required for normal murine fetal hepatic hematopoiesis. Cell 65, 677-689.

Muffat, J., Li, Y., Yuan, B., Mitalipova, M., Omer, A., Corcoran, S., Bakiasi, G., Tsai, L.H., Aubourg, P., Ransohoff, R.M., et al. (2016). Efficient derivation of microglia-like cells from human pluripotent stem cells. Nat Med 22, 1358-1367.

Mukouyama, Y., Chiba, N., Mucenski, M.L., Satake, M., Miyajima, A., Hara, T., and Watanabe, T. (1999). Hematopoietic cells in cultures of the murine embryonic aorta-gonad-mesonephros region are induced by c-Myb. Curr Biol 9, 833-836.

Muller, A.M., Medvinsky, A., Strouboulis, J., Grosveld, F., and Dzierzak, E. (1994).

Development of hematopoietic stem cell activity in the mouse embryo. Immunity 1, 291-301.

Müller, A.M., Medvinsky, A., Strouboulis, J., Grosveld, F., and Dzierzak, E. (1994).

Development of hematopoietic stem cell activity in the mouse embryo. Immunity 1, 291-301.

Murray, P.D.F. (1932). The Development in vitro of the Blood of the Early Chick Embryo.

Proceedings of the Royal Society of London Series B, Containing Papers of a Biological

Character 111, 497-521.

Naito, M., Yamamura, F., Nishikawa, S., and Takahashi, K. (1989). Development, differentiation, and maturation of fetal mouse yolk sac macrophages in cultures. J Leukoc Biol 46, 1-10.

NCT03841110 (2019). FT500 as Monotherapy and in Combination With Immune Checkpoint Inhibitors in Subjects With Advanced Solid Tumors. Sponsor: Fate Therapeutics. Investigator: Wayne Chu. https://clinicaltrials.gov/ct2/show/NCT03841110.

NCT04363346 (2020). Study of FT516 for the Treatment of COVID-19 in Hospitalized Patients With Hypoxia. Sponsor: Fate Therapeutics. Investigator: Joshua Rhein. https://clinicaltrials.gov/ct2/show/NCT04363346. 
Ng, E.S., Azzola, L., Bruveris, F.F., Calvanese, V., Phipson, B., Vlahos, K., Hirst, C., Jokubaitis, V.J., Yu, Q.C., Maksimovic, J., et al. (2016). Differentiation of human embryonic stem cells to $\mathrm{HOXA}(+)$ hemogenic vasculature that resembles the aorta-gonad-mesonephros. Nat Biotechnol $34,1168-1179$.

Niederreither, K., McCaffery, P., Drager, U.C., Chambon, P., and Dolle, P. (1997). Restricted expression and retinoic acid-induced downregulation of the retinaldehyde dehydrogenase type 2 (RALDH-2) gene during mouse development. Mech Dev 62, 67-78.

Niederreither, K., Subbarayan, V., Dolle, P., and Chambon, P. (1999). Embryonic retinoic acid synthesis is essential for early mouse post-implantation development. Nat Genet 21, 444-448.

Nishikawa, S.I., Nishikawa, S., Hirashima, M., Matsuyoshi, N., and Kodama, H. (1998a). Progressive lineage analysis by cell sorting and culture identifies FLK1+VE-cadherin+ cells at a diverging point of endothelial and hemopoietic lineages. Development 125, 1747-1757.

Nishikawa, S.I., Nishikawa, S., Kawamoto, H., Yoshida, H., Kizumoto, M., Kataoka, H., and Katsura, Y. (1998b). In vitro generation of lymphohematopoietic cells from endothelial cells purified from murine embryos. Immunity 8, 761-769.

North, T., Gu, T.L., Stacy, T., Wang, Q., Howard, L., Binder, M., Marin-Padilla, M., and Speck, N.A. (1999). Cbfa2 is required for the formation of intra-aortic hematopoietic clusters.

Development 126, 2563-2575.

North, T.E., de Bruijn, M.F., Stacy, T., Talebian, L., Lind, E., Robin, C., Binder, M., Dzierzak, E., and Speck, N.A. (2002). Runx1 expression marks long-term repopulating hematopoietic stem cells in the midgestation mouse embryo. Immunity 16, 661-672.

Nostro, M.C., Cheng, X., Keller, G.M., and Gadue, P. (2008). Wnt, activin, and BMP signaling regulate distinct stages in the developmental pathway from embryonic stem cells to blood. Cell Stem Cell 2, 60-71.

Notta, F., Doulatov, S., Laurenti, E., Poeppl, A., Jurisica, I., and Dick, J.E. (2011). Isolation of single human hematopoietic stem cells capable of long-term multilineage engraftment. Science $333,218-221$.

Oelgeschlager, M., Janknecht, R., Krieg, J., Schreek, S., and Luscher, B. (1996). Interaction of the co-activator CBP with Myb proteins: effects on Myb-specific transactivation and on the cooperativity with NF-M. Embo j 15, 2771-2780.

Ogilvy, S., Elefanty, A.G., Visvader, J., Bath, M.L., Harris, A.W., and Adams, J.M. (1998). Transcriptional regulation of vav, a gene expressed throughout the hematopoietic compartment. Blood 91, 419-430.

Ogilvy, S., Metcalf, D., Gibson, L., Bath, M.L., Harris, A.W., and Adams, J.M. (1999). Promoter elements of vav drive transgene expression in vivo throughout the hematopoietic compartment. Blood 94, 1855-1863. 
Oka, C., Nakano, T., Wakeham, A., de la Pompa, J.L., Mori, C., Sakai, T., Okazaki, S., Kawaichi, M., Shiota, K., Mak, T.W., et al. (1995). Disruption of the mouse RBP-J kappa gene results in early embryonic death. Development 121, 3291-3301.

Okuda, T., van Deursen, J., Hiebert, S.W., Grosveld, G., and Downing, J.R. (1996). AML1, the target of multiple chromosomal translocations in human leukemia, is essential for normal fetal liver hematopoiesis. Cell 84, 321-330.

Osawa, M., Hanada, K., Hamada, H., and Nakauchi, H. (1996). Long-term lymphohematopoietic reconstitution by a single CD34-low/negative hematopoietic stem cell. Science 273, 242-245.

Ottersbach, K., and Dzierzak, E. (2005). The murine placenta contains hematopoietic stem cells within the vascular labyrinth region. Dev Cell 8, 377-387.

Palis, J., Robertson, S., Kennedy, M., Wall, C., and Keller, G. (1999). Development of erythroid and myeloid progenitors in the yolk sac and embryo proper of the mouse. Development 126 , 5073-5084.

Pandya, H., Shen, M.J., Ichikawa, D.M., Sedlock, A.B., Choi, Y., Johnson, K.R., Kim, G., Brown, M.A., Elkahloun, A.G., Maric, D., et al. (2017). Differentiation of human and murine induced pluripotent stem cells to microglia-like cells. Nat Neurosci 20, 753-759.

Park, C., Afrikanova, I., Chung, Y.S., Zhang, W.J., Arentson, E., Fong Gh, G., Rosendahl, A., and Choi, K. (2004). A hierarchical order of factors in the generation of FLK1- and SCLexpressing hematopoietic and endothelial progenitors from embryonic stem cells. Development $131,2749-2762$.

Park, J.E., Botting, R.A., Dominguez Conde, C., Popescu, D.M., Lavaert, M., Kunz, D.J., Goh, I., Stephenson, E., Ragazzini, R., Tuck, E., et al. (2020). A cell atlas of human thymic development defines $\mathrm{T}$ cell repertoire formation. Science 367.

Park, J.H., Riviere, I., Gonen, M., Wang, X., Senechal, B., Curran, K.J., Sauter, C., Wang, Y., Santomasso, B., Mead, E., et al. (2018). Long-Term Follow-up of CD19 CAR Therapy in Acute Lymphoblastic Leukemia. N Engl J Med 378, 449-459.

Pauklin, S., and Vallier, L. (2015). Activin/Nodal signalling in stem cells. Development 142, 607-619.

Pearson, S., Sroczynska, P., Lacaud, G., and Kouskoff, V. (2008). The stepwise specification of embryonic stem cells to hematopoietic fate is driven by sequential exposure to Bmp4, activin A, bFGF and VEGF. Development 135, 1525-1535.

Pei, W., Feyerabend, T.B., Rössler, J., Wang, X., Postrach, D., Busch, K., Rode, I., Klapproth, K., Dietlein, N., Quedenau, C., et al. (2017). Polylox barcoding reveals haematopoietic stem cell fates realized in vivo. Nature 548, 456-460.

Peng, H., Jiang, X., Chen, Y., Sojka, D.K., Wei, H., Gao, X., Sun, R., Yokoyama, W.M., and Tian, Z. (2013). Liver-resident NK cells confer adaptive immunity in skin-contact inflammation. J Clin Invest 123, 1444-1456. 
Perez-Andres, M., Grosserichter-Wagener, C., Teodosio, C., van Dongen, J.J., Orfao, A., and van Zelm, M.C. (2011). The nature of circulating CD27+CD43+ B cells. J Exp Med 208, 25652566.

Perlingeiro, R.C. (2007). Endoglin is required for hemangioblast and early hematopoietic development. Development 134, 3041-3048.

Peschle, C., Mavilio, F., Care, A., Migliaccio, G., Migliaccio, A.R., Salvo, G., Samoggia, P., Petti, S., Guerriero, R., Marinucci, M., et al. (1985). Haemoglobin switching in human embryos: asynchrony of zeta----alpha and epsilon----gamma-globin switches in primitive and definite erythropoietic lineage. Nature $313,235-238$.

Peschle, C., Migliaccio, A.R., Migliaccio, G., Petrini, M., Calandrini, M., Russo, G., Mastroberardino, G., Presta, M., Gianni, A.M., Comi, P., et al. (1984). Embryonic----Fetal Hb switch in humans: studies on erythroid bursts generated by embryonic progenitors from yolk sac and liver. Proc Natl Acad Sci U S A 81, 2416-2420.

Phillips, J.H., Hori, T., Nagler, A., Bhat, N., Spits, H., and Lanier, L.L. (1992). Ontogeny of human natural killer (NK) cells: fetal NK cells mediate cytolytic function and express cytoplasmic CD3 epsilon, delta proteins. J Exp Med 175, 1055-1066.

Pick, M., Azzola, L., Mossman, A., Stanley, E.G., and Elefanty, A.G. (2007). Differentiation of human embryonic stem cells in serum-free medium reveals distinct roles for bone morphogenetic protein 4, vascular endothelial growth factor, stem cell factor, and fibroblast growth factor 2 in hematopoiesis. Stem Cells 25, 2206-2214.

Porcher, C., Swat, W., Rockwell, K., Fujiwara, Y., Alt, F.W., and Orkin, S.H. (1996). The T cell leukemia oncoprotein SCL/tal-1 is essential for development of all hematopoietic lineages. Cell $86,47-57$.

Pouget, C., Peterkin, T., Simoes, F.C., Lee, Y., Traver, D., and Patient, R. (2014). FGF signalling restricts haematopoietic stem cell specification via modulation of the BMP pathway. Nat Commun 5, 5588.

Puck, J.M., Deschenes, S.M., Porter, J.C., Dutra, A.S., Brown, C.J., Willard, H.F., and Henthorn, P.S. (1993). The interleukin-2 receptor gamma chain maps to Xq13.1 and is mutated in X-linked severe combined immunodeficiency, SCIDX1. Hum Mol Genet 2, 1099-1104.

Raffin, C., Vo, L.T., and Bluestone, J.A. (2020). T(reg) cell-based therapies: challenges and perspectives. Nat Rev Immunol 20, 158-172.

Ran, F.A., Hsu, P.D., Wright, J., Agarwala, V., Scott, D.A., and Zhang, F. (2013). Genome engineering using the CRISPR-Cas9 system. Nat Protoc 8, 2281-2308.

Rathinam, C., Poueymirou, W.T., Rojas, J., Murphy, A.J., Valenzuela, D.M., Yancopoulos, G.D., Rongvaux, A., Eynon, E.E., Manz, M.G., and Flavell, R.A. (2011). Efficient differentiation and function of human macrophages in humanized CSF-1 mice. Blood 118, 3119-3128. 
Reaume, A.G., Conlon, R.A., Zirngibl, R., Yamaguchi, T.P., and Rossant, J. (1992). Expression analysis of a Notch homologue in the mouse embryo. Dev Biol 154, 377-387.

Rhinn, M., and Dolle, P. (2012). Retinoic acid signalling during development. Development 139, 843-858.

Robb, L., Elwood, N.J., Elefanty, A.G., Kontgen, F., Li, R., Barnett, L.D., and Begley, C.G. (1996). The scl gene product is required for the generation of all hematopoietic lineages in the adult mouse. Embo j 15, 4123-4129.

Robb, L., Lyons, I., Li, R., Hartley, L., Kontgen, F., Harvey, R.P., Metcalf, D., and Begley, C.G. (1995). Absence of yolk sac hematopoiesis from mice with a targeted disruption of the scl gene. Proc Natl Acad Sci U S A 92, 7075-7079.

Robin, C., Bollerot, K., Mendes, S., Haak, E., Crisan, M., Cerisoli, F., Lauw, I., Kaimakis, P., Jorna, R., Vermeulen, M., et al. (2009). Human placenta is a potent hematopoietic niche containing hematopoietic stem and progenitor cells throughout development. Cell Stem Cell 5, 385-395.

Rönn, R.E., Guibentif, C., Moraghebi, R., Chaves, P., Saxena, S., Garcia, B., and Woods, N.B. (2015). Retinoic acid regulates hematopoietic development from human pluripotent stem cells. Stem Cell Reports 4, 269-281.

Rybtsov, S., Batsivari, A., Bilotkach, K., Paruzina, D., Senserrich, J., Nerushev, O., and Medvinsky, A. (2014). Tracing the origin of the HSC hierarchy reveals an SCF-dependent, IL-3independent CD43(-) embryonic precursor. Stem Cell Reports 3, 489-501.

Rydeen, A.B., and Waxman, J.S. (2014). Cyp26 enzymes are required to balance the cardiac and vascular lineages within the anterior lateral plate mesoderm. Development 141, 1638-1648.

Sabin, F.R. (1920). Studies on the Origin of Blood-vessels and of Red Blood-corpuscles as Seen in the Living Blastoderm of Chicks During the Second Day of Incubation (Carnegie Institution of Washington).

Samokhvalov, I.M., Samokhvalova, N.I., and Nishikawa, S. (2007). Cell tracing shows the contribution of the yolk sac to adult haematopoiesis. Nature 446, 1056-1061.

Sanchez, M.J., Holmes, A., Miles, C., and Dzierzak, E. (1996). Characterization of the first definitive hematopoietic stem cells in the AGM and liver of the mouse embryo. Immunity 5 , 513-525.

Scala, S., Basso-Ricci, L., Dionisio, F., Pellin, D., Giannelli, S., Salerio, F.A., Leonardelli, L., Cicalese, M.P., Ferrua, F., Aiuti, A., et al. (2018). Dynamics of genetically engineered hematopoietic stem and progenitor cells after autologous transplantation in humans. Nat Med 24, 1683-1690.

Schmitt, T.M., and Zuniga-Pflucker, J.C. (2002). Induction of T cell development from hematopoietic progenitor cells by delta-like-1 in vitro. Immunity 17, 749-756. 
Schmitt, T.M., and Zuniga-Pflucker, J.C. (2006). T-cell development, doing it in a dish. Immunol Rev 209, 95-102.

Schröder, A.R., Shinn, P., Chen, H., Berry, C., Ecker, J.R., and Bushman, F. (2002). HIV-1 integration in the human genome favors active genes and local hotspots. Cell 110, 521-529.

Schulz, C., Gomez Perdiguero, E., Chorro, L., Szabo-Rogers, H., Cagnard, N., Kierdorf, K., Prinz, M., Wu, B., Jacobsen, S.E., Pollard, J.W., et al. (2012). A lineage of myeloid cells independent of Myb and hematopoietic stem cells. Science 336, 86-90.

Schwarz, K., Gauss, G.H., Ludwig, L., Pannicke, U., Li, Z., Lindner, D., Friedrich, W., Seger, R.A., Hansen-Hagge, T.E., Desiderio, S., et al. (1996). RAG mutations in human B cell-negative SCID. Science 274, 97-99.

Seandel, M., Butler, J.M., Kobayashi, H., Hooper, A.T., White, I.A., Zhang, F., Vertes, E.L., Kobayashi, M., Zhang, Y., Shmelkov, S.V., et al. (2008). Generation of a functional and durable vascular niche by the adenoviral E4ORF1 gene. Proc Natl Acad Sci U S A 105, 19288-19293.

Shalaby, F., Ho, J., Stanford, W.L., Fischer, K.D., Schuh, A.C., Schwartz, L., Bernstein, A., and Rossant, J. (1997). A requirement for Flk1 in primitive and definitive hematopoiesis and vasculogenesis. Cell 89, 981-990.

Shalaby, F., Rossant, J., Yamaguchi, T.P., Gertsenstein, M., Wu, X.F., Breitman, M.L., and Schuh, A.C. (1995). Failure of blood-island formation and vasculogenesis in Flk-1-deficient mice. Nature 376, 62-66.

Shen, M.M. (2007). Nodal signaling: developmental roles and regulation. Development 134, 1023-1034.

Shivdasani, R.A., Mayer, E.L., and Orkin, S.H. (1995). Absence of blood formation in mice lacking the T-cell leukaemia oncoprotein tal-1/SCL. Nature 373, 432-434.

Shurtleff, S.A., Buijs, A., Behm, F.G., Rubnitz, J.E., Raimondi, S.C., Hancock, M.L., Chan, G.C., Pui, C.H., Grosveld, G., and Downing, J.R. (1995). TEL/AML1 fusion resulting from a cryptic $\mathrm{t}(12 ; 21)$ is the most common genetic lesion in pediatric ALL and defines a subgroup of patients with an excellent prognosis. Leukemia 9, 1985-1989.

Shwachman, H., Diamond, L.K., Oski, F.A., and Khaw, K.T. (1964). The syndrome of pancreatic insufficiency and bone marrow dysfunction. J Pediatr 65, 645-663.

Siminovitch, L., McCulloch, E.A., and Till, J.E. (1963). THE DISTRIBUTION OF COLONYFORMING CELLS AMONG SPLEEN COLONIES. J Cell Comp Physiol 62, 327-336.

Sojka, D.K., Plougastel-Douglas, B., Yang, L., Pak-Wittel, M.A., Artyomov, M.N., Ivanova, Y., Zhong, C., Chase, J.M., Rothman, P.B., Yu, J., et al. (2014). Tissue-resident natural killer (NK) cells are cell lineages distinct from thymic and conventional splenic NK cells. eLife 3, e01659. 
Souilhol, C., Lendinez, J.G., Rybtsov, S., Murphy, F., Wilson, H., Hills, D., Batsivari, A., Binagui-Casas, A., McGarvey, A.C., MacDonald, H.R., et al. (2016). Developing HSCs become Notch independent by the end of maturation in the AGM region. Blood 128, 1567-1577.

Stefanska, M., Batta, K., Patel, R., Florkowska, M., Kouskoff, V., and Lacaud, G. (2017). Primitive erythrocytes are generated from hemogenic endothelial cells. Sci Rep 7, 6401.

Steinhart, Z., and Angers, S. (2018). Wnt signaling in development and tissue homeostasis. Development 145.

Stingl, G., Koning, F., Yamada, H., Yokoyama, W.M., Tschachler, E., Bluestone, J.A., Steiner, G., Samelson, L.E., Lew, A.M., Coligan, J.E., et al. (1987). Thy-1+ dendritic epidermal cells express T3 antigen and the T-cell receptor gamma chain. Proc Natl Acad Sci U S A 84, 45864590.

Storms, R.W., Trujillo, A.P., Springer, J.B., Shah, L., Colvin, O.M., Ludeman, S.M., and Smith, C. (1999). Isolation of primitive human hematopoietic progenitors on the basis of aldehyde dehydrogenase activity. Proc Natl Acad Sci U S A 96, 9118-9123.

Sturgeon, C.M., Ditadi, A., Awong, G., Kennedy, M., and Keller, G. (2014). Wnt signaling controls the specification of definitive and primitive hematopoiesis from human pluripotent stem cells. Nat Biotechnol 32, 554-561.

Sugimura, R., Jha, D.K., Han, A., Soria-Valles, C., da Rocha, E.L., Lu, Y.F., Goettel, J.A., Serrao, E., Rowe, R.G., Malleshaiah, M., et al. (2017). Haematopoietic stem and progenitor cells from human pluripotent stem cells. Nature 545, 432-438.

Sun, J., Ramos, A., Chapman, B., Johnnidis, J.B., Le, L., Ho, Y.J., Klein, A., Hofmann, O., and Camargo, F.D. (2014). Clonal dynamics of native haematopoiesis. Nature 514, 322-327.

Suzuki, N., Yamazaki, S., Yamaguchi, T., Okabe, M., Masaki, H., Takaki, S., Otsu, M., and Nakauchi, H. (2013). Generation of engraftable hematopoietic stem cells from induced pluripotent stem cells by way of teratoma formation. Mol Ther 21, 1424-1431.

Swiatek, P.J., Lindsell, C.E., del Amo, F.F., Weinmaster, G., and Gridley, T. (1994). Notch1 is essential for postimplantation development in mice. Genes Dev 8, 707-719.

Swiers, G., Baumann, C., O'Rourke, J., Giannoulatou, E., Taylor, S., Joshi, A., Moignard, V., Pina, C., Bee, T., Kokkaliaris, K.D., et al. (2013). Early dynamic fate changes in haemogenic endothelium characterized at the single-cell level. Nat Commun 4, 2924.

Takahashi, K., Yamamura, F., and Naito, M. (1989). Differentiation, maturation, and proliferation of macrophages in the mouse yolk sac: a light-microscopic, enzyme-cytochemical, immunohistochemical, and ultrastructural study. J Leukoc Biol 45, 87-96.

Takata, K., Kozaki, T., Lee, C.Z.W., Thion, M.S., Otsuka, M., Lim, S., Utami, K.H., Fidan, K., Park, D.S., Malleret, B., et al. (2017). Induced-Pluripotent-Stem-Cell-Derived Primitive Macrophages Provide a Platform for Modeling Tissue-Resident Macrophage Differentiation and Function. Immunity 47, 183-198.e186. 
Tang, Y., Harrington, A., Yang, X., Friesel, R.E., and Liaw, L. (2010). The contribution of the Tie2+ lineage to primitive and definitive hematopoietic cells. Genesis 48, 563-567.

Tavian, M., Hallais, M.F., and Peault, B. (1999). Emergence of intraembryonic hematopoietic precursors in the pre-liver human embryo. Development 126, 793-803.

Themeli, M., Kloss, C.C., Ciriello, G., Fedorov, V.D., Perna, F., Gonen, M., and Sadelain, M. (2013). Generation of tumor-targeted human T lymphocytes from induced pluripotent stem cells for cancer therapy. Nat Biotechnol 31, 928-933.

Thisse, B., Wright, C.V., and Thisse, C. (2000). Activin- and Nodal-related factors control antero-posterior patterning of the zebrafish embryo. Nature 403, 425-428.

Thomas, E.D., Lochte, H.L., Jr., Lu, W.C., and Ferrebee, J.W. (1957). Intravenous infusion of bone marrow in patients receiving radiation and chemotherapy. N Engl J Med 257, 491-496.

Thomson, J.A., Itskovitz-Eldor, J., Shapiro, S.S., Waknitz, M.A., Swiergiel, J.J., Marshall, V.S., and Jones, J.M. (1998). Embryonic stem cell lines derived from human blastocysts. Science 282, 1145-1147.

Till, J.E., and McCulloch, E.A. (1961). A direct measurement of the radiation sensitivity of normal mouse bone marrow cells. Radiat Res 14, 213-222.

Tober, J., Koniski, A., McGrath, K.E., Vemishetti, R., Emerson, R., de Mesy-Bentley, K.K., Waugh, R., and Palis, J. (2007). The megakaryocyte lineage originates from hemangioblast precursors and is an integral component both of primitive and of definitive hematopoiesis. Blood $109,1433-1441$.

Tober, J., McGrath, K.E., and Palis, J. (2008). Primitive erythropoiesis and megakaryopoiesis in the yolk sac are independent of c-myb. Blood 111, 2636-2639.

Tripodo, C., Iannitto, E., Florena, A.M., Pucillo, C.E., Piccaluga, P.P., Franco, V., and Pileri, S.A. (2009). Gamma-delta T-cell lymphomas. Nat Rev Clin Oncol 6, 707-717.

Tulpule, A., Kelley, J.M., Lensch, M.W., McPherson, J., Park, I.H., Hartung, O., Nakamura, T., Schlaeger, T.M., Shimamura, A., and Daley, G.Q. (2013). Pluripotent stem cell models of Shwachman-Diamond syndrome reveal a common mechanism for pancreatic and hematopoietic dysfunction. Cell Stem Cell 12, 727-736.

Turner, N., and Grose, R. (2010). Fibroblast growth factor signalling: from development to cancer. Nat Rev Cancer 10, 116-129.

Uenishi, G.I., Jung, H.S., Kumar, A., Park, M.A., Hadland, B.K., McLeod, E., Raymond, M., Moskvin, O., Zimmerman, C.E., Theisen, D.J., et al. (2018). NOTCH signaling specifies arterialtype definitive hemogenic endothelium from human pluripotent stem cells. Nat Commun 9 , 1828. 
Vale, A.M., Kearney, J.F., Nobrega, A., and Schroeder, H.W. (2015). Chapter 7 - Development and Function of B Cell Subsets. In Molecular Biology of B Cells (Second Edition), F.W. Alt, T. Honjo, A. Radbruch, and M. Reth, eds. (London: Academic Press), pp. 99-119.

Vallier, L., Alexander, M., and Pedersen, R.A. (2005). Activin/Nodal and FGF pathways cooperate to maintain pluripotency of human embryonic stem cells. J Cell Sci 118, 4495-4509.

Van Handel, B., Montel-Hagen, A., Sasidharan, R., Nakano, H., Ferrari, R., Boogerd, C.J., Schredelseker, J., Wang, Y., Hunter, S., Org, T., et al. (2012). Scl represses cardiomyogenesis in prospective hemogenic endothelium and endocardium. Cell 150, 590-605.

Vanhee, S., De Mulder, K., Van Caeneghem, Y., Verstichel, G., Van Roy, N., Menten, B., Velghe, I., Philippé, J., De Bleser, D., Lambrecht, B.N., et al. (2015). In vitro human embryonic stem cell hematopoiesis mimics MYB-independent yolk sac hematopoiesis. Haematologica 100, 157-166.

Varol, C., Mildner, A., and Jung, S. (2015). Macrophages: development and tissue specialization. Annu Rev Immunol 33, 643-675.

Villa, N., Walker, L., Lindsell, C.E., Gasson, J., Iruela-Arispe, M.L., and Weinmaster, G. (2001). Vascular expression of Notch pathway receptors and ligands is restricted to arterial vessels. Mech Dev 108, 161-164.

Vodyanik, M.A., Bork, J.A., Thomson, J.A., and Slukvin, II (2005). Human embryonic stem cell-derived CD34+ cells: efficient production in the coculture with OP9 stromal cells and analysis of lymphohematopoietic potential. Blood 105, 617-626.

Vodyanik, M.A., Yu, J., Zhang, X., Tian, S., Stewart, R., Thomson, J.A., and Slukvin, II (2010). A mesoderm-derived precursor for mesenchymal stem and endothelial cells. Cell Stem Cell 7, 718-729.

Wang, C., Tang, X., Sun, X., Miao, Z., Lv, Y., Yang, Y., Zhang, H., Zhang, P., Liu, Y., Du, L., et al. (2012). TGF $\beta$ inhibition enhances the generation of hematopoietic progenitors from human ES cell-derived hemogenic endothelial cells using a stepwise strategy. Cell Res 22, 194-207.

Wang, Q., Stacy, T., Binder, M., Marin-Padilla, M., Sharpe, A.H., and Speck, N.A. (1996a). Disruption of the Cbfa2 gene causes necrosis and hemorrhaging in the central nervous system and blocks definitive hematopoiesis. Proc Natl Acad Sci U S A 93, 3444-3449.

Wang, Q., Stacy, T., Miller, J.D., Lewis, A.F., Gu, T.L., Huang, X., Bushweller, J.H., Bories, J.C., Alt, F.W., Ryan, G., et al. (1996b). The CBFbeta subunit is essential for CBFalpha2 (AML1) function in vivo. Cell 87, 697-708.

Wang, R.N., Green, J., Wang, Z., Deng, Y., Qiao, M., Peabody, M., Zhang, Q., Ye, J., Yan, Z., Denduluri, S., et al. (2014). Bone Morphogenetic Protein (BMP) signaling in development and human diseases. Genes Dis 1, 87-105.

Weinmaster, G., Roberts, V.J., and Lemke, G. (1992). Notch2: a second mammalian Notch gene. Development 116, 931-941. 
Wilkinson, D.G., Bhatt, S., and Herrmann, B.G. (1990). Expression pattern of the mouse T gene and its role in mesoderm formation. Nature 343, 657-659.

Williams, D.A., Lemischka, I.R., Nathan, D.G., and Mulligan, R.C. (1984). Introduction of new genetic material into pluripotent haematopoietic stem cells of the mouse. Nature 310,476-480.

Winnier, G., Blessing, M., Labosky, P.A., and Hogan, B.L. (1995). Bone morphogenetic protein4 is required for mesoderm formation and patterning in the mouse. Genes Dev 9, 2105-2116.

Wong, P.M., Chung, S.W., Chui, D.H., and Eaves, C.J. (1986a). Properties of the earliest clonogenic hemopoietic precursors to appear in the developing murine yolk sac. Proc Natl Acad Sci U S A 83, 3851-3854.

Wong, P.M., Chung, S.W., Reicheld, S.M., and Chui, D.H. (1986b). Hemoglobin switching during murine embryonic development: evidence for two populations of embryonic erythropoietic progenitor cells. Blood 67, 716-721.

Wu, A.M., Till, J.E., Siminovitch, L., and McCulloch, E.A. (1968). Cytological evidence for a relationship between normal hemotopoietic colony-forming cells and cells of the lymphoid system. J Exp Med 127, 455-464.

Xu, M.J., Matsuoka, S., Yang, F.C., Ebihara, Y., Manabe, A., Tanaka, R., Eguchi, M., Asano, S., Nakahata, T., and Tsuji, K. (2001). Evidence for the presence of murine primitive megakaryocytopoiesis in the early yolk sac. Blood 97, 2016-2022.

Yamaguchi, T.P., Dumont, D.J., Conlon, R.A., Breitman, M.L., and Rossant, J. (1993). flk-1, an flt-related receptor tyrosine kinase is an early marker for endothelial cell precursors. Development 118, 489-498.

Yamaguchi, T.P., Harpal, K., Henkemeyer, M., and Rossant, J. (1994). fgfr-1 is required for embryonic growth and mesodermal patterning during mouse gastrulation. Genes Dev 8, 30323044.

Yoder, M.C., and Hiatt, K. (1997). Engraftment of embryonic hematopoietic cells in conditioned newborn recipients. Blood 89, 2176-2183.

Yoder, M.C., Hiatt, K., Dutt, P., Mukherjee, P., Bodine, D.M., and Orlic, D. (1997a). Characterization of definitive lymphohematopoietic stem cells in the day 9 murine yolk sac. Immunity 7, 335-344.

Yoder, M.C., Hiatt, K., and Mukherjee, P. (1997b). In vivo repopulating hematopoietic stem cells are present in the murine yolk sac at day 9.0 postcoitus. Proc Natl Acad Sci U S A 94, 6776-6780.

Yoshimoto, M., Montecino-Rodriguez, E., Ferkowicz, M.J., Porayette, P., Shelley, W.C., Conway, S.J., Dorshkind, K., and Yoder, M.C. (2011). Embryonic day 9 yolk sac and intraembryonic hemogenic endothelium independently generate a B-1 and marginal zone progenitor lacking B-2 potential. Proc Natl Acad Sci U S A 108, 1468-1473. 
Yoshimoto, M., Porayette, P., Glosson, N.L., Conway, S.J., Carlesso, N., Cardoso, A.A., Kaplan, M.H., and Yoder, M.C. (2012). Autonomous murine T-cell progenitor production in the extraembryonic yolk sac before HSC emergence. Blood 119, 5706-5714.

Yu, C., Liu, Y., Miao, Z., Yin, M., Lu, W., Lv, Y., Ding, M., and Deng, H. (2010). Retinoic acid enhances the generation of hematopoietic progenitors from human embryonic stem cell-derived hemato-vascular precursors. Blood 116, 4786-4794.

Zambidis, E.T., Park, T.S., Yu, W., Tam, A., Levine, M., Yuan, X., Pryzhkova, M., and Peault, B. (2008). Expression of angiotensin-converting enzyme (CD143) identifies and regulates primitive hemangioblasts derived from human pluripotent stem cells. Blood 112, 3601-3614.

Zeng, Y., Liu, C., Gong, Y., Bai, Z., Hou, S., He, J., Bian, Z., Li, Z., Ni, Y., Yan, J., et al. (2019). Single-Cell RNA Sequencing Resolves Spatiotemporal Development of Pre-thymic Lymphoid Progenitors and Thymus Organogenesis in Human Embryos. Immunity 51, 930948.e936.

Zhang, H., and Bradley, A. (1996). Mice deficient for BMP2 are nonviable and have defects in amnion/chorion and cardiac development. Development 122, 2977-2986.

Zhang, P., Li, J., Tan, Z., Wang, C., Liu, T., Chen, L., Yong, J., Jiang, W., Sun, X., Du, L., et al. (2008). Short-term BMP-4 treatment initiates mesoderm induction in human embryonic stem cells. Blood 111, 1933-1941.

Zhou, X., Sasaki, H., Lowe, L., Hogan, B.L., and Kuehn, M.R. (1993). Nodal is a novel TGFbeta-like gene expressed in the mouse node during gastrulation. Nature 361, 543-547. 